\title{
Global, regional, and national incidence and mortality for HIV, tuberculosis, and malaria during 1990-2013: a systematic analysis for the Global Burden of Disease Study 2013
}

\author{
A full list of authors and affiliations appears at the end of the article.
}

\section{Summary}

Background-The Millennium Declaration in 2000 brought special global attention to HIV, tuberculosis, and malaria through the formulation of Millennium Development Goal (MDG) 6 . The Global Burden of Disease 2013 study provides a consistent and comprehensive approach to disease estimation for between 1990 and 2013, and an opportunity to assess whether accelerated progress has occurred since the Millennium Declaration.

Methods-To estimate incidence and mortality for HIV, we used the UNAIDS Spectrum model appropriately modified based on a systematic review of available studies of mortality with and without antiretroviral therapy (ART). For concentrated epidemics, we calibrated Spectrum models to fit vital registration data corrected for misclassification of HIV deaths. In generalised epidemics, we minimised a loss function to select epidemic curves most consistent with prevalence data and demographic data for all-cause mortality. We analysed counterfactual scenarios for HIV to assess years of life saved through prevention of mother-to-child transmission (PMTCT) and ART. For tuberculosis, we analysed vital registration and verbal autopsy data to estimate mortality using cause of death ensemble modelling. We analysed data for corrected casenotifications, expert opinions on the case-detection rate, prevalence surveys, and estimated causespecific mortality using Bayesian meta-regression to generate consistent trends in all parameters.

Correspondence to: Christopher J L Murray.

Contributors: CJLM, ADL, and TV prepared the first draft. CJLM, KFO, CG, SSL, TMW, DAR, EAD, NG, RMB, JCB, HCD, LD, JAS, DEP, TDF, BKP, EKJ, MSC, ADL, and TV finalised the draft based on comments from other authors and reviewer feedback. CJLM, SSL, and TV had the idea for the study and provided overall guidance. CJLM, KFO, CG, SSL, TMW, DAR, EAD, NG, HW, MN, DD, KRH, KF, DEP, TDF, ADF, and TV did all modeling. CJLM, KFO, CG, SSL, TMW, DAR, EAD, NG, RMB, HW, HCD, DD, DEP, ADF, and TV did the statistical analysis of model results. All other authors provided data, developed models, reviewed results, initiated modeling infrastructure, and reviewed the paper.

$\dagger$ Authors listed alphabetically

末Joint senior authors

Declaration of interests: $\mathrm{CC}$ has received consultancy, lecture fees and honoraria from AMGEN, GSK, Alliance for Better Bone Health, MSD, Eli Lilly, Pfizer, Novartis, Servier, Merck, Medtronic and Roche. LD has been funded by and acted as a consultant to UNAIDS, UNODC, and WHO. BD Gessner works for AMP which receives grant specific support from Crucell, GSK, Novartis, Pfizer, and SanofiPasteur and unrestricted support from SanofiPasteur all for work on vaccines and vaccine preventable diseases. Related to the current work, AMP receives grant support from GSK for work on issues related to malaria vaccines. KBG received the NHMRC-Gustav Nossal scholarship sponsored by CSL in 2012. This award is peer-reviewed through the standard NHMRC peerreview process; CSL played no part in selection of the awardee. PJ is supported by a career development fellowship from the Wellcome Trust, Public Health Foundation of India and a Consortium of United Kingdom Universities. Walter Mendoza is program analyst at the UNFPA country office in Peru, which not necessarily endorses the study. JAS has received research grants from Takeda and Savient and consultant fees from Savient, Takeda, Regeneron and Allergan. JAS is a member of the executive of OMERACT, an organization that develops outcome measures in rheumatology and receives arms-length funding from 36 companies; a member of the American College of Rheumatology's Guidelines Subcommittee of the Quality of Care Committee; and a member of the Veterans Affairs Rheumatology Field Advisory Committee. 
We analysed malaria mortality and incidence using an updated cause of death database, a systematic analysis of verbal autopsy validation studies for malaria, and recent studies (2010-13) of incidence, drug resistance, and coverage of insecticide-treated bednets.

Findings-Globally in 2013, there were 1.8 million new HIV infections (95\% uncertainty interval 1.7 million to 2.1 million), 29.2 million prevalent HIV cases (28.1 to 31.7 ), and 1.3 million HIV deaths (1.3 to 1.5). At the peak of the epidemic in 2005, HIV caused 1.7 million deaths (1.6 million to 1.9 million). Concentrated epidemics in Latin America and eastern Europe are substantially smaller than previously estimated. Through interventions including PMTCT and ART, 19.1 million life-years (16.6 million to 21.5 million) have been saved, $70.3 \%$ (65.4 to $76 \cdot 1$ ) in developing countries. From 2000 to 2011, the ratio of development assistance for health for HIV to years of life saved through intervention was US $\$ 4498$ in developing countries. Including in HIV-positive individuals, all-form tuberculosis incidence was 7.5 million (7.4 million to 7.7 million), prevalence was 11.9 million (11.6 million to 12.2 million), and number of deaths was 1.4 million (1.3 million to 1.5 million) in 2013. In the same year and in only individuals who were HIV-negative, all-form tuberculosis incidence was 7.1 million (6.9 million to 7.3 million), prevalence was 11.2 million (10.8 million to 11.6 million), and number of deaths was 1.3 million (1.2 million to 1.4 million). Annualised rates of change (ARC) for incidence, prevalence, and death became negative after 2000. Tuberculosis in HIV-negative individuals disproportionately occurs in men and boys (versus women and girls); $64.0 \%$ of cases (63.6 to 64.3 ) and $64.7 \%$ of deaths $(60 \cdot 8$ to $70 \cdot 3)$. Globally, malaria cases and deaths grew rapidly from 1990 reaching a peak of 232 million cases (143 million to 387 million) in 2003 and 1.2 million deaths ( 1.1 million to 1.4 million) in 2004. Since 2004, child deaths from malaria in sub-Saharan Africa have decreased by $31.5 \%$ (15.7 to $44 \cdot 1)$. Outside of Africa, malaria mortality has been steadily decreasing since 1990.

Interpretation-Our estimates of the number of people living with HIV are 18.7\% smaller than UNAIDS's estimates in 2012. The number of people living with malaria is larger than estimated by WHO. The number of people living with HIV, tuberculosis, or malaria have all decreased since 2000. At the global level, upward trends for malaria and HIV deaths have been reversed and declines in tuberculosis deaths have accelerated. 101 countries (74 of which are developing) still have increasing HIV incidence. Substantial progress since the Millennium Declaration is an encouraging sign of the effect of global action.

Funding-Bill \& Melinda Gates Foundation.

\section{Introduction}

The Millennium Declaration in 2000 brought special global attention to HIV, tuberculosis, and malaria through the formulation of Millennium Development Goal 6 (MDG 6). The high priority status of these three diseases in the development community was confirmed through the creation of the Global Fund to Fight AIDS, Tuberculosis and Malaria in 2002. Bilateral initiatives such as the President's Emergency Plan for AIDS Relief and the President's Malaria Initiative also added substantial new resources. From 2000 to 2011, multilaterals, bilaterals, foundations, and non-governmental organisations have invested US $\$ 51.6$ billion for HIV, $\$ 11.3$ billion for malaria, and $\$ 8.3$ billion for tuberculosis (price in 2011 US dollars) in development assistance for health (DAH). ${ }^{1}$ Substantial benefits of these 
investments have been documented in several studies. ${ }^{2-9}$ In the lead up to the MDG deadline of 2015 and amid the global debate on development goals post-2015, important questions have been raised about the advantages and disadvantages of maintaining focus on these three diseases. ${ }^{5,10-18}$ The rise of the importance of non-communicable diseases in some regions of the developing world ${ }^{19-26}$ have led to calls for goals that cover a broader range of diseases. ${ }^{10,15,17,19,20,24,25,27,28}$ At the same time, ambitious goals of zero tuberculosis incidence and deaths and zero HIV incidence and deaths have been formulated by some groups; ${ }^{29-34}$ the Secretary-General of the UN had already established a goal of zero malaria deaths by $2015 .{ }^{35}$ Understanding the distribution and trends of these three diseases and how they have been affected by the MDG era is an important input to this wider debate. ${ }^{12,36}$

Because of their prominence, there are major UN efforts on an annual basis to track the epidemiology of these three diseases. UNAIDS now produces country estimates of HIV incidence, prevalence, and death every year. ${ }^{37}$ Over many years, they have developed a sophisticated modelling approach to track the epidemic - their primary input in generalised epidemics is annual antenatal clinic sero-surveillance data and periodic household surveys that include blood testing. ${ }^{38,39}$ The annual Global Tuberculosis Report from the World Health Organization (WHO) provides estimates of incidence and deaths from tuberculosis by country. Crucial inputs to the assessment of incidence are case notifications and national expert opinion on the case-detection rate, and separate modelling of cause of death data from vital registration systems and verbal autopsy studies. For the World Malaria Report, WHO uses a complicated strategy to estimate incidence and mortality that varies by region and age group. For child malaria deaths in sub-Saharan Africa, the main inputs are verbal autopsy studies and estimated malaria risk. Estimates are adjusted post-hoc for coverage of insecticide-treated bednets (ITNs). Outside of sub-Saharan Africa and for low-transmission countries in Africa reported case numbers are combined with an assumed case-fatality rate. These three efforts have provided important insights into the geographical distribution and likely trends in the diseases.

Despite these efforts, extraordinary uncertainty exists at the country-level in the burden of all three. The burdens of HIV and malaria are concentrated in sub-Saharan Africa; countries that, other than South Africa, have very poor vital registration and incomplete notification systems. Tuberculosis is concentrated in Asia and southern Africa where a few more countries have better data systems but there are still huge gaps in information. Modelling strategies for tracking the diseases have evolved to be necessarily complex in view of the incomplete and often conflicting nature of the data. For HIV and malaria, UN modelling efforts explicitly use information about intervention delivery and assumed benefits of intervention. The distinction between data for disease outcomes and data for intervention coverage driving the results of these efforts is blurred. In the more complex modelling strategies, the compounded effect of uncertainty about different parameters can be hard to characterise. Efforts to model the three diseases are largely independent of each other-the exception is recent coordinated efforts to understand the intersection of tuberculosis and HIV. $^{40}$

The Global Burden of Disease 2010 Study provided a comprehensive update on levels and trends of a large number of diseases, injuries, and risk factors for 187 countries from 1990 to 
2010. ${ }^{41-48}$ The Global Burden of Disease collaboration is now generating annual updates, the first of which is the Global Burden of Disease, Injuries, and Risk Factors Study 2013 (GBD 2013). The GBD 2013 provides an opportunity to examine the evidence on the levels and trends in the three MDG 6 diseases within the comprehensive and coherent framework of the GBD. Compared with GBD 2010, we have given special emphasis in the GBD 2013 to incorporate new data, to more rigorously identify and incorporate further key sources of uncertainty, and to incorporate adjustments for the biases that are present in different data sources. A crucial aspect of the GBD effort is to quantify time trends; comparing trends from 1990 to 2000 and from 2000 to 2013 provides an opportunity to see if there has been accelerated progress since the Millennium Declaration. The GBD 2013 supersedes all previously published GBD results.

\section{Methods}

\section{Overview}

The overall conceptual and analytical framework for the GBD is described elsewhere. ${ }^{41-48}$ Major refinements of the analytical approach for different diseases and risk factors are explored in other papers. ${ }^{49-51}$ We summarise here the methods used for the analysis of the three diseases, emphasising refinements since the GBD 2010. All refinements in methods have been applied to the full 1990-2013 time series to ensure comparability of results. Metadata for input sources used in the GBD 2013 will be available in the Global Health Data Exchange on publication of the full GBD 2013 results.

\section{HIV}

For the GBD 2010, we primarily used estimates of prevalence and mortality developed by UNAIDS. The main modification was the requirement that the sum of cause-specific mortality from each cause in a country, age, sex, and year group equalled the estimate of allcause mortality for that country, age, and sex group generated through the analysis of demographic sources. ${ }^{43}$ Modifications of HIV deaths through this internal consistency process did not lead to revisions of incidence or prevalence for HIV in a particular age-sexcountry-year. For the GBD 2013, we have sought to develop a set of estimates of incidence, prevalence, and mortality from HIV that are internally consistent with each other and also meet the GBD requirement that the sum of each cause of death equals all-cause mortality. Internally consistent means the incidence, prevalence, and death figures are mathematically possible given that prevalence is a function of past incidence, remission, and death rates for any age cohort.

Modified Spectrum Model-UNAIDS uses two key analytical components in their epidemiological estimation. The Estimation and Projections Package (EPP) is used to estimate incidence trajectories that are consistent with prevalence surveys and other prevalence measurements such as antenatal clinic serosurveillance. ${ }^{52}$ Spectrum is a compartmental HIV progression model used to generate age-specific incidence, prevalence, and death rates from the EPP incidence curves and assumptions about intervention scale-up and local variation in epidemiology. ${ }^{53}$ We have recoded Spectrum in Python, an open source higher level language that can easily be run on a parallelised computational cluster, 
following the exact structure of Spectrum to facilitate faster computation required for the uncertainty analysis and consistency analysis with all-cause mortality. We have also made four important modifications to the assumptions.

First, we have altered the Spectrum assumptions about mortality without antiretroviral therapy (ART). Following UNAIDS assumptions, mortality is modelled as shown in figure 1. The death and progression rates between CD4 categories vary by age according to four age-groups, 15-24 years, 25-34 years, 35-44 years, and 45 years or older. UNAIDS estimates a single set of progression and death rates by first fitting a Weibull distribution to data from three east African seroconverter cohorts from the ALPHA network and one miners cohort from South Africa, ${ }^{54}$ and then selecting a set of progression and death rates in Excel Solver that minimises the sum of the squared differences between the predicted and Weibull survival probabilities. ${ }^{55,56}$ Uncertainty in their estimates for this component was approximated by assuming a coefficient of variation of 0.05 for each mortality rate. To better characterise uncertainty in the progression and death rates, we systematically reviewed the literature on mortality without ART. We searched terms related to pre-ART or ART-naive survival since seroconversion-exact search terms are in the appendix (p 132). After screening, we identified 13 cohort studies that included the cohorts used by UNAIDS from which we extracted survival at each 1-year point after infection. We modelled the logit of the conditional probability of death between years in these studies using the following formula:

$$
\operatorname{logit}\left(m_{i j k}\right)=\beta_{0}+\sum_{i=1}^{4} \beta_{1 i} a_{i}+\sum_{j=1}^{12} \beta_{2 j} t_{j}+u_{k}+\varepsilon_{i j k}
$$

In the formula, $m$ is conditional probability of death from year $t_{j}$ to $t_{\mathrm{j}+1}, a_{i}$ is an indicator variable for age group at seroconversion (15-24 years, 25-34 years, 35-44 years, and 45 years or older), $t_{\mathrm{j}}$ is an indicator variable of year since seroconversion, and $u_{\mathrm{K}}$ is a studylevel random effect. By sampling the variance-covariance matrix of the regression coefficients and the study level random effect, we generated 1000 survival curves for each age group that capture the systematic variation in survival across the available studies (figure 2, appendix p 18). Across all age groups, median survival ranges from 3.6 years to 29.5 years. For each of the 1000 survival curves, we use the UNAIDS optimisation framework to find a set of progression and death rates that minimises the sum of the squared errors for the fit to the survival curve. For the death rates generated from the optimisation, the coefficient of variation across the set of 1000 is $0.44-1.00$ depending on the age-group, which is substantially higher than the UNAIDS assumption of 0.05.

Second, for mortality on ART, UNAIDS used data from five regional cohorts from the IeDEA network to directly estimate death rates by age, sex, and CD4 count, which have been used as the default for all countries in a region. 57,58 Through their country consultation process some of these defaults have been modified. For example, Myanmar assumes a constant mortality rate by initial CD4 group, without any variation by age, sex, or time on treatment. To better characterise real variation in the death rates on ART across programmes, we searched the published literature. Using the terms "HIV", "mortality", and 
"antiretroviral therapy" we identified 4996 titles. Screening the abstracts and papers yielded 102 total papers for extraction (appendix p 133). These included mortality and loss to follow-up data for 80 cohorts, age hazard ratios for 40 cohorts, and sex hazard ratios for 86 cohorts. We corrected reported probabilities of death for loss to follow-up using an update of the approach developed by Verguet and colleagues. ${ }^{59}$ Verguet and colleagues used tracing and follow-up studies to empirically estimate the relation between death in loss to follow-up and the rate of loss to follow-up. We used DisMod-MR 2.0 to do a meta-regression of the data for on-ART mortality by initial CD4 count separately for high-income countries, GBD developing countries outside of sub-Saharan Africa, and sub-Saharan Africa (appendix p 7). We meta-analysed region-specific age hazard ratios using DisMod-MR 2.0, and regionspecific sex hazard ratios using the Stata command metan. The age and sex hazard ratios were applied to the CD4-specific mortality rates, accounting for the distribution of ages and sexes in the mortality data. We used 1000 draws from the posterior distribution for each age, sex, and CD4 category for conditional probabilities of death for 0-6 months, 7-12 months, and 13-24 months after initiation of ART as inputs to Spectrum. Table 1 shows HIVspecific mortality rates for people aged 25-34 years on ART in sub-Saharan Africa (see appendix pp 136-38 for HIV-specific mortality for other age groups and regions).

Third, to better capture variation in the age-pattern of incidence, we used the UNAIDS distributions of the relative incidence by age prepared for UNAIDS based on selected cohort studies. To capture the possibility that there is greater variation across countries in the age incidence pattern than in these studies, we increased the uncertainty ranges by an arbitrary $50 \%$.

Fourth, for all other input parameters including the number of individuals receiving ART, prevention of mother-to-child transmission (PMTCT), or co-trimoxazole prophylaxis, we randomly sampled a uniform distribution from 0.9 to 1.1 and used the draw to adjust each parameter. For the sex ratio of incidence, we sampled a wider but arbitrary range from 0.8 to $1 \cdot 2$, because the demographic data in many generalised epidemics indicate that there is less of a difference between the sexes than seen in the population prevalence surveys.

Generalised epidemics-UNAIDS identified 41 countries as generalised epidemics; this distinction is important because for these epidemics the primary sources of information about prevalence come from antenatal clinic serosurveillance and household surveys. In addition to these 41 countries, we have included in this category Senegal, Niger, and India because of the availability of population-based surveys. Prevalence data from countries with generalised epidemics has been analysed by UNAIDS using EPP to generate 1000 samples of incidence curves for people aged 15-49 years consistent with the prevalence data. For each of these 1000 incidence curves, we randomly sampled the parameter distributions for all input parameters ten times to generate 10000 epidemic curves of incidence, prevalence, and death by age and sex. The selection of 10000 iterations was based on testing that it would ensure stable uncertainty intervals. Some of these 10000 death curves exceed in one or more age-sex-year groups the estimate of all-cause mortality based on demographic sources. Because the demographic estimates of all-cause mortality are based on substantial empirical data, these HIV epidemic curves are unlikely to represent reality. These mismatches occur more often in southern Africa. We identified the 250 modified Spectrum 
curves and all-cause mortality curves that are most consistent with each other. We define a loss function using the following formula:

$$
e_{r}=\sum_{t} \sum_{a} \sum_{s} \max \left(0, m_{r, t, a, s}^{H I V}-0 \cdot 8^{*} m_{r, t, a, s}^{\text {all-cause }}\right)
$$

For run $r$ of a given country, excess mortality, $e$, is equal to the sum of all non-zero differences between HIV mortality, $m^{H I V}$, and 0.8 times a randomly selected all-cause mortality draw, $m^{\text {all-cause }}$, across all year-age-sex combinations ( $t, a$, and $s$, respectively). We compared the Spectrum estimates to 0.8 times the all-cause estimates because this is the highest recorded fraction of deaths in any age-group in any country's cause of death data due to HIV. We randomly paired each of the 10000 modified spectrum outputs with one of the 1000 all-cause mortality curves generated from the demographic analysis. The 250 pairs that minimised the loss function were selected. When more than 250 have a loss function equal to zero, we randomly chose among this set. We resampled the 250 draws to $1000 ; 250$ are used with resampling for computational convenience. The appendix (pp 18-20) shows mortality at ages 15-59 years from the full set of 10000 modified spectrum models and the subset that is selected through the matching process for Uganda, South Africa, and Ghana. Demographic data matching selects in South Africa epidemic curves that are at the low end of the distribution with longer median survival; in Uganda this effect is slightly less pronounced and in Ghana pre-matching and post-matching were identical.

Concentrated epidemics with vital registration data-UNAIDS estimates for concentrated epidemics depend critically on two inputs: first, the assessment of prevalence of HIV in high-risk groups (people who inject drugs, men who have sex with men, and female sex workers) for which there is much information in many countries; ${ }^{60-72}$ and second, assumptions on the percentage of the population in high-risk groups. Although there is guidance on measurement, ${ }^{73}$ real data in most countries are limited. In many countries, UNAIDS estimates are based on local opinion. Resulting assumptions have been highly variable across countries. For example, Uruguay defines $4.5 \%$ of its population as men who have sex with men while neighbouring Argentina defines only $1 \%$ of its population as men who have sex with men. An alternative source in many countries is cause of death data from national vital registration systems. To track the epidemic using cause of death data can require up to three important adjustments. First, in some middle-income countries, vital registration is incomplete. Wang and colleagues have used death distribution methods ${ }^{43}$ to assess completeness in all countries with vital registration; we have used this information to correct upwards incomplete registration. Second, a key aspect of the GBD is to redistribute deaths that are assigned to immediate or intermediate causes of death rather than underlying causes of death (garbage codes). ${ }^{74}$ In addition to garbage codes, because HIV was not included in the International Classification of Diseases (ICD)-9 until 1986 and not implemented in many countries until later, deaths were often assigned to other codes such as graft versus host disease or Kaposi's sarcoma. Third, in some places, because of stigma or misdiagnosis, HIV deaths can be assigned to other underlying causes of death such as tuberculosis, endocrine disorders, meningitis, or encephalitis. Birnbaum and colleagues developed a method to identify these misclassified deaths. ${ }^{75}$ We applied this method to all 
countries. Misclassification of HIV deaths in Birnbaum and colleagues' method is based on fulfilling three criteria. First, the temporal trend for a cause should coincide with the HIV epidemic. Second, the pattern of the relative death rate by age should shift towards the ages of 15-49 years during the epidemic years. Third, the temporal and age-pattern shifts cannot be explained by other known epidemiological trends. Applying these methods, we transfer deaths from 47 causes in 52 countries. Figure 3 shows the number of HIV deaths directly coded to HIV, the number of deaths re-assigned to HIV from garbage code redistribution, and the number of deaths from the application of the HIV misclassification method for Thailand and Russia. The height of the bar is the final number of deaths in each age group.

For countries with corrected vital registration, we imputed missing years of data to generate a complete time series for HIV from the estimated start year of the epidemic using spatialtemporal Gaussian Process Regression (ST-GPR). ${ }^{43,76,77}$ ST-GPR using a linear mean function and a Matern covariance has been widely used for time-series estimation in global health descriptive studies such as for tobacco prevalence, obesity prevalence, or child and adult mortality. To generate an internally consistent set of incidence, prevalence, and death curves with uncertainty, we used the observed HIV death numbers to calibrate the modified Spectrum models. First, we started with a modified Spectrum model constructed based on the analysis of high-risk groups - where no high-risk group analysis was available we used a regional default model. Second, for each of the 1000 draws from this model, we modified the incidence at time $t$ by the ratio of observed deaths to modified spectrum deaths at time $t$ $+\lambda$, where $\lambda$ is the lag between incidence and death. We drew from a distribution of lags of 10-15 years to generate 6000 different adjusted incidence curves. For incidence for the last $\lambda$ time periods, we drew a random weight between 0 and 1 from a uniform distribution and used it to calculate a weighted average of adjusted incidence in year $t+\lambda$ and unadjusted incidence multiplied by the deaths ratio in year $t+\lambda$. Using these modified incidence curves, we reran the modified Spectrum generating 6000 possible epidemic curves. As a final step, for each of these 6000 we computed the mean squared error of predicted deaths compared with observed deaths. The 1000 curves with the lowest mean squared error were selected as the final set for analysis. Figure 4 shows the results of this process for Panama and the comparison with the UNAIDS high-risk group analysis; the corrected vital registration data suggest a much smaller epidemic with different timing.

\section{Concentrated epidemics with high-risk group analysis and insufficient or no} cause of death data-There were 17 countries with concentrated epidemics where we had no or insufficient vital registration or verbal autopsy data to inform our cause of death analysis. For these countries we ran modified Spectrum to output 1000 draws of incidence, randomly selecting 1000 time series of the death ratios generated in the process described above, and multiplying each draw of incidence by the selected set of ratios. We selected incidence adjustments only for countries with a cumulative number of HIV deaths greater than 5000 to avoid exaggerated stochastic variation in the ratios. We then derived estimates of mortality by running the adjusted incidence curves back through Spectrum. By using random draws across these countries, the average correction and uncertainty in this correction is propagated into the corrections for these countries with little or no data. 


\section{Concentrated epidemics with no high-risk group analysis and no cause of} death data-For 13 countries (Andorra, United Arab Emirates, Iraq, Federated States of Micronesia, Libya, Marshall Islands, State of Palestine, Solomon Islands, Timor-Leste, Vanuatu, Samoa, Tonga, and North Korea) no analysis of high-risk groups has been undertaken and no cause of death data are available. For these countries, we picked regional or neighbouring countries to approximate the death rate. We used these approximate death rates to fit a Spectrum model. In all these cases, the number of estimated deaths was less than 250 per year.

Comparisons to prevalence survey data-As a form of empirical validation, we compared our final estimated prevalence with national population-based surveys collected through the Demographic and Health Surveys, AIDS Indicator Surveys, the 2005-2006 Indian National Family Health Survey, and the 2012 South African National HIV Prevalence, Incidence, and Behaviour Survey. ${ }^{78-80}$ In total, we extracted data from 46 surveys in 35 countries between 2001 and 2012. These surveys had response rates for HIV testing ranging from $63 \%$ in male respondents in Malawi in 2004 to $98 \%$ for both sexes in Rwanda in 2011; median response rate was $85 \%$. These comparisons are made for adults aged 15-49 by sex and 5-year age groups. We tested for significant differences in means $(\mathrm{p}<0.05)$ for each estimate and compared the distribution of survey estimates to GBD and UNAIDS via ordinary least squares (OLS) regression with robust standard errors to account for heteroscedasticity.

HIV intervention counterfactual scenario-Spectrum uses as inputs the numbers reported by governments of individuals receiving PMTCT, co-trimoxazole, and child and adult ART. To help understand the role of interventions including ART, PMTCT, and cotrimoxazole prophylaxis, we have rerun the final 1000 modified Spectrum models for each country using a no intervention counterfactual scenario. We turn all HIV-related interventions to zero including ART, PMTCT, and co-trimoxazole prophylaxis for all years. We compared the number of deaths and person-years lived each year from the base case to this counterfactual to assess the changes due to intervention. Using published results on DAH for HIV, we computed the ratio of DAH to years of life saved.

\section{Tuberculosis}

For the GBD 2010, we estimated tuberculosis mortality and then estimated population incidence through mixed effects regression as a function of tuberculosis mortality, casenotifications, and an indicator variable for health system access used as a proxy for completeness of registration. For GBD 2013, we have shifted to using all available data for different outcomes and simultaneously estimating incidence, remission, excess mortality, prevalence, and cause-specific mortality using the GBD Bayesian meta-regression environment, DisMod-MR 2.0 (appendix p 11). There are four potential sources of information to estimate national levels and trends for tuberculosis in a country: annual case notifications, expert judgment on the case-detection rate, prevalence surveys, and cause of death data. Additionally, to facilitate convergence of the meta-regression, estimated excess mortality and remission rates have been used. The approach is predicated on the principle that incidence, prevalence, and mortality might be measured imperfectly and that a statistical 
triangulation of all the sources for a country will provide a more robust assessment. Our meta-regression analysis was done for all forms of tuberculosis. As a final step we estimated incidence, prevalence, and death in individuals who are HIV-positive and those who are HIV-negative. We explain in more detail the preparation of each of these sources.

Adjusted case notifications and incidence-Case definitions for tuberculosis since 1995 have been standardised by WHO and widely applied. Countries have varied however in the completeness of reporting for younger age-groups and some countries have reported only pulmonary smear-positive cases for selected years. We use the age and sex-specific notifications in our analysis and impute the missing age-groups for three forms of tuberculosis notifications (pulmonary smear-positive, pulmonary smear-negative, or extrapulmonary) in two steps. First, for each country-sex category with missing age-groups in some years, we imputed the missing values by regressing the log of the case notification rate on dummy variables for 5-year age-groups and random effects on year using all the data for a country over the interval 1990-2013.

Second, we estimated the relation between all forms of tuberculosis and smear-positive tuberculosis and the relation between all forms and bacteriologically positive tuberculosis. Using country-years with complete notifications (all three forms), we used a compositional analysis model to simultaneously estimate the fraction of cases due to all three forms as a function of dummy variables for 5-year age-groups and sex and the smear-positive tuberculosis rate. This regression was used to impute missing values for pulmonary smearnegative and extra-pulmonary cases. Because of substantial variation in the diagnostic rates for extrapulmonary tuberculosis and the potential for misclassification, we used the predicted values for extrapulmonary cases for all countries from the regression.

At the country-level several smear-unknown and relapsed cases are recorded that are not captured in the age-specific and sex-specific notifications. We used the relation between these forms and pulmonary smear-positive cases in the country-level data to inflate the adjusted age-specific and sex-specific pulmonary smear-positive notifications used in our analysis. Case notifications, however, do not capture all true incidence cases in the population. Case notifications can be incomplete because some cases are not diagnosed and some diagnosed cases are not reported to the national tuberculosis programme. Populationbased incidence studies for tuberculosis based on active surveillance are rare and have not been done at the national level. ${ }^{81}$ In the absence of direct measurement of true incidence, the case detection rate must be approximated. Since 2008, WHO has been consulting with national tuberculosis programme managers in 96 countries to collect expert opinion on the case detection rate including some notion of subjective uncertainty. ${ }^{82}$ For the remaining countries, case-detection estimates are based on the judgment of WHO staff. We divided adjusted case notifications by the estimated case detection rate to generate the incidence inputs used for DisMod-MR 2.0. We expanded the subjective uncertainty intervals reported so they are at a minimum plus or minus $20 \%$ from the estimated values or for values less than $20 \%$ we assume the standard error is half the midpoint estimate.

Tuberculosis prevalence surveys-Prevalence surveys have been periodically undertaken in a few countries such as South Korea and China. WHO standardised the 
protocol and the Global Fund to Fight AIDS, Tuberculosis and Malaria has helped fund 24 surveys in 21 countries between 2002 and 2013 with 12 additional surveys in eight new countries planned. ${ }^{82-84}$ Because the prevalence rates for tuberculosis are often comparatively low (eg, 200 per 100000 population), prevalence surveys need to be large to provide breakdowns by age and sex. On the basis of the literature and country reports, we have identified 27 national and 24 subnational prevalence surveys in 24 countries spanning the time period 1985-2013. We have included in our analysis, surveys reporting on pulmonary smear-positive tuberculosis and bacteriologically positive tuberculosis. We included in the Bayesian meta-regression study level dummy variables for the different measured outcomes with the reference category being bacteriologically positive. We allowed for non-sampling variance for sub-national surveys to be larger which effectively down-weighted their importance for the estimation in a given country. Because mortality and incidence data are for all forms of tuberculosis, we adjusted prevalence surveys to account for extra pulmonary cases using the same factors used in the adjustment of case notification data.

Mortality-We used 2731 country-years of nationally representative vital registration data and 166 site-years of verbal autopsy data to estimate tuberculosis mortality. Vital registration data were adjusted for garbage coding following GBD algorithms ${ }^{74,85}$ and misclassified HIV deaths described above. We modelled deaths by age and sex for tuberculosis using the Cause of Death Ensemble modelling (CODEm) approach. ${ }^{76} \mathrm{CODEm}$ has been extensively used in global cause of death analyses. ${ }^{74}$ Using CODEm, we tested a wide range of potential models and used out-of-sample predictive validity to select the best individual models and the best ensemble of these models. The appendix (pp 152-186) shows details on the application of CODEm to tuberculosis. We ran CODEm separately for male and female individuals. The final ensemble models selected had a root-mean squared error of the log of the age-specific death rate of 0.29 in-sample and 0.63 out-of-sample for males and 0.70 in-sample and 1.05 out-of-sample for females. In the out-of-sample predictive validity testing (cross-validation), the coverage of the $95 \%$ data prediction uncertainty interval was $93 \%$ and $91 \%$ for males and females, respectively.

CODEm results are largely informed by ICD-coded data, which by definition exclude tuberculosis mortality in HIV-positive individuals. The overall Bayesian model, however, is for all forms of tuberculosis in HIV-negative and HIV-positive people because prevalence data rarely distinguish HIV status. We estimated the fraction of HIV deaths due to tuberculosis-HIV and added these to tuberculosis mortality in HIV-negative people. The model for the fraction of tuberculosis-HIV mortality was based on 1022 country-years of data when cause of death data for tuberculosis-HIV and HIV overall were available. We estimated the relation between the logit-transformed fraction of HIV deaths due to tuberculosis-HIV and the log-transformed tuberculosis death rate, a dummy variable for sex, year, and country random effects. We used this regression to predict the fraction of HIV deaths due to tuberculosis-HIV in all countries.

Remission and excess mortality estimates-To help inform the model, we generated a Bayesian prior for remission by examining the ratio of incidence to prevalence in the 
country-years where prevalence surveys have been undertaken. We used a simple regression with random effects to generate priors for countries with surveys and those without. Causespecific mortality estimates inform estimates of prevalence through excess mortality in DisMod-MR 2.0. To provide the model with the range of age-specific and sex-specific excess mortality hazards associated with tuberculosis we analysed historical data where we had both tuberculosis mortality data and incidence data that were believed to be nearly complete. For this analysis, we used the WHO case notifications from 1980 onwards with the supplement of age-sex-specific case notifications back to the 1950s for Australia, Canada, the UK, USA, Japan, and Germany. Case notification data were combined with tuberculosis deaths recorded in the vital registration systems to generate 743 country-year observations from 70 countries that could be used to inform our analysis. We estimated the relation between incidence and mortality for each sex, by regressing the logit-transformed ratio of incidence to mortality against age, lag-distributed income per head, and country random effects. The addition of HIV prevalence off-ART to the regression gave inconsistent coefficients between females and males and was not included in the final model. We estimated the relation between incidence and prevalence as a function of lag-distributed income per head with country random effects. We transformed predicted death to incidence ratios and incidence to prevalence ratios into estimates of excess mortality and remission using the mathematical relations between them (appendix $\mathrm{p} 7$ ).

DisMod-MR 2.0-For each country we included in the DisMod-MR 2.0 estimation the adjusted case notifications, prevalence survey data if available, estimated excess mortality hazard by age and sex, estimated remission, and the tuberculosis-HIV adjusted causespecific mortality estimates from our CODEm model. DisMod-MR 2.0 provides internally consistent estimates for 1990, 1995, 2000, 2005, 2010, and 2013 for 188 countries of incidence, remission, excess mortality, prevalence, and cause-specific mortality using all forms of data or priors in the estimation. Figure 5 shows the internally consistent fit for Kenya in 2013. For intervening years, we interpolated rates.

\section{Estimating tuberculosis incidence, prevalence, and death in individuals who} are HIV-positive-We used tuberculosis all-forms estimates from DisMod-MR 2.0 to estimate incidence and prevalence in HIV-positive people using a relative risk approach. We reviewed the literature using the search terms "incidence", "risk ratio", "HIV", "tuberculosis", and "antiretroviral therapy" and used meta-regression to estimate a relative risk of tuberculosis incidence in HIV-positive individuals in the absence of ARTs based on seven studies ${ }^{86-92}$ of 8.7 (95\% CI 5.9-11.7). Findings from previous studies show that the relative risk of tuberculosis incidence is a function of CD4 count and ART; to parse out the increasing risk ratios of tuberculosis by decreasing $\mathrm{CD} 4$ count and the decreasing risk ratio on ART we used data from the Badri and colleagues' study. ${ }^{93}$ The relative risks we calculated from this analysis were $15 \cdot 7(10 \cdot 6-21 \cdot 1)$ for a CD4 cell count less than $200,10 \cdot 8$ (7.3-14.5) for a cell count of 200-350, 3.2 (2.2-4.3) for a count greater than 350, and $1 \cdot 7$ $(1 \cdot 2-2 \cdot 3)$ for the on-ART category. We computed population-attributable fractions for each category using the outputs of Spectrum above. For prevalence, we assumed that each category of incident tuberculosis cases in HIV-positive individuals has the same duration. Tuberculosis-HIV mortality was estimated as described above. 
Murray and colleagues developed estimates of mortality and incidence for malaria for the GBD 2010. ${ }^{74,94}$ They estimated malaria mortality using vital registration and verbal autopsy data analysed using CODEm. Published community incidence studies were meta-analysed to generate a model of incidence as a function of mortality, age, sex and region. We have modified this method for the GBD 2013 update. Much debate emerged since the publication of that analysis on the validity of verbal autopsy for adult malaria deaths. ${ }^{95-100}$ For the GBD 2013, we undertook a systematic review of the literature on the validity of verbal autopsy for malaria. Our inclusion criteria were validation studies that used physician-certified verbal autopsies, reported both sensitivity and specificity for malaria, and had hospital diagnosis as the gold standard. However, the quality of the gold standards used in these studies was variable, and in some of them malaria cases were not confirmed with a blood smear or did not use a case definition with a threshold of parasitaemia. We identified seven studies. ${ }^{101-107}$ We first tested in a meta-regression if there was any statistically significant difference between studies with and without parasitaemia confirmation and identified none. We metaanalysed these studies to estimate sensitivity and specificity, separately for children and adults. Forest plots for adult and children are shown in figure 6. As a sensitivity analysis, we used this correction but it leads to substantially larger numbers of estimated deaths in adults from malaria (appendix p 21). We have chosen not to correct the data for the main results of this paper because it would adjust deaths in adults upwards which is contrary to expert opinion in the literature.

In view of the fact that we have not applied the sensitivity and specificity corrections, we have instead modified the redistribution of garbage codes such as fever of unknown origin or ill-defined deaths, so that we do not redistribute garbage codes to malaria in adults. We have also updated all the times-series covariates tested in the models: rainfall, health-system access, antimalarial drug resistance weighted by drug use, ITN coverage, indoor residual spraying coverage, income per head, and educational attainment. We have also included in the model the 2010 P falciparum parasite rate (PfPR) map from the Malaria Atlas Project. ${ }^{108}$ A coherent analysis of PfPR overtime is underway but was not available for this analysis (see the appendix pp 223-40 for details on the CODEm model analysis). As in the Murray and colleagues study, we developed separate models for sub-Saharan Africa and outside of Africa (with the exception of South Africa, which was modelled with countries outside of Africa, given the low malaria endemicity), age under 5 years and 5 years or higher, and males and females.

For countries that have only or mainly Plasmodium vivax transmission we used the number of deaths by year and age from vital registration data as a simple predictor of malaria mortality using a negative binomial regression model.

We estimated malaria cases separately for three sets of countries, which were divided on the basis of the availability and quality of malaria incidence data (see appendix pp 241-42 for the list of countries). The first group contained countries with unavailable or unreliable malaria case reporting systems. We estimated malaria incidence in these countries using a mortality-incidence model, in which we predicted malaria incidence by regressing the log- 
transformed study-level incidence on the log-transformed malaria mortality rate, age-group indicators, a sub-Saharan Africa indicator, an indicator distinguishing active versus passive case detection (set to active when generating predictions), and the ratio of the site-specific PfPR to national PfPR (from MAP 2010; set to the value 1 when generating predictions so that the estimates are nationally representative). In this model, the incidence data came from available studies and the mortality data came from our CODEm analysis. The second group included countries for which there were incomplete administrative data, for which we predicted malaria incidence by regressing incidence data from the World Malaria Report 2013 on national-level PfPR. We corrected for underreporting using a composite indicator for health system access as a proxy. The third group contained countries with complete and reliable administrative case reports, for which we used reported numbers as published in the World Malaria Report.

\section{CoDCorrect algorithm}

As with all causes of death analysed for the GBD, we require that the sum of each individual cause of death for a country, age, sex, and year equals the estimate of all-cause mortality. The CoDCorrect algorithm rescales the sum of causes at the individual draw level. The effect of this simple algorithm is to change causes that have larger uncertainty intervals if there is a mismatch between the sum of cause-specific mortality and all-cause mortality. To preserve the relations between incidence, prevalence, and death that come from the Spectrum analysis, the entire epidemic curve for HIV is scaled in CoDCorrect.

\section{Role of the funding source}

The sponsor of the study had no role in study design, data collection, data analysis, data interpretation, or writing of the report. The authors had access to the data in the study and the final responsibility to submit the paper for publication.

\section{Results}

Figure 7 shows the estimated trend in global numbers of incident cases, people living with HIV (prevalence), and deaths from HIV. Global HIV incidence peaked in 1997 with 2.8 million (95\% uncertainty intervals 2.7 to 3.1 ) new infections and has since decreased at $2.7 \%$ (2.0 to 3.1) per year. From 1997 to 2005, incidence decreased at 3.8\% (3.0 to 4.6$)$ per year and from 2005 to 2013 at $1.6 \%$ ( 0.6 to 2.4$)$ per year. New infections in children decreased from 340000 (323000 to 363000 ) in 2000 to 134000 (123000 to 152 000) in 2013 at an annualised rate of change (ARC) of $-7.2 \%(-7 \cdot 8$ to $-6 \cdot 4 \%)$, while new infections in adults decreased from 2.3 million ( 2.1 million to 2.4 million) to 1.7 million ( 1.6 million to 2.0 million), falling at $2.4 \%$ (1.6 to 3.0) per year, on average, during this period. Annual incidence estimated by UNAIDS is uniformly higher in the years shown and shows a sharper rate of decrease. Prevalence of individuals infected with HIV has steadily risen to 29.2 million (28.1 million to 31.7 million) in 2013 rising more rapidly from 1990 until about 2000 at an annual rate of change of $10.6 \%$ (10.1 to $11 \cdot 3)$, and increasing at $1.2 \%$ ( 0.9 to 1.4$)$ per year since (figure 7). Compared with UNAIDS's estimations, our estimation for 2012 suggest 6.6 million fewer individuals living with HIV. 32.0\% of this difference is in subSaharan Africa and $68.0 \%$ is elsewhere. Figure 7 shows trends for HIV deaths compared 
with UNAIDS estimations, with a peak mortality of 1.7 million (1.6 million to 1.9 million) in 2005 . Annual mortality has subsequently fallen to 1.3 million ( 1.3 million to 1.5 million) in 2013, an ARC of $-3.1 \%$ (-4.0 to 2.2). While the time trend of mortality estimated by UNAIDS is similar to ours, the estimated number of deaths is substantially greater. At the peak of the epidemic in 2005, our revised assessment of the HIV epidemic suggests 635000 fewer deaths than UNAIDS's estimates, although the difference (240 000) narrows substantially by 2012 , the last year available from UNAIDS. For the interval 2005-12, the UNAIDS ARC for death numbers was $-5.0 \%$ ( -5.6 to -4.5$)$, reflecting the lower assumed death rates on ART in the UNAIDS version of Spectrum.

New HIV infections are concentrated in young adults and to a much lesser extent, in children under 5 years of age (figure 8 ); $4.1 \%$ (3.8 to 4.5 ) of new infections occur in idividuals older than 60 years. New cases in 2013 occurred equally in both sexes. However, there are more infections in women than men at ages 15-24 years. Incidence in children, and in older adults, is similar for both sexes. Showing a mean survival time of more than 10 years in most countries and age-groups, the age pattern of deaths peaks in women at ages 35-39 years and in men at 40-44 years (figure 8 ). More deaths are in male individuals (53.9\% [51.9 to 56.1]) than in female individuals. The proportion of deaths that occur beyond age 60 years $(6 \cdot 8 \%$ [6.2 to 7.4]) is larger than the proportion of incident cases that occur beyond age 60 years $(4 \cdot 1 \%$ [3.8 to $4 \cdot 5])$.

Table 2 shows the ARCs for 1990-2000 and 2000-13 for incidence, prevalence, and death for the $21 \mathrm{GBD}$ regions and the world. ARCs between two fixed time periods need to be interpreted bearing in mind that measures of the HIV epidemic such as incidence and death will have peaked and decreased in particular countries at different times during the interval. Nevertheless, our findings show the accelerated progress in most of the world's regions. The only regions with a reversal in the ARC such that incidence was decreasing but in the later period is increasing or stagnating are southeast Asia, high-income North America, western Europe, Australasia, and Tropical Latin America. The reversal in southeast Asia can be explained by the large decrease in the 1990s achieved by a successful campaign to reduce infection through commercial sex encounters in Thailand, the country in the region with the largest epidemic. The reversal of incidence trends in North America might show a wearing off of the effect of public health measures to reduce the risk of transmission in men who have sex with men.

Age-standardised HIV incidence rates per 100000 population (figure 9) in 2013 ranged from less than 0.7 in a group of countries ringing the Eastern Mediterranean, parts of northern and central Europe, and Mongolia, to more than 570 in South Africa, Lesotho, and Swaziland. HIV infection follows distinct geographic patterns with continued high levels of infection throughout eastern and southern Africa, with some exceptions. Rwanda, Burundi, DR Congo, Congo, and Gabon all have incidence rates less than 120 per 100000 population, lower than their neighbouring countries. Incidence rates vary widely in subSaharan Africa, but are more homogenous across countries in Asia as well as North and South America. Important exceptions to these patterns include incidence rates above 15 per 100000 population in many Caribbean countries. Incidence rates are notably higher in Portugal and Ukraine, as well as Russia and some Central Asian republics. Figure 9 shows 
prevalence rates in countries in 2013. Geographical patterns are similar to incidence, although some differences are noteworthy. Prevalence levels are highest in Botswana, Lesotho, and Swaziland (above 12000 per 100000 population). There is substantial variation within sub-Saharan Africa; prevalence rates in Botswana, for example, are 15 times higher than in the DR Congo and 40 times higher than in Niger. In southeast Asia, prevalence is substantially higher in Thailand and Papua New Guinea. Prevalence rates are comparatively high in parts of Europe and central Asia (Portugal, Spain, Ukraine, Russia, and Kazakhstan) and in Latin America and the Caribbean (Panama, Honduras, Belize, Guatemala, Guyana, Suriname, Haiti, Dominican Republic, Jamaica, and Bahamas) prevalence levels exceed 220 per 100000 population. Comparison of incidence and prevalence in figure 9 draws attention to some differences for countries in their comparative ranking, such as for Sweden and Australia. Cross-national variations in HIV mortality rates per 100000 population, largely mirror the pattern reported for prevalence, varying from less than 0.2 in northern and central Europe and the Eastern Mediterranean, to more than 520 in southern Africa, a roughly 2500-fold difference (figure 10). Table 3 shows more detail on the estimated number of new infections and deaths in 2013 for both sexes, individually and combined, for 188 countries, along with ARCs in age-standardised incidence and death rates for both sexes combined.

By comparing the estimated number of person-years lived in a no-intervention scenario with actual estimates, we can compute the years of life saved through ART, PMTCT, and cotrimoxazole prophylaxis. Figure 11 shows cumulative years of life saved by GBD region as a result of these interventions during three phases of scale-up. From 1990 to 2003, 1.5 million years of life (1.2 million to 1.9 million) were saved, of which only $22.7 \%$ (14.2 to $32 \cdot 1$ ) were in populations living in developing countries, largely in Brazil. The number of years of life saved increased substantially in the period 2004 to 2008 to 3.9 million ( $3 \cdot 2$ million to 4.7 million), and the share in populations in developing countries increased to $52.6 \%$ (44.1 to 62.2). Between 2009 and 2013, the number of life-years saved was 13.7 million (11.8 million to 15.7 million). A much greater share (40.8\% [33.8 to 47.6]) of these life-years saved were in eastern and southern sub-Saharan Africa, and a further 12.1\% (9.0 to 15.7) in western sub-Saharan Africa. Other regions to benefit substantially from HIV interventions include high-income North America, western Europe, and south Asia. The number of years of life saved continues to grow rapidly due both to the continued expansion of ART and the cumulative effect of infections prevented in children. By 2013, the global cumulative number of years of life saved was 19.1 million (16.6 million to 21.5 million); $14.2 \%$ (12.4 to 16.2$)$ at ages younger than 15 years, $49.7 \%$ (45.8 to 53.4$)$ at age $15-49$ years, and $36 \cdot 1 \%$ (32.7 to 39.5$)$ at 50 years of age or older.

Since 2000, cumulative DAH for HIV up through 2011 totals $\$ 51.6$ billion, of which $\$ 32.7$ billion can be traced to specific developing country programmes in 2011 US dollars. ${ }^{109}$ Comparison of the total amount invested in HIV prevention and treatment to the years of life saved during 2000-11 yields in developing countries a ratio of $\$ 4498$ per life-year saved. The ratio of DAH for HIV to years of life saved varies widely from \$2.38 in Uruguay per life-year saved to $\$ 1.87$ million in Mongolia per life-year saved. 
The scale-up of ART has been variable across countries. Because of the temporal dynamics of the epidemic in different countries, comparisons of intervention scale-up are confounded by the timing of incidence. Nevertheless, the appendix ( $\mathrm{p} 22$ ) shows a crude comparison of years of life saved over the age of 15 years divided by prevalent cases in people older than 15 years in 2013. This ratio ranges from less than 0.07 in countries with minimal intervention to more than 0.49 in many high-income countries. In developing countries, Brazil stands out with a ratio of 0.37 . In the next tier, with ratios between 0.28 and 0.35 includes many countries in Latin America and Botswana, Namibia, Thailand, Cambodia, South Korea, and some countries in central Europe. In eastern and southern Africa, Ethiopia, Rwanda, and Burundi we saw higher ratios than in many of their neighbouring countries. Pronounced variation within regions points to the historical variation in the timing of the epidemic response.

Figure 12 shows a comparison of our estimated prevalence for country-age-sex groups against national population prevalence surveys. This comparison provides a rough check that at the end of the modelling process our assessment remains consistent with populationbased prevalence measurements as well as being consistent with data for all-cause mortality. In general, there is a strong correlation (0.96) of our country-age-sex estimates with survey prevalence-UNAIDS prevalence is also correlated (0.96) with survey data. However, in $21 \%$ of cases, there is a statistically significant difference (19\% for UNAIDS). The coefficients obtained by regressing both GBD and UNAIDS estimates on survey estimates showed that both methods tended to be slightly higher than the surveys; however, only the UNAIDS coefficient was a statistically significantly larger than one: UNAIDS, 1.08 (1.03 to 1.13); GBD, 1.02 (0.98 to 1.06). Country-specific graphs comparing GBD, UNAIDS, and survey prevalences by age and sex are shown in the appendix ( $\mathrm{p} 24)$.

Figure 13 shows the temporal changes of tuberculosis incident case numbers, the number of prevalent cases, and the number of deaths from 1990 to 2013. Total tuberculosis numbers are shown as well as numbers for tuberculosis in individuals who are HIV-negative. The number of incident cases for tuberculosis in individuals who are HIV-negative has increased from 5.0 million ( 4.8 million to 5.1 million) in 1990 to 7.1 million (6.9 million to 7.3 million) in 2013-a 1.5\% (1.4 to 1.6) annual change. Prevalence in 1990 and 2013 was 1.6 times higher than incidence, implying a duration of 20 months on average for a case. Prevalence rates increased slightly between 1990 and 2000 (ARC 0.4\% [0.2 to 0.6]) but decreased by $1.3 \%$ (1.4 to 1.2) per year from 2000 to 2013. Deaths from tuberculosis in individuals who are HIV-negative are decreasing at a faster rate, from 1.8 million (1.7 million to 1.9 million) in 1990 to 1.3 million ( 1.2 million to 1.4 million) in $2013-\mathrm{a}-1.4 \%$ $(-1.9$ to -1.0$)$ annual change. Decreases in death numbers and increases in incidence numbers implies that the case-fatality rate has been falling over the period; the ratio of deaths to incidence overall went from $0.36(0.33$ to 0.39$)$ in 1990 to $0.18(0.16$ to 0.20$)$ in $2013-\mathrm{a}-2.9 \%(-3.4$ to -2.5$)$ per year rate of change. Most global tuberculosis incidence cases and deaths in individuals who are HIV-negative are in men and boys, 64.0\% (63.6 to 64.3) for incidence and 64.7\% (60.8 to 70.3) for mortality (figure 14). Although age-specific rates rise with age up to 70 years, in view of the comparatively young age-structure of countries with substantial burden of tuberculosis in individuals who are HIV-negative, 
$83.2 \%(82.6$ to 83.8$)$ of cases and $58.8 \%(56 \cdot 7$ to $60 \cdot 6)$ of deaths were in people younger than 60 years in 2013 .

Table 4 shows a summary at the global and regional level of the ARCs for age-standardised rates of incidence, prevalence, and deaths for tuberculosis in individuals who are HIVnegative (see the appendix p 203 for tuberculosis including HIV-positive individuals). At the global level, age-standardised mortality rates decreased by $3.3 \%$ ( $4 \cdot 1$ to $2 \cdot 6)$, whereas incidence remained stable $(0.0 \%[-0.2$ to 0.2$])$ and prevalence rates increased by $0.4 \%(0.2$ to 0.6 ) during the period 1990 to 2000 . Global decreases continued in the period 2000 to 2013 for mortality $(-3.7 \%$ [ -4.4 to $-3 \cdot 0])$ whereas incidence decreased by $0.6 \%(0 \cdot 7$ to $0 \cdot 5)$ and prevalence decreased by $1.3 \%$ (1.4 to 1.2). Across regions, in the period 2000 to 2013, incidence rate ARCs in individuals who are HIV-negative ranged from $0.8 \%$ (0.6 to 1.0$)$ in Oceania to $-3.3 \%$ ( -3.6 to $-3 \cdot 1)$ in high-income North America. 16 of 21 regions had a greater decrease (or at least a smaller increase) in the incidence rate from 2000 to 2013 than for 1990 to 2000. Mortality rate decreases in HIV-negative individuals were greater in the period 2000 to 2013 than the decreases in prevalence in all 21 regions. The global decline in prevalence from 2000 to 2013 is largely accounted for by the large decreases in just two regions: east and south Asia. In south Asia, which accounts for 34.8\% (33.9 to 35.6) of incident cases and $47.7 \%$ (43.5 to 51.8) of deaths in 2013, the ARCs for 2000 to 2013 were $-1.1 \%(-1.3$ to -0.8$)$ for incidence, $-2.4 \%(-2.7$ to $-2 \cdot 2)$ for prevalence, and $-4.2 \%(-5 \cdot 6$ to -2.9) for mortality. Accelerated decreases in prevalence, incidence and mortality occurred in east Asia from 2000 to 2013 : $-3 \cdot 2 \%(-3 \cdot 4$ to $-2 \cdot 9)$ for prevalence, $-2 \cdot 1 \%(-2 \cdot 4$ to $-1 \cdot 9)$ for incidence, and $-7.5 \%(-8.5$ to -6.6$)$ for mortality. The tuberculosis trend in eastern Europe has reversed: in the 1990s, mortality, incidence, and prevalence rates were all increasing, with ARCs of $8.3 \%$ (5.5 to 9.1 ), $1.3 \%$ (1.0 to 1.7 ), and $1.7 \%$ (1.4 to 2.0$)$, respectively. However, in the period 2000 to 2013 the trends for all three of these indicators have improved, with ARCs of $-4.8 \%(-7.6$ to -3.9$),-0.6 \%(-0.8$ to -0.4$)$, and $-0.7 \%(-1.0$ to $-0 \cdot 5)$. Table 5 shows incidence and deaths by country in 2013 along with ARCs for agestandardised rates.

Figure 15 shows maps of age-standardised incidence rates and death rates for tuberculosis in individuals who are HIV-negative in 2013. Age-standardised incidence of tuberculosis in individuals who are HIV-negative is more than 200 per 100000 population in 24 countries in sub-Saharan Africa as well as in North Korea, Timor-Leste, Cambodia, Laos, Philippines, and Kiribati (figure 15). There are an additional 16 countries with rates of more than 150 per 100000 population that include Bangladesh, Myanmar, India, Pakistan, Ethiopia, and Malawi. Figure 15 also shows tuberculosis death rates in individuals who are HIV-negative which are above 10 per 100000 population in all countries in sub-Saharan Africa and increase to more than 50 per 100000 population in 40 countries. Among middle-income countries outside of sub-Saharan Africa, Afghanistan, Indonesia, India, Myanmar, and the Philippines stand out as countries with death rates higher than 50 per 100000 population. The highest age-standardised death rates in Latin America and the Caribbean are in Haiti followed by Bolivia and Peru. Death rates per 100000 population are highly variable in north Africa and the Middle East, ranging from very low in Syria (0.5 [0.3 to 0.9]) and Jordan $(0.8$ [0.6 to 1.0$])$ to high in Morocco [14.3 [11.2 to 17.5]) and Yemen (19.9 [11.5 to 41.5]) in 2013. Eastern Europe and central Asia stand out with death rates that are 
substantially higher than western or central Europe. China has lower rates of death than eastern Europe and central Asia. Our findings are mostly consistent with the list of highburden countries used by WHO; however, our top 22 countries in terms of case numbers or death numbers that are not on the WHO high-burden list include South Korea, North Korea, and Madagascar for case numbers and Angola and Zambia for mortality. WHO high-burden countries that did not make our top 22 list for case numbers include Afghanistan, Cambodia, and Tanzania, and the WHO high-burden countries that did not make our top 22 list for mortality include Brazil and Cambodia.

Trends in the annual number of new cases of malaria, and annual deaths from malaria, are shown in figure 16 for the period since 1990. Global incidence seems to have peaked in 2003 at 232 million cases (143 million to 387 million) and has since fallen by about $29 \%$ to 165 million new cases (95 million to 284 million) in 2013. There is massive uncertainty around these estimates: the 2013 estimate, for example, could be anywhere between 95 million and 284 million. The estimates of new malaria cases in the World Malaria Report fall within the uncertainty intervals of the GBD estimates with a mean number of new cases in 2012 of 207 million (135 million to 287 million). By contrast with incidence data, the World Malaria Report estimates of malaria deaths are slightly lower (figure 16). There are also some important differences in the timing of the peak and decline in malaria mortality. Annual malaria deaths began to decline from a peak of 1.2 million (1.1 million to 1.4 million) in 2004 to about 855000 (703 000 to 1032 000) in 2013, having increased from 888000 (793 000 to 993000 ) in 1990. According to the World Malaria Report, ${ }^{110}$ malaria caused about 627000 deaths in 2012, having reached a peak of about 900000 around the turn of the century. The age-sex pattern of global malaria incident cases and deaths in 2013 is shown in figure 17. The largest number of cases is in people younger than 15 years. Malaria deaths, by contrast, are concentrated in children younger than 5 years, although malaria caused between 10000 and 25000 deaths in each 5-year age group beyond age 15 years, so that the cumulative fraction of malaria deaths in adults reaches $33.75 \%$.

Globally, age-standardised malaria incidence and death rates were increasing in the period 1990-2000 (table 6), but many regions outside of sub-Saharan Africa and north Africa and the Middle East had decreases in age-standardised incidence, death rates, or both. In the period since 2000, all regions including sub-Saharan Africa had decreases in agestandardised incidence and death rates. Incidence decreased by $38 \%$ (37 to 40 ) in central Asia since 2000, a result of increased malaria elimination efforts in the region. Figure 18 shows the geographical distribution of the age-standardised incidence rate in 2013. The geographical distribution of the age-standardised mortality rate (figure 18) shows a similar pattern with the highest rates in Mozambique, Burkina Faso, Guinea-Bissau, Mali, Sierra Leone, The Gambia, and Guinea. Rwanda stands out as having low rates compared with its neighbouring countries. Outside of Africa, Yemen, India, Myanmar, and Papua New Guinea have death rates higher than 7.5 per 100000 population. In southeast Asia, Thailand and Malaysia have achieved very low death rates. Table 7 shows incidence and death numbers by country in 2013 along with ARCs for age-standardised rates. Four countries have over 5 million cases a year including India with over 60 million cases, Nigeria (30 million), DR Congo (with 6 million), and Mozambique (6 million). Three countries-Nigeria, DR Congo, and India—account for roughly 50\% of all malaria deaths in 2013. 


\section{Discussion}

HIV, tuberculosis, and malaria remain major health challenges in 2013. The mean age of death differs substantially between them, at 15.3 years for malaria, 38.6 years for HIV, and 52.9 years for tuberculosis in HIV-negative individuals, which means that the burden in terms of years of life lost varies across the diseases. Tuberculosis deaths have decreased globally since 1990, and after 2000 incidence, prevalence, and death have all decreased. HIV incidence peaked in 1997 and mortality peaked in 2005 with substantial declines since the peak in each. Malaria incidence and mortality peaked and began declining in 2004 with substantial drops in the number of child deaths in sub-Saharan Africa over the past 5 years. There is substantial variation both in levels and trends for all three diseases across countries. HIV and malaria incidence and death are concentrated in sub-Saharan Africa whereas tuberculosis burden is more widespread but most pronounced in south and southeast Asia.

From our analysis of HIV data, our findings show that the HIV epidemic is smaller than estimated by UNAIDS. Our global epidemic curve for mortality ranges was lower than estimated by UNAIDS for every year; at the peak in 2005 our estimates are $27.0 \%$ lower and in 2012 are $14.5 \%$ lower. Our estimates of global prevalence differ from UNAIDS's by $17.1 \%$ in 2005 and $18.7 \%$ in 2012 . The substantial differences in the number of deaths stem from two key differences in these analyses. First, in the 125 concentrated epidemics with some cause of death data for mortality due to HIV, our estimated mortality is $52.2 \%$ lower in 2000 and $58.4 \%$ lower in 2012 than UNAIDS's estimates. Our prevalence estimates are, for example, 36.3\% lower for Panama, 52.2\% lower for Colombia, and 58.4\% lower in Russia. Second, in the large generalised epidemics, selecting epidemic curves that are consistent with prevalence data, all-cause mortality, and available studies on survival with and without ART shifted median survival up. For example, in southern Africa, median survival off ART for the age-group 25-34 years increased from 10.5 years to 11.5 years. Longer or shorter survival off ART in some countries could be explained by genetic factors, ${ }^{11-115}$ co-factors such as the presence of other diseases like malaria, ${ }^{116-118}$ differential access to treatments for opportunistic infections, or other co-factors that have not been described. These findings are important in terms of identifying the magnitude and comparative burden of HIV. Table 8 outlines the differences between our HIV/AIDS estimation strategy and that of UNAIDS.

Comparison of population-based surveys with our estimates of prevalence suggest reasonable alignment and the regression analysis of estimated prevalence on measured prevalence suggest there is not systematic tendency in our estimates to overestimate or under estimate prevalence. However, much variation exists by age and sex with nearly one in five of our age-sex specific prevalence estimates statistically different than the survey prevalences. Several potential explanations for this variation exist. Our assumptions about the relative incidence pattern by country might not be true at the local level. Differential non-response in the surveys by age and sex is also a potential factor. The adjustments made through the demographic matching and CoDCorrect algorithm could contribute to the differences. More analysis on a country-by-country basis will be helpful in exploring these issues in future research. 
Revisions of the global epidemiology of HIV of this magnitude-in view of the weakness of direct measurement of incidence and death—should not be surprising. As prevalence surveys became more widely available, UNAIDS revised downward their global prevalence estimates by $18 \%$ in 2007 and their global mortality estimates by nearly $24 \% .{ }^{119}$ Taking into account more data for survival on and off ART and incorporating all-cause mortality data has led to revisions of a similar magnitude. Our revisions also suggests that there is greater uncertainty for incidence, prevalence, and death than previously estimated. Irrespective of the specific estimates generated from imperfect data, however, our assessment of prevalence continues to point to the very large and steadily growing numbers of infected individuals, many of whom are in need of antiretroviral therapy. Great progress has been achieved reducing infections in children (62.4\% reduction since the incidence peak in 2002) due to the scale-up of interventions. The continued 1.7 million new infections per year in adults, down $32.7 \%$ from the peak of the epidemic at the global scale, however, is a stark reminder of the continuing epidemic.

A key finding that confirms many local, regional, and global studies ${ }^{120-125}$ is that interventions, especially ART, PMTCT, and co-trimoxazole, have had a profound effect. Cumulatively, 19.1 million years of life have been saved since 1996, 5.7 million in developed countries and 13.4 million in developing countries, where the ratio of DAH to years of life saved is less than $\$ 4500$ for the average of the period 2000-11. In view of the very rapid increase in years of life saved in 2012 and 2013, the ratio for the period 2000-13 when DAH figures are available will probably be much lower. The scale-up, number of lives saved, and comparatively low price per year of life saved is one of the major achievements in global health in the past decade. Many groups—local, national, and global-deserve credit for this accomplishment. DAH does not count national contributions to the cost of HIV programmes; real variation in the ratio of the total cost per year of life saved is probably much smaller because many middle-income countries receive little DAH and fund most HIV interventions from their own resources. Micro-economic studies of the cost per years of life saved have also reported wide variation across locations. ${ }^{126-128}$ We would expect, given investments in initial programme start-up including capital equipment investments, that the ratio of DAH to life-years saved will decrease over time. With prevalence growing $5.8 \%$ per year over the past 5 years, the need to learn from more efficient programmes is paramount. Our analysis of survival on ART shows wide variation in programme outcomes within sub-Saharan Africa. Counterfactual analysis of what might happen if all programmes achieved the levels of mortality seen in the programmes with the best outcomes or even what would happen if high-income country on-ART death rates were achieved would help shed further light on the importance of quality improvement for future HIV death reductions. Improving cost-effectiveness of ART programmes will require a process of continuously documenting costs, outcomes, and efficiency along with a mechanism for shared learning across programmes on improving quality. HIV infected intravenous drug users have not benefited as much from treatment as those infected through sexual transmission. Regions with an ongoing increase in mortality from HIV in the 200013 period are high-income Asia Pacific, central and eastern Asia, eastern and central Europe, north Africa and the Middle East, Oceania, and southern and western sub-Saharan. In a number of these regions large proportions of HIV cases are in intravenous drug users for 
whom countries might be less inclined to provide treatment services. Even in countries with a greater emphasis on harm-reduction strategies, drug users might still be a more difficult group for health services to reach. ${ }^{129}$

Age-standardised tuberculosis mortality rates including tuberculosis in HIV-positive individuals at the global level changed at $-2.8 \%(-3.6$ to -2.2$)$ per year from 1990 to 2000 and around a percentage point faster from 2000 to $2013(-3.7 \%$ [ -4.4 to $-3.0 \%])$ per year. When examining tuberculosis mortality in individuals who are HIV-negative, the acceleration was smaller, from $-3 \cdot 3(-4 \cdot 1$ to $-2 \cdot 6)$ to $-3 \cdot 7 \%(-4 \cdot 4$ to $-3 \cdot 0)$ change per year but still statistically significant. There has been comparatively little decrease in the global age-standardised tuberculosis incidence rates in HIV-negative individuals although some regions such as south Asia and east Asia have seen accelerated declines since 2000.

Prevalence has decreased much faster than incidence, which is consistent with earlier and more effective treatment-shortening durations. In addition to shorter duration, the death to incidence ratio changed from 0.36 in 1990 to $0 \cdot 18$ in 2013, also a likely consequence of treatment. There has been much regional and country variation in progress on tuberculosis with the ARC for mortality ranging from $-10.3 \%$ to $2.5 \%$ from 1990 to 2013 and the ARC for incidence ranging from $-3.3 \%$ to $2.5 \%$ over the same period. This variation implies that more rapid progress is possible at the global scale if lessons can be learned from countries with more rapid achievement. Since 2000, as for HIV and malaria, global progress in terms of prevalence and mortality has accelerated. We are unable to compute the extra years of life saved for tuberculosis as we can for HIV; but the comparatively small DAH for tuberculosis over the period 2000-11 ( $\$ 8.3$ billion) has been associated over this time period with greater reductions in incidence, prevalence, and death rates. Tuberculosis is different from HIV and malaria in that the rising incidence and death rates with age mean that demographic ageing of the world's population in the absence of other changes will naturally lead to higher numbers of cases and deaths. Demographic changes in essence slow the progress of tuberculosis control; a factor that should be built into considerations of funding and programme strategy. The established links between alcohol, diabetes, tobacco smoking and tuberculosis also mean that trends in these risk factors can modulate trends in tuberculosis. ${ }^{131-133}$ In this analysis, we have not separately examined the incidence, prevalence, and mortality related to multi-drug resistant tuberculosis (MDR-TB). There are concerns that even in places with substantial decreases in tuberculosis incidence, prevalence, and death such as in China, MDR-TB might be a substantial challenge. ${ }^{82,133-136}$ Modelling studies have shown that under specific circumstances MDR-TB could reverse important gains made in combatting tuberculosis. ${ }^{82,136,137}$ Future revisions of the burden of disease should examine more carefully the evidence on the levels and trends in MDR-TB.

Our results for tuberculosis differ from WHO estimates in some important ways. In general, we estimate higher mortality, lower prevalence and incidence, and a smaller fraction of tuberculosis related to HIV. Our estimates of prevalence are driven by the available prevalence surveys and are not back calculated from incidence. Our incidence estimates start with case-notifications corrected for missing age-groups and case types such as smearnegative pulmonary or extrapulmonary and expert judgment of the case-detection rate. For some countries, with implausibly large numbers of smear-negative and extra-pulmonary cases notified in individuals younger than 15 years, we have excluded these data from the 
analysis. However, the Bayesian meta-regression identifies a solution for incidence that is consistent with prevalence data and estimates of cause-specific mortality. Because this analysis is undertaken using age-specific and sex-specific rates, it also takes into account the changing relations between incidence, prevalence, remission, excess mortality, and causespecific mortality with age and sex. Because true incidence in any country is not known, our estimates as well as WHO's depend on expert judgment on the case-detection rate.

Systematic bias in the estimated case-detection rate, particularly for earlier time periods, will affect not only the volume of estimated tuberculosis cases but also time trends. India accounts for $27.1 \%$ (26.3 to 27.9) of global incident cases in 2013; systematic errors in the estimated incidence in India in the 1990s would have a profound effect on global trends. Perhaps more importantly, our assessment of global trends for death are similar to WHO but differ for prevalence and somewhat for incidence. Because total age-sex-specific case notifications reported to WHO for smear-positive pulmonary tuberculosis have continued to increase-by 1.1 million in 2000, 2.3 million in 2005, and 2.5 million in 2012-differences in time trends stem from assumptions about the case-detection rate and, in our study, the incorporation of information from prevalence surveys and all-cause mortality. Table 9 outlines the differences between our tuberculosis estimation strategy and that of WHO.

Malaria burden rose steadily until 2004 and has since decreased. The Global Fund, President's Malaria Initiative, and other bilateral and private initiatives have spent $\$ 11.3$ billion in DAH from 2000 to 2011. The hypothesis that global action has been an important factor in these declines is highly plausible. ${ }^{6,110,138-141}$ The decline in our assessment is driven by the statistical model fitted to the available but sparse verbal autopsy data. Key independent variables in the model that drive this estimated decline are resistance for firstline agents and ITN coverage. Noor and colleagues ${ }^{142}$ assessment of trends in PfPR from 2000 to 2010 indicate that some countries such as Malawi, Zambia, or DR Congo have had substantial scale-up of ITNs with minimal reduction or increases in PfPR. Explanations for the mismatch between ITN scale-up and changes in PfPR could lie in the estimation of any of PfPR, ITNs, or local factors that affect who actually receives and uses ITNs, or it could be a function of other factors-eg, climatic changes over this period. There could also be important threshold or saturation effects for ITNs on PfPR. While the substantial decline in child mortality in the past 5 years is welcome news, understanding the relative role of artemisin in combination treatment scale-up and vector control is challenging and might vary by country. The variability in the relation between ITNs and PfPR at the national level emphasises the risks of simply assuming that ITN scale-up at the national level will yield the percentage reduction in child deaths seen in the randomised trials; a strategy used by Child Health Epidemiology Reference Group (CHERG) in their child mortality estimates. It is also important to note that the CHERG models did not include a first-line drug resistance as a covariate. Our findings show this to be an important predictor in the Africa models in particular and a key driver of the temporal trend noted in this region. Table 10 outlines the differences between our malaria estimation strategy and that of WHO.

MDG6 brought global attention to these three diseases, ushering in an unprecedented focus on specific diseases in the broader development agenda. Despite many who questioned the wisdom of a focus on specific diseases, there has been accelerated progress on HIV, malaria, and tuberculosis since 2000. In the case of HIV, our estimation strategy allows for direct 
quantification of years of life saved, which have been substantial. For tuberculosis and malaria, we see accelerated reductions in deaths and cases compared with the decade before the Millennium Declaration. Rigorously assigning causality to these accelerations to the global collective action catalysed by the Millennium Declaration is beyond the scope of this paper and likely impossible in view of the data limitations. Nevertheless, as governments and the global community debate the nature, scope, and utility of setting new global targets post-2015, these findings should be taken into consideration.

Our comparative analysis of these three diseases shows pronounced differences in data gaps and measurement challenges. Antenatal clinic serosurveillance and population surveys in generalised epidemics have been a powerful tool for tracking evolution of the HIV epidemic with real data collected on an annual basis. Cause of death data in many countries with concentrated epidemics also provide a timely way to examine the effect of HIV. But, national data for the outcome of ART is weak. Measures such as retention in care and loss to follow-up are often incomplete and difficult to compare across facilities, programmes, and countries. UNAIDS and our modelling do not routinely use national data for treatment outcomes and depend on cohort or published studies. In view of the huge effect of ART on death and prevalence, more accurate and continuous monitoring of ART outcomes and costs must be a high priority. By contrast with HIV, tuberculosis treatment outcomes are highly standardised and reported to WHO at least through the end of treatment. However, real-time data for the time-trends of tuberculosis is hard to come by. Case notifications can only be interpreted by resorting to expert opinion on the case detection rate, and prevalence surveys are infrequent and require large sample sizes. A system analogous to the antenatal clinic serosurveillance for tuberculosis would be possible if new diagnostics emerge that can quantitatively assess load of bacilli in an infected individual. Pending such a technological advance, more frequent prevalence surveys and perhaps capture-recapture studies ${ }^{82}$ are the only direct measurement available to track evolution of the epidemic. In our study, ARC for incidence and prevalence 2000 to 2013 is correlated (0.93). Information about malaria clinical cases and deaths is much weaker than for HIV or tuberculosis. Case reports are very incomplete. Most deaths occur in places without vital registration systems. Verbal autopsy is widely believed to exaggerate malaria deaths, especially in adults, ${ }^{143-145}$ in view of the tendency to overdiagnose malaria in African hospital settings; ${ }^{146-149}$ our systematic review of validation studies, though, shows low sensitivity (33\%) and low specificity (93\%). Bias is a function of both sensitivity and specificity. If these validation studies that are published are correct we might be underestimating and not overestimating malaria deaths in adults and children in areas with substantial malaria and the reverse in areas with little malaria. The only comparatively easy-to-measure outcome related to malaria is the $P$ falciparum parasite rate. Local surveys have been usefully collated and analysed by Noor and colleagues and the Malaria Atlas Project. ${ }^{142}$ These data provide hard evidence on the trends in a measurable outcome; the challenge is that there is a loose relation in the available data between PfPR and incidence or mortality. In view of how important malaria is, the state of monitoring systems for malaria burden is poor. Repeated verbal autopsy studies combined with carefully designed validation studies would be helpful. Better data for the incidence of clinical episodes confirmed with rapid diagnostic tests and how it varies as a function of the PfPR would improve incidence estimation. 
Findings for these three diseases draw attention to the difference between ICD-assigned underlying cause of death and the total mortality attributable to a disease including pathways through which a disease aggravates other disorders. ICD rules treat disorders-not just these three diseases - in this regard in substantially different ways. All deaths directly related to pregnancy and childbirth and any deaths aggravated by pregnancy are counted as maternal deaths. For HIV, all deaths in individuals who are HIV-positive are assigned to HIV unless they are due to completely incidental causes such as a road traffic injury. Following this convention, tuberculosis deaths in HIV-positive people are assigned to HIV not tuberculosis. In the case of malaria, there has long been the recognition that malaria might increase the risk of death in children and adults from other causes such as septicaemia or chronic kidney disease. ${ }^{150-152}$ Early studies after the country-by-country elimination or rapid control of malaria documented rapid changes in deaths from pneumonia and chronic kidney disease, ${ }^{153,154}$ suggesting the full effects of malaria on mortality are greater than the ICDcoded malaria deaths. The ITN randomised controlled trials based on verbal autopsy documented that about half of the declines in under- 5 mortality were in causes other than malaria assigned through a verbal autopsy. ${ }^{155,156}$ Our understanding of the magnitude of each disease is affected by the at-times arbitrary rules governing assigning causes of death.

There are two important general observations from our analysis of HIV compared with prior analyses that might be relevant to other diseases. First, we saw that concentrated epidemics have been systematically over estimated by a factor on average of more than two. Overestimation is most likely related to a tendency to overestimate the size of high-risk groups for which little information exists. Why would expert judgment be, on average, so wrong? The disconnect in many countries between expert judgment and the results emerging from the analysis of cause of death data should caution researchers in the future from too much dependence on expert judgment in descriptive epidemiology. Second, we saw a systematic under-estimation of uncertainty in many countries in the UNAIDS analysis. Their assessment for South Africa, for example, had for 2010 an uncertainty interval with a coefficient of variation of 0.03 . Our assessment before matching on all-cause mortality had a coefficient of variation about six times higher (0.19) in the same year. There is a general tendency, we believe, in many modelling efforts to underestimate uncertainty when arbitrary assumptions about parameters are made. For example, the default assumptions for uncertainty in the UNAIDS Spectrum model is a coefficient of variation $0 \cdot 05$ for mortality on and off ART. We find from our empirical analysis coefficients of variation that range from 0.44 (in the age group 15-24 years and CD4 count greater than 500) to 1.00 (in people aged 45 years or older and a CD4 count greater than 500). In other words, uncertainty in these parameters seems to have been underestimated, with the real value approximately ten times larger. This is not a critique of the UNAIDS Spectrum modelling effort, rather a reminder that statistical analysis of parameter uncertainty often shows that we know much less than we think.

Our analysis of HIV in India based on the 2005-06 National Family Health Survey and antenatal clinic serosurveillance suggests that in 2002 there were 287000 (199000 to 377 000) deaths. Cause of death data, however, are available from several sources all pointing to substantially lower numbers of deaths than UNAIDS high-risk group analysis. Using data for 2001-03, investigators in the Million Death Study reported an estimated 59 000-140 000 
deaths in 2004. The urban Medical Certification of Causes of Death system recorded a peak age-standardised death rate in $6 \cdot 3$ per 100000 population, which is equivalent at the national level to 57000 deaths. We did not use these sources in our assessment; the substantial mismatch between our estimates draws attention to the need for improved understanding of causes of death in India.

Some global health efforts to develop robust estimates of the burden of disease sometimes end up using both empirical measurement on incidence, prevalence, and cause-specific mortality plus coverage of interventions and assumed effectiveness of interventions. The blending of real measurement of outcome and assumed mapping of interventions to outcome is justified because recent scale-up of interventions might not be accounted for in the sparse measurements that are available. We used the modified Spectrum model to map ART coverage into likely changes in mortality from HIV. These findings are lent support in some countries by measured declines in all-cause mortality or cause-specific mortality but in other countries are based entirely on the presumed relation between intervention roll-out and mortality. Many examples of such blending of data for outcome and intervention coverage exist: CHERG estimates of decreases in child deaths due to ITNs and Hemophilus influenza type B vaccine coverage are not based on any statistical relation but on the assumption that interventions will yield the decreases seen in randomised trials. These are reasonable assumptions but fundamental difference exist between observing the change in outcome as opposed to assuming the outcome has changed; this difference is not immediately evident in global health estimation efforts. It can yield circular analyses in which estimates are used by other authors to assess impact. In general, in the GBD 2013, we have sought to use largely empirical data and statistical associations seen in the data to make estimates. But for HIV in particular, we have used the approach embodied in Spectrum.

This analysis of data for HIV, tuberculosis, and malaria has many limitations in view of the ambition to track incidence, prevalence, and mortality for 188 countries from 1990 to 2013. First, ART estimates for 2013 are highly preliminary. Countries have reported ART scale-up through 2012 and provided estimates for 2013. Many of these estimates were aspirational and we have used growth rates over the 5 years 2008-12 to adjust these 2013 estimates. Second, we have not independently validated the country reports of ART scale-up. We have added to the uncertainty by randomly varying ART scale-up by sampling a uniform distribution from plus to minus $10 \%$ but this presumes that, on average, ART scale-up is not exaggerated. Third, we have used 102 studies of ART outcomes to inform our assumptions of death rates on ART. There was much variation across sites. More recent programmes might be achieving better outcomes than previous studies have shown if there has been shared learning across programmes. Available studies might also be biased towards better outcomes through the publication bias; poor programmes are unlikely to seek to publish their results. There was insufficient national data to use local information about each country on ART programmes. Our estimates might be biased up or down for a given country because local ART outcomes might be better or worse than the sub-Saharan Africa average. Future rounds of estimation will be substantially improved by more robust ART treatment outcome data obtained from nationally representative samples of patients on ART across a wider range of countries. Fourth, we have sought to find epidemic curves for the major 
generalised epidemics that are both consistent with available prevalence data and all-cause mortality data derived from sources such as vital registration or sibling histories in household surveys. The process of matching all-cause mortality draws and Spectrum outputs that are consistent also substantially reduces uncertainty. In view of the mismatch of these data sources, we are probably underestimating uncertainty in these countries. Fifth, we have not modified the UNAIDS assumptions for survival in children infected with HIV.

However, published studies from high-income countries pre-ART suggest much higher survival. ${ }^{157}$ Our estimates of death in the age-groups 5-9 years and 10-14 years might therefore be exaggerated. Sixth, our estimates of uncertainty for HIV could be underestimated because some of our uncertainty ranges for parameter inputs have been selected arbitrarily and true variation, for example, in age-sex patterns of incidence might be larger. The idea that variations in age-sex patterns of incidence might be larger is lent support by the number of age-sex-country-years in which our estimates of prevalence are different to those available from national prevalence surveys. Seventh, on the basis of debate over the burden of malaria in adults, we chose not to redistribute garbage code deaths onto malaria in verbal autopsy studies in adults, which led to a reduction in the estimated number of adult malaria deaths. Although this choice was informed by expert opinion, it was not based on any direct data. Even without the redistribution of garbage codes, the percentage of deaths occurring in adults in Africa is still high and has to be interpreted with caution in view of the potential for misclassification bias in verbal autopsies. Alternatively, the choice not to redistribute ill-defined codes onto malaria might bias our adult deaths downwards. Eighth, Noor and colleagues have published PfPR for 2000 and 2010. ${ }^{142}$ A full time-series of PfPR would be a useful covariate for modelling the burden of malaria. The Malaria Atlas Project is working on such time-series analysis of PfPR and when it is finalised it would strengthen the analysis of malaria trends. Ninth, our uncertainty intervals for malaria incidence and mortality incorporate sampling uncertainty, non-sampling un certainty, and model-specification uncertainty, but do not incorporate the uncertainty that can stem from misclassification biases in verbal autopsy. Uncertainty is probably underestimated due to the limitations of verbal autopsy for malaria in children and adults. Tenth, findings from other studies in countries such as The Gambia suggest substantial decreases in malaria in these settings; in our analysis of mortality, however, these types of studies have not been used. ${ }^{158,159}$ Eleventh, our analysis of tuberculosis assumes that local expert judgment about the case-detection rate is unbiased; this assumption, however, might be incorrect for countries with higher or lower case-detection rates. Twelfth, our uncertainty intervals for tuberculosis incidence and prevalence generated from DisMod-MR 2.0 are probably underestimated. The intervals are narrow because we have extensive data inputs for essentially all countries in the form of adjusted case-notifications and CODEm estimates of all-cause mortality. Although each data point has substantial uncertainty, the metaregression produces narrow estimates of the predicted mean value for an age-sex-countryyear because of the extensive and often consistent data. These intervals do not capture the potential for systematic error in some of the data-processing steps such as the use of the expert-based case-detection rate. Despite these important limitations, the GBD approach has many advantages, primarily because it is a comprehensive and clearly documented approach to disease burden estimation that examines all the available data and invests substantial 
effort into standardisation of definitions, data adjustments, and modelling across all diseases and injuries.

The focus of the global health community on action to reduce HIV/AIDS, tuberculosis, and malaria, enshrined in MDG6, was not only appropriate in 2000 at the Millennium Declaration, but is increasingly relevant now in view of the slow but important progress that disease control strategies have yielded, particularly since 2005. Much remains to be done, however: although evidence now exists that the implementation of known interventions is beginning to have an effect, it is probably less than is widely believed, or hoped. But these interventions are working, and need to be rapidly scaled up with more funding, more emphasis on national health system strengthening in key affected countries to increase access to them by the poor, and more targeted research to accelerate progress. What is also clear from this analysis as we enter the final phase of the MDG era is how little we reliably know in many countries to track progress. Rapidly reducing the massive uncertainty that surrounds the measurement of these diseases, particularly malaria, will be essential if we are to better monitor, and respond to, evidence about progress, or not, with their control.

\section{Supplementary Material}

Refer to Web version on PubMed Central for supplementary material.

\section{Authors}

Christopher J L Murray, Katrina F Ortblad, Caterina Guinovart, Stephen S Lim, Timothy M Wolock, D Allen Roberts, Emily A Dansereau, Nicholas Graetz, Ryan M Barber, Jonathan C Brown, Haidong Wang, Herbert C Duber, Mohsen Naghavi, Daniel Dicker, Lalit Dandona, Joshua A Salomon, Kyle R Heuton, Kyle Foreman, David E Phillips, Thomas D Fleming, Abraham D Flaxman, Bryan K Phillips, Elizabeth K Johnson, Megan S Coggeshall, Foad Abd-Allah ${ }^{\dagger}$, Semaw Ferede ${ }^{\dagger}$, Jerry P Abraham ${ }^{\dagger}$, Ibrahim Abubakar ${ }^{\dagger}$, Laith J Abu-Raddad ${ }^{\dagger}$, Niveen Me AbuRmeileh $^{\dagger}$, Tom Achoki ${ }^{\dagger}$, Austine Olufemi Adeyemo ${ }^{\dagger}$, Arsène Kouablan Adou ${ }^{\dagger}$, José C Adsuar ${ }^{\dagger}$, Emilie Elisabet Agardh ${ }^{\dagger}$, Dickens Akena ${ }^{\dagger}$, Mazin J Al Kahbouri ${ }^{\dagger}$, Deena Alasfoor ${ }^{\dagger}$, Mohammed I Albittar ${ }^{\dagger}$, Gabriel Alcalá-Cerra ${ }^{\dagger}$, Miguel Angel Alegretti ${ }^{\dagger}$, Zewdie Aderaw Alemu ${ }^{\dagger}$, Rafael Alfonso-Cristancho ${ }^{\dagger}$, Samia Alhabib ${ }^{\dagger}$, Raghib Ali ${ }^{\dagger}$, Francois Alla ${ }^{\dagger}$, Peter J Allen ${ }^{\dagger}$, Ubai Alsharif ${ }^{\dagger}$, Elena Alvarez ${ }^{\dagger}$, Nelson AlvisGuzman $^{\dagger}$, Adansi A Amankwaa ${ }^{\dagger}$, Azmeraw T Amare ${ }^{\dagger}$, Hassan Amini ${ }^{\dagger}$, Walid Ammar ${ }^{\dagger}$, Benjamin O Anderson ${ }^{\dagger}$, Carl Abelardo T Antonio ${ }^{\dagger}$, Palwasha Anwari ${ }^{\dagger}$, Johan Ärnlöv ${ }^{\dagger}$, Valentina S Arsic Arsenijevic ${ }^{\dagger}$, Ali Artaman ${ }^{\dagger}$, Rana J Asghar ${ }^{\dagger}$, Reza Assadi $^{\dagger}$, Lydia S Atkins ${ }^{\dagger}$, Alaa Badawi ${ }^{\dagger}$, Kalpana Balakrishnan ${ }^{\dagger}$, Amitava Banerjee $^{\dagger}$, Sanjay Basu ${ }^{\dagger}$, Justin Beardsley ${ }^{\dagger}$, Tolesa Bekele ${ }^{\dagger}$, Michelle L Bell ${ }^{\dagger}$, Eduardo Bernabe ${ }^{\dagger}$, Tariku Jibat Beyene ${ }^{\dagger}$, Neeraj Bhala ${ }^{\dagger}$, Ashish Bhalla ${ }^{\dagger}$, Zulfiqar A Bhutta $^{\dagger}$, Aref Bin Abdulhak ${ }^{\dagger}$, Agnes Binagwaho ${ }^{\dagger}$, Jed D Blore ${ }^{\dagger}$, Berrak Bora Basara $^{\dagger}$, Dipan Bose ${ }^{\dagger}$, Michael Brainin ${ }^{\dagger}$, Nicholas Breitborde ${ }^{\dagger}$, Carlos A CastañedaOrjuela $^{\dagger}$, Ferrán Catalá-López ${ }^{\dagger}$, Vineet K Chadha ${ }^{\dagger}$, Jung-Chen Chang ${ }^{\dagger}$, Peggy PeiChia Chiang ${ }^{\dagger}$, Ting-Wu Chuang ${ }^{\dagger}$, Mercedes Colomar ${ }^{\dagger}$, Leslie Trumbull Cooper ${ }^{\dagger}$, Cyrus Cooper ${ }^{\dagger}$, Karen J Courville ${ }^{\dagger}$, Benjamin $\mathrm{C} \mathrm{Cowie}^{\dagger}$, Michael H Criqui ${ }^{\dagger}$, Rakhi 
Dandona $^{\dagger}$, Anand Dayama ${ }^{\dagger}$, Diego De Leo ${ }^{\dagger}$, Louisa Degenhardt ${ }^{\dagger}$, Borja Del PozoCruz $^{\dagger}$, Kebede Deribe ${ }^{\dagger}$, Don C Des Jarlais ${ }^{\dagger}$, Muluken Dessalegn ${ }^{\dagger}$, Samath D Dharmaratne $^{\dagger}$, Uğur Dilmen ${ }^{\dagger}$, Eric L Ding ${ }^{\dagger}$, Tim R Driscoll ${ }^{\dagger}$, Adnan M Durrani ${ }^{\dagger}$, Richard G Ellenbogen ${ }^{\dagger}$, Sergey Petrovich Ermakov ${ }^{\dagger}$, Alireza Esteghamati ${ }^{\dagger}$, Emerito Jose A Faraon ${ }^{\dagger}$, Farshad Farzadfar ${ }^{\dagger}$, Seyed-Mohammad Fereshtehnejad ${ }^{\dagger}$, Daniel Obadare Fijabi ${ }^{\dagger}$, Mohammad H Forouzanfar ${ }^{\dagger}$, Urbano Fra. Paleo ${ }^{\dagger}$, Lynne Gaffikin ${ }^{\dagger}$, Amiran Gamkrelidze ${ }^{\dagger}$, Fortuné Gbètoho Gankpé ${ }^{\dagger}$, Johanna M Geleijnse ${ }^{\dagger}$, Bradford D Gessner ${ }^{\dagger}$, Katherine B Gibney ${ }^{\dagger}$, Ibrahim Abdelmageem Mohamed Ginawi ${ }^{\dagger}$, Elizabeth L Glaser ${ }^{\dagger}$, Philimon Gona ${ }^{\dagger}$, Atsushi Goto ${ }^{\dagger}$, Hebe N Gouda ${ }^{\dagger}$, Harish Chander Gugnani ${ }^{\dagger}$, Rajeev Gupta ${ }^{\dagger}$, Rahul Gupta ${ }^{\dagger}$, Nima Hafezi-Nejad ${ }^{\dagger}$, Randah Ribhi Hamadeh ${ }^{\dagger}$, Mouhanad Hammami ${ }^{\dagger}$, Graeme J Hankey ${ }^{\dagger}$, Hilda L Harb ${ }^{\dagger}$, Josep Maria Haro ${ }^{\dagger}$, Rasmus Havmoeller ${ }^{\dagger}$, Simon I Hay ${ }^{\dagger}$, Mohammad T Hedayati ${ }^{\dagger}$, Ileana B Heredia Pi ${ }^{\dagger}$, Hans W Hoek ${ }^{\dagger}$, John C Hornberger ${ }^{\dagger}, \mathrm{H}^{\text {Dean Hosgood }}{ }^{\dagger}$, Peter J Hotez $^{\dagger}$, Damian G Hoy ${ }^{\dagger}$, John J Huang ${ }^{\dagger}$, Kim M Iburg ${ }^{\dagger}$, Bulat T Idrisov ${ }^{\dagger}$, Kaire Innos $^{\dagger}$, Kathryn $\mathrm{H}$ Jacobsen ${ }^{\dagger}$, Panniyammakal Jeemon ${ }^{\dagger}$, Paul $\mathrm{N}$ Jensen ${ }^{\dagger}$, Vivekanand $\mathrm{Jha}^{\dagger}$, Guohong Jiang ${ }^{\dagger}$, Jost B Jonas ${ }^{\dagger}$, Knud Juel ${ }^{\dagger}$, Haidong Kan ${ }^{\dagger}$, Ida Kankindi $^{\dagger}$, Nadim E Karam ${ }^{\dagger}$, André Karch ${ }^{\dagger}$, Corine Kakizi Karema ${ }^{\dagger}$, Anil Kaul ${ }^{\dagger}$, Norito Kawakami ${ }^{\dagger}$, Dhruv S Kazi ${ }^{\dagger}$, Andrew $\mathrm{H} \mathrm{Kemp}^{\dagger}$, Andre Pascal Kengne ${ }^{\dagger}$, Andre Keren ${ }^{\dagger}$, Maia Kereselidze ${ }^{\dagger}$, Yousef Saleh Khader ${ }^{\dagger}$, Shams Eldin Ali Hassan Khalifa $^{\dagger}$, Ejaz Ahmed Khan ${ }^{\dagger}$, Young-Ho Khang ${ }^{\dagger}$, Irma Khonelidze ${ }^{\dagger}$, Yohannes Kinfu $^{\dagger}$, Jonas M Kinge ${ }^{\dagger}$, Luke Knibbs ${ }^{\dagger}$, Yoshihiro Kokubo ${ }^{\dagger}$, S Kosen ${ }^{\dagger}$, Barthelemy Kuate Defo ${ }^{\dagger}$, Veena S Kulkarni ${ }^{\dagger}$, Chanda Kulkarni ${ }^{\dagger}$, Kaushalendra Kumar ${ }^{\dagger}$, Ravi B Kumar $^{\dagger}$, G Anil Kumar ${ }^{\dagger}$, Gene F Kwan ${ }^{\dagger}$, Taavi Lai ${ }^{\dagger}$, Arjun Lakshmana Balaji ${ }^{\dagger}$, Hilton Lam $^{\dagger}$, Qing Lan ${ }^{\dagger}$, Van C Lansingh ${ }^{\dagger}$, Heidi J Larson ${ }^{\dagger}$, Anders Larsson ${ }^{\dagger}$, Jong-Tae Lee $^{\dagger}$, James Leigh ${ }^{\dagger}$, Mall Leinsalu ${ }^{\dagger}$, Ricky Leung ${ }^{\dagger}$, Yichong Li ${ }^{\dagger}$, Yongmei Li ${ }^{\dagger}$, Graça Maria Ferreira De Lima ${ }^{\dagger}$, Hsien-Ho Lin ${ }^{\dagger}$, Steven E Lipshultz ${ }^{\dagger}$, Shiwei Liư ${ }^{\dagger}$, Yang $\mathrm{Liu}^{\dagger}$, Belinda K Lloyd ${ }^{\dagger}$, Paulo A Lotufo ${ }^{\dagger}$, Vasco Manuel Pedro Machado ${ }^{\dagger}$, Jennifer $\mathrm{H}$ Maclachlan $^{\dagger}$, Carlos Magis-Rodriguez ${ }^{\dagger}$, Marek Majdan ${ }^{\dagger}$, Christopher Chabila Mapoma $^{\dagger}$, Wagner Marcenes ${ }^{\dagger}$, Melvin Barrientos Marzan ${ }^{\dagger}$, Joseph R Masci ${ }^{\dagger}$, Mohammad Taufiq Mashal ${ }^{\dagger}$, Amanda J Mason-Jones ${ }^{\dagger}$, Bongani M Mayosi ${ }^{\dagger}$, Tasara $\mathrm{T}$ Mazorodze ${ }^{\dagger}$, Abigail Cecilia Mckay ${ }^{\dagger}$, Peter A Meaney ${ }^{\dagger}$, Man Mohan Mehndiratta ${ }^{\dagger}$, Fabiola Mejia-Rodriguez ${ }^{\dagger}$, Yohannes Adama Melaku ${ }^{\dagger}$, Ziad A Memish ${ }^{\dagger}$, Walter Mendoza $^{\dagger}$, Ted R Miller ${ }^{\dagger}$, Edward J Mills ${ }^{\dagger}$, Karzan Abdulmuhsin Mohammad ${ }^{\dagger}$, Ali $\mathrm{H}$ Mokdad $^{\dagger}$, Glen Liddell Mola ${ }^{\dagger}$, Lorenzo Monasta ${ }^{\dagger}$, Marcella Montico ${ }^{\dagger}$, Ami R Moore ${ }^{\dagger}$, Rintaro Mori ${ }^{\dagger}$, Wilkister Nyaora Moturi ${ }^{\dagger}$, Mitsuru Mukaigawara ${ }^{\dagger}$, Kinnari S Murthy ${ }^{\dagger}$, Aliya Naheed ${ }^{\dagger}$, Kovin S Naidoo ${ }^{\dagger}$, Luigi Naldi ${ }^{\dagger}$, Vinay Nangia ${ }^{\dagger}, \mathrm{K} \mathrm{M}$ Venkat Narayan ${ }^{\dagger}$, Denis Nash ${ }^{\dagger}$, Chakib Nejjari ${ }^{\dagger}$, Robert G Nelson ${ }^{\dagger}$, Sudan Prasad Neupane ${ }^{\dagger}$, Charles R Newton ${ }^{\dagger}$, Marie $\mathrm{Ng}^{\dagger}$, Muhammad Imran Nisar ${ }^{\dagger}$, Sandra Nolte $^{\dagger}$, Ole F Norheim ${ }^{\dagger}$, Vincent Nowaseb ${ }^{\dagger}$, Luke Nyakarahuka ${ }^{\dagger}$, In-Hwan $\mathrm{Oh}^{\dagger}$, Takayoshi Ohkubo $^{\dagger}$, Bolajoko O Olusanya ${ }^{\dagger}$, Saad B Omer ${ }^{\dagger}$, John Nelson Opio ${ }^{\dagger}$, Orish Ebere Orisakwe ${ }^{\dagger}$, Jeyaraj D Pandian ${ }^{\dagger}$, Christina Papachristou ${ }^{\dagger}$, Angel J Paternina Caicedo ${ }^{\dagger}$, Scott B Patten ${ }^{\dagger}$, Vinod K Paul ${ }^{\dagger}$, Boris Igor Pavlin ${ }^{\dagger}$, Neil Pearce $^{\dagger}$, David M Pereira ${ }^{\dagger}$, Aslam Pervaiz ${ }^{\dagger}$, Konrad Pesudovs ${ }^{\dagger}$, Max Petzold ${ }^{\dagger}$, Farshad Pourmalek ${ }^{\dagger}$, Dima Qato ${ }^{\dagger}$, Amado D Quezada ${ }^{\dagger}$, D Alex Quistberg ${ }^{\dagger}$, Anwar Rafay $^{\dagger}$, Kazem Rahimi ${ }^{\dagger}$, Vafa Rahimi-Movaghar ${ }^{\dagger}$, Sajjad Ur Rahman ${ }^{\dagger}$, Murugesan 
Raju†, Saleem M Rana ${ }^{\dagger}$, Homie Razavi ${ }^{\dagger}$, Robert Quentin Reilly ${ }^{\dagger}$, Giuseppe Remuzzi ${ }^{\dagger}$, Jan Hendrik Richardus ${ }^{\dagger}$, Luca Ronfani ${ }^{\dagger}$, Nobhojit Roy ${ }^{\dagger}$, Nsanzimana Sabin $^{\dagger}$, Mohammad Yahya Saeedi ${ }^{\dagger}$, Mohammad Ali Sahraian ${ }^{\dagger}$, Genesis May J Samonte ${ }^{\dagger}$, Monika Sawhney ${ }^{\dagger}$, Ione J C Schneider ${ }^{\dagger}$, David C Schwebel ${ }^{\dagger}$, Soraya Seedat $^{\dagger}$, Sadaf G Sepanlou ${ }^{\dagger}$, Edson E Servan-Mori ${ }^{\dagger}$, Sara Sheikhbahaei ${ }^{\dagger}$, Kenji Shibuya ${ }^{\dagger}$, Hwashin Hyun Shin ${ }^{\dagger}$, Ivy Shiue ${ }^{\dagger}$, Rupak Shivakoti ${ }^{\dagger}$, Inga Dora Sigfusdottir ${ }^{\dagger}$, Donald H Silberberg ${ }^{\dagger}$, Andrea P Silva ${ }^{\dagger}$, Edgar $\mathrm{P}$ Simard ${ }^{\dagger}$, Jasvinder A Singh $^{\dagger}$, Vegard Skirbekk ${ }^{\dagger}$, Karen Sliwa ${ }^{\dagger}$, Samir Soneji ${ }^{\dagger}$, Sergey S Soshnikov ${ }^{\dagger}$, Chandrashekhar T Sreeramareddy ${ }^{\dagger}$, Vasiliki Kalliopi Stathopoulou ${ }^{\dagger}, K_{\text {Konstantinos }}$ Stroumpoulis ${ }^{\dagger}$, Soumya Swaminathan ${ }^{\dagger}$, Bryan L Sykes ${ }^{\dagger}$, Karen M Tabb ${ }^{\dagger}$, Roberto Tchio Talongwa $^{\dagger}$, Eric Yeboah Tenkorang ${ }^{\dagger}$, Abdullah Sulieman Terkawi ${ }^{\dagger}$, Alan J Thomson $^{\dagger}$, Andrew L Thorne-Lyman ${ }^{\dagger}$, Jeffrey A Towbin ${ }^{\dagger}$, Jefferson Traebert ${ }^{\dagger}$, Bach $X \operatorname{Tran}^{\dagger}$, Zacharie Tsala Dimbuene ${ }^{\dagger}$, Miltiadis Tsilimbaris ${ }^{\dagger}$, Uche S Uchendu ${ }^{\dagger}$, Kingsley N Ukwaja ${ }^{\dagger}$, Selen Begüm Uzun ${ }^{\dagger}$, Andrew J Vallely ${ }^{\dagger}$, Tommi J Vasankari ${ }^{\dagger}$, $\mathrm{N}$ Venketasubramanian ${ }^{\dagger}$, Francesco S Violante ${ }^{\dagger}$, Vasiliy Victorovich Vlassov ${ }^{\dagger}$, Stein Emil Vollset ${ }^{\dagger}$, Stephen Waller ${ }^{\dagger}$, Mitchell T Wallin ${ }^{\dagger}$, Linhong Wang ${ }^{\dagger}$, XiaoRong Wang $^{\dagger}$, Yanping Wang ${ }^{\dagger}$, Scott Weichenthal ${ }^{\dagger}$, Elisabete Weiderpass ${ }^{\dagger}$, Robert G Weintraub $^{\dagger}$, Ronny Westerman ${ }^{\dagger}$, Richard A White ${ }^{\dagger}$, James D Wilkinson ${ }^{\dagger}$, Thomas Neil Williams ${ }^{\dagger}$, Solomon Meseret Woldeyohannes ${ }^{\dagger}$, John Q Wong ${ }^{\dagger}$, Gelin Xu ${ }^{\dagger}$, Yang C Yang ${ }^{\dagger}$, Yuichiro Yano ${ }^{\dagger}$, Gokalp Kadri Yentur ${ }^{\dagger}$, Paul Yip ${ }^{\dagger}$, Naohiro Yonemoto ${ }^{\dagger}$, Seok-Jun Yoon ${ }^{\dagger}$, Mustafa Younis ${ }^{\dagger}$, Chuanhua Yu ${ }^{\dagger}, \mathrm{Kim}_{\text {Yun }} \mathrm{Jin}^{\dagger}$, Maysaa El Sayed Zaki ${ }^{\dagger}$, Yong Zhao ${ }^{\dagger}$, Yingfeng Zheng ${ }^{\dagger}$, Maigeng Zhou ${ }^{\dagger}$, Jun Zhu ${ }^{\dagger}$, Xiao Nong Zou ${ }^{\dagger}$, Alan D Lopez $z^{\ddagger}$, and Theo Vos ${ }^{\ddagger}$

Institute for Health Metrics and Evaluation (Prof $C J L$ Murray DPhil, K F Ortblad MPH, C Guinovart PhD, S S Lim PhD, T M Wolock BA, D A Roberts BS, E A Dansereau BA, N Graetz BS, R M Barber BS, J C Brown MAIS, H Wang PhD, H C Duber MD, M Naghavi PhD, D Dicker BS, Prof L Dandona MD, K R Heuton BS, D E Phillips BS, T D Fleming BS, A D Flaxman PhD, B K Phillips BA, E K Johnson BA, M S Coggeshall BA, M H Forouzanfar MD, Prof A H Mokdad PhD, M Ng PhD, Prof T Vos PhD), Harborview and University of Washington Medicine, Seattle, WA, USA (Prof R G Ellenbogen MD), University of Washington, Seattle, WA, USA ( $R$ Alfonso-Cristancho PhD, Prof B O Anderson MD, P N Jensen MPH, D A Quistberg $\mathrm{PhD}$ ); Public Health Foundation of India, New Delhi, India (Prof L Dandona, $R$ Dandona PhD, G A Kumar PhD, K S Murthy MPH); Imperial College London, London, UK (K Foreman MPH, Prof T N Williams MD); Harvard School of Public Health (E L Ding ScD), Harvard University, Cambridge, MA, USA (Prof J A Salomon PhD); Faculty of Medicine, Cairo-University, Cairo, Egypt (Prof F AbdAllah MD); College of Health Sciences, School of Public Health (S F Abera MSc), Mekelle University, Mekelle, Ethiopia (Y A Melaku MPH); University of Texas School of Medicine San Antonio, San Antonio, TX, USA (J P Abraham MD); University College London, London, UK (Prof I Abubakar PhD); Weill Cornell Medical College in Qatar, Doha, Qatar ( $\mathrm{J}$ Abu-Raddad PhD); Institute of Community and Public Health-Birzeti University, Ramallah, West Bank, Occupied Palestinian Territory (N M Abu-Rmeileh PhD); Ministry of Health, Gaborone, Botswana (T Achoki MD); Evidence For Action (MamaYe), Freetown, Western 
Area, Sierra Leone (A O Adeyemo MHM); Association Ivoirienne Pour Le Bien Etre Familial, Abidjan, Côte d'Ivoire (A K Adou MD); University of Extremadura, Cáceres, Spain (Prof J C Adsuar PhD, U Fra.Paleo PhD); Institution of Public Health Sciences, Stockholm, Sweden (E E Agardh PhD); Makerere University, Kampala, Uganda (D Akena PhD, L Nyakarahuka MPH); Ministry of Health, Muscat, Oman (M J Al Kahbouri PhD, D Alasfoor MSc); Independent, Damascus, Syria (M I Albittar MSc); Grupo de Investigación en Ciencias de la Salud y Neurociencias (CISNEURO), Cartagena de Indias, Colombia (G Alcalá-Cerra MD); Facultad de Medicina, Departamento de Medicina Preventiva y Social, Universidad de la República, Montevideo, Uruguay (M A Alegretti MD); Debre Markos University, Debre Markos, Ethiopia (Z A Alemu MPH); National Guard Health Affairs, Riyadh, Saudi Arabia (S Alhabib PhD); University of Oxford, Oxford, UK (R Ali MSc, Prof S I Hay DPhil, K Rahimi DM); School of Public Health, University of Lorraine, Nancy, France (Prof F Alla PhD); Ministry of Health, Belmopan, Cayo, Belize (P J Allen MPH); Charité Universitätsmedizin Berlin, Berlin, Germany (U Alsharif DMD, S Nolte PhD, C Papachristou PhD); Government, Madrid, Spain (E Alvarez PhD); Universidad de Cartagena, Cartagena de Indias, Colombia (Prof N Alvis-Guzman PhD, A J Paternina Caicedo MSc); Albany State University, Albany, GA, USA (Prof A A Amankwaa PhD); Department of Epidemiology, University of Groningen, Groningen, The Netherlands and College of Medicine and Health Sciences, Bahir Dar University, Bahir Dar, Ethiopia (A T Amare MPH); Kurdistan Environmental Health Research Center, Kurdistan University of Medical Sciences, Sanandaj, Kurdistan, Iran (H Amini MSPH); Ministry of Public Health, Beirut, Lebanon(Prof W Ammar PhD, H L Harb MPH); College of Public Health, University of the Philippines Manila, Manila, Philippines (C A T Antonio MD, E J A Faraon MD); UNFPA, Kabul, Afghanistan (P Anwari MSc); Uppsala University, Uppsala, Sweden (J Ärnlöv PhD, Prof A Larsson PhD); Malaria \& Other Parasitic Diseases Division (C K Karema MSc), Ministry of Health, Kigali, Rwanda (A Binagwaho MMed [Ped], I Kankindi MSc); Institute of Microbiology and Immunology, School of Medicine, University of Belgrade, Belgrade, Serbia (Prof V S Arsic Arsenijevic PhD); Median Inc, Windsor, ON, Canada (A Artaman PhD); South Asian Public Health Forum, Islamabad, Pakistan (R J Asghar MD); Mashhad University of Medical Sciences, Mashhad, Iran (R Assadi PhD); Ministry of Health, Wellness, Human Services and Gender Relations, Castries, St. Lucia (L S Atkins $\mathrm{MPH}$ ); Public Health Agency of Canada, Toronto, ON, Canada (A Badawi PhD); Sri Ramachandra University, Chennai, India (Prof K Balakrishnan PhD); University of Birmingham, Birmingham, UK (A Banerjee DPhil); Stanford University School of Medicine (L Gaffikin DrPH), Stanford University (S Basu PhD), Stanford, CA, USA; Oxford University, Ho Chi Minh City, Vietnam (J Beardsley $\mathrm{MBChB}$ ); Madawalabu University, Bale Goba, Ethiopia (T Bekele MPH); Yale University, New Haven, CT, USA (Prof M L Bell PhD, J J Huang MD); King's College London, London, UK (E Bernabe PhD); Addis Ababa University, Addis Ababa, Ethiopia (T J Beyene MSC, K Deribe MPH); Wellington Hospital, Wellington, New Zealand (N Bhala DPhil); Postgraduate Institute of Medical Education and Research, Chandigarh, India (A Bhalla MD, Prof V Jha DM); Aga 
Khan University Medical Center, Nairobi, Kenya (Prof Z A Bhutta PhD); University of Missouri-Kansas City, Kansas City, MO, USA (A Bin Abdulhak MD); General Practice and Primary Health Care Academic Centre (P P Chiang PhD), University of Melbourne, Melbourne, VIC, Australia (J D Blore PhD, A Lakshmana Balaji MBBS, Prof A D Lopez PhD, R G Weintraub MB); Murdoch Children's Research Institute, Royal Children's Hospital, Melbourne, VIC, Australia (R G Weintraub); General Directorate of Health Research (B Bora Basara PhD, G K Yentur PhD), Ministry of Health, Ankara, Turkey (Prof U Dilmen MD, S B Uzan MD); World Bank, Washington, DC, USA (D Bose PhD); Danube-University Krems, Krems, Austria (Prof M Brainin PhD); University of Arizona, Tucson, AZ, USA (Prof N Breitborde PhD); Colombian National Health Observatory, Instituto Nacional de Salud, Bogota, Colombia (C A Castañeda-Orjuela MSc); Division of Pharmacoepidemiology and Pharmacovigilance, Spanish Medicines and Healthcare Products Agency (AEMPS), Ministry of Health, Madrid, Spain (F Catalá-López $\mathrm{PhD}$ ); National Tuberculosis Institute, Bangalore, India (V K Chadha MD); Institute of Epidemiology and Preventive Medicine (H Lin ScD), National Taiwan University, Taipei, Taiwan (Prof J Chang PhD); Department of Parasitology, College of Medicine, Taipei Medical University, Taipei, Taiwan and Center for International Tropical Medicine, College of Medicine, Taipei Medical University, Taipei, Taiwan (Prof T Chuang PhD); UNICEM, Montevideo, Uruguay (M Colomar MSc); Mayo Clinic, Rochester, MN, USA (Prof L T Cooper MD); MRC Lifecourse Epidemiology Unit, University of Southampton, Southampton, UK (Prof C Cooper FMedSci); Hospital Dr. Gustavo N. Collado, Chitre, Herrera, Panama (K J Courville $\mathrm{MD}$ ); Victorian Infectious Diseases Reference Laboratory, North Melbourne, VIC, Australia (B C Cowie PhD, J H Maclachlan MSc); University of California, San Diego, La Jolla, CA, USA (Prof M H Criqui MD); Emory University School of Medicine (A Dayama MD), Emory University, Atlanta, GA, USA (Prof Y Liu PhD, Prof K M V Narayan MD, S B Omer PhD); Griffith University, Brisbane, QLD, Australia (Prof D De Leo DSc); The Kirby Institute (A J Vallely PhD), University of New South Wales, Sydney, NSW, Australia (Prof L Degenhardt PhD); University of Auckland, Auckland, New Zealand (B Del Pozo-Cruz PhD); Beth Israel Medical Center, New York, NY, USA (Prof D C Des Jarlais PhD); Africa Medical and Research Foundation in Ethiopia, Addis Ababa, Ethiopia (M Dessalegn MPH); University of Peradeniya, Peradeniya, Sri Lanka (S D Dharmaratne MD); Sydney School of Public Health (T R Driscoll PhD), University of Sydney, Sydney, NSW, Australia (J Leigh PhD); National Institutes of Health, Montgomery Village, MD, USA (A M Durrani MD); The Institute of Social and Economic Studies of Population at the Russian Academy of Sciences, Moscow, Russia (Prof S P Ermakov DSc); Non-Communicable Diseases Research Center (F Farzadfar MD), Endocrinology and Metabolism Research Center (Prof A Esteghamati MD, N Hafezi-Nejad MD, S Sheikhbahaei MD), Sina Trauma and Surgery Research Center (Prof V Rahimi-Movaghar MD),MS Research Center (M A Sahraian MD), Digestive Diseases Research Institute (S G Sepanlou MD), Tehran University of Medical Sciences, Tehran, Iran; Department of Neurobiology, Care Sciences and Society (NVS) (S Fereshtehnejad MD), Department of Medical Epidemiology and 
Biostatistics (Prof E Weiderpass PhD),Karolinska Institutet, Stockholm, Sweden (R Havmoeller PhD); Heller Graduate School, (D O Fijabi MBBS), Heller School for Social Policy and Management (E L Glaser MSc), Brandeis University, Waltham, MA, USA (B T Idrisov MD); National Centre for Disease Control and Public Health, Tbilisi, Georgia (A Gamkrelidze PhD, M Kereselidze PhD, I Khonelidze MPA); Clinique Coopérative de Parakou, Parakou, Borgou, Benin (F G Gankpé MD); Division of Human Nutrition, Wageningen University, Wageningen, Netherlands (J M Geleijnse PhD); Agence de Medecine Preventive, Paris, France (B D Gessner MD); Eastern Health Clinical School (B K Lloyd PhD), Monash University, Melbourne, VIC, Australia (K B Gibney MBBS); Turning Point, Eastern Health, Fitzroy, VIC, Australia (B K Lloyd); College of Medicine, University of Hail, Hail, Saudi Arabia (I A M Ginawi MD); University of Massachusetts Medical School, Worcester, MA, USA (Prof P Gona PhD); Department of Diabetes Research, National Center for Global Health and Medicine, Tokyo, Japan (A Goto PhD); School of Population Health (D G Hoy PhD), University of Queensland, Brisbane, QLD, Australia (H N Gouda PhD, L Knibbs PhD); Public Health Division, Secretariat of the Pacific Community, Noumea, New Caledonia (D G Hoy); Saint James School of Medicine, Kralendijk, Bonaire, Netherlands Antilles (Prof H C Gugnani PhD); Fortis Escorts Hospital, Jaipur, India (R Gupta PhD); Kanawha Charleston Health Department, Charleston, WV, USA (R Gupta MD); Arabian Gulf University, Manama, Bahrain (Prof R R Hamadeh DPhil); Wayne County Department of Health and Human Services, Detroit, MI, USA (M Hammami MD); School of Medicine and Pharmacology, University of Western Australia, Perth, WA, Australia (Prof G J Hankey MD); Parc Sanitari Sant Joan de Déu, CIBERSAM, University of Barcelona, Sant Boi de Llobregat, Barcelona, Spain (Prof J M Haro MD); Mazandaran University of Medical Sciences, Sari, Mazandaran, Iran (Prof M T Hedayati PhD); National Institute of Public Health of Mexico, Cuernavaca, Morelos, Mexico (I B Heredia Pi PhD, F Mejia-Rodriguez MD, A D Quezada MSc, Prof E E Servan-Mori MSc); Parnassia Psychiatric Institute, The Hague, Netherlands (Prof H W Hoek MD); Cedar Associates, Menlo Park, CA, USA (Prof J C Hornberger MD); Albert Einstein College of Medicine, Bronx, NY, USA (Prof H D Hosgood PhD); Baylor College of Medicine, Houston, TX, USA (Prof P J Hotez PhD); Aarhus University, Aarhus, Denmark (K M Iburg PhD); National Institute for Health Development, Tallinn, Estonia (K Innos PhD, M Leinsalu PhD); George Mason University, Fairfax, VA, USA ( $K$ H Jacobsen PhD); Centre for Chronic Disease Control, New Delhi, India ( $P$ Jeemon PhD); Tianjin Centers for Diseases Control and Prevention, Tianjin, China (Prof G Jiang MD); Department of Ophthalmology, Medical Faculty Mannheim of the University of Heidelberg, Mannheim, Germany (Prof J B Jonas MD); The National Institute of Public Health, Copenhagen, Denmark (Prof K Juel PhD); Fudan University, Shanghai, China (Prof H Kan MD); University of Balamand, Beirut, Lebanon (Prof N E Karam MD); Helmholtz Centre for Infection Research, Braunschweig, Germany and German Center for Infection Research (DZIF), Hannover-Braunschweig site, Germany (A Karch MD); Oklahoma State University, Tulsa, OK, USA (A Kaul MD); School of Public Health (Prof N 
Kawakami MD), University of Tokyo, Tokyo, Japan (Prof K Shibuya MD); University of California San Francisco, San Francisco, CA, USA (D S Kazi MD); University of São Paulo, São Paulo, Brazil (Prof A H Kemp PhD, Prof P A Lotufo DrPH); South African Medical Research Council, Cape Town, Western Cape, South Africa (A P Kengne PhD); Cardiology, Hadassah Ein Kerem University Hospital, Jerusalem, Israel (Prof A Keren MD); Jordan University of Science and Technology, Al-Ramtha, Jordan (Prof Y S Khader ScD); Supreme Council of Health, Doha, Qatar (S E A H Khalifa MSc); Health Services Academy, Islamabad, Punjab, Pakistan (E A Khan MPH); Institute of Health Policy and Management, Seoul National University College of Medicine, Seoul, South Korea (Prof Y Khang PhD); University of Canberra, Canberra, ACT, Australia (Y Kinfu PhD); Department of Infectious Disease Epidemiology, Division of Infectious Disease Control and Department of Health Statistics, Division of Epidemiology (R A White PhD), Norwegian Institute of Public Health, Oslo, Norway (J M Kinge PhD, Prof V Skirbekk $\mathrm{PhD}$, Prof S E Vollset MD); Department of Preventive Cardiology, Department of Preventive Medicine and Epidemiologic Informatics, National Cerebral and Cardiovascular Center, Suita, Osaka, Japan (Y Kokubo PhD); Center for Community Empowerment, Health Policy \& Humanities, NIHRD, Jakarta, Indonesia (S Kosen MD); University of Montreal, Montreal, QC, Canada (Prof B Kuate Defo $\mathrm{PhD}$ ); Arkansas State University, Jonesboro, AR, USA (V S Kulkarni PhD); Rajrajeshwari Medical College \& Hospital, Bangalore, Karnataka, India (Prof C Kulkarni PhD); International Institute for Population Sciences, Mumbai, Maharashtra, India (K Kumar MPS); Indian Institute of Public Health, Public Health Foundation of India, Gurgaon, Haryana, India (R B Kumar MD); Boston Medical Center, Boston, MA, USA (G F Kwan MD); Fourth View Consulting, Tallinn, Estonia (T Lai PhD); Institute of Health Policy and Development Studies, National Institutes of Health, Manila, Philippines (Prof H Lam PhD); National Cancer Institute, Rockville, MD, USA (Q Lan PhD); IAPB and Vision 2020 LA, Weston, FL, USA (V C Lansingh PhD); London School of Hygiene and Tropical Medicine, Bloomsbury, UK (H J Larson PhD, Prof N Pearce PhD); Korea University, Seoul, South Korea (Prof J Lee PhD, Prof S Yoon PhD); University at Albany, The State University of New York, Rensselaer, NY, USA (R Leung PhD); National Center for Chronic and Non-communicable Disease Control and Prevention, Chinese Center for Disease Control and Prevention, Beijing, China (Y Li MPH, S Liu PhD, Prof L Wang MD, Prof M Zhou PhD); Genentech, Inc, South San Francisco, CA, USA (Y Li PhD); Unidade Local de Saude de Matosinhos (G M Lima BSC), Northern Region Health Administration (V M P Machado MSc), Public Health Department, Porto, Portugal; Wayne State University, Miami, FL, USA (S E Lipshultz MD); Centro Nacional para la Prevención y el Control del VIH/SIDA, Mexico City, Mexico (C Magis-Rodriguez PhD); Department of Public Health, Faculty of Health Sciences and Social Work, Trnava University, Trnava, Slovakia (M Majdan PhD); University of Zambia, Lusaka, Zambia (C C Mapoma PhD); Queen Mary, University of London, London, UK (Prof W Marcenes PhD); University of the East Ramon Magsaysay Medical Center, Quezon City, Philippines (M B Marzan MSc); Elmhurst Hospital Center, Mount Sinai Services, Elmhurst, NY, USA (Prof J R Masci MD); 
Ministry of Public Health, Kabul, Afghanistan (M T Mashal PhD); University of York, York, UK (A J Mason-Jones PhD); Faculty of Health Sciences, Hatter Institute for Cardiovascular Research in Africa (Prof K Sliwa PhD), University of Cape Town, Cape Town, Western Cape, South Africa (Prof B M Mayosi DPhil); AIDC EC, Port Elizabeth, Eastern Cape, South Africa (T T Mazorodze MA); EmergentCorp, Belize City, Belize (A C Mckay PhD); Pereleman School of Medicine (P A Meaney MD), University of Pennsylvania, Philadelphia, PA, USA (Prof D H Silberberg MD); Janakpuri Superspecialty Hospital, New Delhi, India (Prof M M Mehndiratta MD); Ministry of Health, Riyadh, Saudi Arabia (Prof Z A Memish MD, M Y Saeedi PhD); UNFPA, Lima, Peru (W Mendoza MD); Pacific Institute for Research \& Evaluation, Calverton MD, USA and Centre for Population Health Research, Curtin University, Perth, WA, Australia, (T R Miller PhD); University of Ottawa, Ottawa, ON, Canada (E J Mills PhD); University of Salahaddin, Erbil, Iraq (K A Mohammad PhD); University of Papua New Guinea, Port Moresby, NCD, Papua New Guinea (Prof G L Mola MD); Institute for Maternal and Child Health-IRCCS "Burlo Garofolo," Trieste, Italy (L Monasta DSc, M Montico Msc, L Ronfani PhD); University of North Texas, Denton, TX, USA (Prof A R Moore PhD); National Center for Child Health and Development, Setagaya, Tokyo, Japan (R Mori PhD); Egerton University, Egerton, Rift Valley, Kenya (W N Moturi PhD); Tokyo Medical and Dental University, Bunkyo-ku, Tokyo, Japan (M Mukaigawara MD); International Centre for Diarrhoeal Diseases Research, Dhaka, Bangladesh (A Naheed PhD); University of KwaZulu-Natal, Durban, KwaZulu-Natal, South Africa (Prof K S Naidoo PhD); Azienda Ospedaliera papa Giovanni XXIII, Bergamo, Italy (Prof L Naldi MD); Suraj Eye Institute, Nagpur, Maharashtra, India (Prof V Nangia MD); School of Public Health, City University of New York, New York, NY, USA (Prof D Nash PhD); Faculty of Medicine, Fez, Morocco (Prof $\mathrm{C}$ Nejjari $\mathrm{PhD}$ ); National Institute of Diabetes and Digestive and Kidney Diseases, Phoenix, AZ, USA (R G Nelson PhD); Norwegian Center for Addiction Research (SERAF), University of Oslo, Oslo, Norway (S P Neupane MBBS); Kenya Medical Research Institute Wellcome Trust Programme, Kilifi, Kenya (Prof C R Newton MD); Aga Khan University, Karachi, Pakistan (M I Nisar MSc); University of Bergen, Bergen, Norway (Prof O F Norheim PhD, Prof S E Vollset); Polytechnic of Namibia, Windhoek, Namibia (V Nowaseb MSc); Kyung Hee University, Seoul, Korea (I Oh PhD); Teikyo University School of Medicine, Tokyo, Japan (Prof T Ohkubo MD); Center for Healthy Start Initiative, Ikoyi, Lagos, Nigeria (B O Olusanya $\mathrm{PhD}$ ); Lira District Local Government, Lira Municipal Council, Northern Uganda, Uganda (J N Opio MPH); Toxicology Unit, Faculty of Pharmacy, University of Port Harcourt, Port Harcourt, Rivers State, Nigeria (Prof O E Orisakwe PhD); Christian Medical College Ludhiana, Ludhiana, India (Prof J D Pandian MD); University of Calgary, Calgary, AB, Canada (Prof S B Patten PhD); AlIMS, New Delhi, India (Prof V K Paul MD); Independent, Waigani, NCD, Papua New Guinea (B I Pavlin $\mathrm{MD})$; 3B's Research Group-Biomaterials, Biodegradables and Biomimetics, University of Minho, Headquarters of the European Institute of Excellence on Tissue Engineering and Regenerative Medicine and ICVS/3B's-PT Government Associate Laboratory, Braga, Portugal (Prof D M Pereira PhD); Postgraduate Medical Institute, 
Lahore, Punjab, Pakistan (A Pervaiz MHA); Flinders University, Adelaide, SA, Australia (Prof K Pesudovs PhD); Centre for Applied Biostatistics, Sahlgrenska Academy, University of Gothenburg, Gothenburg, Sweden (Prof M Petzold PhD); University of British Columbia, Vancouver, BC, Canada (F Pourmalek PhD); College of Pharmacy, University of Illinois, Chicago, IL, USA (Prof D Qato PhD); Contech Intl., Lahore, Punjab, Pakistan (A Rafay MS); Hamad Medical Corporation, Doha, Qatar (S U Rahman FRCPCH); University of Missouri, Columbia, MO, USA (M Raju PhD); Department of Public Health, University of the Punjab, Lahore, Punjab, Pakistan (S M Rana PhD); Center for Disease Analysis, Louisville, CO, USA (H Razavi PhD); Private consultant, Cairns, QLD, Australia (R Q Reilly $\mathrm{MPH}$ ); IRCCS Mario Negri Institute for Pharmacological Research, Centro Anna Maria Astori, Bergamo, Italy (Prof G Remuzzi MD); Erasmus MC, University Medical Center Rotterdam, Rotterdam, Netherlands (Prof J H Richardus PhD); BARC Hospital, Mumbai, Maharashtra, India (Prof N Roy MD); Rwanda Bio-Medical Center, Kigali, Rwanda (N Sabin MD); National HIV/AIDS \& STI Surveillance and Strategic Information Unit, National Epidemiology Center, Department of Health, Manila, National Capital Region, Philippines (G M J Samonte MD); Marshall University, Huntington, WV, USA M Sawhney PhD); Federal University of Santa Catarina, Florianópolis, Brazil (I J C Schneider PhD); University of Alabama at Birmingham, Birmingham, AL, USA (Prof D C Schwebel PhD, J A Singh MD); Stellenbosch University, Cape Town, Western Cape, South Africa (Prof S Seedat $\mathrm{PhD}$ ); Health Canada, Ottawa, ON, Canada (H H Shin PhD, S Weichenthal PhD); Heriot-Watt University, Edinburgh, UK (I Shiue PhD); Center for Clinical Global Health Education (R Shivakoti PhD), Johns Hopkins Bloomberg School of Public Health, Johns Hopkins University, Baltimore, MD, USA (B X Tran PhD); Reykjavik University, Reykjavik, Iceland (Prof I D Sigfusdottir PhD); Instituto Nacional de Epidemiología Dr Juan H Jara, Mar del Plata, Buenos Aires, Argentina (A P Silva MgSc); Surveillance and Health Services Research Program American Cancer Society, Atlanta, GA, USA (E P Simard PhD); Dartmouth College, Lebanon, NH, USA (S Soneji PhD); Federal Research Institute for Health Organization and Informatics of Ministry of Health of the Russian Federation, Moscow, Russia (S S Soshnikov PhD); Faculty of Medicine and Health Sciences, University Tunku Abdul Rahman, Kajang, Selangor, Malaysia (C T Sreeramareddy MD); Centre Hospitalier Nord Deux-Sevres, Bressuire, France (V K Stathopoulou MD); KEELPNO (Centre for Disease Control, Greece, dispatched to "Alexandra" General Hospital of Athens), Athens, Greece (K Stroumpoulis PhD); National Institute for Research in Tuberculosis, Chennai, Tamil Nadu, India (S Swaminathan MD); Department of Criminology, Law and Society (and Sociology), University of California-Irvine, Chicago, IL, USA (B L Sykes PhD); University of Illinois, Champaign, IL, USA (K M Tabb PhD); Ministry of Health-MINSANTE, Yaounde, Centre, Cameroon (R T Talongwa MD); Memorial University, St John's, NL, Canada (E Y Tenkorang PhD); Department of Anesthesiology, University of Virginia, Charlottesville, VA, USA, and Department of Anesthesiology, King Fahad Medical City, Riyadh, Saudi Arabia (A S Terkawi MD); Adaptive Knowledge Management, Victoria, BC, Canada (A J Thomson PhD); The Earth Institute, Columbia University, New York, NY, USA 
(A L Thorne-Lyman ScD); Cincinnati Children's Hospital Medical Center, Cincinnati, $\mathrm{OH}$, USA (Prof J A Towbin MD); University of Southern Santa Catarina, Palhoça, Santa Catarina, Brazil (Prof J Traebert PhD); Department of Population Sciences and Development, Faculty of Economics and Management, University of Kinshasa, Kinshasa, Democratic Republic of the Congo (Z Tsala Dimbuene PhD); Department of Medicine, University of Crete, Heraklion, Greece (Prof M Tsilimbaris PhD); Department of Veterans Affairs, Washington, DC, USA (U S Uchendu MD); Department of Internal Medicine, Federal Teaching Hospital Abakaliki, Abakailiki, Ebonyi State, Nigeria (K N Ukwaja MD); UKK Institute for Health Promotion Research, Tampere, Finland (Prof T J Vasankari PhD); Neuroscience Centre, Raffles Hospital, Singapore, Singapore (N Venketasubramanian MD); University of Bologna, Bologna, Italy (Prof F S Violante MD); Higher School of Economics, Moscow, Russia (Prof V V Vlassov MD); Uniformed Services University of Health Sciences, Bethesda, MD, USA (S Waller MD); VA Medical Center and Georgetown University Neurology Department, Washington, DC, USA (M T Wallin MD); Shandong University Affiliated Jinan Central Hospital, Jinan, China (X R Wang PhD); National Office for Maternal and Child Health Surveillance, Chengdu, China (Prof Y Wang BS, Prof J Zhu MD); University of Marburg, Marburg, Germany (R Westerman PhD); University of Miami, Miami, FL, USA (J D Wilkinson MD); Institute of Public Health, University of Gondar, Gondar, Ethiopia (S M Woldeyohannes MPH); Ateneo School of Medicine and Public Health, Pasig City, Metro Manila, Philippines (J Q Wong MD); Nanjing University School of Medicine, Jinling Hospital, Nanjing, China (Prof G Xu PhD); University of North Carolina at Chapel Hill, Chapel Hill, NC, USA (Y C Yang PhD); Division of Cardiovascular Medicine, Jichi Medical University School of Medicine, Shimotsuke, Tochigi, Japan (Y Yano MD); The University of Hong Kong, Hong Kong, Hong Kong (Prof P Yip $\mathrm{PhD}$ ); National Center of Neurology and Psychiatry, Kodira, Tokyo, Japan (N Yonemoto MPH); Jackson State University, Jackson, MS, USA (Prof M Younis PhD); Department of Epidemiology and Biostatistics, School of Public Health and Global Health Institute, Wuhan University, Wuhan, China (Prof C Yu PhD); TCM MEDICAL TK SDN BHD, Nusajaya, Johor Bahru, Malaysia (K Yun Jin PhD); Mansoura Faculty of Medicine, Mansoura, Egypt (Prof M E S Zaki MD); Chongqing Medical University, Chongqing, China (Prof $Y$ Zhao MD); Zhongshan Ophthalmic Center, Sun Yat-sen University, Guangzhou, China (Y Zheng PhD); Cancer Institute/Hospital, Chinese Academy of Medical Sciences, Beijing, China (Prof X N Zou MD)

\section{Affiliations}

Institute for Health Metrics and Evaluation (Prof $C \mathrm{~J} L$ Murray DPhil, K F Ortblad MPH, C Guinovart PhD, S S Lim PhD, T M Wolock BA, D A Roberts BS, E A Dansereau BA, N Graetz BS, R M Barber BS, J C Brown MAIS, H Wang PhD, H C Duber MD, M Naghavi PhD, D Dicker BS, Prof L Dandona MD, K R Heuton BS, D E Phillips BS, T D Fleming BS, A D Flaxman PhD, B K Phillips BA, E K Johnson BA, M S Coggeshall BA, M H Forouzanfar MD, Prof A H Mokdad PhD, M Ng PhD, Prof T Vos PhD), Harborview and University of Washington Medicine, Seattle, WA, USA 
(Prof R G Ellenbogen MD), University of Washington, Seattle, WA, USA ( $R$ Alfonso-Cristancho PhD, Prof B O Anderson MD, P N Jensen MPH, D A Quistberg $\mathrm{PhD}$ ); Public Health Foundation of India, New Delhi, India (Prof L Dandona, $R$ Dandona PhD, G A Kumar PhD, K S Murthy MPH); Imperial College London, London, UK (K Foreman MPH, Prof T N Williams MD); Harvard School of Public Health (E L Ding ScD), Harvard University, Cambridge, MA, USA (Prof J A Salomon PhD); Faculty of Medicine, Cairo-University, Cairo, Egypt (Prof F AbdAllah MD); College of Health Sciences, School of Public Health (S F Abera MSc), Mekelle University, Mekelle, Ethiopia (Y A Melaku MPH); University of Texas School of Medicine San Antonio, San Antonio, TX, USA (J P Abraham MD); University College London, London, UK (Prof I Abubakar PhD); Weill Cornell Medical College in Qatar, Doha, Qatar ( $\mathrm{L} J$ Abu-Raddad PhD); Institute of Community and Public Health-Birzeti University, Ramallah, West Bank, Occupied Palestinian Territory (N M Abu-Rmeileh PhD); Ministry of Health, Gaborone, Botswana (T Achoki MD); Evidence For Action (MamaYe), Freetown, Western Area, Sierra Leone (A O Adeyemo MHM); Association Ivoirienne Pour Le Bien Etre Familial, Abidjan, Côte d'Ivoire (A K Adou MD); University of Extremadura, Cáceres, Spain (Prof J C Adsuar PhD, U Fra.Paleo PhD); Institution of Public Health Sciences, Stockholm, Sweden (E E Agardh PhD); Makerere University, Kampala, Uganda (D Akena PhD, L Nyakarahuka MPH); Ministry of Health, Muscat, Oman (M J Al Kahbouri PhD, D Alasfoor MSc); Independent, Damascus, Syria (M I Albittar MSc); Grupo de Investigación en Ciencias de la Salud y Neurociencias (CISNEURO), Cartagena de Indias, Colombia (G Alcalá-Cerra MD); Facultad de Medicina, Departamento de Medicina Preventiva y Social, Universidad de la República, Montevideo, Uruguay (M A Alegretti MD); Debre Markos University, Debre Markos, Ethiopia (Z A Alemu MPH); National Guard Health Affairs, Riyadh, Saudi Arabia (S Alhabib PhD); University of Oxford, Oxford, UK (R Ali MSc, Prof S I Hay DPhil, K Rahimi DM); School of Public Health, University of Lorraine, Nancy, France (Prof F Alla PhD); Ministry of Health, Belmopan, Cayo, Belize (P J Allen MPH); Charité Universitätsmedizin Berlin, Berlin, Germany (U Alsharif DMD, S Nolte PhD, C Papachristou PhD); Government, Madrid, Spain (E Alvarez PhD); Universidad de Cartagena, Cartagena de Indias, Colombia (Prof N Alvis-Guzman PhD, A J Paternina Caicedo MSc); Albany State University, Albany, GA, USA (Prof A A Amankwaa PhD); Department of Epidemiology, University of Groningen, Groningen, The Netherlands and College of Medicine and Health Sciences, Bahir Dar University, Bahir Dar, Ethiopia (A T Amare MPH); Kurdistan Environmental Health Research Center, Kurdistan University of Medical Sciences, Sanandaj, Kurdistan, Iran (H Amini MSPH); Ministry of Public Health, Beirut, Lebanon(Prof W Ammar PhD, H L Harb MPH); College of Public Health, University of the Philippines Manila, Manila, Philippines (C A T Antonio MD, E J A Faraon MD); UNFPA, Kabul, Afghanistan (P Anwari MSc); Uppsala University, Uppsala, Sweden (J Ärnlöv PhD, Prof A Larsson PhD); Malaria \& Other Parasitic Diseases Division (C K Karema MSc), Ministry of Health, Kigali, Rwanda (A Binagwaho MMed [Ped], I Kankindi MSc); Institute of Microbiology and Immunology, School of Medicine, University of Belgrade, Belgrade, Serbia (Prof V S Arsic 
Arsenijevic PhD); Median Inc, Windsor, ON, Canada (A Artaman PhD); South Asian Public Health Forum, Islamabad, Pakistan (R J Asghar MD); Mashhad University of Medical Sciences, Mashhad, Iran (R Assadi PhD); Ministry of Health, Wellness, Human Services and Gender Relations, Castries, St. Lucia (L S Atkins $\mathrm{MPH}$ ); Public Health Agency of Canada, Toronto, ON, Canada (A Badawi PhD); Sri Ramachandra University, Chennai, India (Prof K Balakrishnan $\mathrm{PhD}$ ); University of Birmingham, Birmingham, UK (A Banerjee DPhil); Stanford University School of Medicine (L Gaffikin DrPH), Stanford University (S Basu PhD), Stanford, CA, USA; Oxford University, Ho Chi Minh City, Vietnam (J Beardsley $\mathrm{MBChB}$ ); Madawalabu University, Bale Goba, Ethiopia (T Bekele MPH); Yale University, New Haven, CT, USA (Prof M L Bell PhD, J J Huang MD); King's College London, London, UK (E Bernabe PhD); Addis Ababa University, Addis Ababa, Ethiopia (T J Beyene MSC, K Deribe MPH); Wellington Hospital, Wellington, New Zealand (N Bhala DPhil); Postgraduate Institute of Medical Education and Research, Chandigarh, India (A Bhalla MD, Prof V Jha DM); Aga Khan University Medical Center, Nairobi, Kenya (Prof Z A Bhutta PhD); University of Missouri-Kansas City, Kansas City, MO, USA (A Bin Abdulhak MD); General Practice and Primary Health Care Academic Centre (P P Chiang PhD), University of Melbourne, Melbourne, VIC, Australia (J D Blore PhD, A Lakshmana Balaji MBBS, Prof A D Lopez PhD, R G Weintraub MB); Murdoch Children's Research Institute, Royal Children's Hospital, Melbourne, VIC, Australia (R G Weintraub); General Directorate of Health Research (B Bora Basara PhD, G K Yentur PhD), Ministry of Health, Ankara, Turkey (Prof U Dilmen MD, S B Uzan MD); World Bank, Washington, DC, USA (D Bose PhD); Danube-University Krems, Krems, Austria (Prof M Brainin PhD); University of Arizona, Tucson, AZ, USA (Prof N Breitborde PhD); Colombian National Health Observatory, Instituto Nacional de Salud, Bogota, Colombia (C A Castañeda-Orjuela MSc); Division of Pharmacoepidemiology and Pharmacovigilance, Spanish Medicines and Healthcare Products Agency (AEMPS), Ministry of Health, Madrid, Spain (F Catalá-López $\mathrm{PhD}$ ); National Tuberculosis Institute, Bangalore, India (V K Chadha MD); Institute of Epidemiology and Preventive Medicine (H Lin ScD), National Taiwan University, Taipei, Taiwan (Prof J Chang PhD); Department of Parasitology, College of Medicine, Taipei Medical University, Taipei, Taiwan and Center for International Tropical Medicine, College of Medicine, Taipei Medical University, Taipei, Taiwan (Prof T Chuang PhD); UNICEM, Montevideo, Uruguay (M Colomar MSc); Mayo Clinic, Rochester, MN, USA (Prof L T Cooper MD); MRC Lifecourse Epidemiology Unit, University of Southampton, Southampton, UK (Prof C Cooper FMedSci); Hospital Dr. Gustavo N. Collado, Chitre, Herrera, Panama (K J Courville $\mathrm{MD})$; Victorian Infectious Diseases Reference Laboratory, North Melbourne, VIC, Australia (B C Cowie PhD, J H Maclachlan MSc); University of California, San Diego, La Jolla, CA, USA (Prof M H Criqui MD); Emory University School of Medicine (A Dayama MD), Emory University, Atlanta, GA, USA (Prof Y Liu PhD, Prof K M V Narayan MD, S B Omer PhD); Griffith University, Brisbane, QLD, Australia (Prof D De Leo DSc); The Kirby Institute (A J Vallely PhD), University of New South Wales, Sydney, NSW, Australia (Prof L Degenhardt PhD); University of 
Auckland, Auckland, New Zealand (B Del Pozo-Cruz PhD); Beth Israel Medical Center, New York, NY, USA (Prof D C Des Jarlais PhD); Africa Medical and Research Foundation in Ethiopia, Addis Ababa, Ethiopia (M Dessalegn MPH); University of Peradeniya, Peradeniya, Sri Lanka (S D Dharmaratne MD); Sydney School of Public Health (T R Driscoll PhD), University of Sydney, Sydney, NSW, Australia (J Leigh PhD); National Institutes of Health, Montgomery Village, MD, USA (A M Durrani MD); The Institute of Social and Economic Studies of Population at the Russian Academy of Sciences, Moscow, Russia (Prof S P Ermakov DSc); Non-Communicable Diseases Research Center (F Farzadfar $M D$ ), Endocrinology and Metabolism Research Center (Prof A Esteghamati MD, N Hafezi-Nejad MD, S Sheikhbahaei MD), Sina Trauma and Surgery Research Center (Prof V Rahimi-Movaghar MD),MS Research Center (M A Sahraian MD), Digestive Diseases Research Institute (S G Sepanlou MD), Tehran University of Medical Sciences, Tehran, Iran; Department of Neurobiology, Care Sciences and Society (NVS) (S Fereshtehnejad MD), Department of Medical Epidemiology and Biostatistics (Prof E Weiderpass PhD),Karolinska Institutet, Stockholm, Sweden (R Havmoeller PhD); Heller Graduate School, (D O Fijabi MBBS), Heller School for Social Policy and Management ( $L$ L Glaser MSc), Brandeis University, Waltham, MA, USA (B T Idrisov MD); National Centre for Disease Control and Public Health, Tbilisi, Georgia (A Gamkrelidze PhD, M Kereselidze PhD, I Khonelidze MPA); Clinique Coopérative de Parakou, Parakou, Borgou, Benin (F G Gankpé MD); Division of Human Nutrition, Wageningen University, Wageningen, Netherlands (J M Geleijnse PhD); Agence de Medecine Preventive, Paris, France (B D Gessner MD); Eastern Health Clinical School (B K Lloyd PhD), Monash University, Melbourne, VIC, Australia (K B Gibney MBBS); Turning Point, Eastern Health, Fitzroy, VIC, Australia (B K Lloyd); College of Medicine, University of Hail, Hail, Saudi Arabia (I A M Ginawi MD); University of Massachusetts Medical School, Worcester, MA, USA (Prof P Gona PhD); Department of Diabetes Research, National Center for Global Health and Medicine, Tokyo, Japan (A Goto PhD); School of Population Health (D G Hoy PhD), University of Queensland, Brisbane, QLD, Australia (H N Gouda PhD, L Knibbs $\mathrm{PhD}$ ); Public Health Division, Secretariat of the Pacific Community, Noumea, New Caledonia (D G Hoy); Saint James School of Medicine, Kralendijk, Bonaire, Netherlands Antilles (Prof H C Gugnani PhD); Fortis Escorts Hospital, Jaipur, India (R Gupta PhD); Kanawha Charleston Health Department, Charleston, WV, USA (R Gupta MD); Arabian Gulf University, Manama, Bahrain (Prof R R Hamadeh DPhil); Wayne County Department of Health and Human Services, Detroit, MI, USA (M Hammami MD); School of Medicine and Pharmacology, University of Western Australia, Perth, WA, Australia (Prof G J Hankey MD); Parc Sanitari Sant Joan de Déu, CIBERSAM, University of Barcelona, Sant Boi de Llobregat, Barcelona, Spain (Prof J M Haro MD); Mazandaran University of Medical Sciences, Sari, Mazandaran, Iran (Prof M T Hedayati PhD); National Institute of Public Health of Mexico, Cuernavaca, Morelos, Mexico (I B Heredia Pi PhD, F Mejia-Rodriguez MD, A D Quezada MSc, Prof E E Servan-Mori MSc); Parnassia Psychiatric Institute, The Hague, Netherlands (Prof H W Hoek MD); Cedar 
Associates, Menlo Park, CA, USA (Prof J C Hornberger MD); Albert Einstein College of Medicine, Bronx, NY, USA (Prof H D Hosgood PhD); Baylor College of Medicine, Houston, TX, USA (Prof P J Hotez PhD); Aarhus University, Aarhus, Denmark (K M lburg PhD); National Institute for Health Development, Tallinn, Estonia (K Innos PhD, M Leinsalu PhD); George Mason University, Fairfax, VA, USA ( $K$ H Jacobsen PhD); Centre for Chronic Disease Control, New Delhi, India ( $P$ Jeemon PhD); Tianjin Centers for Diseases Control and Prevention, Tianjin, China (Prof G Jiang MD); Department of Ophthalmology, Medical Faculty Mannheim of the University of Heidelberg, Mannheim, Germany (Prof J B Jonas MD); The National Institute of Public Health, Copenhagen, Denmark (Prof K Juel PhD); Fudan University, Shanghai, China (Prof H Kan MD); University of Balamand, Beirut, Lebanon (Prof N E Karam MD); Helmholtz Centre for Infection Research, Braunschweig, Germany and German Center for Infection Research (DZIF), Hannover-Braunschweig site, Germany (A Karch MD); Oklahoma State University, Tulsa, OK, USA (A Kaul MD); School of Public Health (Prof N Kawakami MD), University of Tokyo, Tokyo, Japan (Prof K Shibuya MD); University of California San Francisco, San Francisco, CA, USA (D S Kazi MD); University of São Paulo, São Paulo, Brazil (Prof A H Kemp PhD, Prof P A Lotufo DrPH); South African Medical Research Council, Cape Town, Western Cape, South Africa (A P Kengne PhD); Cardiology, Hadassah Ein Kerem University Hospital, Jerusalem, Israel (Prof A Keren MD); Jordan University of Science and Technology, Al-Ramtha, Jordan (Prof Y S Khader ScD); Supreme Council of Health, Doha, Qatar (S E A H Khalifa MSc); Health Services Academy, Islamabad, Punjab, Pakistan (E A Khan MPH); Institute of Health Policy and Management, Seoul National University College of Medicine, Seoul, South Korea (Prof Y Khang PhD); University of Canberra, Canberra, ACT, Australia (Y Kinfu PhD); Department of Infectious Disease Epidemiology, Division of Infectious Disease Control and Department of Health Statistics, Division of Epidemiology (R A White PhD), Norwegian Institute of Public Health, Oslo, Norway (J M Kinge PhD, Prof V Skirbekk PhD, Prof S E Vollset MD); Department of Preventive Cardiology, Department of Preventive Medicine and Epidemiologic Informatics, National Cerebral and Cardiovascular Center, Suita, Osaka, Japan (Y Kokubo PhD); Center for Community Empowerment, Health Policy \& Humanities, NIHRD, Jakarta, Indonesia (S Kosen MD); University of Montreal, Montreal, QC, Canada (Prof B Kuate Defo $\mathrm{PhD}$ ); Arkansas State University, Jonesboro, AR, USA (V S Kulkarni PhD); Rajrajeshwari Medical College \& Hospital, Bangalore, Karnataka, India (Prof C Kulkarni PhD); International Institute for Population Sciences, Mumbai, Maharashtra, India (K Kumar MPS); Indian Institute of Public Health, Public Health Foundation of India, Gurgaon, Haryana, India (R B Kumar MD); Boston Medical Center, Boston, MA, USA (G F Kwan MD); Fourth View Consulting, Tallinn, Estonia (T Lai PhD); Institute of Health Policy and Development Studies, National Institutes of Health, Manila, Philippines (Prof H Lam PhD); National Cancer Institute, Rockville, MD, USA (Q Lan PhD); IAPB and Vision 2020 LA, Weston, FL, USA (V C Lansingh PhD); London School of Hygiene and Tropical Medicine, Bloomsbury, UK (H J Larson PhD, Prof N Pearce PhD); Korea University, Seoul, 
South Korea (Prof J Lee PhD, Prof S Yoon PhD); University at Albany, The State University of New York, Rensselaer, NY, USA (R Leung PhD); National Center for Chronic and Non-communicable Disease Control and Prevention, Chinese Center for Disease Control and Prevention, Beijing, China (Y Li MPH, S Liu PhD, Prof L Wang MD, Prof M Zhou PhD); Genentech, Inc, South San Francisco, CA, USA (Y Li PhD); Unidade Local de Saude de Matosinhos (G M Lima BSC), Northern Region Health Administration (V M P Machado MSc), Public Health Department, Porto, Portugal; Wayne State University, Miami, FL, USA (S E Lipshultz MD); Centro Nacional para la Prevención y el Control del VIH/SIDA, Mexico City, Mexico (C Magis-Rodriguez PhD); Department of Public Health, Faculty of Health Sciences and Social Work, Trnava University, Trnava, Slovakia (M Majdan PhD); University of Zambia, Lusaka, Zambia (C C Mapoma PhD); Queen Mary, University of London, London, UK (Prof W Marcenes PhD); University of the East Ramon Magsaysay Medical Center, Quezon City, Philippines (M B Marzan MSc); Elmhurst Hospital Center, Mount Sinai Services, Elmhurst, NY, USA (Prof J R Masci MD); Ministry of Public Health, Kabul, Afghanistan (M T Mashal PhD); University of York, York, UK (A J Mason-Jones PhD); Faculty of Health Sciences, Hatter Institute for Cardiovascular Research in Africa (Prof K Sliwa PhD), University of Cape Town, Cape Town, Western Cape, South Africa (Prof B M Mayosi DPhil); AIDC EC, Port Elizabeth, Eastern Cape, South Africa (T T Mazorodze MA); EmergentCorp, Belize City, Belize (A C Mckay PhD); Pereleman School of Medicine (P A Meaney MD), University of Pennsylvania, Philadelphia, PA, USA (Prof D H Silberberg MD); Janakpuri Superspecialty Hospital, New Delhi, India (Prof M M Mehndiratta MD); Ministry of Health, Riyadh, Saudi Arabia (Prof Z A Memish MD, M Y Saeedi PhD); UNFPA, Lima, Peru (W Mendoza MD); Pacific Institute for Research \& Evaluation, Calverton MD, USA and Centre for Population Health Research, Curtin University, Perth, WA, Australia, (T R Miller PhD); University of Ottawa, Ottawa, ON, Canada (E J Mills PhD); University of Salahaddin, Erbil, Iraq (K A Mohammad PhD); University of Papua New Guinea, Port Moresby, NCD, Papua New Guinea (Prof G L Mola MD); Institute for Maternal and Child Health-IRCCS "Burlo Garofolo," Trieste, Italy (L Monasta DSc, M Montico Msc, L Ronfani PhD); University of North Texas, Denton, TX, USA (Prof A R Moore PhD); National Center for Child Health and Development, Setagaya, Tokyo, Japan (R Mori PhD); Egerton University, Egerton, Rift Valley, Kenya (W N Moturi PhD); Tokyo Medical and Dental University, Bunkyo-ku, Tokyo, Japan (M Mukaigawara MD); International Centre for Diarrhoeal Diseases Research, Dhaka, Bangladesh (A Naheed PhD); University of KwaZulu-Natal, Durban, KwaZulu-Natal, South Africa (Prof K S Naidoo PhD); Azienda Ospedaliera papa Giovanni XXIII, Bergamo, Italy (Prof L Naldi MD); Suraj Eye Institute, Nagpur, Maharashtra, India (Prof V Nangia MD); School of Public Health, City University of New York, New York, NY, USA (Prof D Nash PhD); Faculty of Medicine, Fez, Morocco (Prof C Nejjari PhD); National Institute of Diabetes and Digestive and Kidney Diseases, Phoenix, AZ, USA (R G Nelson PhD); Norwegian Center for Addiction Research (SERAF), University of Oslo, Oslo, Norway (S P Neupane MBBS); Kenya Medical Research Institute Wellcome Trust Programme, Kilifi, Kenya (Prof C R Newton MD); 
Aga Khan University, Karachi, Pakistan (M I Nisar MSc); University of Bergen, Bergen, Norway (Prof O F Norheim PhD, Prof S E Vollset); Polytechnic of Namibia, Windhoek, Namibia (V Nowaseb MSc); Kyung Hee University, Seoul, Korea (I Oh PhD); Teikyo University School of Medicine, Tokyo, Japan (Prof T Ohkubo MD); Center for Healthy Start Initiative, Ikoyi, Lagos, Nigeria (B O Olusanya $\mathrm{PhD}$ ); Lira District Local Government, Lira Municipal Council, Northern Uganda, Uganda (J N Opio MPH); Toxicology Unit, Faculty of Pharmacy, University of Port Harcourt, Port Harcourt, Rivers State, Nigeria (Prof O E Orisakwe PhD); Christian Medical College Ludhiana, Ludhiana, India (Prof J D Pandian MD); University of Calgary, Calgary, AB, Canada (Prof S B Patten PhD); AlIMS, New Delhi, India (Prof V K Paul MD); Independent, Waigani, NCD, Papua New Guinea (B I Pavlin $M D)$; 3B's Research Group-Biomaterials, Biodegradables and Biomimetics, University of Minho, Headquarters of the European Institute of Excellence on Tissue Engineering and Regenerative Medicine and ICVS/3B's-PT Government Associate Laboratory, Braga, Portugal (Prof D M Pereira PhD); Postgraduate Medical Institute, Lahore, Punjab, Pakistan (A Pervaiz MHA); Flinders University, Adelaide, SA, Australia (Prof K Pesudovs PhD); Centre for Applied Biostatistics, Sahlgrenska Academy, University of Gothenburg, Gothenburg, Sweden (Prof M Petzold PhD); University of British Columbia, Vancouver, BC, Canada (F Pourmalek PhD); College of Pharmacy, University of Illinois, Chicago, IL, USA (Prof D Qato PhD); Contech Intl., Lahore, Punjab, Pakistan (A Rafay MS); Hamad Medical Corporation, Doha, Qatar (S U Rahman FRCPCH); University of Missouri, Columbia, MO, USA (M Raju PhD); Department of Public Health, University of the Punjab, Lahore, Punjab, Pakistan (S M Rana PhD); Center for Disease Analysis, Louisville, CO, USA (H Razavi PhD); Private consultant, Cairns, QLD, Australia (R Q Reilly $\mathrm{MPH}$ ); IRCCS Mario Negri Institute for Pharmacological Research, Centro Anna Maria Astori, Bergamo, Italy (Prof G Remuzzi MD); Erasmus MC, University Medical Center Rotterdam, Rotterdam, Netherlands (Prof J H Richardus PhD); BARC Hospital, Mumbai, Maharashtra, India (Prof N Roy MD); Rwanda Bio-Medical Center, Kigali, Rwanda (N Sabin MD); National HIV/AIDS \& STI Surveillance and Strategic Information Unit, National Epidemiology Center, Department of Health, Manila, National Capital Region, Philippines (G M J Samonte MD); Marshall University, Huntington, WV, USA M Sawhney PhD); Federal University of Santa Catarina, Florianópolis, Brazil (I J C Schneider PhD); University of Alabama at Birmingham, Birmingham, AL, USA (Prof D C Schwebel PhD, J A Singh MD); Stellenbosch University, Cape Town, Western Cape, South Africa (Prof S Seedat PhD); Health Canada, Ottawa, ON, Canada (H H Shin PhD, S Weichenthal PhD); Heriot-Watt University, Edinburgh, UK (I Shiue PhD); Center for Clinical Global Health Education (R Shivakoti PhD), Johns Hopkins Bloomberg School of Public Health, Johns Hopkins University, Baltimore, MD, USA (B X Tran PhD); Reykjavik University, Reykjavik, Iceland (Prof I D Sigfusdottir PhD); Instituto Nacional de Epidemiología Dr Juan H Jara, Mar del Plata, Buenos Aires, Argentina (A P Silva MgSc); Surveillance and Health Services Research Program American Cancer Society, Atlanta, GA, USA (E P Simard PhD); Dartmouth College, Lebanon, NH, USA (S Soneji PhD); Federal Research Institute for Health Organization and 
Informatics of Ministry of Health of the Russian Federation, Moscow, Russia (S S Soshnikov PhD); Faculty of Medicine and Health Sciences, University Tunku Abdul Rahman, Kajang, Selangor, Malaysia (C T Sreeramareddy MD); Centre Hospitalier Nord Deux-Sevres, Bressuire, France (V K Stathopoulou MD); KEELPNO (Centre for Disease Control, Greece, dispatched to "Alexandra" General Hospital of Athens), Athens, Greece (K Stroumpoulis PhD); National Institute for Research in Tuberculosis, Chennai, Tamil Nadu, India (S Swaminathan MD); Department of Criminology, Law and Society (and Sociology), University of California-Irvine, Chicago, IL, USA (B L Sykes PhD); University of Illinois, Champaign, IL, USA (K M Tabb PhD); Ministry of Health-MINSANTE, Yaounde, Centre, Cameroon (R T Talongwa MD); Memorial University, St John's, NL, Canada (E Y Tenkorang $\mathrm{PhD}$ ); Department of Anesthesiology, University of Virginia, Charlottesville, VA, USA, and Department of Anesthesiology, King Fahad Medical City, Riyadh, Saudi Arabia (A S Terkawi MD); Adaptive Knowledge Management, Victoria, BC, Canada (A J Thomson PhD); The Earth Institute, Columbia University, New York, NY, USA (A L Thorne-Lyman ScD); Cincinnati Children's Hospital Medical Center, Cincinnati, $\mathrm{OH}$, USA (Prof J A Towbin MD); University of Southern Santa Catarina, Palhoça, Santa Catarina, Brazil (Prof J Traebert PhD); Department of Population Sciences and Development, Faculty of Economics and Management, University of Kinshasa, Kinshasa, Democratic Republic of the Congo (Z Tsala Dimbuene PhD); Department of Medicine, University of Crete, Heraklion, Greece (Prof M Tsilimbaris PhD); Department of Veterans Affairs, Washington, DC, USA (U S Uchendu MD); Department of Internal Medicine, Federal Teaching Hospital Abakaliki, Abakailiki, Ebonyi State, Nigeria (K N Ukwaja MD); UKK Institute for Health Promotion Research, Tampere, Finland (Prof T J Vasankari PhD); Neuroscience Centre, Raffles Hospital, Singapore, Singapore (N Venketasubramanian MD); University of Bologna, Bologna, Italy (Prof F S Violante MD); Higher School of Economics, Moscow, Russia (Prof V V Vlassov MD); Uniformed Services University of Health Sciences, Bethesda, MD, USA (S Waller MD); VA Medical Center and Georgetown University Neurology Department, Washington, DC, USA (M T Wallin MD); Shandong University Affiliated Jinan Central Hospital, Jinan, China (X R Wang $\mathrm{PhD}$ ); National Office for Maternal and Child Health Surveillance, Chengdu, China (Prof Y Wang BS, Prof J Zhu MD); University of Marburg, Marburg, Germany (R Westerman PhD); University of Miami, Miami, FL, USA (J D Wilkinson MD); Institute of Public Health, University of Gondar, Gondar, Ethiopia (S M Woldeyohannes MPH); Ateneo School of Medicine and Public Health, Pasig City, Metro Manila, Philippines (J Q Wong MD); Nanjing University School of Medicine, Jinling Hospital, Nanjing, China (Prof G Xu PhD); University of North Carolina at Chapel Hill, Chapel Hill, NC, USA (Y C Yang PhD); Division of Cardiovascular Medicine, Jichi Medical University School of Medicine, Shimotsuke, Tochigi, Japan (Y Yano MD); The University of Hong Kong, Hong Kong, Hong Kong (Prof P Yip $\mathrm{PhD}$ ); National Center of Neurology and Psychiatry, Kodira, Tokyo, Japan (N Yonemoto MPH); Jackson State University, Jackson, MS, USA (Prof M Younis PhD); Department of Epidemiology and Biostatistics, School of Public Health and Global Health Institute, Wuhan University, Wuhan, China (Prof C Yu PhD); TCM 
MEDICAL TK SDN BHD, Nusajaya, Johor Bahru, Malaysia (K Yun Jin PhD);

Mansoura Faculty of Medicine, Mansoura, Egypt (Prof M E S Zaki MD);

Chongqing Medical University, Chongqing, China (Prof $Y$ Zhao MD); Zhongshan

Ophthalmic Center, Sun Yat-sen University, Guangzhou, China (Y Zheng PhD);

Cancer Institute/Hospital, Chinese Academy of Medical Sciences, Beijing, China

(Prof X N Zou MD)

\section{Acknowledgments}

We thank the countless individuals who have contributed to the Global Burden of Disease Study 2013 in various capacities. We would like to acknowledge the extensive support from all staff members at the Institute for Health Metrics and Evaluation and specifically thank: Michael F MacIntyre, Peter Speyer, and Summer Lockett Ohno for their management of the Global Burden of Disease Study 2013; Kelsey Pierce for her valuable assistance; Peter Speyer, James Bullard, Serkan Yalcin, Edgar Sioson, Evan Laurie, Charles Atkinson, and Andrew Ernst for their tireless support of the computational infrastructure necessary to produce the results; Linda A Ettinger for her expert administrative support; Peter Speyer, Abigail McLain, James Hancock, Marissa Iannarone, and Eden Stork for their persistent and invaluable work to gain access to and catalogue as much data as possible to inform the estimates; Stan Biryukov for his assistance with the tuberculosis database; Henry Apfel and Madeline Moyer for data extraction and processing; Christopher Margono, Michelle Subart, Lavanya Singh and Margaret Lind for assistance with galleys; Matthew M Coates, Carly E Levitz, Chelsea A Lidell, Meghan D Mooney, and Austin E Schumaker for production of all-cause mortality estimates; Amelia Bertozzi-Villa for review of Python coding of the Spectrum model. We would also like to acknowledge Mary Mahy, Keith Sabin and John Stover for sharing the UNAIDS EPP files with us and especially John Stover for responding to multiple queries regarding Spectrum. Additionally, Ibrahim Abubakar would like to acknowledge the UK National Institute for Health Research and MRC for funding. Laith J Abu-Raddad would like to acknowledge the support of Qatar National Research Fund (NPRP 04-924-3-251) who provided the main funding for generating the data provided to the GBD-IHME effort. Gabriel Alcalá-Cerra would like to acknowledge institutional support from Health Sciences and Neurosciences (CISNEURO) Research Group. Louisa Degenhardt is supported by an Australian National Health and Medical Research Council Principal Research Fellowship (\#1041742). Dr. Des Jarlais would like to acknowledge funding from NIH Grant DA R01 003574. KD is supported by a Wellcome Trust Fellowship in Public Health and Tropical Medicine [grant number 099876]. Prof. Giuseppe Remuzzi would like to acknowledge the International Society of Nephrology (ISN) for their support of his involvement in the GBD 2013. JAS is supported by grants from the Agency for Health Quality and Research Center for Education and Research on Therapeutics (AHRQ CERTs) U19 HS021110, National Institute of Arthritis, Musculoskeletal and Skin Diseases (NIAMS) P50 AR060772 and U34 AR062891, National Institute of Aging (NIA) U01 AG018947, National Cancer Institute (NCI) U10 CA149950, and research contract CE-1304-6631 from the Patient Centered Outcomes Research Institute (PCORI). JAS is also supported by the resources and the use of facilities at the VA Medical Center at Birmingham, Alabama, USA. Dr. Soneji was supported by the National Center for Advancing Translational Sciences grant number KL2TR001088. Karen M Tabb would like to acknowledge her current support: Lemann Institute Faculty Research Grant, University of Illinois. Andrew J Vallely is supported by a Training Fellowship from the National Health and Medical Research Council (NHMRC), Australia. Gelin Xu is currently funded by the Nature Science Foundation of China (NSFC, 81070922). No individuals acknowledged received additional compensation for their efforts.

\section{References}

1. Institute for Health Metrics and Evaluation. Financing Global Health 2013: Transition in an Age of Austerity. IHME; Seattle, WA: 2013.

2. Floyd K, Fitzpatrick C, Pantoja A, Raviglione M. Domestic and donor financing for tuberculosis care and control in low-income and middle-income countries: an analysis of trends, 2002-11, and requirements to meet 2015 targets. Lancet Glob Health. 2013; 1:e105-15. [PubMed: 25104145]

3. Creese A, Floyd K, Alban A, Guinness L. Cost-effectiveness of HIV/AIDS interventions in Africa: a systematic review of the evidence. Lancet. 2002; 359:1635-43. [PubMed: 12020523]

4. Vassall A, Remme M. Financing tuberculosis control: promising trends and remaining challenges. Lancet Glob Health. 2013; 1:e62-63. [PubMed: 25104150]

5. Hecht R, Stover J, Bollinger L, Muhib F, Case K, de Ferranti D. Financing of HIV/AIDS programme scale-up in low-income and middle-income countries, 2009-31. Lancet. 2010; 376:1254-60. [PubMed: 20934597] 
6. Snow RW, Guerra CA, Mutheu JJ, Hay SI. International funding for malaria control in relation to populations at risk of stable Plasmodium falciparum transmission. PLoS Med. 2008; 5:e142. [PubMed: 18651785]

7. Snow RW, Okiro EA, Gething PW, Atun R, Hay SI. Equity and adequacy of international donor assistance for global malaria control: an analysis of populations at risk and external funding commitments. Lancet. 2010; 376:1409-16. [PubMed: 20889199]

8. Korenromp EL, Hosseini M, Newman RD, Cibulskis RE. Progress towards malaria control targets in relation to national malaria programme funding. Malar J. 2013; 12:18. [PubMed: 23317000]

9. Waddington, C.; Martin, J.; Walford, V. Trends in International Funding for Malaria Control. HLSP Institute; London, UK: 2005. http://rbm.who.int/docs/hlsp_report.pdf [accessed February 26, 2014]

10. Waage J, Banerji R, Campbell O, et al. The Millennium Development Goals: a cross-sectoral analysis and principles for goal setting after 2015 Lancet and London International Development Centre Commission. Lancet. 2010; 376:991-1023. [PubMed: 20833426]

11. Dye C, Glaziou P, Floyd K, Raviglione M. Prospects for tuberculosis elimination. Annu Rev Public Health. 2013; 34:271-86. [PubMed: 23244049]

12. United Nations. The Millennium Development Goals Report 2013. United Nations; New York, NY: 2013. http://www.un.org/millenniumgoals/pdf/report-2013/mdg-report-2013-english.pdf [accessed February 26, 2014]

13. Clemens MA, Kenny CJ, Moss TJ. The Trouble with the MDGs: Confronting Expectations of Aid and Development Success. World Dev. 2007; 35:735-51.

14. Atun R, Raviglione M, Marais B, Zumla A. Tuberculosis control is crucial to achieve the MDGs. Lancet. 2010; 376:940-41. [PubMed: 20851247]

15. Lomazzi M, Borisch B, Laaser U. The Millennium Development Goals: experiences, achievements and what's next. Glob Health Action. 2014; 7:23695. [PubMed: 24560268]

16. Fehling M, Nelson BD, Venkatapuram S. Limitations of the Millennium Development Goals: a literature review. Glob Public Health. 2013; 8:1109-22. [PubMed: 24266508]

17. Stuckler D, Basu S, McKee M. Drivers of inequality in Millennium Development Goal progress: a statistical analysis. PLoS Med. 2010; 7:e1000241. [PubMed: 20209000]

18. Vitoria M, Granich R, Gilks CF, et al. The global fight against HIV/AIDS, tuberculosis, and malaria: current status and future perspectives. Am J Clin Pathol. 2009; 131:844-48. [PubMed: 19461091]

19. Alleyne G, Binagwaho A, Haines A, et al. Lancet NCD Action Group. Embedding noncommunicable diseases in the post-2015 development agenda. Lancet. 2013; 381:566-74. [PubMed: 23410606]

20. MEDICC. NCDs: can healthy synergies replace fatal interactions? MEDICC Rev. 2013; 15:3.

21. WHO. WEF. From Burden to 'Best Buys': Reducing the Economic Impact of Non-Communicable Diseases in Low- and Middle-Income Countries. World Economic Forum; 2011. http:// www3.weforum.org/docs/WEF_WHO_HE_ReducingNonCommunicableDiseases_2011.pdf [accessed February 26, 2014]

22. WEF. Harvard School of Public Health. Glob. Econ. Burd. Non-Commun. Dis. World Econ. Forum; The Global Economic Burden of Non-communicable Diseases. http://www.weforum.org/ reports/global-economic-burden-non-communicable-diseases [accessed February 27, 2014]

23. [accessed February 27, 2014] 2011 High Level Meeting on the Prevention and Control of Noncommunicable Diseases. https://www.un.org/en/ga/ncdmeeting2011/

24. Alliance NCD. Tackling Non-communicable Diseases to Enhance Sustainable Development. NCD Alliance; 2014. http://ncdalliance.org/sites/default/files/NCD\%20Alliance\%20-\%20NCDs\%20and \%20Sustainable\%20Development\%20Brief_0.pdf [accessed February 26, 2014]

25. Beaglehole R, Bonita R, Horton R, et al. Lancet NCD Action Group; NCD Alliance. Priority actions for the non-communicable disease crisis. Lancet. 2011; 377:1438-47. [PubMed: 21474174]

26. Abegunde DO, Mathers CD, Adam T, Ortegon M, Strong K. The burden and costs of chronic diseases in low-income and middle-income countries. Lancet. 2007; 370:1929-38. [PubMed: 18063029] 
27. Shiffman J. Has donor prioritization of HIV/AIDS displaced aid for other health issues? Health Policy Plan. 2008; 23:95-100. [PubMed: 18156161]

28. Vandemoortele J, Delamonica E. Taking the MDGs Beyond 2015: Hasten Slowly. IDS Bull. 2010; 41:60-69.

29. UNAIDS. 2011-2015 Strategy: Getting to Zero. Joint United Nations Programme on HIV/AIDS; 2010. http://www.unaids.org/en/media/unaids/contentassets/documents/unaidspublication/2010/ JC2034_UNAIDS_Strategy_en.pdf

30. Treatment Action Group. [accessed March 5, 2014] The Zero Declaration. 2012. http:// www.treatmentactiongroup.org/tb/advocacy/zero-declaration

31. Department for International Development. UKAID. Towards zero infections: The UK's position paper on HIV in the developing world. 2011. https://www.gov.uk/government/uploads/system/ uploads/attachment_data/file/67523/twds-zero-infs-pos-paper-hiv-dev-wrld.pdf

32. South Africa Info Reporter. South Africa's HIV/Aids battle plan. 2012. SouthAfrica.infohttp:// www.southafrica.info/about/health/aids-prevention.htm\#.UxZ1nfldXg9

33. WHO. Roadmap for Childhood Tuberculosis. 2013. http://apps.who.int/iris/bitstream/ 10665/89506/1/9789241506137_eng.pdf

34. Stop, TB. Towards Zero TB Deaths In Children. WHO; 2012. Partnership. No More Crying, No More Dying. http://www.stoptb.org/assets/documents/news/ChildhoodTB_report_singles.pdf

35. Ki-moon, B. [accessed March 5, 2014] Secretary-General's message on World Malaria Day. 2011. un.orghttp://www.un.org/sg/statements/?nid=5219

36. Wagstaff, A. The Millennium Development Goals for Health: Rising to the Challenges. World Bank Publications; 2004.

37. UNAIDS. AIDSinfo Database. Joint United Nations Programme on HIV/AIDS; 2013. http:// www.aidsinfoonline.org/devinfo/libraries/aspx/Home.aspx

38. Stover J, Brown T, Marston M. Updates to the Spectrum/Estimation and Projection Package (EPP) model to estimate HIV trends for adults and children. Sex Transm Infect. 2012; 88(suppl 2):i1116. [PubMed: 23172341]

39. UNAIDS. Methodology - Understanding the HIV estimates. Geneva, Switzerland: 2013. http:// www.unaids.org/en/media/unaids/contentassets/documents/epidemiology/2013/ gr2013/20131118_Methodology.pdf

40. Pretorius C, Menzies NA, Chindelevitch L, et al. The potential effects of changing HIV treatment policy on tuberculosis outcomes in South Africa: results from three tuberculosis-HIV transmission models. AIDS. 2014; 28(suppl 1):S25-34. [PubMed: 24468944]

41. Murray CJ, Ezzati M, Flaxman AD, et al. GBD 2010: a multi-investigator collaboration for global comparative descriptive epidemiology. Lancet. 2012; 380:2055-58. [PubMed: 23245598]

42. Murray CJ, Ezzati M, Flaxman AD, et al. GBD 2010: design, definitions, and metrics. Lancet. 2012; 380:2063-66. [PubMed: 23245602]

43. Wang H, Dwyer-Lindgren L, Lofgren KT, et al. Age-specific and sex-specific mortality in 187 countries, 1970-2010: a systematic analysis for the Global Burden of Disease Study 2010. Lancet. 2012; 380:2071-94. [PubMed: 23245603]

44. Salomon JA, Vos T, Hogan DR, et al. Common values in assessing health outcomes from disease and injury: disability weights measurement study for the Global Burden of Disease Study 2010. Lancet. 2012; 380:2129-43. [PubMed: 23245605]

45. Salomon JA, Wang H, Freeman MK, et al. Healthy life expectancy for 187 countries, 1990-2010: a systematic analysis for the Global Burden Disease Study 2010. Lancet. 2012; 380:2144-62. [PubMed: 23245606]

46. Vos T, Flaxman AD, Naghavi M, et al. Years lived with disability (YLDs) for 1160 sequelae of 289 diseases and injuries 1990-2010: a systematic analysis for the Global Burden of Disease Study 2010. Lancet. 2012; 380:2163-96. [PubMed: 23245607]

47. Murray CJL, Vos T, Lozano R, et al. Disability-adjusted life years (DALYs) for 291 diseases and injuries in 21 regions, 1990-2010: a systematic analysis for the Global Burden of Disease Study 2010. Lancet. 2012; 380:2197-223. [PubMed: 23245608]

48. Lim SS, Vos T, Flaxman AD, et al. A comparative risk assessment of burden of disease and injury attributable to 67 risk factors and risk factor clusters in 21 regions, 1990-2010: a systematic 
analysis for the Global Burden of Disease Study 2010. Lancet. 2012; 380:2224-60. [PubMed: 23245609]

49. The GBD 2013 Collaboration. Global, Regional, and National Burden of Disease for 322 Disease and Injuries and 68 Risk Factors, 1990-2013: A Systematic Analysis. In preparation.

50. The GBD 2013 Collaboration. Global, Regional, and National Levels of Age-Specific Mortality and 266 Causes of Death, 1990-2013: A Systematic Analysis. In preparation.

51. The GBD 2013 Collaboration. Global, Regional, and National Prevalence and Disability for 318 Disease and Injuries and Their 2,435 Sequelae, 1990-2013: A Systematic Analysis. In preparation.

52. Ghys PD, Brown T, Grassly NC, et al. The UNAIDS Estimation and Projection Package: a software package to estimate and project national HIV epidemics. Sex Transm Infect. 2004; 80(suppl 1):i5-9. [PubMed: 15249692]

53. Brown T, Grassly NC, Garnett G, Stanecki K. Improving projections at the country level: the UNAIDS Estimation and Projection Package 2005. Sex Transm Infect. 2006; 82(suppl 3):iii34-40. [PubMed: 16735291]

54. Todd J, Glynn JR, Marston M, et al. Time from HIV seroconversion to death: a collaborative analysis of eight studies in six low and middle-income countries before highly active antiretroviral therapy. AIDS. 2007; 21(suppl 6):S55-63. [PubMed: 18032940]

55. Ghys PD, Zaba B, Prins M. Survival and mortality of people infected with HIV in low and middle income countries: results from the extended ALPHA network. AIDS. 2007; 21(suppl 6):S1-4. [PubMed: 18032932]

56. Hallett TB, Zaba B, Todd J, et al. ALPHA Network. Estimating incidence from prevalence in generalised HIV epidemics: methods and validation. PLoS Med. 2008; 5:e80. [PubMed: 18590346]

57. McGowan CC, Cahn P, Gotuzzo E, et al. Cohort Profile: Caribbean, Central and South America Network for HIV research (CCASAnet) collaboration within the International Epidemiologic Databases to Evaluate AIDS (IeDEA) programme. Int J Epidemiol. 2007; 36:969-76. [PubMed: 17846055]

58. Nash D, Katyal M, Brinkhof MWG, et al. ART-LINC Collaboration of IeDEA. Long-term immunologic response to antiretroviral therapy in low-income countries: a collaborative analysis of prospective studies. AIDS. 2008; 22:2291-302. [PubMed: 18981768]

59. Verguet S, Lim SS, Murray CJL, Gakidou E, Salomon JA. Incorporating loss to follow-up in estimates of survival among HIV-infected individuals in sub-Saharan Africa enrolled in antiretroviral therapy programs. J Infect Dis. 2013; 207:72-79. [PubMed: 23100567]

60. UNAIDS. Global Report: UNAIDS report on the global AIDS epidemic 2013. Joint United Nations Programme on HIV/AIDS; 2013. http://www.unaids.org/en/media/unaids/contentassets/ documents/epidemiology/2013/gr2013/UNAIDS_Global_Report_2013_en.pdf [accessed 26 Feb 2014]

61. WHO. Case Study on Estimating HIV Infection in a Concentrated Epidemic: Lessons from Indonesia. WHO; 2004. http://www.who.int/hiv/pub/epidemiology/indonesia/en/ [accessed February 27, 2014]

62. Papworth E, Ceesay N, An L, et al. Epidemiology of HIV among female sex workers, their clients, men who have sex with men and people who inject drugs in West and Central Africa. J Int AIDS Soc. 2013; 16(suppl 3):18751. [PubMed: 24321113]

63. Bautista-Arredondo S, Colchero MA, Romero M, Conde-Glez CJ, Sosa-Rubí SG. Is the HIV epidemic stable among MSM in Mexico? HIV prevalence and risk behavior results from a nationally representative survey among men who have sex with men. PLoS ONE. 2013; 8:e72616. [PubMed: 24039786]

64. Ruan Y, Liang S, Zhu J, et al. Gender and ethnic disparities ofHIV and syphilis seroconversions in a 4-year cohort of injection drug users. Southeast Asian J Trop Med Public Health. 2013; 44:84253. [PubMed: 24437319]

65. Decker MR, Wirtz AL, Moguilnyi V, et al. Female Sex Workers in Three Cities in Russia: HIV Prevalence, Risk Factors and Experience with Targeted HIV Prevention. AIDS Behav. 2014; 18:562-72. [PubMed: 23929034] 
66. Miller WM, Buckingham L, Sánchez-Domínguez MS, Morales-Miranda S, Paz-Bailey G. Systematic review of HIV prevalence studies among key populations in Latin America and the Caribbean. Salud Publica Mex. 2013; 55(suppl 1):S65-78. [PubMed: 23918059]

67. Mason K, Ketende S, Peitzmeier S, et al. A cross-sectional analysis of population demographics, HIV knowledge and risk behaviors, and prevalence and associations of HIV among men who have sex with men in the Gambia. AIDS Res Hum Retroviruses. 2013; 29:1547-52. [PubMed: 23875674]

68. Li D, Wang L, Lin W, et al. HIV and syphilis infections among street-based female sex workers in China, 2010-2012. Chin Med J (Engl). 2014; 127:707-11. [PubMed: 24534227]

69. Sajadi L, Mirzazadeh A, Navadeh S, et al. HIV prevalence and related risk behaviours among female sex workers in Iran: results of the national biobehavioural survey, 2010. Sex Transm Infect. 2013; 89(suppl 3):iii37-40. [PubMed: 24191292]

70. Mathers BM, Degenhardt L, Ali H, et al. 2009 Reference Group to the UN on HIV and Injecting Drug Use. HIV prevention, treatment, and care services for people who inject drugs: a systematic review of global, regional, and national coverage. Lancet. 2010; 375:1014-28. [PubMed: 20189638]

71. Mathers BM, Degenhardt L, Phillips B, et al. 2007 Reference Group to the UN on HIV and Injecting Drug Use. Global epidemiology of injecting drug use and HIV among people who inject drugs: a systematic review. Lancet. 2008; 372:1733-45. [PubMed: 18817968]

72. Prüss-Ustün A, Wolf J, Driscoll T, Degenhardt L, Neira M, Calleja JMG. HIV due to female sex work: regional and global estimates. PLoS ONE. 2013; 8:e63476. [PubMed: 23717432]

73. UNAIDS. WHO. Guidelines for measuring national HIV prevalence in population-based surveys. Joint United Nations Programme on HIV/AIDS; Geneva: 2005. http://data.unaids.org/pub/manual/ 2005/20050101_gs_guidemeasuringpopulation_en.pdf

74. Lozano R, Naghavi M, Foreman K, et al. Global and regional mortality from 235 causes of death for 20 age groups in 1990 and 2010: a systematic analysis for the Global Burden of Disease Study 2010. Lancet. 2012; 380:2095-128. [PubMed: 23245604]

75. Birnbaum JK, Murray CJ, Lozano R. Exposing misclassified HIV/AIDS deaths in South Africa. Bull World Health Organ. 2011; 89:278-85. [PubMed: 21479092]

76. Foreman KJ, Lozano R, Lopez AD, Murray CJ. Modeling causes of death: an integrated approach using CODEm. Popul Health Metr. 2012; 10:1. [PubMed: 22226226]

77. Ng M, Freeman MK, Fleming TD, et al. Smoking prevalence and cigarette consumption in 187 countries, 1980-2012. JAMA. 2014; 311:183-92. [PubMed: 24399557]

78. USAID. Demographic Health Surveys Program. http://www.dhsprogram.com/

79. International Institute for Population Sciences. National Family Health Survey, India. Mumbai, India: http://www.rchiips.org/nfhs/

80. Simbayi, L.; Shisana, O.; Rehle, T.; et al. Human Sciences Resource Council. South African National HIV Prevalence, Incidence and Behaviour Survey, 2012. 2014. http://www.hsrc.ac.za/en/ research-outputs/view/6871

81. Dye C, Lönnroth K, Jaramillo E, Williams BG, Raviglione M. Trends in tuberculosis incidence and their determinants in 134 countries. Bull World Health Organ. 2009; 87:683-91. [PubMed: 19784448]

82. WHO. Global tuberculosis report 2013. 2013. http://apps.who.int/iris/bitstream/ 10665/91355/1/9789241564656_eng.pdf

83. WHO. Tuberculosis prevalence surveys: a handbook. WHO; 2011. http://www.who.int/tb/ advisory_bodies/impact_measurement_taskforce/resources_documents/thelimebook/en/ [accessed February 26, 2014]

84. WHO. Global Task Force on TB Impact Measurement. National TB prevalence surveys; 2014.

85. Naghavi M, Makela S, Foreman K, O’Brien J, Pourmalek F, Lozano R. Algorithms for enhancing public health utility of national causes-of-death data. Popul Health Metr. 2010; 8:9. [PubMed: 20459720]

86. Sonnenberg P, Glynn JR, Fielding K, Murray J, Godfrey-Faussett P, Shearer S. How soon after infection with HIV does the risk of tuberculosis start to increase? A retrospective cohort study in South African gold miners. J Infect Dis. 2005; 191:150-58. [PubMed: 15609223] 
87. Allen S, Batungwanayo J, Kerlikowske K, et al. Two-year incidence of tuberculosis in cohorts of HIV-infected and uninfected urban Rwandan women. Am Rev Respir Dis. 1992; 146:1439-44. [PubMed: 1456559]

88. Braun MM, Badi N, Ryder RW, et al. A retrospective cohort study of the risk of tuberculosis among women of childbearing age with HIV infection in Zaire. Am Rev Respir Dis. 1991; 143:501-04. [PubMed: 2001057]

89. Keizer ST, Langendam MM, van Deutekom H, Coutinho RA, van Ameijden EJC. How does tuberculosis relate to HIV positive and HIV negative drug users? J Epidemiol Community Health. 2000; 54:64-68. [PubMed: 10692965]

90. Leroy V, Msellati P, Lepage P, et al. Four years of natural history of HIV-1 infection in african women: a prospective cohort study in Kigali (Rwanda), 1988-1993. J Acquir Immune Defic Syndr Hum Retrovirol. 1995; 9:415-21. [PubMed: 7600110]

91. Houben RMGJ, Glynn JR, Mboma S, et al. The impact of HIV and ART on recurrent tuberculosis in a sub-Saharan setting. AIDS. 2012; 26:2233-39. [PubMed: 22951633]

92. Ferreira MM, Ferrazoli L, Palaci M, et al. Tuberculosis and HIV infection among female inmates in São Paulo, Brazil: a prospective cohort study. J Acquir Immune Defic Syndr Hum Retrovirol. 1996; 13:177-83. [PubMed: 8862283]

93. Badri M, Wilson D, Wood R. Effect of highly active antiretroviral therapy on incidence of tuberculosis in South Africa: a cohort study. Lancet. 2002; 359:2059-64. [PubMed: 12086758]

94. Murray CJL, Rosenfeld LC, Lim SS, et al. Global malaria mortality between 1980 and 2010: a systematic analysis. Lancet. 2012; 379:413-31. [PubMed: 22305225]

95. Byass P, de Courten M, Graham WJ, et al. Reflections on the global burden of disease 2010 estimates. PLoS Med. 2013; 10:e1001477. [PubMed: 23843748]

96. Lynch M, Korenromp E, Eisele T, et al. New global estimates of malaria deaths. Lancet. 2012; 380:559. [PubMed: 22883496]

97. Bates M, O'Grady J, Mudenda V, Shibemba A, Zumla A. New global estimates of malaria deaths. Lancet. 2012; 380:560-61. [PubMed: 22883498]

98. Chambers RG. UN Envoy's response to estimates of global malaria mortality. Lancet. 2012; 379:707-08. [PubMed: 22357104]

99. Shah NK, Kumar A, Valecha N. New global estimates of malaria deaths. Lancet. 2012; 380:560. [PubMed: 22883499]

100. White NJ, Dondorp AM, Faiz A, Mishra S, Hien TT. New global estimates of malaria deaths. Lancet. 2012; 380:559-60. [PubMed: 22883497]

101. Snow RW, Armstrong JR, Forster D, et al. Childhood deaths in Africa: uses and limitations of verbal autopsies. Lancet. 1992; 340:351-55. [PubMed: 1353814]

102. Mpimbaza A, Filler S, Katureebe A, et al. Validity of verbal autopsy procedures for determining malaria deaths in different epidemiological settings in Uganda. PLoS ONE. 2011; 6:e26892. [PubMed: 22046397]

103. Nykanen M, Tamaona W, Cullinan T, Van Oosterzee V, Ashorn P. Verbal autopsy as a technique to establish causes of infant and child mortality. East Afr Med J. 1995; 72:731-34. [PubMed: 8904066]

104. Todd JE, De Francisco A, O’Dempsey TJ, Greenwood BM. The limitations of verbal autopsy in a malaria-endemic region. Ann Trop Paediatr. 1994; 14:31-36. [PubMed: 7516132]

105. Setel PW, Whiting DR, Hemed Y, et al. Validity of verbal autopsy procedures for determining cause of death in Tanzania. Trop Med Int Health. 2006; 11:681-96. [PubMed: 16640621]

106. Chandramohan D, Maude GH, Rodrigues LC, Hayes RJ. Verbal autopsies for adult deaths: their development and validation in a multicentre study. Trop Med Int Health. 1998; 3:436-46. [PubMed: 9657505]

107. Murray CJL, Lozano R, Flaxman AD, et al. Using verbal autopsy to measure causes of death: the comparative performance of existing methods. BMC Med. 2014; 12:5. [PubMed: 24405531]

108. Gething PW, Patil AP, Smith DL, et al. A new world malaria map: Plasmodium falciparum endemicity in 2010. Malar J. 2011; 10:378. [PubMed: 22185615] 
109. Dieleman JL, Graves CM, Templin T, et al. Global health development assistance remained steady in 2013 but did not align with recipients' disease burden. Health Aff (Millwood). 2014; 33:878-86. [PubMed: 24714869]

110. WHO. [accessed 10 Mar 2014] World Malaria Report. 2013. 2013. http://www.who.int/malaria/ publications/world_malaria_report_2013/report/en/

111. Yang X, Jiao YM, Wang R, et al. High CCR5 density on central memory CD4+ T cells in acute HIV-1 infection is mostly associated with rapid disease progression. PLoS ONE. 2012; 7:e49526. [PubMed: 23185351]

112. International HIV Controllers Study. Pereyra F, Jia X, et al. The major genetic determinants of HIV-1 control affect HLA class I peptide presentation. Science. 2010; 330:1551-57. [PubMed: 21051598]

113. Herbeck JT, Gottlieb GS, Winkler CA, et al. Multistage genomewide association study identifies a locus at 1q41 associated with rate of HIV-1 disease progression to clinical AIDS. J Infect Dis. 2010; 201:618-26. [PubMed: 20064070]

114. Freguja R, Gianesin K, Del Bianco P, et al. Polymorphisms of innate immunity genes influence disease progression in HIV-1-infected children. AIDS. 2012; 26:765-68. [PubMed: 22269973]

115. Freguja R, Gianesin K, Zanchetta M, De Rossi A. Cross-talk between virus and host innate immunity in pediatric HIV-1 infection and disease progression. New Microbiol. 2012; 35:24957. [PubMed: 22842595]

116. Hewitt K, Steketee R, Mwapasa V, Whitworth J, French N. Interactions between HIV and malaria in non-pregnant adults: evidence and implications. AIDS. 2006; 20:1993-2004. [PubMed: 17053345]

117. Flateau C, Le Loup G, Pialoux G. Consequences of HIV infection on malaria and therapeutic implications: a systematic review. Lancet Infect Dis. 2011; 11:541-56. [PubMed: 21700241]

118. Umunyana, J. [accessed 11 Apr 2014] Incidence of Malaria in HIV-infected and uninfected and Rwandan women from 2005 to 2011. 2014. http://wiredspace.wits.ac.za/handle/10539/14502

119. UNAIDS. 2007 AIDS Epidemic Update. 2007. http://data.unaids.org/pub/epislides/ 2007/2007_epiupdate_en.pdf

120. Donnell D, Baeten JM, Kiarie J, et al. Partners in Prevention HSV/HIV Transmission Study Team. Heterosexual HIV-1 transmission after initiation of antiretroviral therapy: a prospective cohort analysis. Lancet. 2010; 375:2092-98. [PubMed: 20537376]

121. Attia S, Egger M, Müller M, Zwahlen M, Low N. Sexual transmission of HIV according to viral load and antiretroviral therapy: systematic review and meta-analysis. AIDS. 2009; 23:1397-404. [PubMed: 19381076]

122. Jackson DJ, Chopra M, Doherty TM, et al. Good Start Study Group. Operational effectiveness and 36 week HIV-free survival in the South African programme to prevent mother-to-child transmission of HIV-1. AIDS. 2007; 21:509-16. [PubMed: 17301570]

123. Dabis F, Bequet L, Ekouevi DK, et al. ANRS 1201/1202 DITRAME PLUS Study Group. Field efficacy of zidovudine, lamivudine and single-dose nevirapine to prevent peripartum HIV transmission. AIDS. 2005; 19:309-18. [PubMed: 15718842]

124. Mermin J, Lule J, Ekwaru JP, et al. Effect of co-trimoxazole prophylaxis on morbidity, mortality, CD4-cell count, and viral load in HIV infection in rural Uganda. Lancet. 2004; 364:1428-34. [PubMed: 15488218]

125. Chintu C, Bhat GJ, Walker AS, et al. CHAP trial team. Co-trimoxazole as prophylaxis against opportunistic infections in HIV-infected Zambian children (CHAP): a double-blind randomised placebo-controlled trial. Lancet. 2004; 364:1865-71. [PubMed: 15555666]

126. Freedberg KA, Losina E, Weinstein MC, et al. The cost effectiveness of combination antiretroviral therapy for HIV disease. N Engl J Med. 2001; 344:824-31. [PubMed: 11248160]

127. Goldie SJ, Yazdanpanah Y, Losina E, et al. Cost-effectiveness of HIV treatment in resource-poor settings - the case of Côte d'Ivoire. N Engl J Med. 2006; 355:1141-53. [PubMed: 16971720]

128. Cleary SM, McIntyre D, Boulle AM. The cost-effectiveness of antiretroviral treatment in Khayelitsha, South Africa-a primary data analysis. Cost Eff Resour Alloc. 2006; 4:20. [PubMed: 17147833] 
129. Degenhardt L, Mathers BM, Wirtz AL, et al. What has been achieved in HIV prevention, treatment and care for people who inject drugs, 2010-2012? A review of the six highest burden countries. Int J Drug Policy. 2014; 25:53-60. [PubMed: 24113623]

130. Narasimhan P, Wood J, Macintyre CR, Mathai D. Risk factors for tuberculosis. Pulm Med. 2013; 2013:828939. DOI:10.1155/2013/828939. [PubMed: 23476764]

131. Bates MN, Khalakdina A, Pai M, Chang L, Lessa F, Smith KR. Risk of tuberculosis from exposure to tobacco smoke: a systematic review and meta-analysis. Arch Intern Med. 2007; 167:335-42. [PubMed: 17325294]

132. Lönnroth K, Jaramillo E, Williams BG, Dye C, Raviglione M. Drivers of tuberculosis epidemics: the role of risk factors and social determinants. Soc Sci Med. 2009; 68:2240-46. [PubMed: 19394122]

133. Espinal MA. The global situation of MDR-TB. Tuberculosis (Edinb). 2003; 83:44-51. [PubMed: 12758188]

134. Zignol M, Hosseini MS, Wright A, et al. Global incidence of multidrug-resistant tuberculosis. J Infect Dis. 2006; 194:479-85. [PubMed: 16845631]

135. Zhao Y, Xu S, Wang L, et al. National survey of drug-resistant tuberculosis in China. N Engl J Med. 2012; 366:2161-70. [PubMed: 22670902]

136. WHO. Global tuberculosis control 2010. http://www.who.int/tb/publications/global_report/ 2010/en/

137. Mehra M, Cossrow N, Kambili C, Underwood R, Makkar R, Potluri R. Assessment of tuberculosis burden in China using a dynamic disease simulation model. Int J Tuberc Lung Dis. 2013; 17:1186-94. [PubMed: 23827732]

138. Malaria Funding \& Resource Utilization: The First Decade of Roll Back Malaria. 2010. Roll Back Malaria.

139. Otten M, Aregawi M, Were W, et al. Initial evidence of reduction of malaria cases and deaths in Rwanda and Ethiopia due to rapid scale-up of malaria prevention and treatment. Malar J. 2009; 8:14. [PubMed: 19144183]

140. Steketee RW, Campbell CC. Impact of national malaria control scale-up programmes in Africa: magnitude and attribution of effects. Malar J. 2010; 9:299. [PubMed: 20979634]

141. O’Meara WP, Mangeni JN, Steketee R, Greenwood B. Changes in the burden of malaria in subSaharan Africa. Lancet Infect Dis. 2010; 10:545-55. [PubMed: 20637696]

142. Noor AM, Kinyoki DK, Mundia CW, et al. The changing risk of Plasmodium falciparum malaria infection in Africa: 2000-10: a spatial and temporal analysis of transmission intensity. Lancet. 2014 DOI:10.1016/S0140-6736(13)62566-0.

143. WHO. Q \& A on malaria mortality estimates. 2012. http://www.searo.who.int/entity/malaria/ WHOGMP_burden_estimates_qa.pdf

144. Shah NK. Defining \& counting malaria deaths. Indian J Med Res. 2012; 135:270-72. [PubMed: 22561610]

145. Shah NK, Dhariwal AC, Sonal GS, Gunasekar A, Dye C, Cibulskis R. Malaria-attributed death rates in India. Lancet. 2011; 377:991. author reply 994-95. [PubMed: 21420545]

146. Crump JA, Morrissey AB, Nicholson WL, et al. Etiology of severe non-malaria febrile illness in Northern Tanzania: a prospective cohort study. PLoS Negl Trop Dis. 2013; 7:e2324. [PubMed: 23875053]

147. Reyburn H, Mbatia R, Drakeley C, et al. Overdiagnosis of malaria in patients with severe febrile illness in Tanzania: a prospective study. BMJ. 2004; 329:1212. [PubMed: 15542534]

148. Nadjm B, Mtove G, Amos B, et al. Severe febrile illness in adult hospital admissions in Tanzania: a prospective study in an area of high malaria transmission. Trans R Soc Trop Med Hyg. 2012; 106:688-95. [PubMed: 23022040]

149. Makani J, Matuja W, Liyombo E, Snow RW, Marsh K, Warrell DA. Admission diagnosis of cerebral malaria in adults in an endemic area of Tanzania: implications and clinical description. QJM. 2003; 96:355-62. [PubMed: 12702784]

150. Snow RW, Korenromp EL, Gouws E. Pediatric mortality in Africa: plasmodium falciparum malaria as a cause or risk? Am J Trop Med Hyg. 2004; 71(suppl):16-24. [PubMed: 15331815] 
151. Scott JAG, Berkley JA, Mwangi I, et al. Relation between falciparum malaria and bacteraemia in Kenyan children: a population-based, case-control study and a longitudinal study. Lancet. 2011; 378:1316-23. [PubMed: 21903251]

152. Shanks GD, Hay SI, Bradley DJ. Malaria's indirect contribution to all-cause mortality in the Andaman Islands during the colonial era. Lancet Infect Dis. 2008; 8:564-70. [PubMed: 18599354]

153. Gray RH. The decline of mortality in Ceylon and the demographic effects of malaria control. Popul Stud (Camb). 1974; 28:205-29. [PubMed: 11630544]

154. Giglioli G. Changes in the pattern of mortality following the eradication of hyperendemic malaria from a highly susceptible community. Bull World Health Organ. 1972; 46:181-202. [PubMed: 4624339]

155. D'Alessandro U, Olaleye BO, McGuire W, et al. Mortality and morbidity from malaria in Gambian children after introduction of an impregnated bednet programme. Lancet. 1995; 345:479-83. [PubMed: 7861874]

156. Alonso PL, Lindsay SW, Armstrong JR, et al. The effect of insecticide-treated bed nets on mortality of Gambian children. Lancet. 1991; 337:1499-502. [PubMed: 1675368]

157. Darby SC, Ewart DW, Giangrande PL, Spooner RJ, Rizza CR, UK Haemophilia Centre Directors' Organisation. Importance of age at infection with HIV-1 for survival and development of AIDS in UK haemophilia population. Lancet. 1996; 347:1573-79. [PubMed: 8667864]

158. Ceesay SJ, Casals-Pascual C, Erskine J, et al. Changes in malaria indices between 1999 and 2007 in The Gambia: a retrospective analysis. Lancet. 2008; 372:1545-54. [PubMed: 18984187]

159. Ceesay SJ, Casals-Pascual C, Nwakanma DC, et al. Continued decline of malaria in The Gambia with implications for elimination. PLoS ONE. 2010; 5:e12242. [PubMed: 20805878]

160. Futures Institute. [accessed Feb 26, 2014] AIM: a computer program for making HIV/AIDS projections and examining the demographic and social impacts of AIDS. https:// spectrummodel.zendesk.com/attachments/token/6eixzeawlonxjg7/?name=AIM $+2014+$ English.pdf

161. Liu L, Johnson HL, Cousens S, et al. Global, regional, and national causes of child mortality: an updated systematic analysis for 2010 with time trends since 2000. Lancet. 2012; 379:2151-61. [PubMed: 22579125]

162. WHO. [accessed Jun 12, 2014] World Malaria Report 2008. http://www.who.int/malaria/ publications/atoz/9789241563697/en/

163. Hay SI, Okiro EA, Gething PW, et al. Estimating the Global Clinical Burden of Plasmodium falciparum Malaria in 2007. PLoS Med. 2010; 7:e1000290. [PubMed: 20563310]

164. Snow, RW.; Newton, CRJC. The public health burden of Plasmodium falciparum malaria in Africa: deriving the numbers. The Disease Control Priorities Project (DCPP); Washington DC: 2003. p. 75Working Paper Number 11 


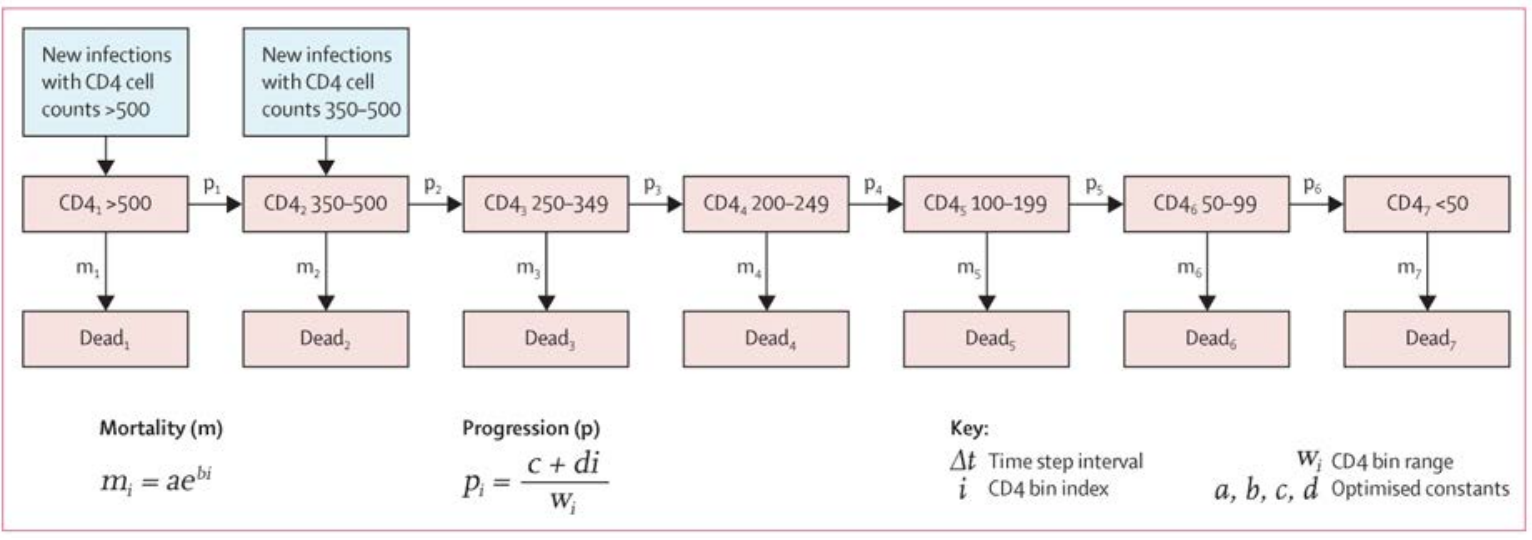

Figure 1. UNAIDS compartmental model for estimating mortality in HIV-positive individuals in the absence of ART

ART=antiretroviral therapy. 


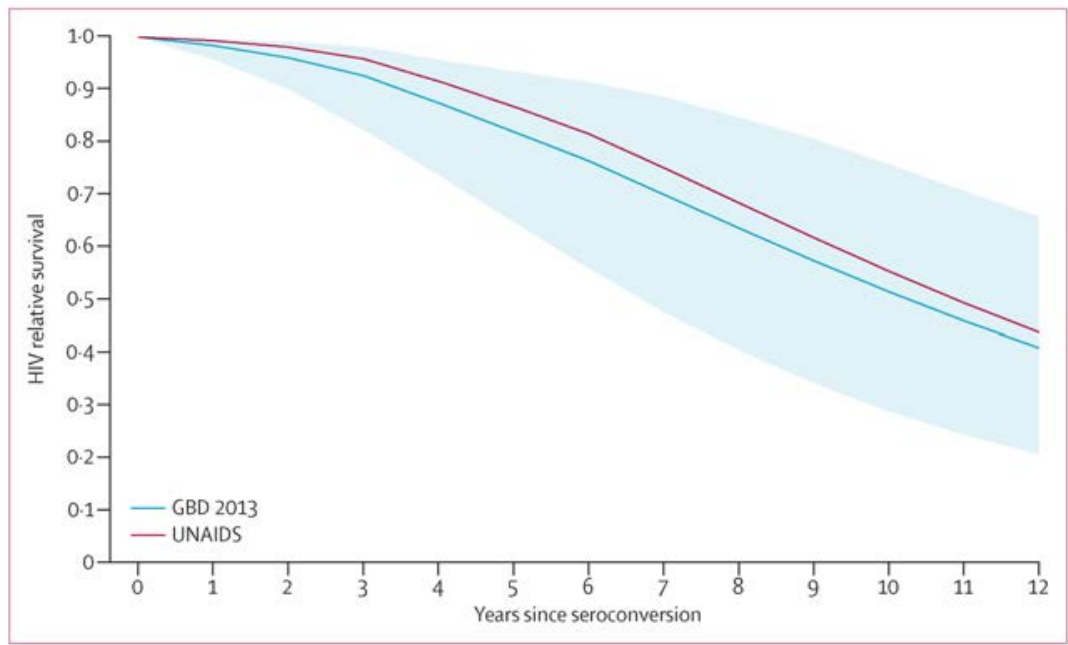

Figure 2. HIV relative survival after seroconversion for male and female individuals aged 25-34 years based on the analysis of 13 ART-naive cohort studies

Solid lines show means; shaded area shows 95\% uncertainty intervals (uncertainty intervals not available for UNAIDS data). ART=antiretroviral therapy. 


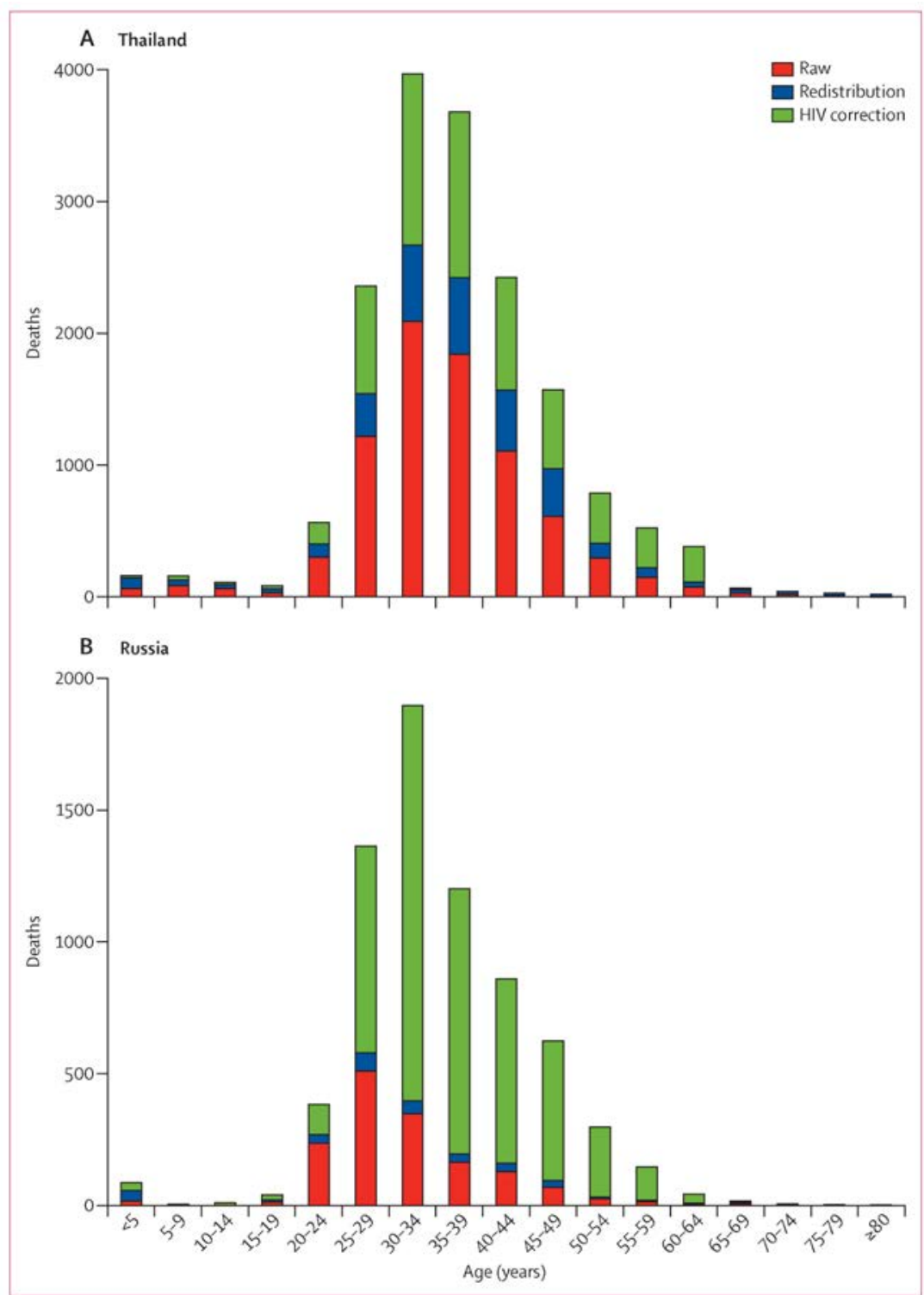

Figure 3. HIV deaths in Thailand (A) and Russia (B) by age in 2005 for both sexes combined Data are for vital registration deaths assigned to HIV (red), deaths coded to garbage codes redistributed to HIV (blue), and misclassified deaths reassigned to HIV (green). The height of the bar is the final number of deaths in each age group. 


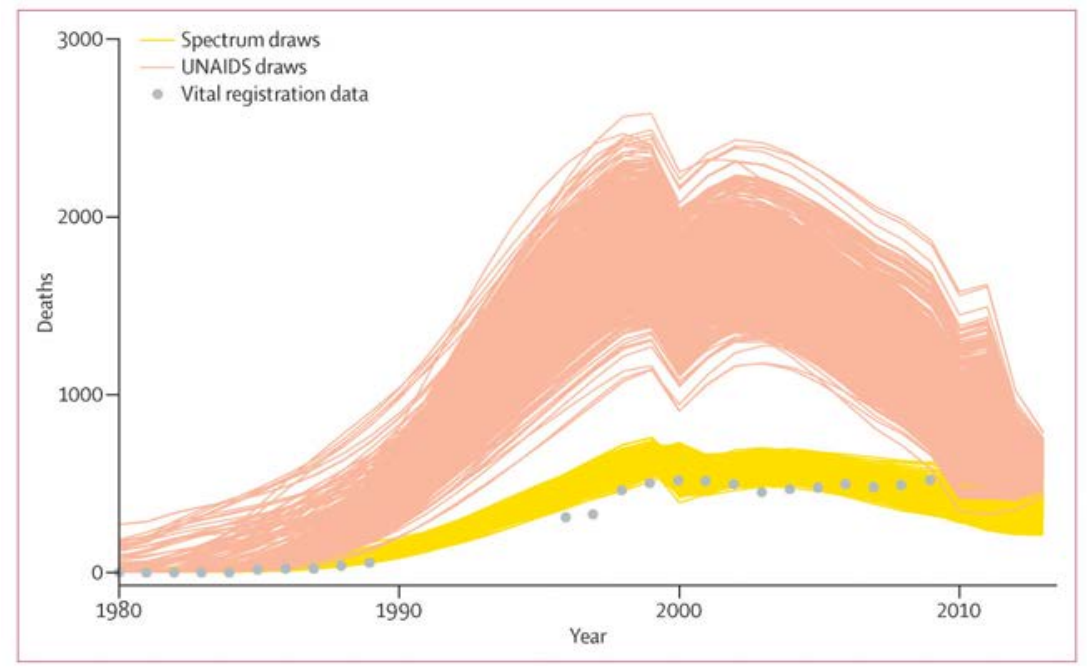

Figure 4. Vital registration inputs, and Spectrum and UNAIDS HIV mortality draws for Panama 

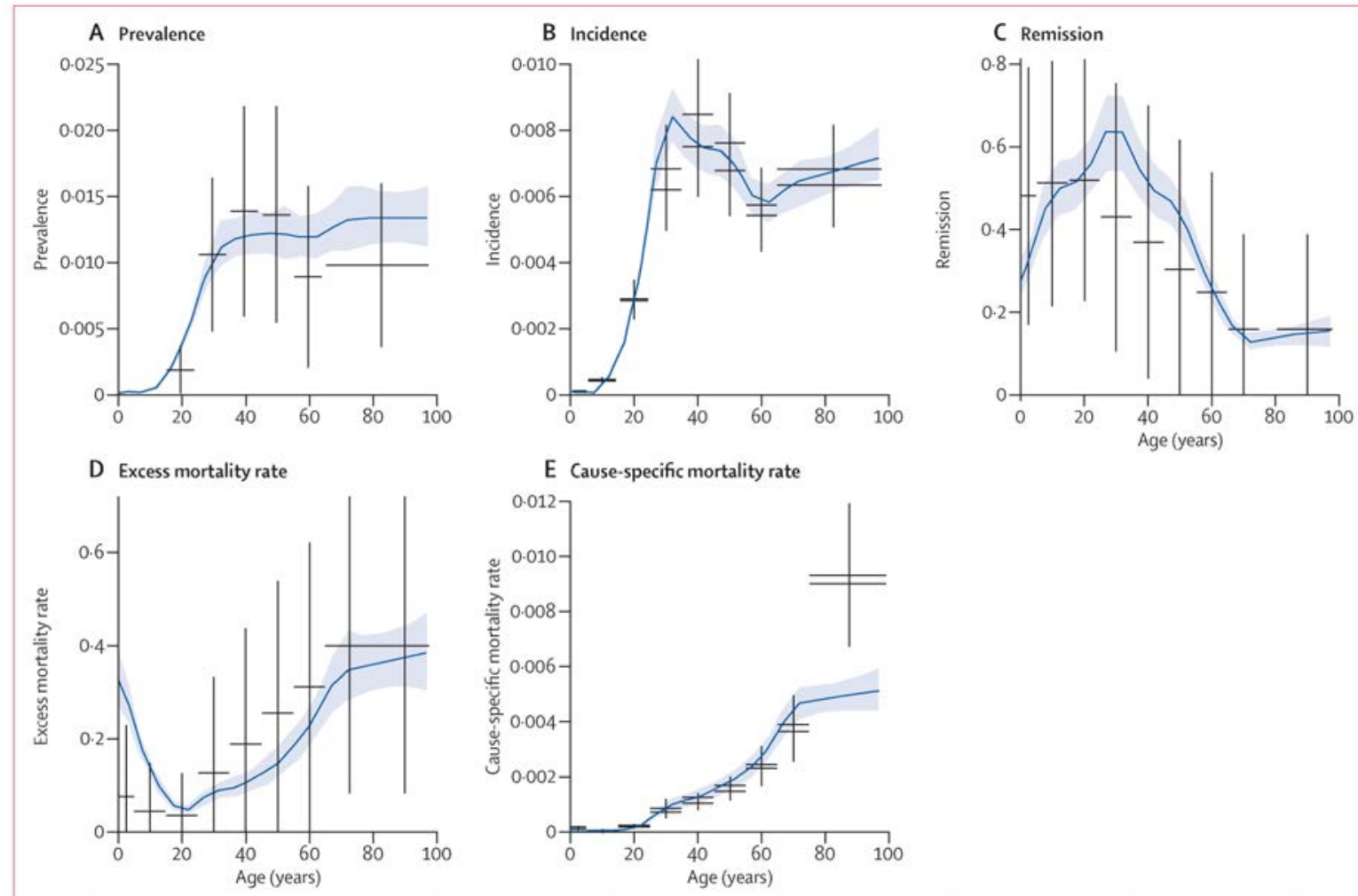

Figure 5. Bayesian meta-regression estimates for tuberculosis prevalence (A), incidence (B), remission (C), excess mortality (D), and cause-specific mortality (E) for male individuals in Kenya, 2013

For each observation in black, the length of the horizontal bar refers to the age interval of the observation and the length of the verticle bar refers to the uncertainty interval. To stabilise estimates, all data from 2008 to 2013 are included. 


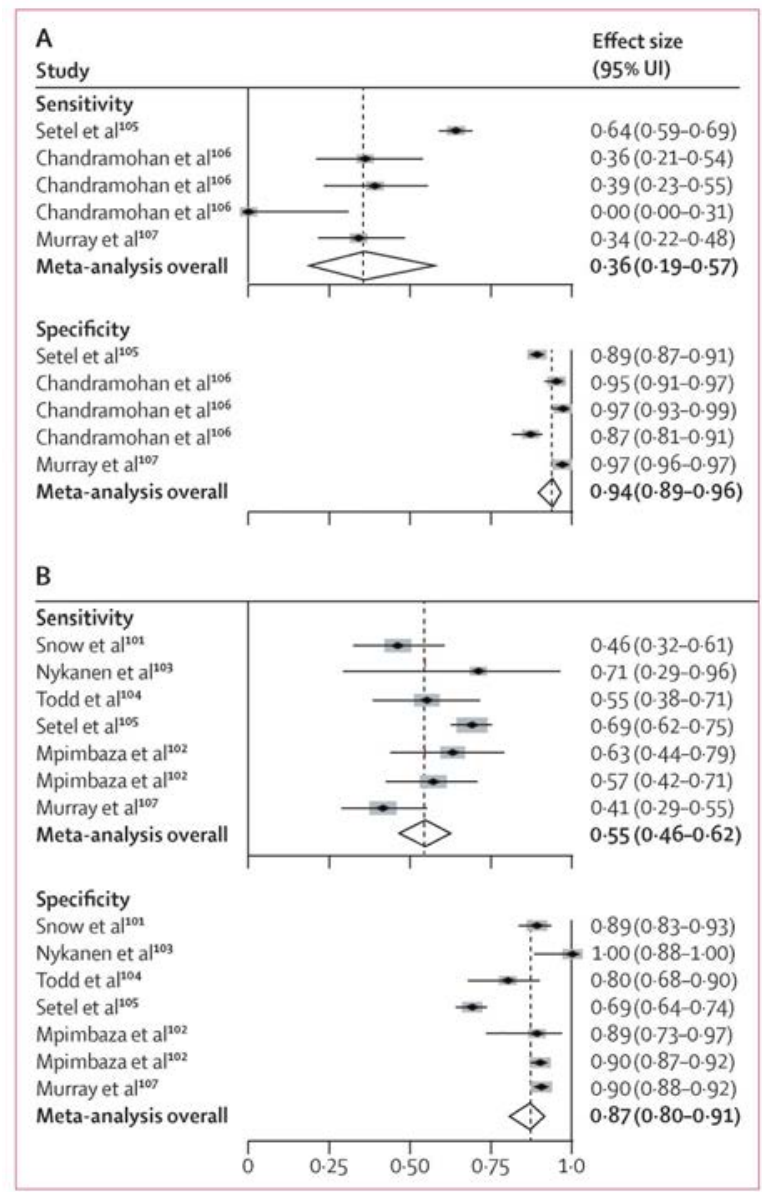

Figure 6. Meta-analysis of published validation studies of verbal autopsy for malaria in adults (A) and children (B), both sexes combined The solid lines show 95\% uncertainty intervals (ui); the dotted lines show the point estimate of the pooled estimate (the diamond). The age cutoff for children is $<15$ years. 


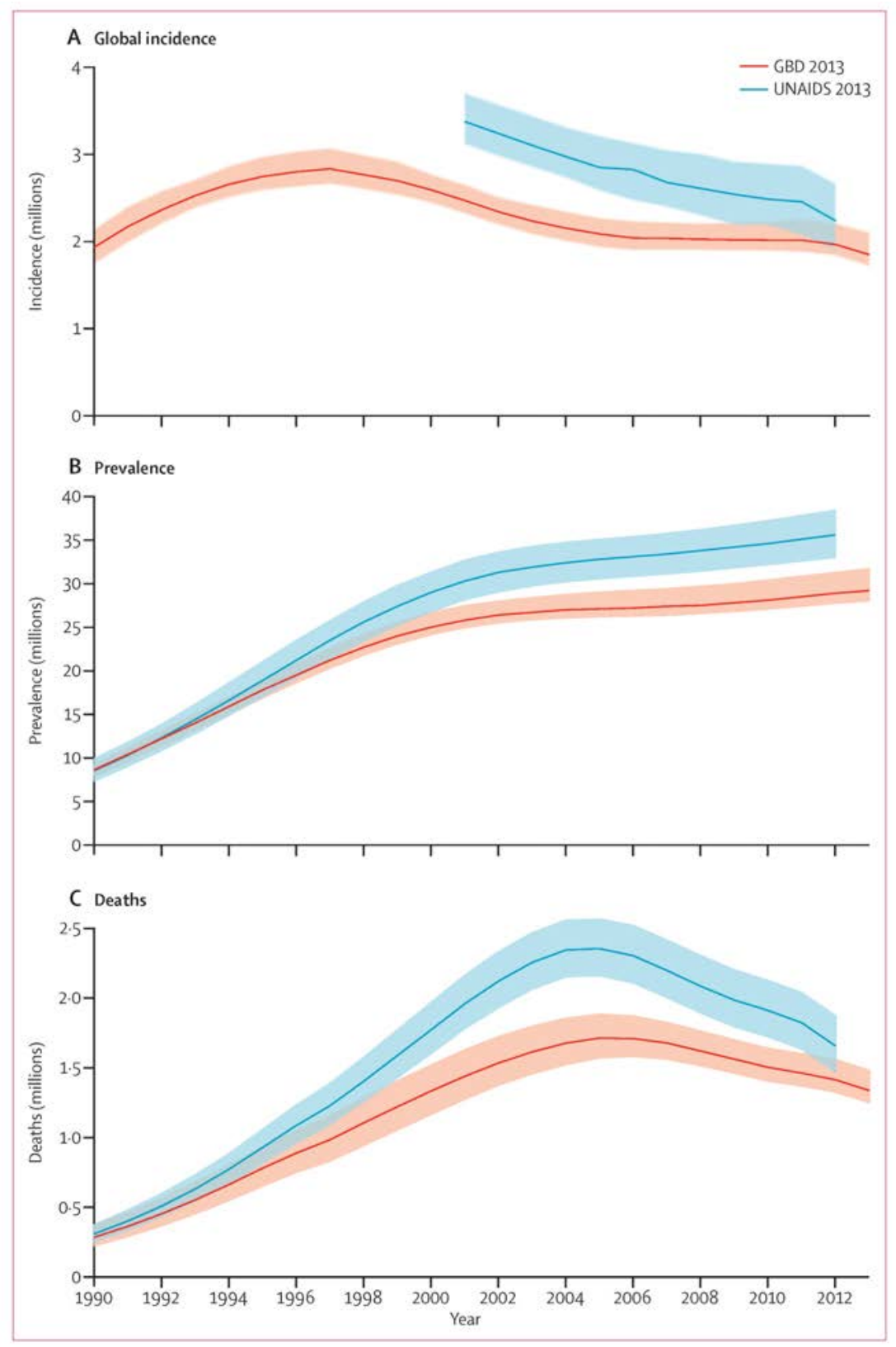

Figure 7. Global HIV incidence (A), prevalence (B), and mortality (C), 1990-2013, for all ages and both sexes combined

Shaded areas are $95 \%$ uncertainty intervals. 


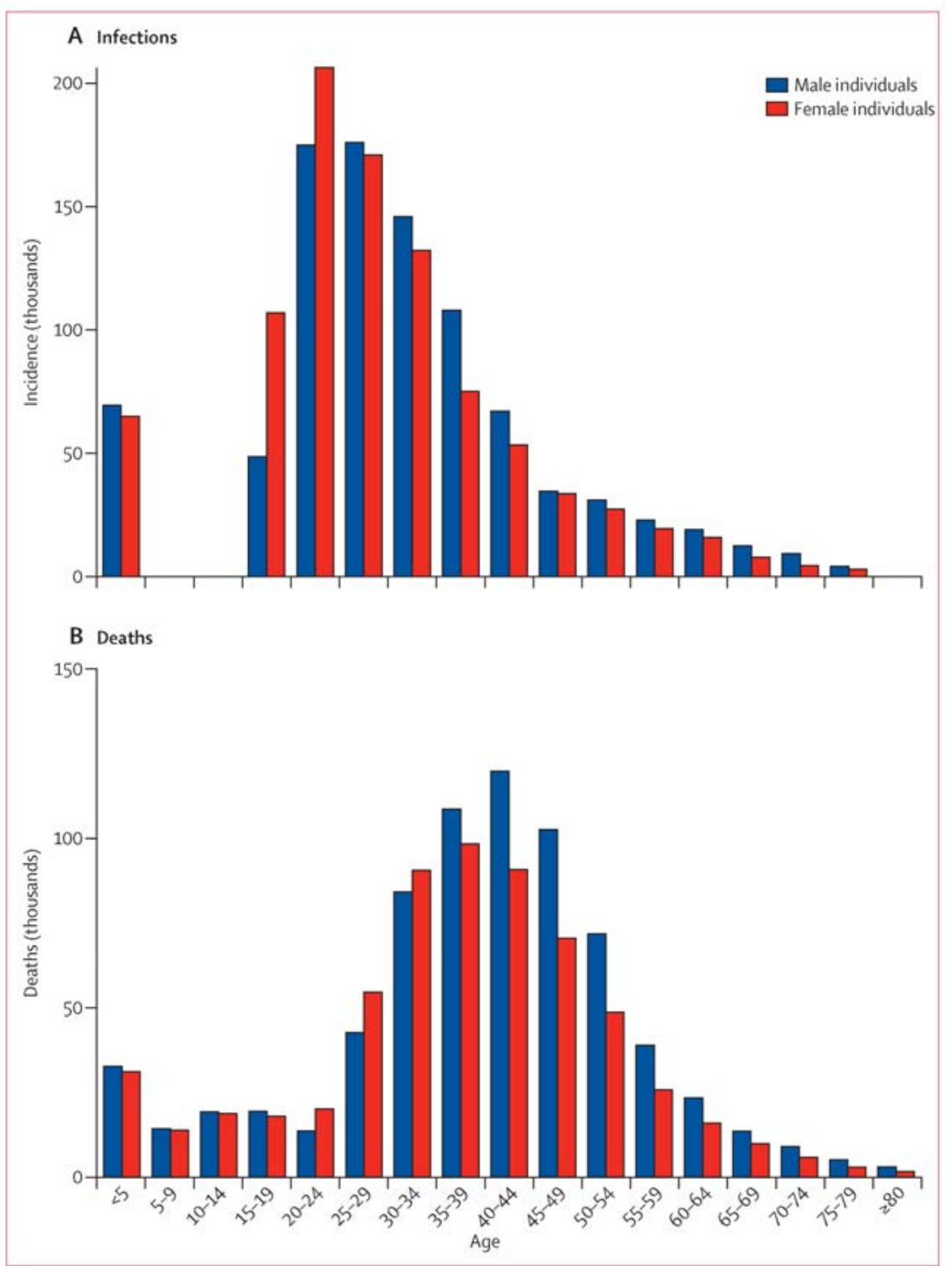

Figure 8. Global age-sex distribution of new HIV infections (A) and deaths (B) in 2013 


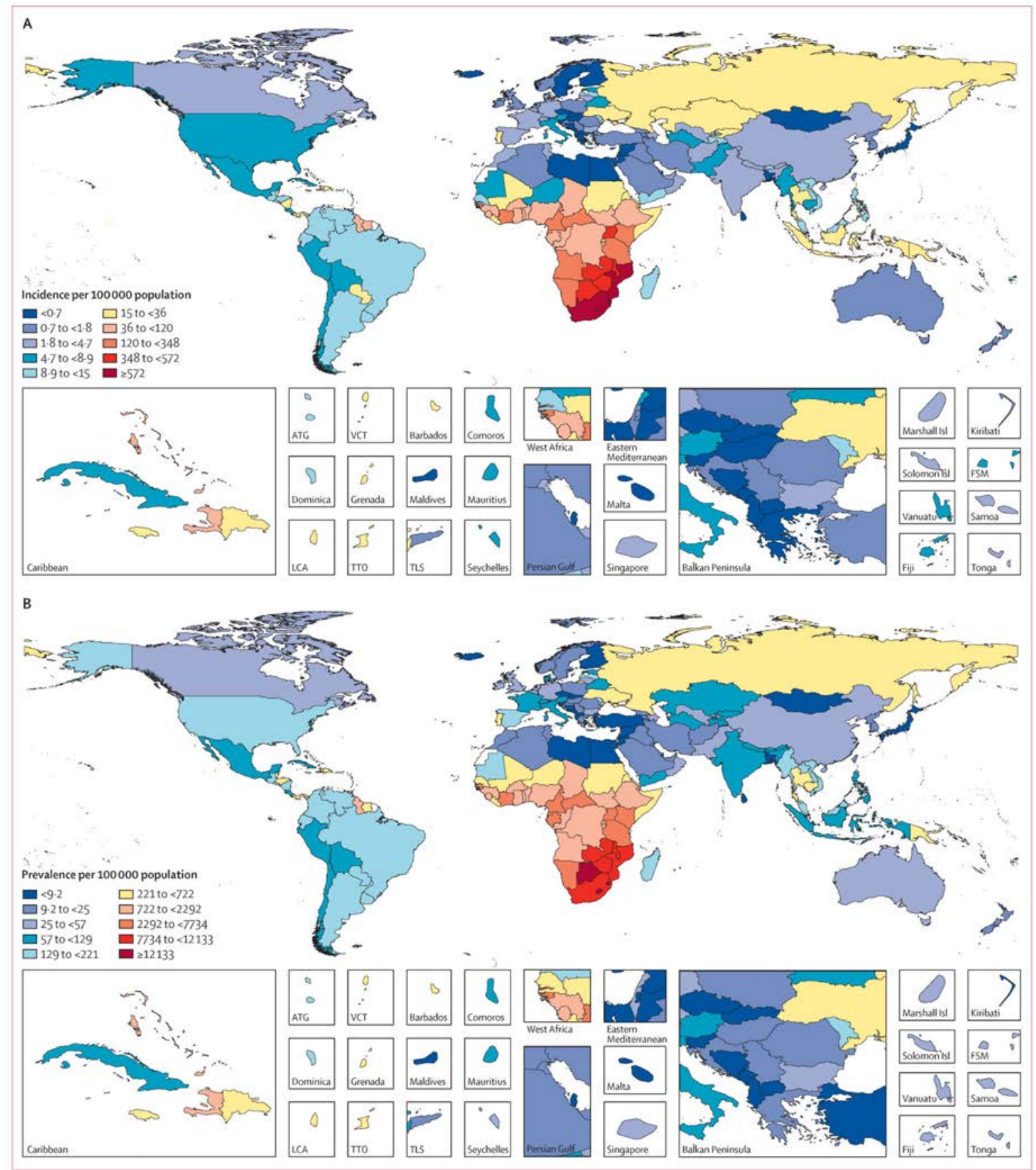

Figure 9. Age-standardised HIV incidence (A) and prevalence (B) in 2013, both sexes ATG=Antigua and Barbuda. VCT=Saint Vincent and the Grenadines. Isl=Islands. FSM=Federated States of Micronesia. LCA=Saint Lucia. TTO=Trinidad and Tobago. TLS=Timor-Leste 


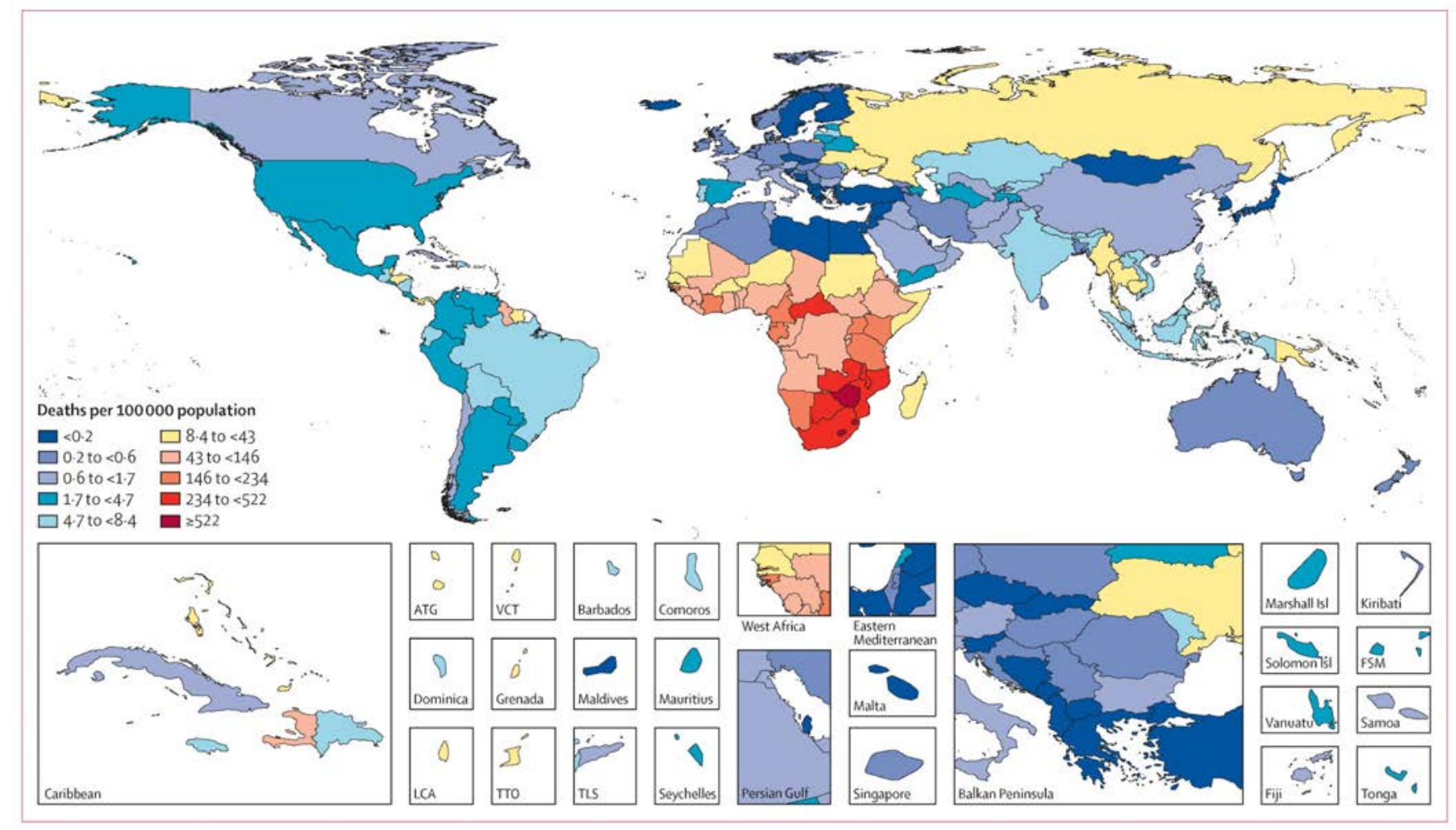

Figure 10. Age-standardised HIV mortality in 2013, both sexes

ATG=Antigua and Barbuda. VCT=Saint Vincent and the Grenadines. Isl=Islands.

FSM=Federated States of Micronesia. LCA=Saint Lucia. TTO=Trinidad and Tobago.

TLS=Timor-Leste. 


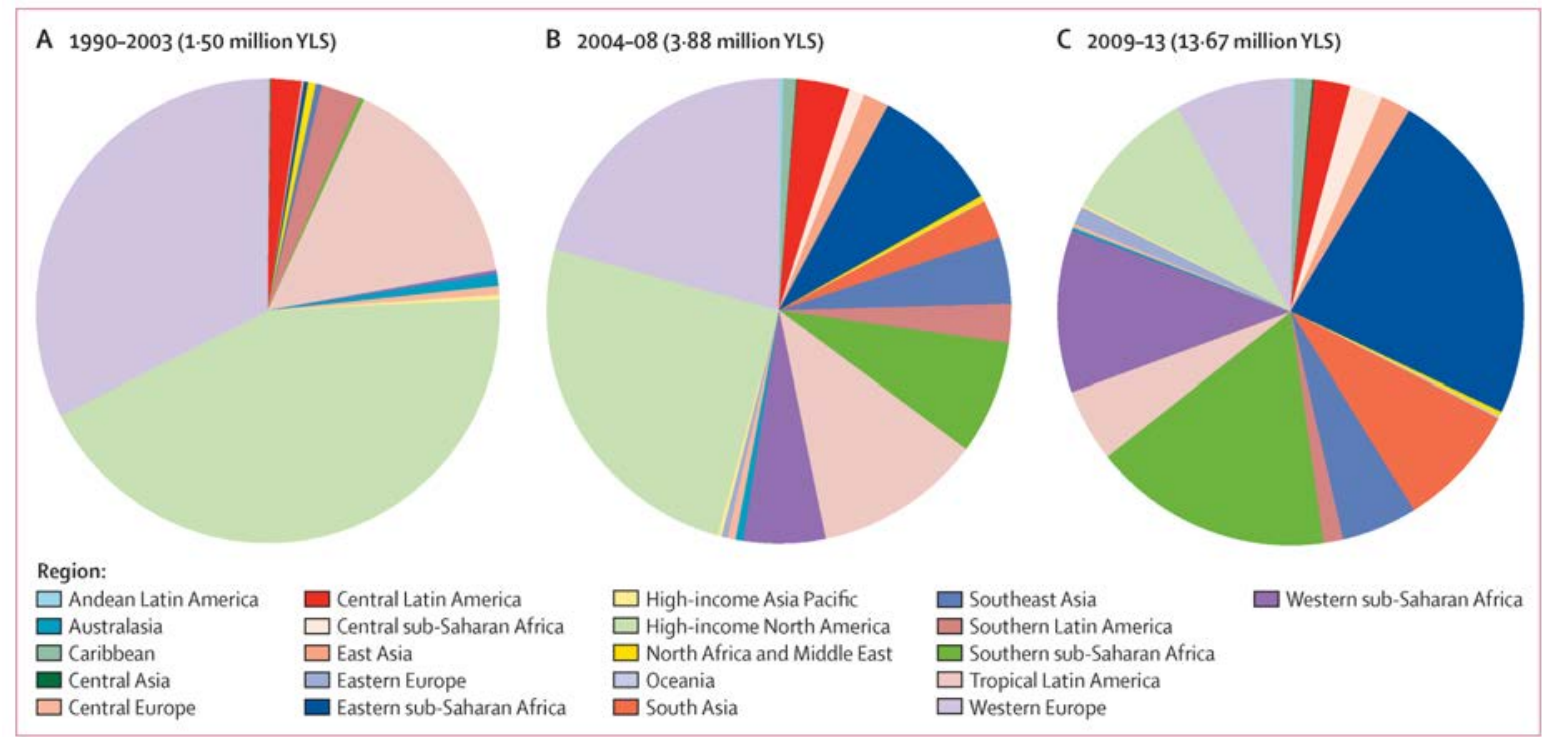

Figure 11. Years of life saved (YLS) through prophylactic treatment by region in 1990-2003 (A), 2004-08 (B), and 2009-13 (C), both sexes

Treatment includes antiretroviral therapy, prevention of mother-to-child transmission, and co-trimoxazole prophylaxis. 


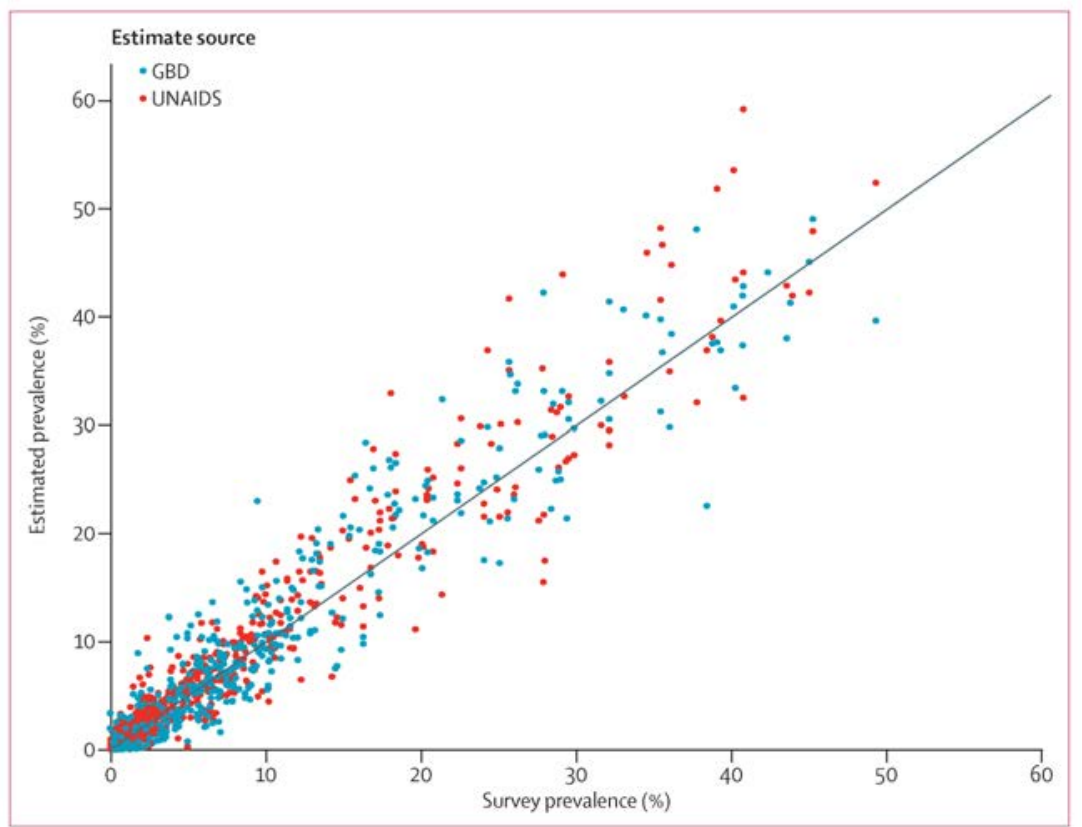

Figure 12. Comparison of 43 national HIV prevalence survey results for people aged 15-49 to prevalence estimates from the GBD 2013 and UNAIDS for the same year and age-group Each point corresponds to an estimate for a particular country, survey year, sex, and 5-year age group. The solid line indicates the line of equivalence. 


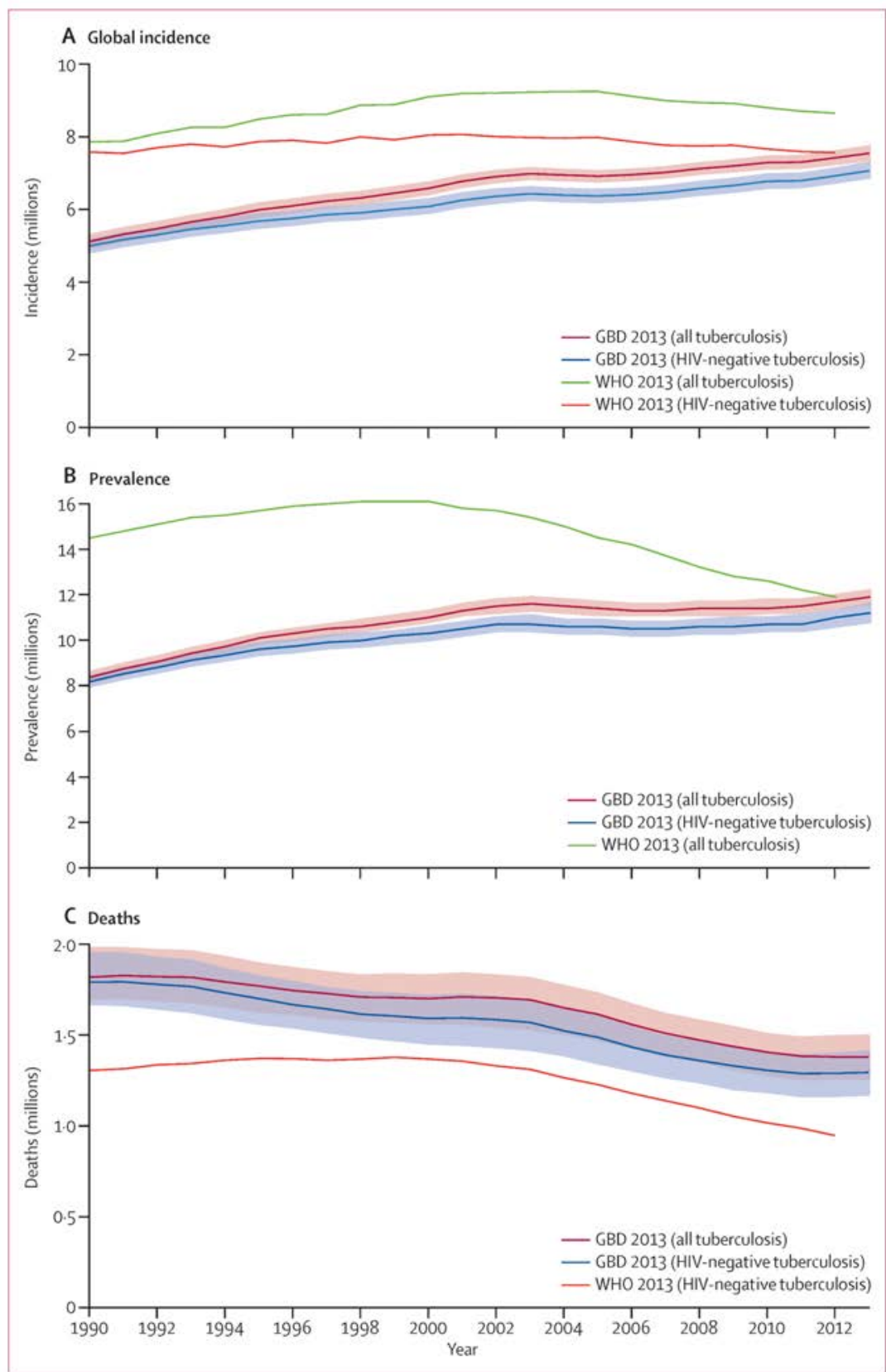

Figure 13. Global tuberculosis incidence (A), prevalence (B), and deaths (C), 1990-2013, for all ages and both sexes combined

Shaded areas show $95 \%$ uncertainty intervals. 


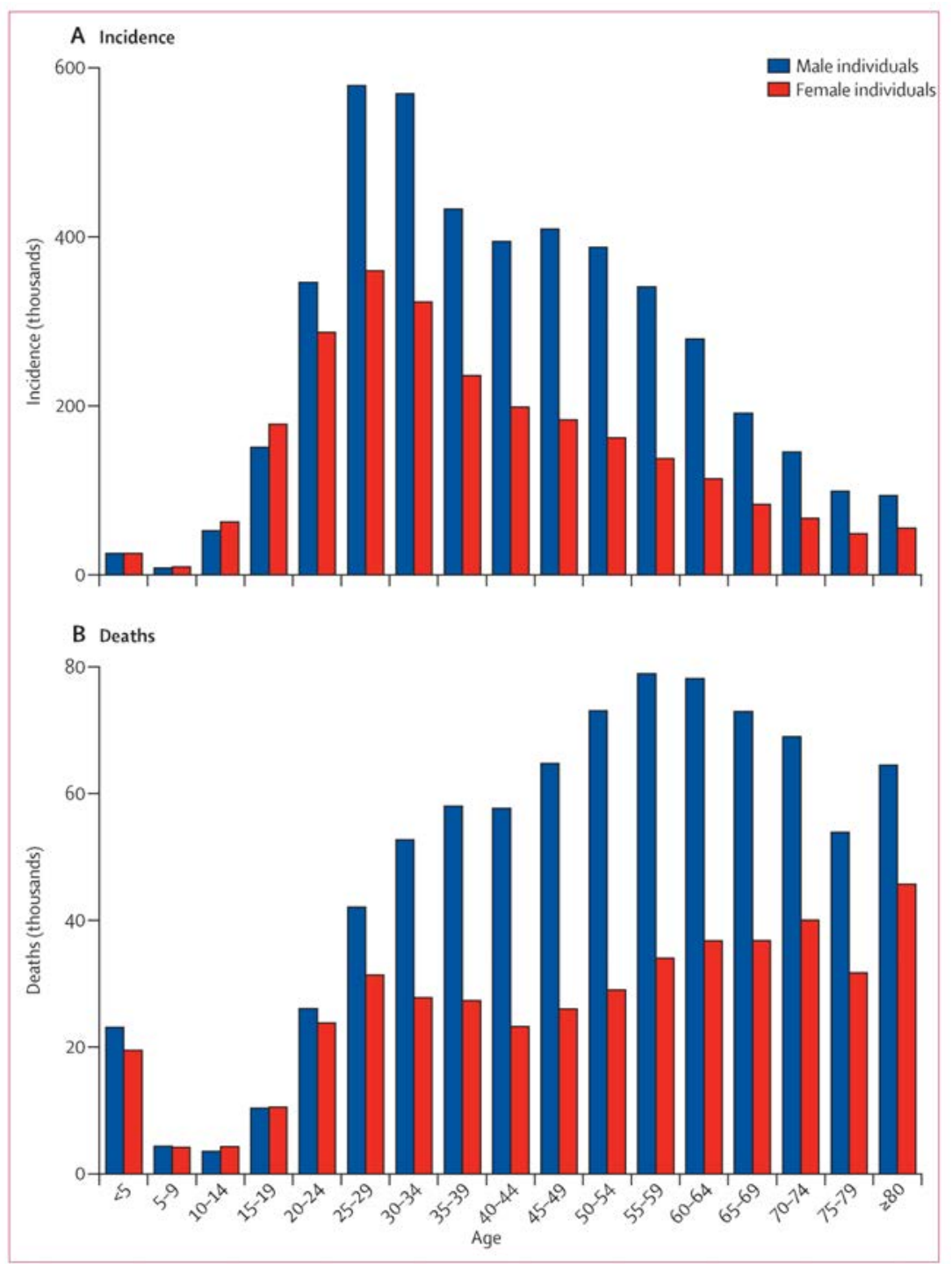

Figure 14. Global age-sex distribution of tuberculosis incidence (A) and deaths (B) in HIVnegative individuals in 2013 


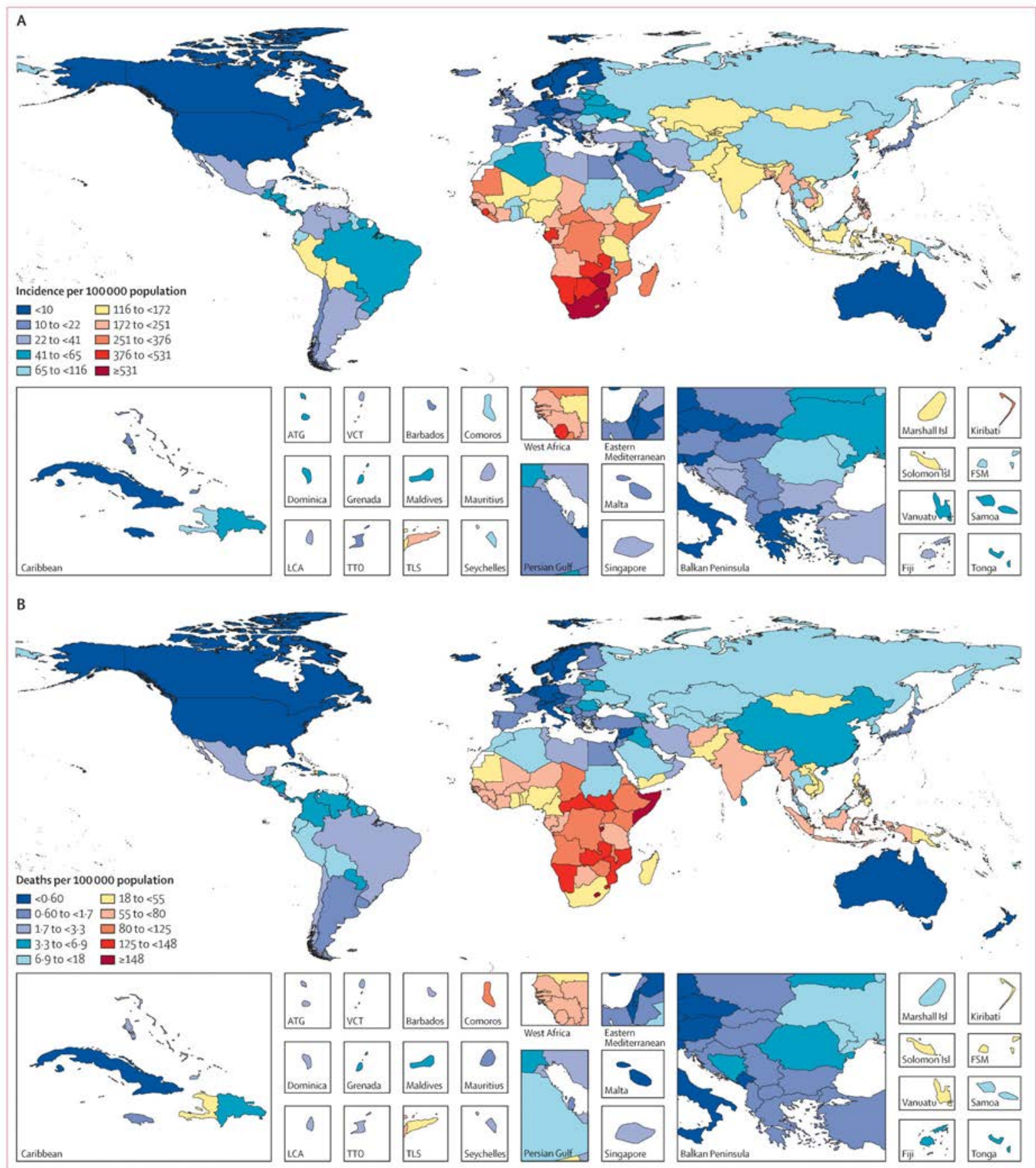

Figure 15. Age-standardised tuberculosis incidence (A) and death rates (B) in HIV-negative individuals in 2013, both sexes

ATG=Antigua and Barbuda. VCT=Saint Vincent and the Grenadines. Isl=Islands.

FSM=Federated States of Micronesia. LCA=Saint Lucia. TTO=Trinidad and Tobago.

TLS=Timor-Leste. 


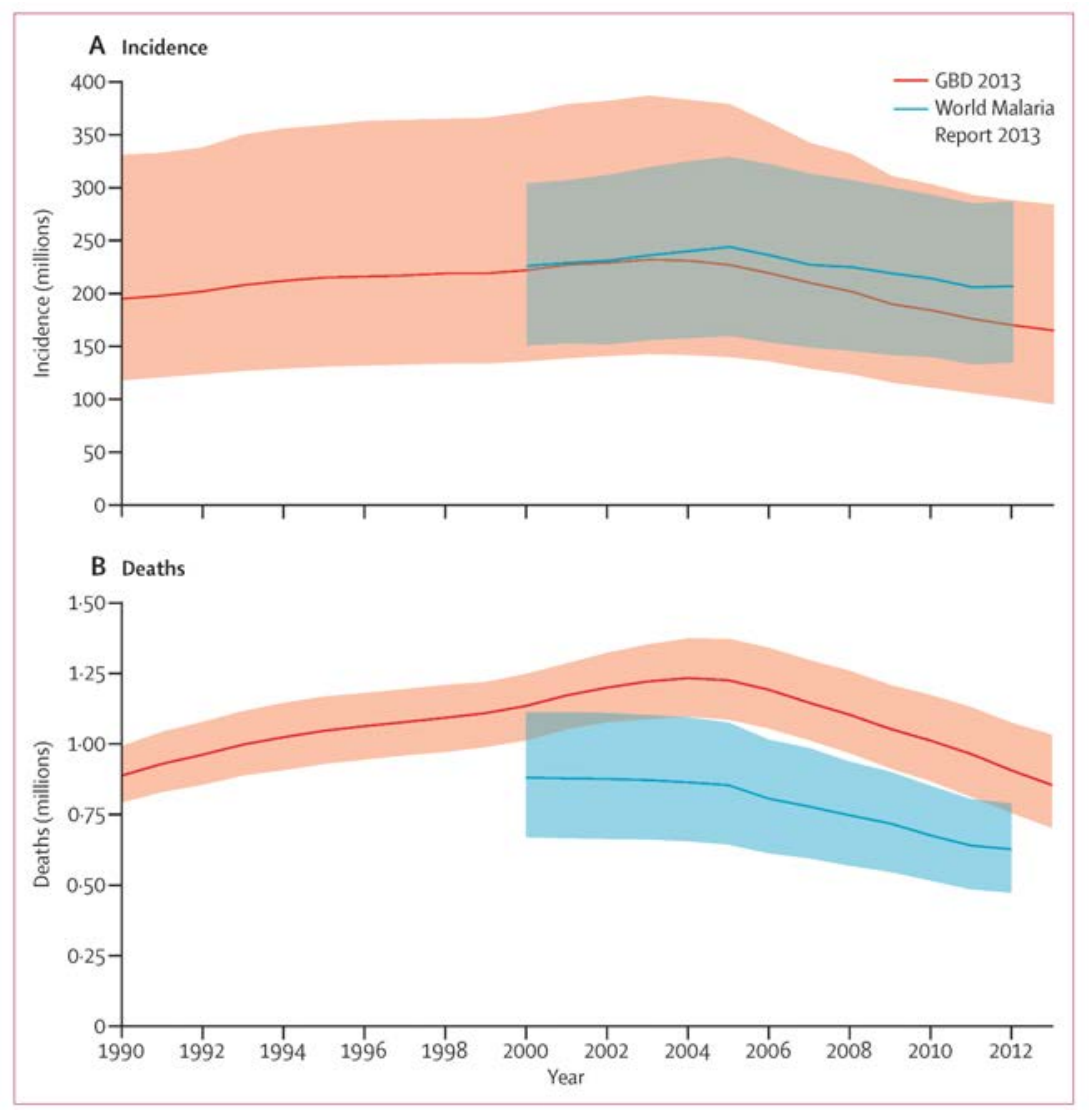

Figure 16. Global malaria incidence (A) and deaths (B), 1990-2013, for all ages and both sexes combined Shaded areas are $95 \%$ uncertainty intervals. 

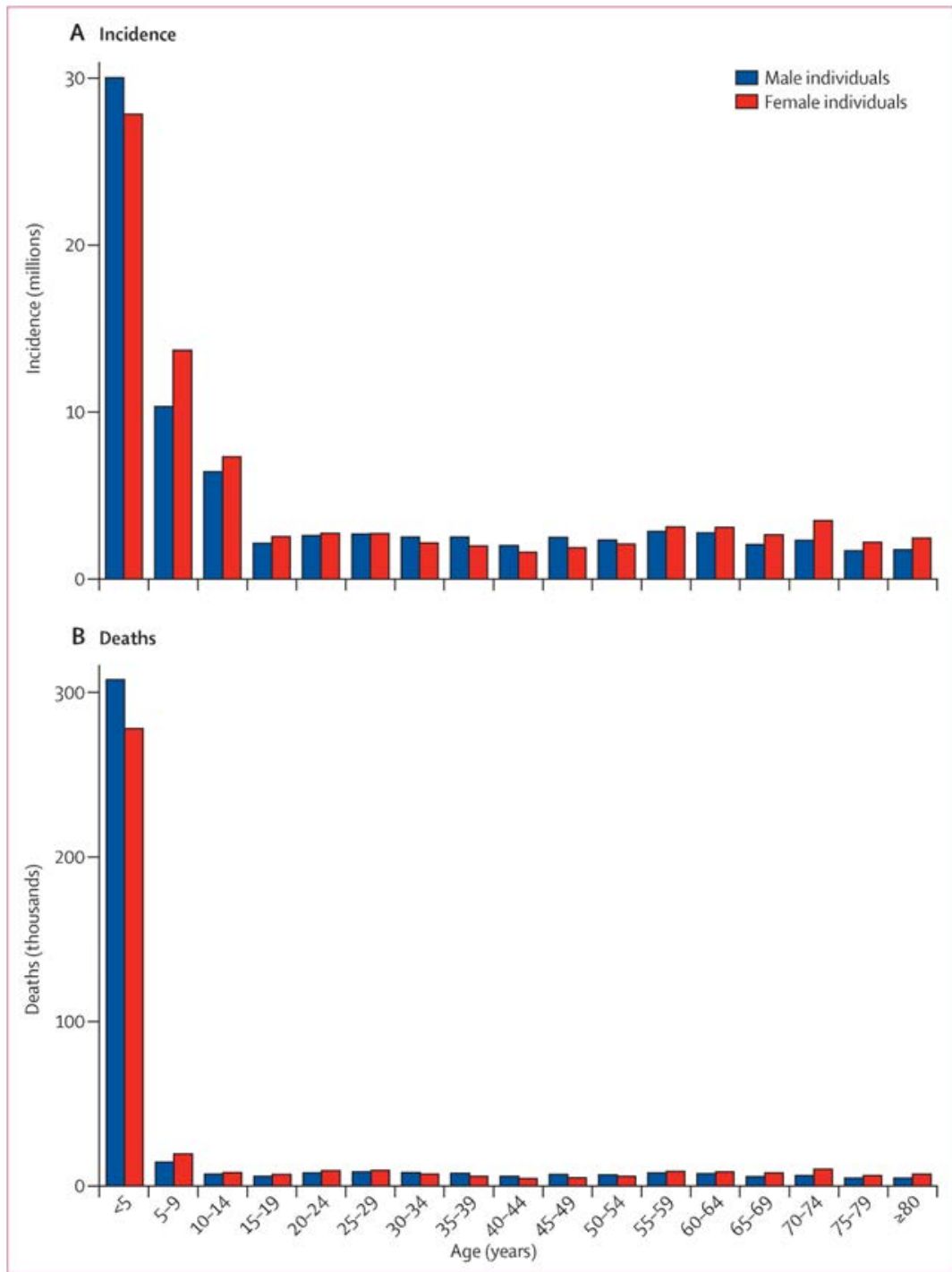

Figure 17. Global age-sex distribution of malaria incidence (A) and deaths (B) in 2013 


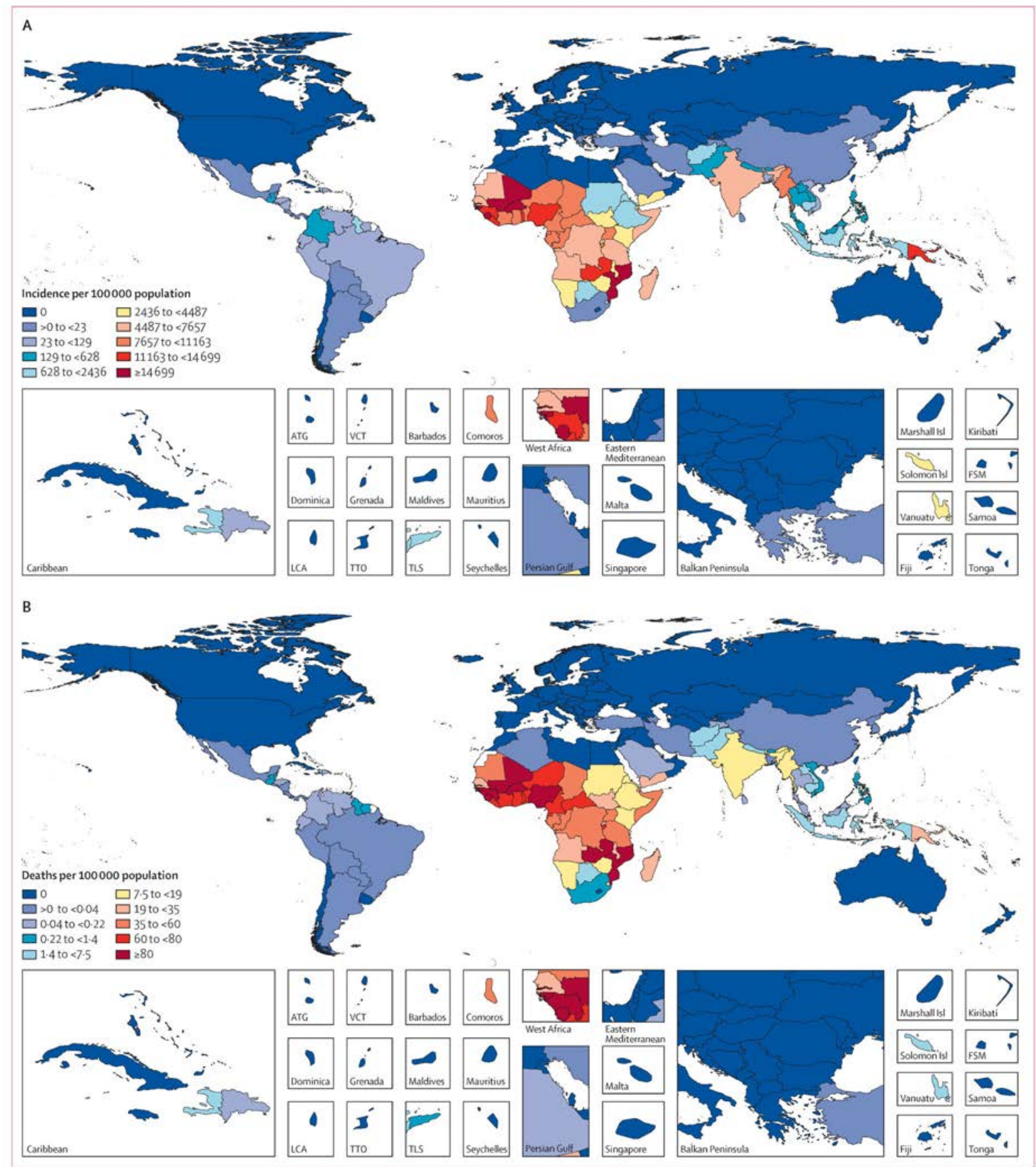

Figure 18. Age-standardised malaria incidence (A) and death (B) rates in 2013, both sexes ATG=Antigua and Barbuda. VCT=Saint Vincent and the Grenadines. Isl=Islands. FSM=Federated States of Micronesia. LCA=Saint Lucia. TTO=Trinidad and Tobago. TLS=Timor-Leste. 
Table 1

HIV-specific mortality rates for adults aged 25-34 years on antiretroviral therapy in subSaharan Africa, by time since initiation, sex, and initial CD4 count (per 100 person-years)

\begin{tabular}{|c|c|c|c|c|c|c|}
\hline & \multicolumn{3}{|c|}{ Women (time since initiation) } & \multicolumn{3}{|c|}{ Men (time since initiation) } \\
\hline & 0-6 months & 7-12 months & 13-24 months & 0-6 months & 7-12 months & 13-24 months \\
\hline$<50 \mathrm{CD} 4$ cells per $\mu \mathrm{L}$ & $40 \cdot 6(20 \cdot 4-71 \cdot 5)$ & $8 \cdot 9(6 \cdot 0-13 \cdot 4)$ & $4.0(2.7-5.7)$ & $53 \cdot 1(26 \cdot 5-93 \cdot 5)$ & $11 \cdot 6(7 \cdot 9-17 \cdot 5)$ & $5 \cdot 2(3 \cdot 6-7 \cdot 5)$ \\
\hline 50-99 CD4 cells per $\mu \mathrm{L}$ & $17 \cdot 4(12 \cdot 2-25 \cdot 0)$ & $6 \cdot 5(5 \cdot 0-8 \cdot 4)$ & $3 \cdot 0(2 \cdot 3-3 \cdot 9)$ & $22 \cdot 8(16 \cdot 2-32 \cdot 4)$ & $8 \cdot 6(6 \cdot 5-11 \cdot 2)$ & $3 \cdot 9(3 \cdot 0-5 \cdot 1)$ \\
\hline 100-199 CD4 cells per $\mu \mathrm{L}$ & $14 \cdot 1(10 \cdot 4-18 \cdot 4)$ & $5 \cdot 7(4 \cdot 4-7 \cdot 2)$ & $2 \cdot 6(2 \cdot 0-3 \cdot 3)$ & $18 \cdot 4(13 \cdot 6-24 \cdot 2)$ & $7 \cdot 4(5 \cdot 8-9 \cdot 5)$ & $3 \cdot 4(2 \cdot 6-4 \cdot 3)$ \\
\hline 200-249 CD4 cells per $\mu \mathrm{L}$ & $12 \cdot 5(8 \cdot 9-16 \cdot 6)$ & $5 \cdot 1(3 \cdot 8-6 \cdot 5)$ & $2 \cdot 4(1 \cdot 8-3 \cdot 1)$ & $16 \cdot 3(11 \cdot 6-21 \cdot 7)$ & $6 \cdot 6(4 \cdot 9-8 \cdot 6)$ & $3 \cdot 1(2 \cdot 3-4 \cdot 0)$ \\
\hline $250-349$ CD4 cells per $\mu \mathrm{L}$ & $10 \cdot 7(7 \cdot 0-14 \cdot 8)$ & $4 \cdot 4(3 \cdot 0-6 \cdot 0)$ & $2 \cdot 0(1 \cdot 4-2 \cdot 7)$ & $14 \cdot 0(9 \cdot 3-19 \cdot 4)$ & $5 \cdot 7(3 \cdot 9-7 \cdot 8)$ & $2 \cdot 6(1 \cdot 8-3 \cdot 6)$ \\
\hline 350-499 CD4 cells per $\mu \mathrm{L}$ & $8 \cdot 1(4 \cdot 7-12 \cdot 0)$ & $3 \cdot 1(1 \cdot 8-4 \cdot 8)$ & $1.4(0 \cdot 7-2 \cdot 2)$ & $10 \cdot 5(6 \cdot 0-15 \cdot 8)$ & $4 \cdot 1(2 \cdot 4-6 \cdot 2)$ & $1 \cdot 8(1 \cdot 0-2 \cdot 8)$ \\
\hline $2500 \mathrm{CD} 4$ cells per $\mu \mathrm{L}$ & $6 \cdot 3(0 \cdot 9-11 \cdot 0)$ & $2 \cdot 3(0 \cdot 1-4 \cdot 4)$ & $0 \cdot 9(0 \cdot 0-2 \cdot 0)$ & $8 \cdot 2(1 \cdot 2-14 \cdot 7)$ & $3 \cdot 0(0 \cdot 1-5 \cdot 7)$ & $1 \cdot 2(0 \cdot 0-2 \cdot 6)$ \\
\hline
\end{tabular}

Data in parentheses are $95 \%$ uncertainty intervals. 
Table 2

Age-standardised HIV/AIDS incidence, prevalence, and mortality rates, and annualised rates of change for both sexes for 21 Global Burden of Disease regions

\begin{tabular}{|c|c|c|c|c|c|c|c|c|c|}
\hline & \multicolumn{3}{|c|}{$\begin{array}{l}\text { Age-standardised rates in } 2013 \text { (per } \\
100000 \text { population) }\end{array}$} & \multicolumn{6}{|c|}{ Annualised rate of change (\%) } \\
\hline & & & & 1990-2000 & & & 2000-2013 & & \\
\hline & Incidence & Prevalence & Mortality & Incidence & Prevalence & Mortality & Incidence & Prevalence & Mortality \\
\hline Worldwide & $\begin{array}{l}24 \cdot 84 \\
(23 \cdot 10 \text { to } \\
28 \cdot 26)\end{array}$ & $\begin{array}{c}400 \cdot 98 \\
(385 \cdot 51 \text { to } \\
435 \cdot 10)\end{array}$ & $\begin{array}{c}18 \cdot 51 \\
(17 \cdot 38 \text { to } \\
20 \cdot 46)\end{array}$ & $\begin{array}{l}1 \cdot 27(0 \cdot 24 \\
\text { to } 2 \cdot 16)\end{array}$ & $\begin{array}{l}8 \cdot 87(8 \cdot 36 \\
\text { to } 9 \cdot 52)\end{array}$ & $\begin{array}{l}13.62 \\
(11.93 \text { to } \\
15 \cdot 38)\end{array}$ & $\begin{array}{l}-3 \cdot 92 \\
(-4 \cdot 50 \text { to } \\
-3 \cdot 15)\end{array}$ & $\begin{array}{l}-0 \cdot 20 \\
(-0.42 \text { to } \\
0 \cdot 05)\end{array}$ & $\begin{array}{l}-1 \cdot 54 \\
(-2 \cdot 36 \text { to } \\
-0.59)\end{array}$ \\
\hline High-income Asia Pacific & $\begin{array}{l}0 \cdot 49(0 \cdot 28 \\
\text { to } 0 \cdot 70)\end{array}$ & $\begin{array}{l}7 \cdot 22(4 \cdot 81 \\
\text { to } 9 \cdot 97)\end{array}$ & $\begin{array}{l}0 \cdot 10(0 \cdot 08 \\
\text { to } 0 \cdot 13)\end{array}$ & $\begin{array}{l}7 \cdot 74(3 \cdot 65 \\
\text { to } 13 \cdot 26)\end{array}$ & $\begin{array}{l}6 \cdot 94(5 \cdot 12 \\
\text { to } 9 \cdot 46)\end{array}$ & $\begin{array}{l}0.77 \\
(-1 \cdot 11 \text { to } \\
2 \cdot 92)\end{array}$ & $\begin{array}{l}-1 \cdot 55 \\
(-5 \cdot 91 \text { to } \\
1 \cdot 57)\end{array}$ & $\begin{array}{l}6 \cdot 29(4 \cdot 49 \\
\text { to } 8 \cdot 22)\end{array}$ & $\begin{array}{l}1 \cdot 13 \\
(-0 \cdot 55 \text { to } \\
3 \cdot 20)\end{array}$ \\
\hline Central Asia & $\begin{array}{l}7 \cdot 02(3 \cdot 62 \\
\text { to } 15 \cdot 56)\end{array}$ & $\begin{array}{c}61.98 \\
(38.09 \text { to } \\
105 \cdot 12)\end{array}$ & $\begin{array}{l}2 \cdot 67(1 \cdot 52 \\
\text { to } 4 \cdot 08)\end{array}$ & $\begin{array}{l}8 \cdot 17 \\
(-3.90 \text { to } \\
19 \cdot 78)\end{array}$ & $\begin{array}{l}13 \cdot 11(1 \cdot 09 \\
\text { to } 24 \cdot 09)\end{array}$ & $\begin{array}{l}21 \cdot 91 \\
(9 \cdot 43 \text { to } \\
33 \cdot 39)\end{array}$ & $\begin{array}{l}4 \cdot 18 \\
(-2 \cdot 22 \text { to } \\
12 \cdot 58)\end{array}$ & $\begin{array}{l}5 \cdot 46(1 \cdot 76 \\
\text { to } 9 \cdot 62)\end{array}$ & $\begin{array}{l}2 \cdot 65 \\
(-2 \cdot 01 \text { to } \\
5 \cdot 83)\end{array}$ \\
\hline East Asia & $\begin{array}{l}2 \cdot 28(1 \cdot 31 \\
\text { to } 4 \cdot 09)\end{array}$ & $\begin{array}{c}26 \cdot 37 \\
(14 \cdot 36 \text { to } \\
41 \cdot 32)\end{array}$ & $\begin{array}{l}0.77(0.60 \\
\text { to } 1.05)\end{array}$ & $\begin{array}{l}38 \cdot 86 \\
(27 \cdot 75 \text { to } \\
62 \cdot 64)\end{array}$ & $\begin{array}{l}41 \cdot 29 \\
(30 \cdot 82 \text { to } \\
62 \cdot 45)\end{array}$ & $\begin{array}{l}45 \cdot 26 \\
(29 \cdot 11 \text { to } \\
70 \cdot 18)\end{array}$ & $\begin{array}{l}1 \cdot 41 \\
(-3 \cdot 60 \text { to } \\
6 \cdot 83)\end{array}$ & $\begin{array}{l}8 \cdot 07(4.79 \\
\text { to } 11 \cdot 46)\end{array}$ & $\begin{array}{l}6 \cdot 09(2 \cdot 91 \\
\text { to } 10 \cdot 38)\end{array}$ \\
\hline South Asia & $\begin{array}{l}2 \cdot 69(0 \cdot 85 \\
\text { to } 5 \cdot 51)\end{array}$ & $\begin{array}{c}93.97 \\
(84.36 \text { to } \\
112.06)\end{array}$ & $\begin{array}{l}5 \cdot 42(4 \cdot 43 \\
\text { to } 7 \cdot 18)\end{array}$ & $\begin{array}{l}-9 \cdot 72 \\
(-15 \cdot 85 \\
\text { to }-5 \cdot 81)\end{array}$ & $\begin{array}{l}3 \cdot 91(1 \cdot 89 \\
\text { to } 5 \cdot 98)\end{array}$ & $\begin{array}{l}13 \cdot 67 \\
(7 \cdot 68 \text { to } \\
23 \cdot 47)\end{array}$ & $\begin{array}{l}-13 \cdot 93 \\
(-19 \cdot 71 \\
\text { to }-8 \cdot 09)\end{array}$ & $\begin{array}{l}-7 \cdot 85 \\
(-8.67 \text { to } \\
-6.64)\end{array}$ & $\begin{array}{l}-11 \cdot 58 \\
(-14 \cdot 45 \\
\text { to }-7 \cdot 11)\end{array}$ \\
\hline Southeast Asia & $\begin{array}{l}13 \cdot 88 \\
(6 \cdot 66 \text { to } \\
41 \cdot 04)\end{array}$ & $\begin{array}{c}176 \cdot 39 \\
(113 \cdot 22 \text { to } \\
421.49)\end{array}$ & $\begin{array}{l}7 \cdot 54(3 \cdot 90 \\
\text { to } 23 \cdot 55)\end{array}$ & $\begin{array}{l}-10 \cdot 46 \\
(-15 \cdot 54 \\
\text { to }-4 \cdot 52)\end{array}$ & $\begin{array}{l}8 \cdot 07(5 \cdot 78 \\
\text { to } 12 \cdot 44)\end{array}$ & $\begin{array}{l}29 \cdot 51 \\
(25 \cdot 47 \text { to } \\
33.97)\end{array}$ & $\begin{array}{l}1 \cdot 29 \\
(-2 \cdot 74 \text { to } \\
7 \cdot 36)\end{array}$ & $\begin{array}{l}0 \cdot 97(-0 \cdot 78 \\
\text { to } 3 \cdot 51)\end{array}$ & $\begin{array}{l}-3 \cdot 87 \\
(-6 \cdot 62 \text { to } \\
1 \cdot 36)\end{array}$ \\
\hline Australasia & $\begin{array}{l}1 \cdot 50(0 \cdot 81 \\
\text { to } 2 \cdot 32)\end{array}$ & $\begin{array}{c}35.09 \\
(22 \cdot 80 \text { to } \\
50 \cdot 24)\end{array}$ & $\begin{array}{l}0.37(0.26 \\
\text { to } 0.53)\end{array}$ & $\begin{array}{l}-7 \cdot 07 \\
(-11 \cdot 53 \\
\text { to }-3 \cdot 51)\end{array}$ & $\begin{array}{l}-1.86 \\
(-3.67 \text { to } \\
-0.18)\end{array}$ & $\begin{array}{l}-11 \cdot 88 \\
(-14 \cdot 40 \\
\text { to }-9 \cdot 10)\end{array}$ & $\begin{array}{l}0 \cdot 41 \\
(-1 \cdot 27 \text { to } \\
2 \cdot 85)\end{array}$ & $\begin{array}{l}1 \cdot 07(-0.53 \\
\text { to } 2 \cdot 49)\end{array}$ & $\begin{array}{l}-4 \cdot 51 \\
(-6 \cdot 49 \text { to } \\
-2 \cdot 60)\end{array}$ \\
\hline Central Europe & $\begin{array}{l}0.95(0.71 \\
\text { to } 1.23)\end{array}$ & $\begin{array}{c}16 \cdot 62 \\
(13 \cdot 41 \text { to } \\
20 \cdot 11)\end{array}$ & $\begin{array}{l}0.44(0.37 \\
\text { to } 0.51)\end{array}$ & $\begin{array}{l}1.29 \\
(-1.78 \text { to } \\
4.40)\end{array}$ & $\begin{array}{l}8.90(6 \cdot 61 \\
\text { to } 11 \cdot 36)\end{array}$ & $\begin{array}{l}8.06(6 \cdot 44 \\
\text { to } 10 \cdot 09)\end{array}$ & $\begin{array}{l}-0 \cdot 55 \\
(-3.44 \text { to } \\
2 \cdot 08)\end{array}$ & $\begin{array}{l}2 \cdot 37(1 \cdot 20 \\
\text { to } 3 \cdot 44)\end{array}$ & $\begin{array}{l}0 \cdot 19 \\
(-1 \cdot 28 \text { to } \\
1.41)\end{array}$ \\
\hline Eastern Europe & $\begin{array}{l}22 \cdot 58 \\
(16.56 \text { to } \\
31 \cdot 65)\end{array}$ & $\begin{array}{c}263.86 \\
(209 \cdot 53 \text { to } \\
347 \cdot 22)\end{array}$ & $\begin{array}{c}11 \cdot 14 \\
(8 \cdot 75 \text { to } \\
14 \cdot 14)\end{array}$ & $\begin{array}{l}25 \cdot 17 \\
(18 \cdot 06 \text { to } \\
34 \cdot 84)\end{array}$ & $\begin{array}{l}18 \cdot 47 \\
(14.57 \text { to } \\
24 \cdot 08)\end{array}$ & $\begin{array}{l}13 \cdot 07 \\
(9 \cdot 88 \text { to } \\
16 \cdot 41)\end{array}$ & $\begin{array}{l}-1.53 \\
(-3.75 \text { to } \\
0 \cdot 86)\end{array}$ & $\begin{array}{l}4 \cdot 75(3 \cdot 18 \\
\text { to } 6 \cdot 14)\end{array}$ & $\begin{array}{l}8 \cdot 51(6 \cdot 41 \\
\text { to } 10 \cdot 74)\end{array}$ \\
\hline Western Europe & $\begin{array}{l}3 \cdot 26(2 \cdot 56 \\
\text { to } 4 \cdot 22)\end{array}$ & $\begin{array}{c}80.97 \\
(66.16 \text { to } \\
99.08)\end{array}$ & $\begin{array}{l}1.06(0.90 \\
\text { to } 1.24)\end{array}$ & $\begin{array}{l}-9 \cdot 15 \\
(-14 \cdot 34 \\
\text { to }-5 \cdot 01)\end{array}$ & $\begin{array}{l}0 \cdot 92(-0 \cdot 14 \\
\text { to } 2 \cdot 04)\end{array}$ & $\begin{array}{l}-6 \cdot 75 \\
(-8 \cdot 06 \text { to } \\
-5 \cdot 34)\end{array}$ & $\begin{array}{l}0 \cdot 05 \\
(-1 \cdot 77 \text { to } \\
2 \cdot 05)\end{array}$ & $\begin{array}{l}0 \cdot 60(-0.21 \\
\text { to } 1 \cdot 62)\end{array}$ & $\begin{array}{l}-3 \cdot 59 \\
(-4 \cdot 72 \text { to } \\
-2 \cdot 25)\end{array}$ \\
\hline Andean Latin America & $\begin{array}{l}7 \cdot 96(4 \cdot 64 \\
\text { to } 16 \cdot 84)\end{array}$ & $\begin{array}{c}106 \cdot 52 \\
(76 \cdot 06 \text { to } \\
171 \cdot 80)\end{array}$ & $\begin{array}{l}3.92(2.40 \\
\text { to } 9 \cdot 39)\end{array}$ & $\begin{array}{l}1 \cdot 91 \\
(-16 \cdot 44 \\
\text { to } 14 \cdot 95)\end{array}$ & $\begin{array}{l}11 \cdot 70(2 \cdot 21 \\
\text { to } 21 \cdot 36)\end{array}$ & $\begin{array}{l}19.73 \\
(11.89 \text { to } \\
28.79)\end{array}$ & $\begin{array}{l}-1 \cdot 09 \\
(-6 \cdot 07 \text { to } \\
4 \cdot 63)\end{array}$ & $\begin{array}{l}1 \cdot 15(-1 \cdot 96 \\
\text { to } 4 \cdot 60)\end{array}$ & $\begin{array}{l}-3 \cdot 52 \\
(-6 \cdot 58 \text { to } \\
0 \cdot 62)\end{array}$ \\
\hline Central Latin America & $\begin{array}{l}9.43(7.48 \\
\text { to } 11.55)\end{array}$ & $\begin{array}{c}139 \cdot 92 \\
(118 \cdot 12 \text { to } \\
167 \cdot 80)\end{array}$ & $\begin{array}{l}4 \cdot 08(3 \cdot 13 \\
\text { to } 6 \cdot 16)\end{array}$ & $\begin{array}{l}-2 \cdot 00 \\
(-8 \cdot 23 \text { to } \\
1.40)\end{array}$ & $\begin{array}{l}7 \cdot 04(3 \cdot 66 \\
\text { to } 9 \cdot 32)\end{array}$ & $\begin{array}{l}13 \cdot 13 \\
(10 \cdot 27 \text { to } \\
16 \cdot 05)\end{array}$ & $\begin{array}{l}-0 \cdot 64 \\
(-1 \cdot 81 \text { to } \\
0 \cdot 37)\end{array}$ & $\begin{array}{l}0 \cdot 99(-1 \cdot 33 \\
\text { to } 2 \cdot 31)\end{array}$ & $\begin{array}{l}-5 \cdot 46 \\
(-7 \cdot 10 \text { to } \\
-3 \cdot 86)\end{array}$ \\
\hline
\end{tabular}




\begin{tabular}{|c|c|c|c|c|c|c|c|c|c|}
\hline & \multirow{2}{*}{\multicolumn{3}{|c|}{$\begin{array}{l}\text { Age-standardised rates in } 2013 \text { (per } \\
100000 \text { population) }\end{array}$}} & \multicolumn{6}{|c|}{ Annualised rate of change (\%) } \\
\hline & & & & \multicolumn{3}{|l|}{ 1990-2000 } & \multicolumn{3}{|l|}{ 2000-2013 } \\
\hline & Incidence & Prevalence & Mortality & Incidence & Prevalence & Mortality & Incidence & Prevalence & Mortality \\
\hline Southern Latin America & $\begin{array}{l}10 \cdot 11 \\
(7 \cdot 11 \text { to } \\
14.94)\end{array}$ & $\begin{array}{c}173 \cdot 57 \\
(144 \cdot 31 \text { to } \\
210 \cdot 86)\end{array}$ & $\begin{array}{l}3 \cdot 12(2 \cdot 23 \\
\text { to } 4 \cdot 10)\end{array}$ & $\begin{array}{l}-0 \cdot 75 \\
(-4 \cdot 84 \text { to } \\
3 \cdot 85)\end{array}$ & $\begin{array}{l}8 \cdot 44(5 \cdot 39 \\
\text { to } 12 \cdot 40)\end{array}$ & $\begin{array}{l}10 \cdot 00 \\
(6 \cdot 17 \text { to } \\
13 \cdot 76)\end{array}$ & $\begin{array}{l}-0 \cdot 24 \\
(-3 \cdot 40 \text { to } \\
3 \cdot 08)\end{array}$ & $\begin{array}{l}2 \cdot 60(1 \cdot 08 \\
\text { to } 3 \cdot 97)\end{array}$ & $\begin{array}{l}-2 \cdot 76 \\
(-5.37 \text { to } \\
-0.65)\end{array}$ \\
\hline Tropical Latin America & $\begin{array}{l}11.64 \\
(8.93 \text { to } \\
15 \cdot 83)\end{array}$ & $\begin{array}{c}188 \cdot 35 \\
(125 \cdot 90 \text { to } \\
244 \cdot 16)\end{array}$ & $\begin{array}{l}4.83(3.83 \\
\text { to } 6.09)\end{array}$ & $\begin{array}{l}-7 \cdot 15 \\
(-10 \cdot 27 \\
\text { to }-3 \cdot 64)\end{array}$ & $\begin{array}{l}2 \cdot 20(0 \cdot 13 \\
\text { to } 4 \cdot 56)\end{array}$ & $\begin{array}{l}1 \cdot 09 \\
(-2 \cdot 41 \text { to } \\
6 \cdot 19)\end{array}$ & $\begin{array}{l}0 \cdot 37 \\
(-1 \cdot 16 \text { to } \\
2 \cdot 50)\end{array}$ & $\begin{array}{l}1 \cdot 10(-0 \cdot 25 \\
\text { to } 2 \cdot 23)\end{array}$ & $\begin{array}{l}-2 \cdot 20 \\
(-4 \cdot 57 \text { to } \\
0 \cdot 01)\end{array}$ \\
\hline $\begin{array}{l}\text { North Africa and Middle } \\
\text { East }\end{array}$ & $\begin{array}{l}2 \cdot 84(1.93 \\
\text { to } 4.93)\end{array}$ & $\begin{array}{c}26 \cdot 45 \\
(21 \cdot 31 \text { to } \\
39 \cdot 26)\end{array}$ & $\begin{array}{l}1.66(1.26 \\
\text { to } 2 \cdot 39)\end{array}$ & $\begin{array}{l}11.52 \\
(0.35 \text { to } \\
25 \cdot 42)\end{array}$ & $\begin{array}{l}16 \cdot 98(7 \cdot 18 \\
\text { to } 30 \cdot 42)\end{array}$ & $\begin{array}{l}20 \cdot 66 \\
(9 \cdot 81 \text { to } \\
35 \cdot 43)\end{array}$ & $\begin{array}{l}-0 \cdot 35 \\
(-4 \cdot 01 \text { to } \\
5 \cdot 02)\end{array}$ & $\begin{array}{l}1.94(-0 \cdot 71 \\
\text { to } 5 \cdot 64)\end{array}$ & $\begin{array}{l}3.05 \\
(-0.99 \text { to } \\
7.85)\end{array}$ \\
\hline High-income North America & $\begin{array}{l}7 \cdot 43(3 \cdot 46 \\
\text { to } 17 \cdot 16)\end{array}$ & $\begin{array}{c}135.49 \\
(78 \cdot 21 \text { to } \\
220 \cdot 57)\end{array}$ & $\begin{array}{l}2.65(1.68 \\
\text { to } 3.69)\end{array}$ & $\begin{array}{l}-8 \cdot 87 \\
(-13 \cdot 56 \\
\text { to }-3 \cdot 10)\end{array}$ & $\begin{array}{l}-3 \cdot 31 \\
(-6 \cdot 16 \text { to } \\
-1 \cdot 52)\end{array}$ & $\begin{array}{l}-10 \cdot 20 \\
(-13.49 \\
\text { to }-7.99)\end{array}$ & $\begin{array}{l}2 \cdot 01 \\
(-3 \cdot 23 \text { to } \\
9 \cdot 49)\end{array}$ & $\begin{array}{l}1.24(-1.09 \\
\text { to } 4.97)\end{array}$ & $\begin{array}{l}-2.89 \\
(-5.95 \text { to } \\
-0.10)\end{array}$ \\
\hline Oceania & $\begin{array}{l}16 \cdot 09 \\
(11.07 \text { to } \\
22 \cdot 43)\end{array}$ & $\begin{array}{c}341.52 \\
(287.06 \text { to } \\
403.52)\end{array}$ & $\begin{array}{c}18 \cdot 80 \\
(13 \cdot 86 \text { to } \\
25 \cdot 16)\end{array}$ & $\begin{array}{l}15 \cdot 64 \\
(5 \cdot 25 \text { to } \\
27 \cdot 08)\end{array}$ & $\begin{array}{l}23 \cdot 43 \\
(17 \cdot 21 \text { to } \\
30 \cdot 47)\end{array}$ & $\begin{array}{l}31.68 \\
(27.45 \text { to } \\
36.49)\end{array}$ & $\begin{array}{l}-9.47 \\
(-11.74 \\
\text { to }-7.34)\end{array}$ & $\begin{array}{l}0 \cdot 30(-2 \cdot 04 \\
\text { to } 3 \cdot 13)\end{array}$ & $\begin{array}{l}1 \cdot 21 \\
(-3 \cdot 80 \text { to } \\
6 \cdot 92)\end{array}$ \\
\hline Central sub-Saharan Africa & $\begin{array}{l}94.53 \\
\text { (78.72 to } \\
113.99)\end{array}$ & $\begin{array}{c}1328 \cdot 37 \\
(1222 \cdot 40 \text { to } \\
1439 \cdot 78)\end{array}$ & $\begin{array}{c}94.45 \\
(81 \cdot 17 \text { to } \\
111 \cdot 04)\end{array}$ & $\begin{array}{l}-0.25 \\
(-1.66 \text { to } \\
0.93)\end{array}$ & $\begin{array}{l}2.92(1.96 \\
\text { to } 3.97)\end{array}$ & $\begin{array}{l}5.71(3.57 \\
\text { to } 8 \cdot 17)\end{array}$ & $\begin{array}{l}-3 \cdot 89 \\
(-5 \cdot 15 \text { to } \\
-2 \cdot 60)\end{array}$ & $\begin{array}{l}-1.43 \\
(-2.09 \text { to } \\
-0.78)\end{array}$ & $\begin{array}{l}-2.42 \\
(-3.47 \text { to } \\
-1 \cdot 14)\end{array}$ \\
\hline Eastern sub-Saharan Africa & $\begin{array}{l}177.08 \\
(163.51 \text { to } \\
191.29)\end{array}$ & $\begin{array}{c}3416 \cdot 89 \\
(3249 \cdot 05 \text { to } \\
3609 \cdot 25)\end{array}$ & $\begin{array}{c}164 \cdot 58 \\
(154 \cdot 75 \\
\text { to } \\
175 \cdot 06)\end{array}$ & $\begin{array}{l}-3 \cdot 87 \\
(-4 \cdot 99 \text { to } \\
-2 \cdot 81)\end{array}$ & $\begin{array}{l}5 \cdot 92(5 \cdot 27 \\
\text { to } 6 \cdot 51)\end{array}$ & $\begin{array}{l}14 \cdot 50 \\
(12 \cdot 89 \text { to } \\
16 \cdot 00)\end{array}$ & $\begin{array}{l}-4 \cdot 40 \\
(-5 \cdot 14 \text { to } \\
-3 \cdot 65)\end{array}$ & $\begin{array}{l}-1.74 \\
(-1.98 \text { to } \\
-1.51)\end{array}$ & $\begin{array}{l}-3 \cdot 78 \\
(-4.82 \text { to } \\
-2 \cdot 65)\end{array}$ \\
\hline Southern sub-Saharan Africa & $\begin{array}{l}647 \cdot 13 \\
(606 \cdot 40 \text { to } \\
693 \cdot 70)\end{array}$ & $\begin{array}{c}11850 \cdot 47 \\
(11368 \cdot 38 \\
\text { to } 12 \\
327 \cdot 29)\end{array}$ & $\begin{array}{c}511.41 \\
(467.65 \\
\text { to } \\
556 \cdot 63)\end{array}$ & $\begin{array}{l}10 \cdot 60 \\
(9 \cdot 80 \text { to } \\
11 \cdot 47)\end{array}$ & $\begin{array}{l}21 \cdot 69 \\
(21 \cdot 08 \text { to } \\
22 \cdot 58)\end{array}$ & $\begin{array}{l}31 \cdot 17 \\
(28 \cdot 62 \text { to } \\
33 \cdot 63)\end{array}$ & $\begin{array}{l}-4 \cdot 58 \\
(-5 \cdot 16 \text { to } \\
-4 \cdot 00)\end{array}$ & $\begin{array}{l}1 \cdot 13(0 \cdot 90 \\
\text { to } 1 \cdot 35)\end{array}$ & $\begin{array}{l}3.41(1.54 \\
\text { to } 4 \cdot 88)\end{array}$ \\
\hline Western sub-Saharan Africa & $\begin{array}{l}90 \cdot 89 \\
(77 \cdot 20 \text { to } \\
107 \cdot 09)\end{array}$ & $\begin{array}{c}1833.57 \\
(1692.97 \text { to } \\
1999 \cdot 25)\end{array}$ & $\begin{array}{c}114 \cdot 36 \\
(103 \cdot 20 \\
\text { to } \\
127 \cdot 44)\end{array}$ & $\begin{array}{l}5 \cdot 79(3.88 \\
\text { to } 8 \cdot 08)\end{array}$ & $\begin{array}{l}13 \cdot 12 \\
(11.69 \text { to } \\
14 \cdot 69)\end{array}$ & $\begin{array}{l}18 \cdot 75 \\
(16 \cdot 65 \text { to } \\
21 \cdot 01)\end{array}$ & $\begin{array}{l}-7 \cdot 26 \\
(-8 \cdot 40 \text { to } \\
-6 \cdot 19)\end{array}$ & $\begin{array}{l}-0.84 \\
(-1.56 \text { to } \\
-0.20)\end{array}$ & $\begin{array}{l}0 \cdot 82 \\
(-0 \cdot 38 \text { to } \\
2 \cdot 01)\end{array}$ \\
\hline
\end{tabular}

Data in parentheses are 95\% uncertainty intervals. 


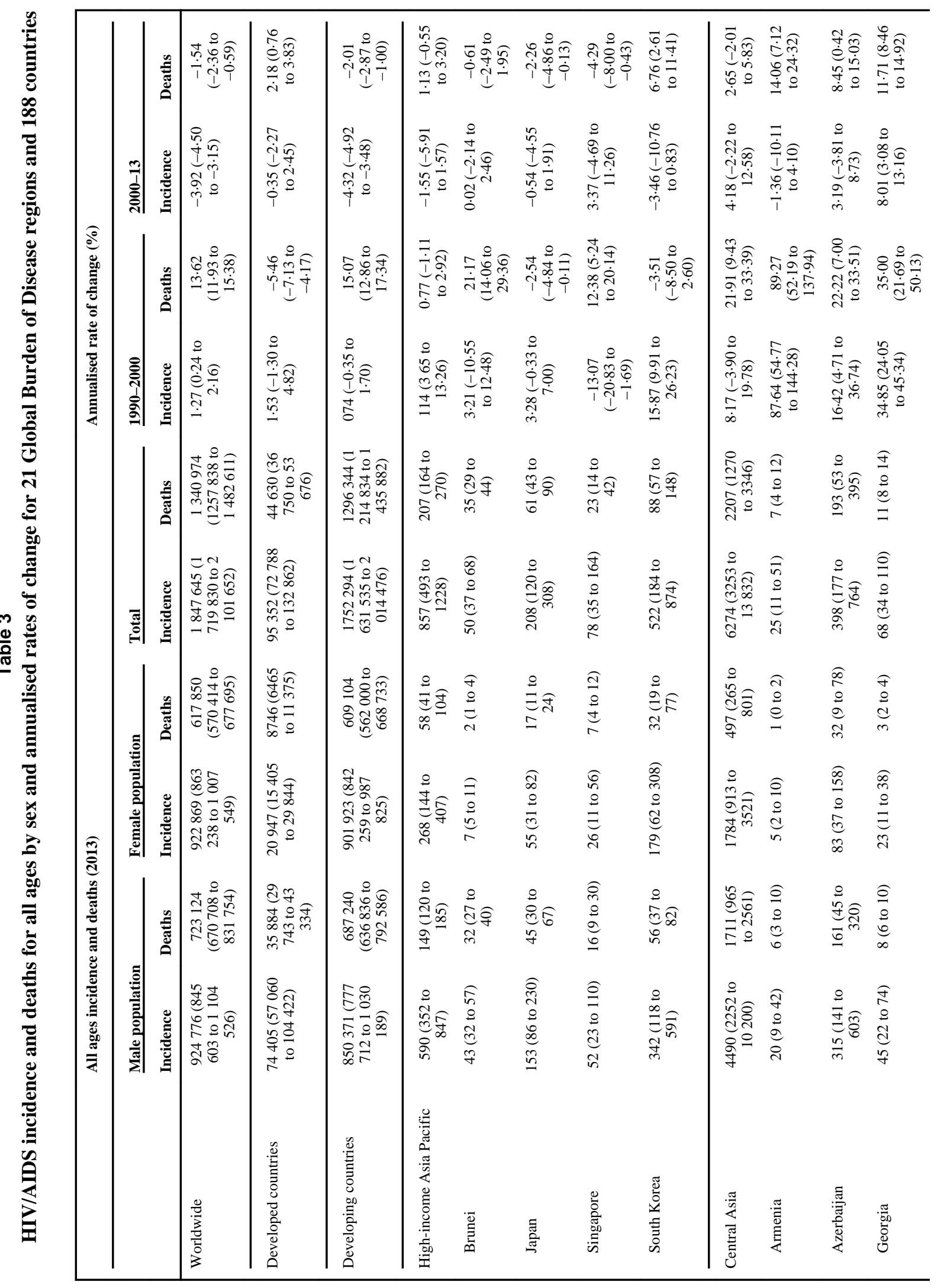




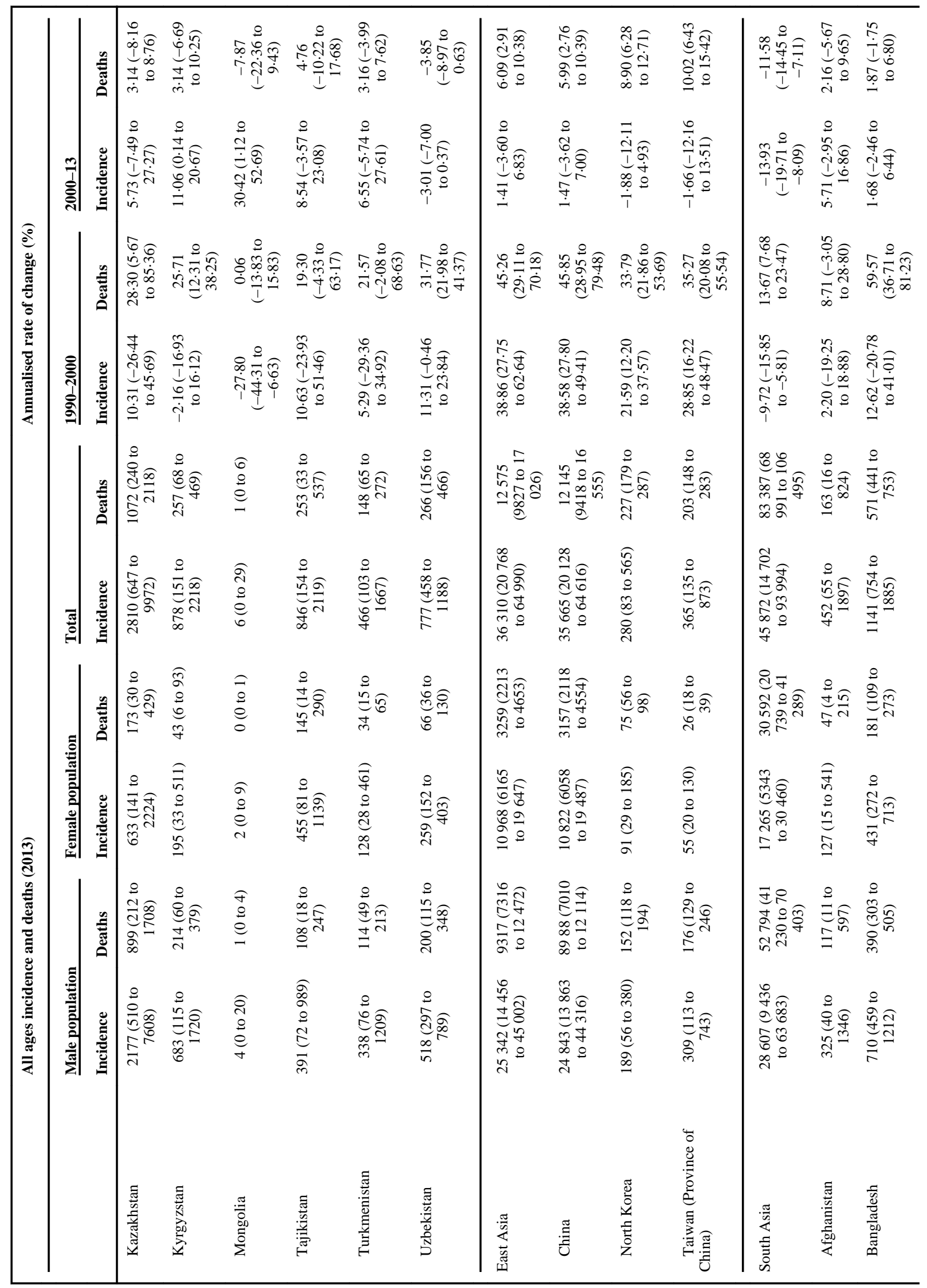




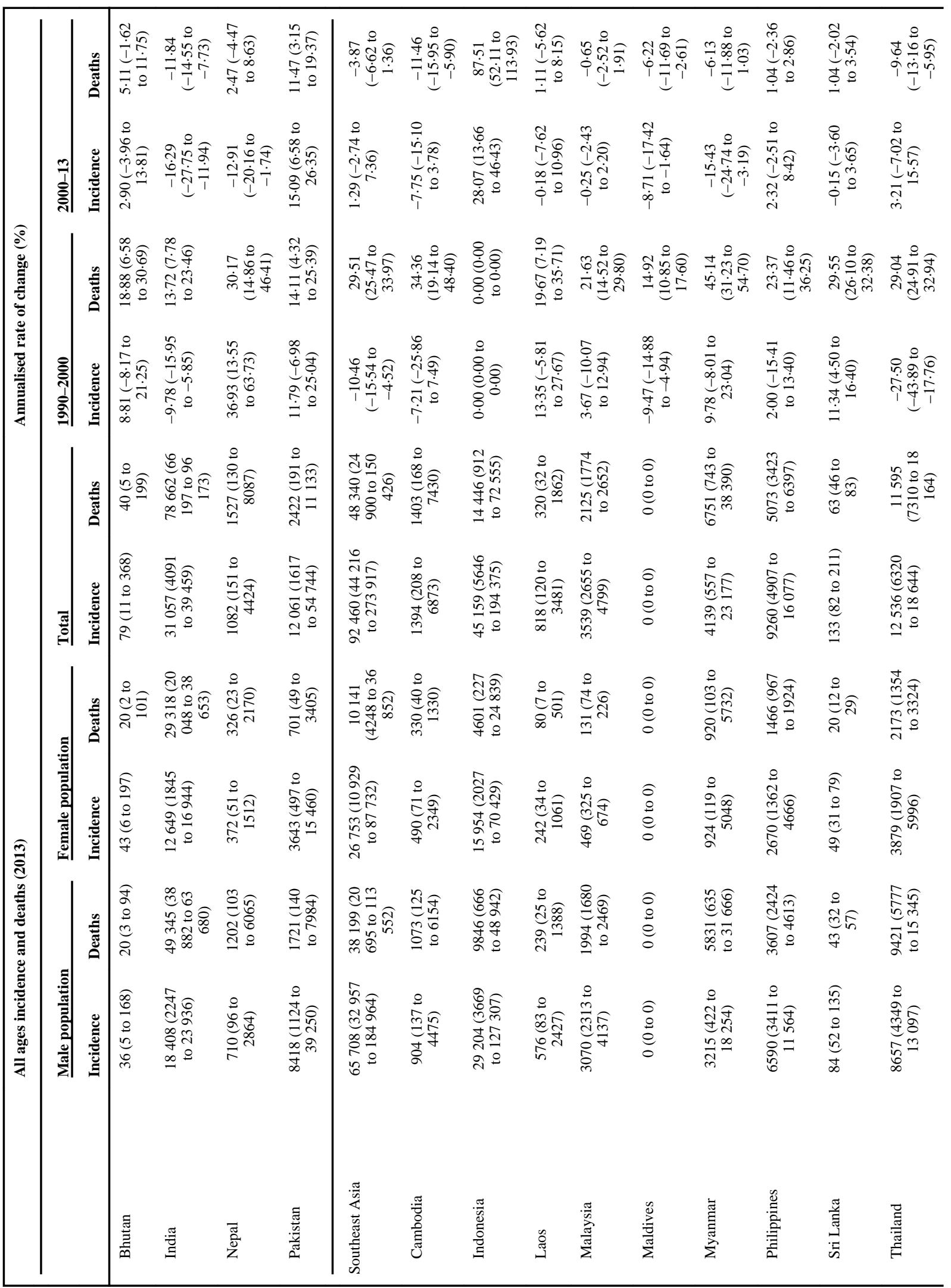




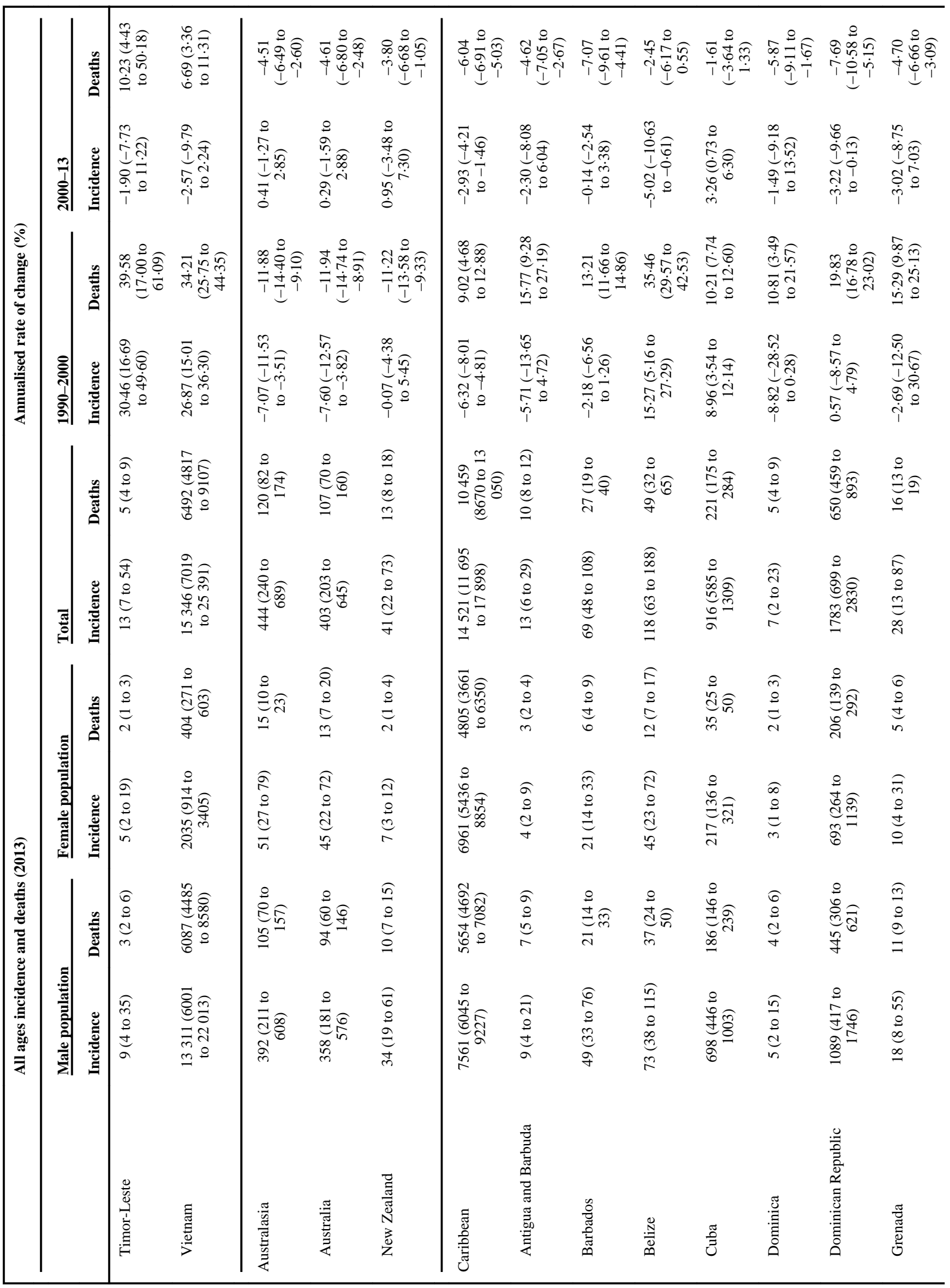




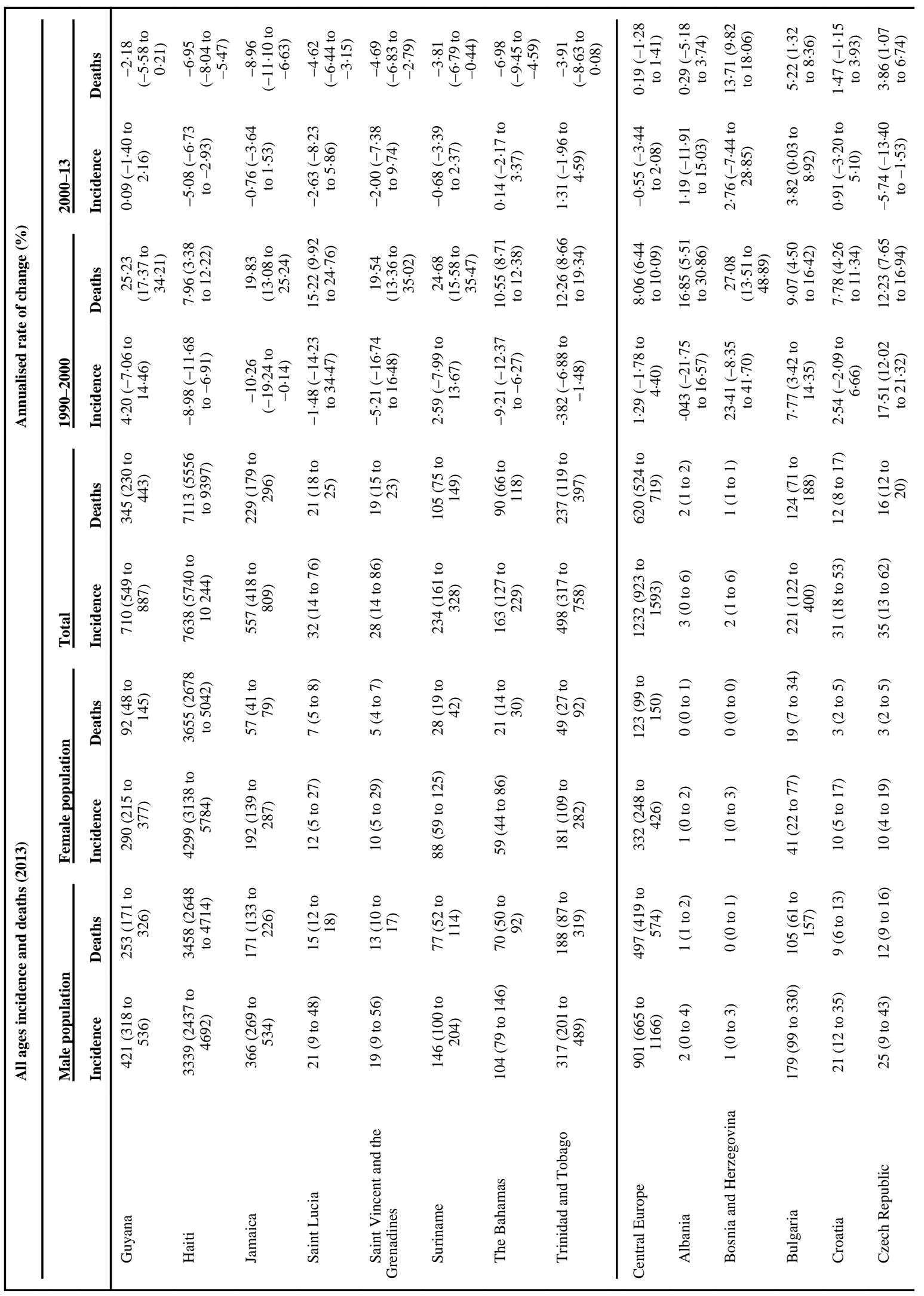




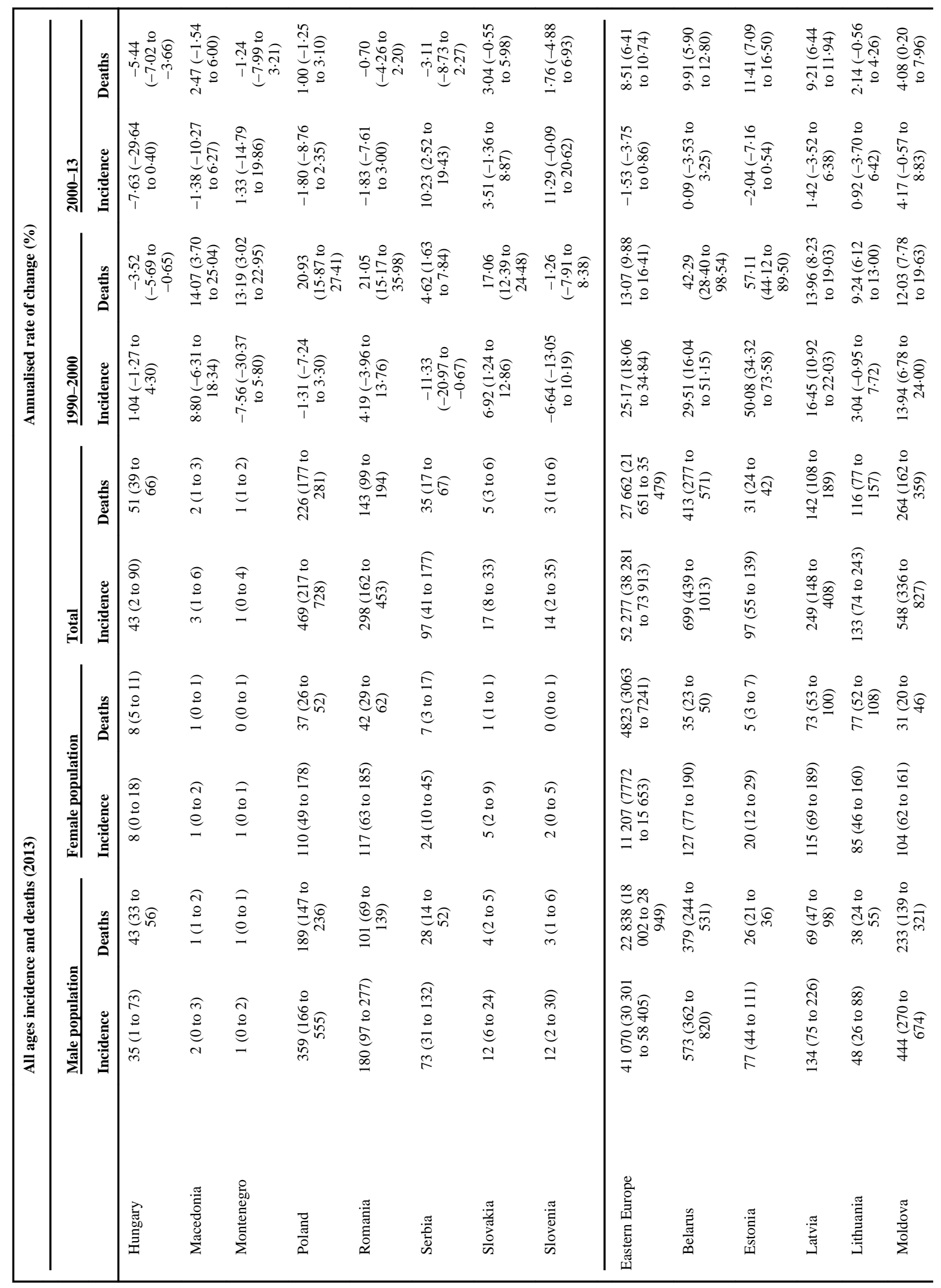




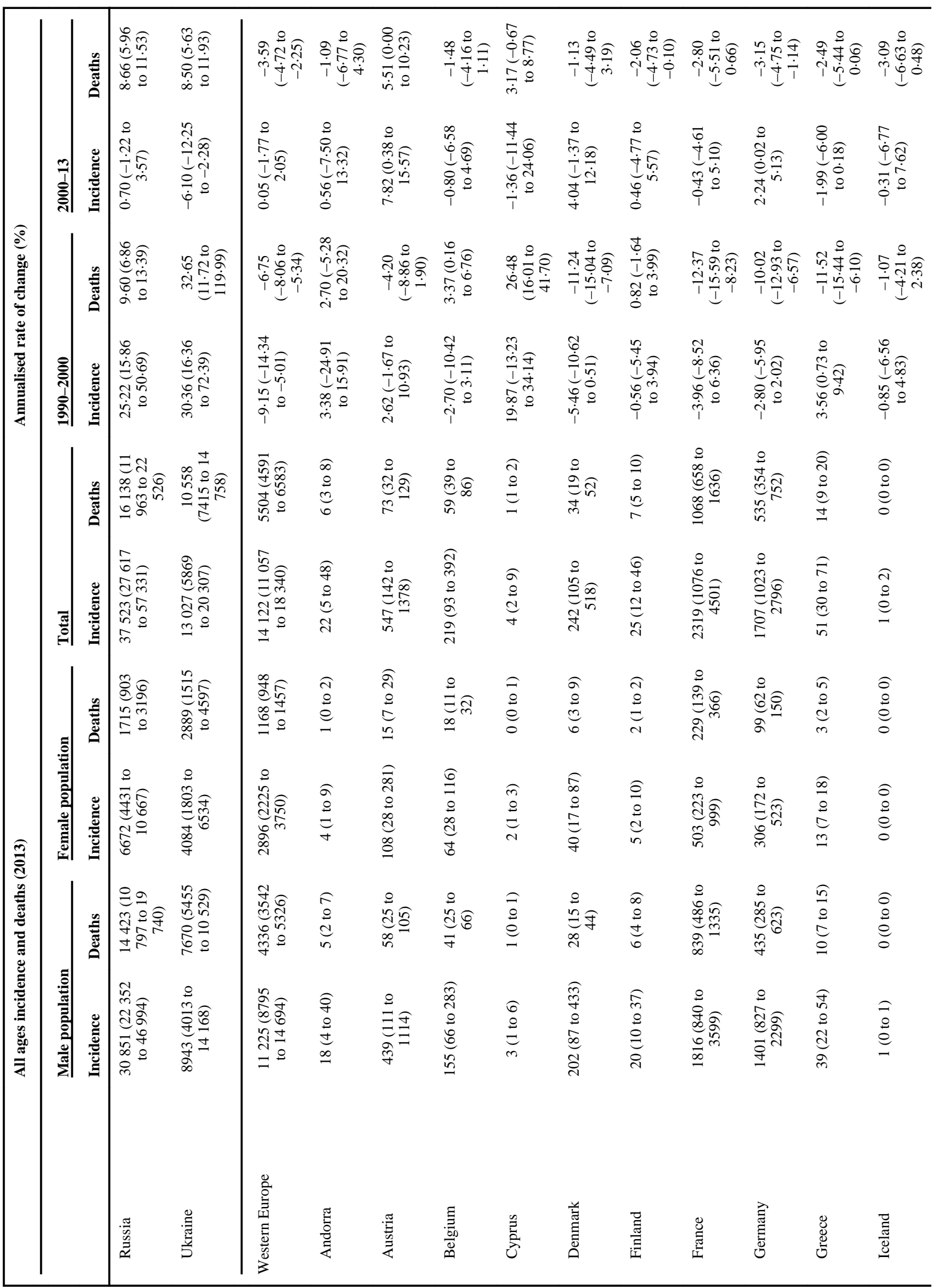




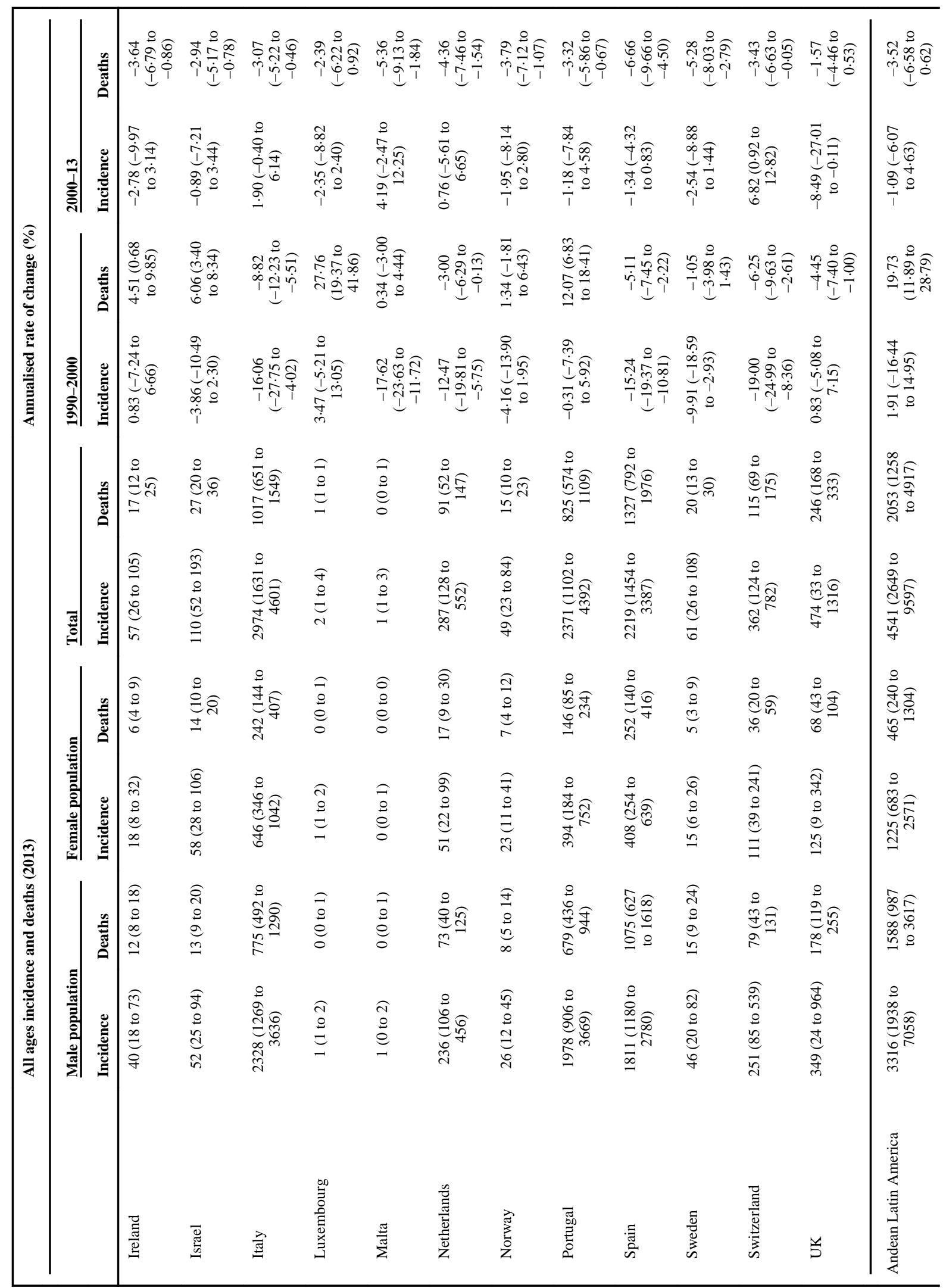




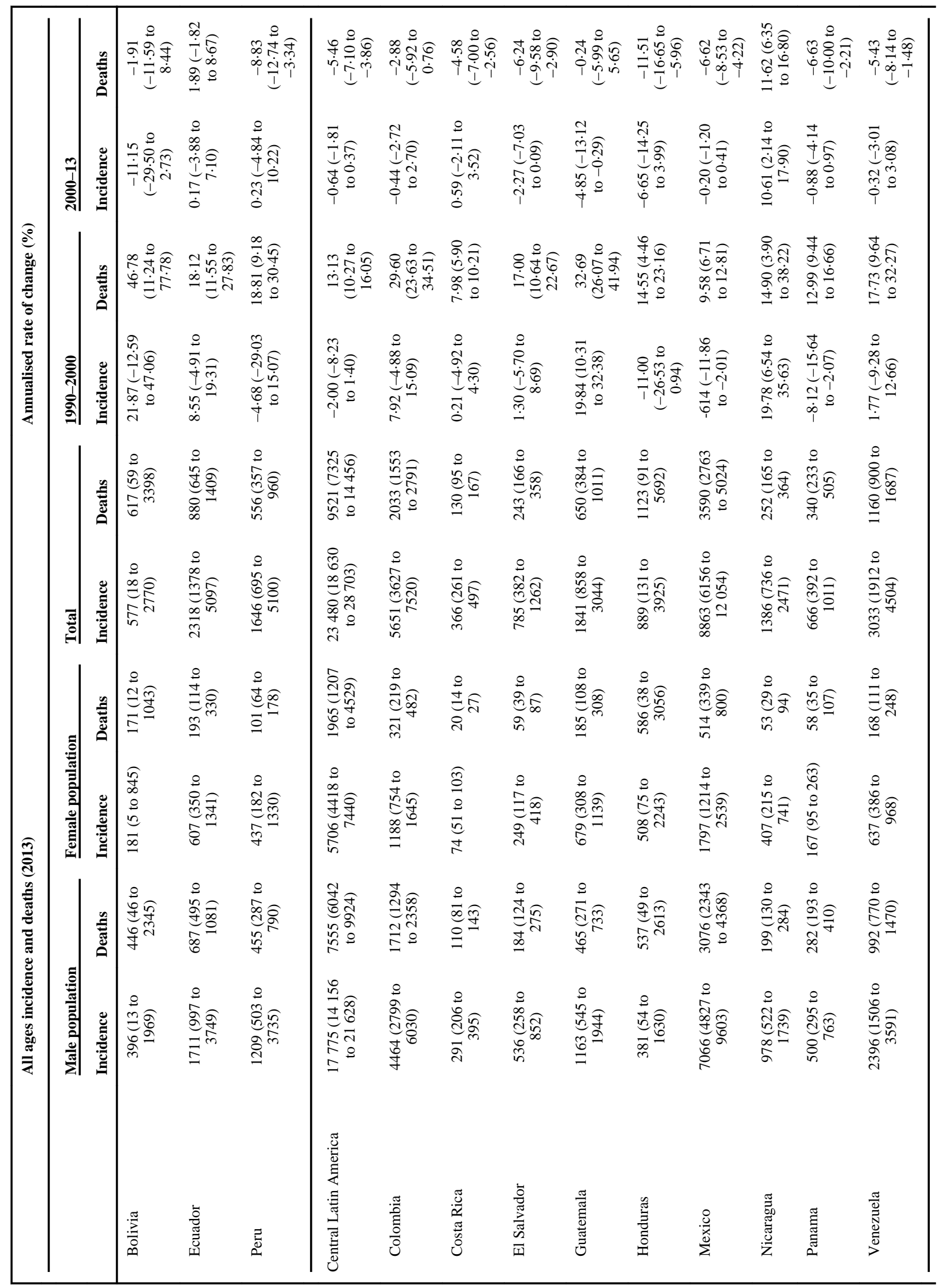




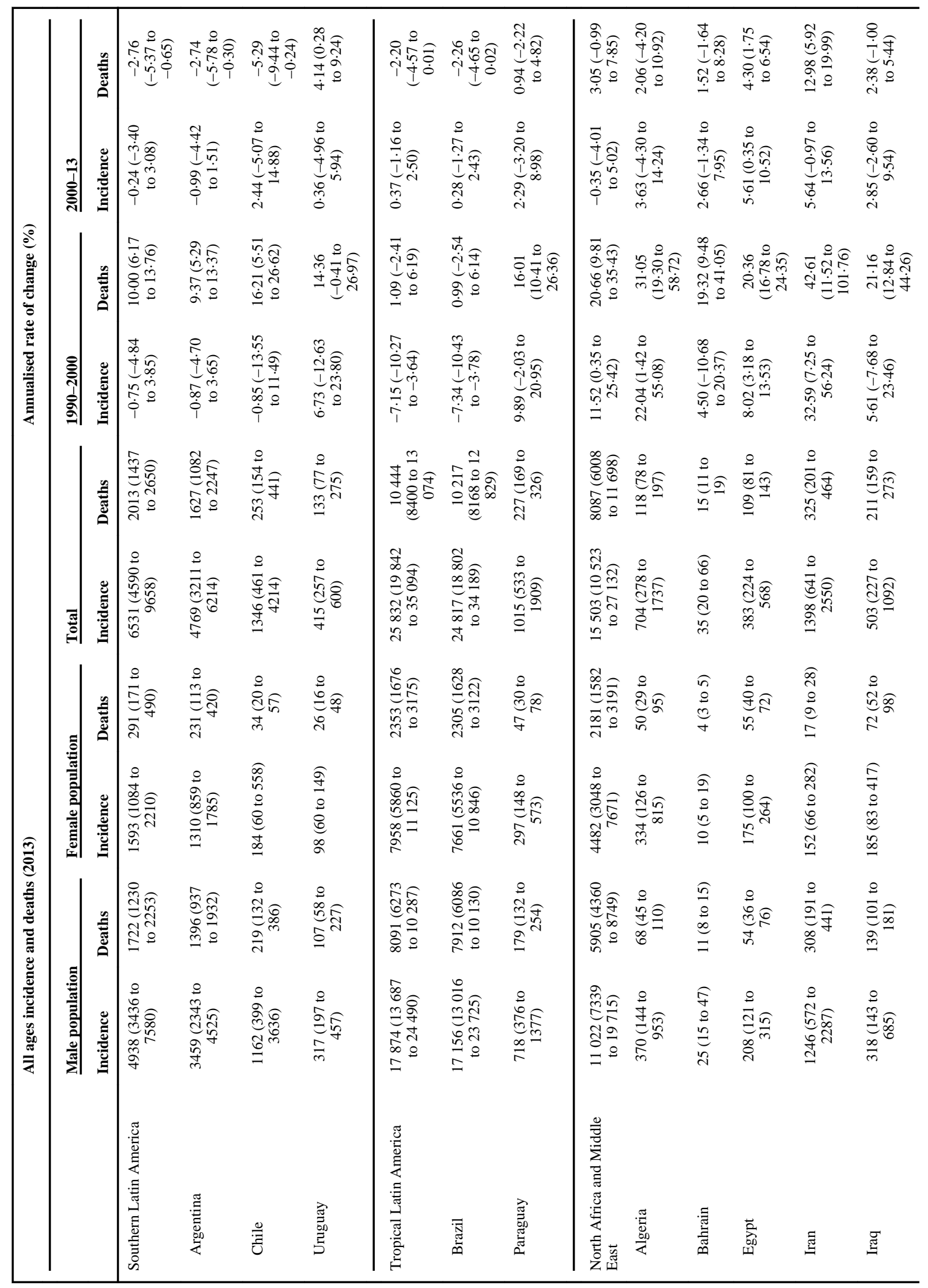




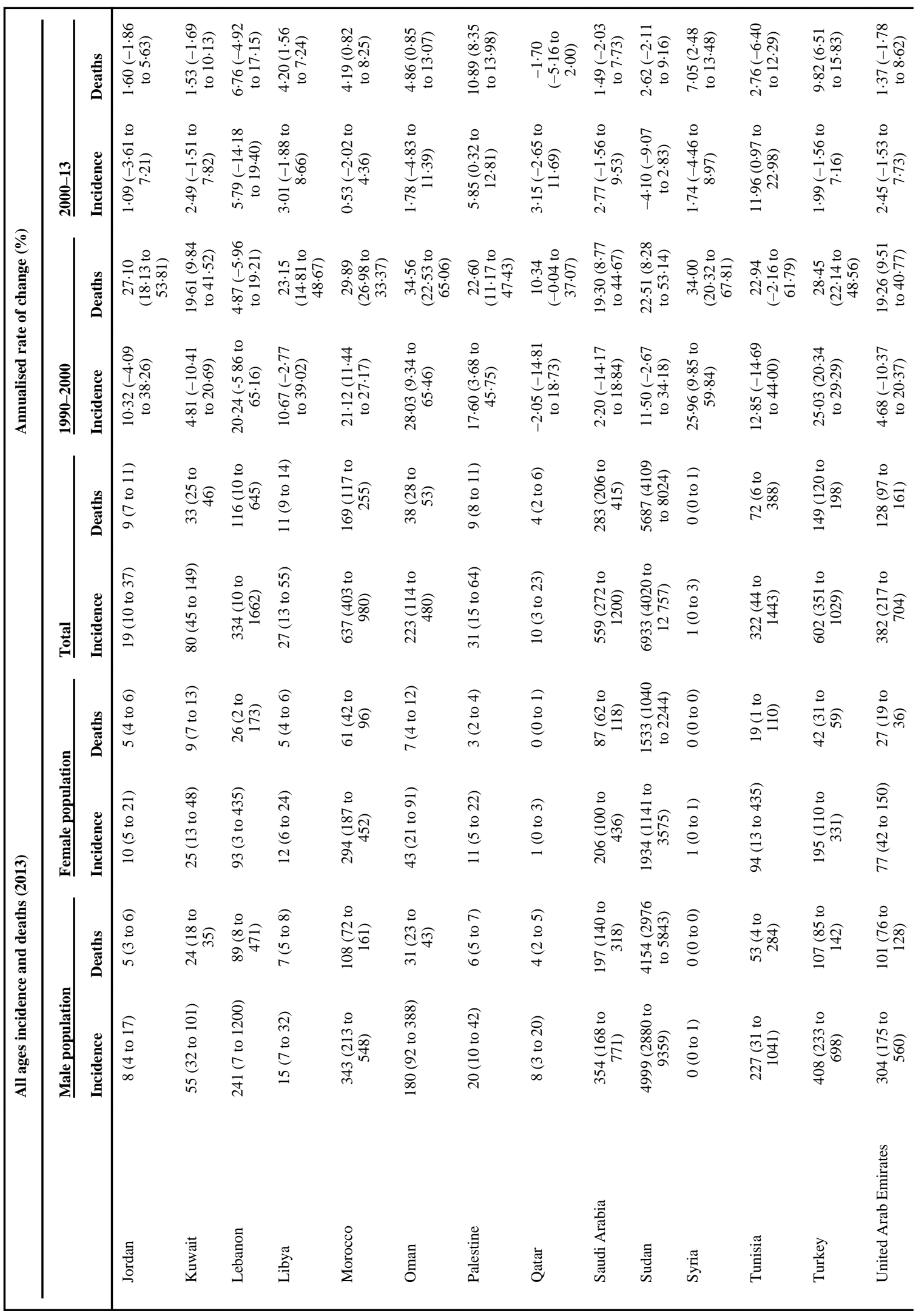




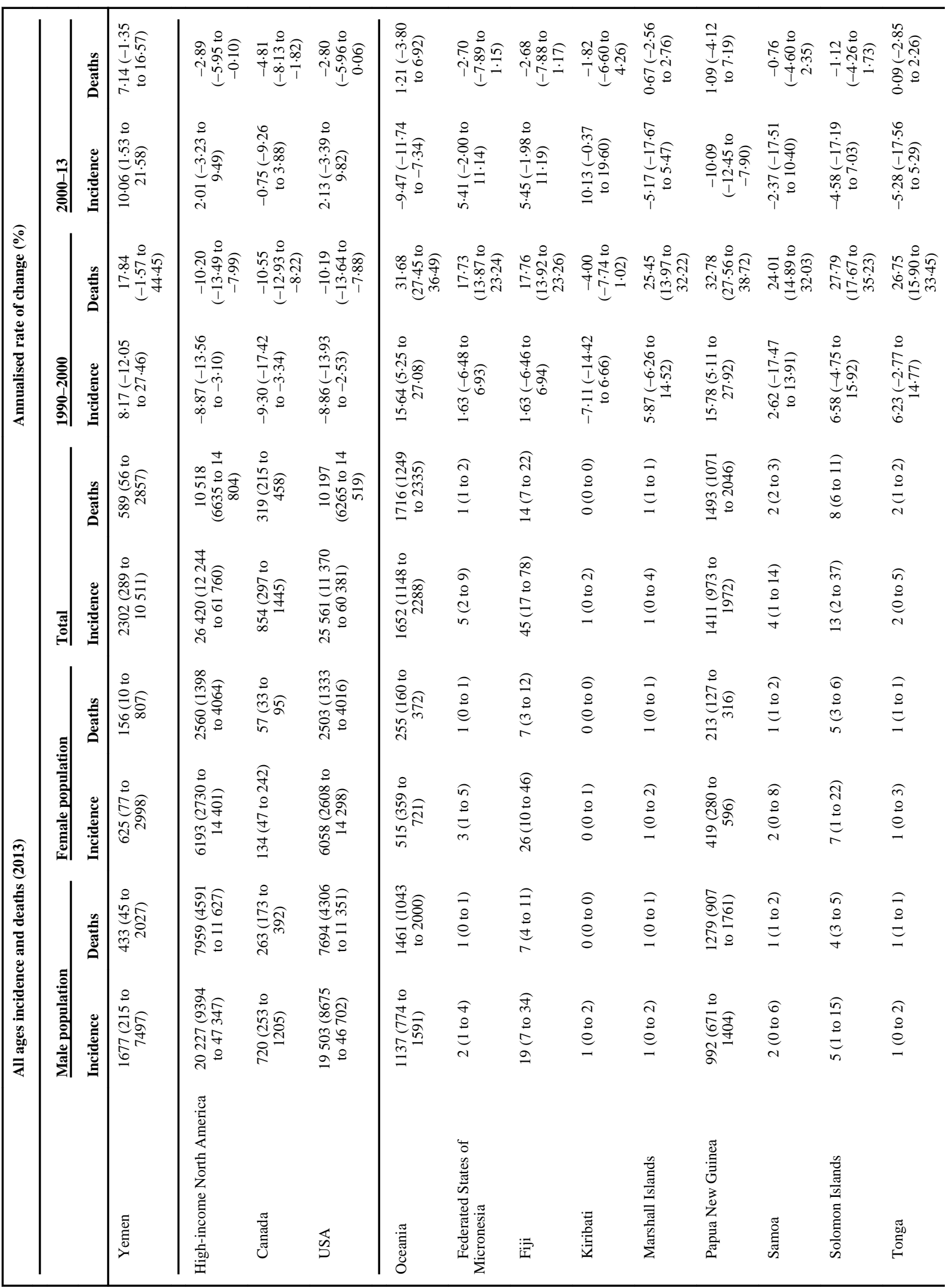




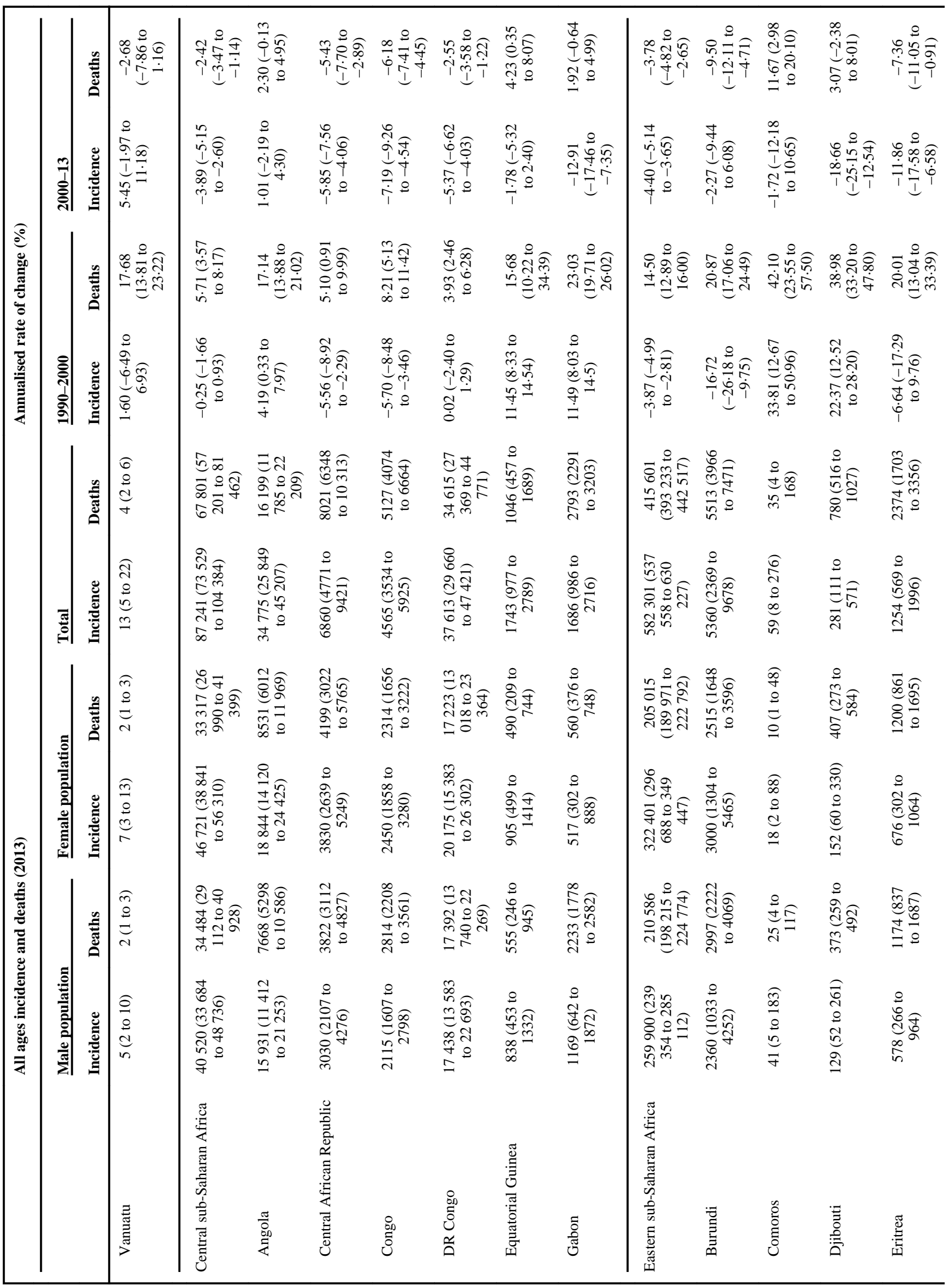




\begin{tabular}{|c|c|c|c|c|c|c|c|c|c|c|c|c|c|c|c|}
\hline & & 䔍 & 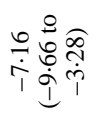 & 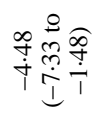 & 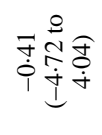 & 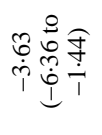 & 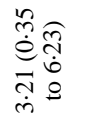 & 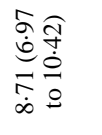 & 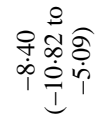 & 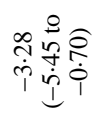 & $\begin{array}{l}\frac{n}{\dot{m}} \hat{\rho} \\
\dot{i} \\
0 \\
\dot{\sim}\end{array}$ & 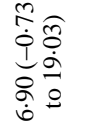 & 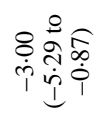 & 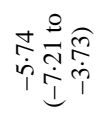 & 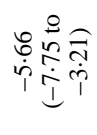 \\
\hline & 章| & 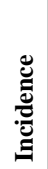 & 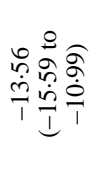 & 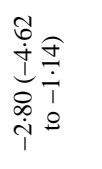 & 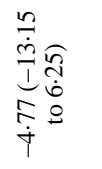 & 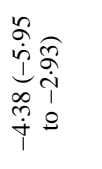 & 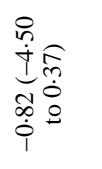 & 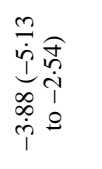 & 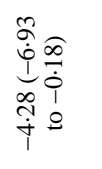 & 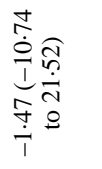 & 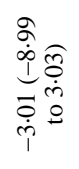 & 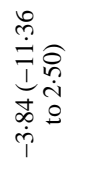 & 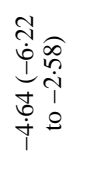 & 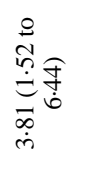 & 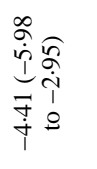 \\
\hline 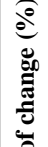 & 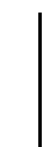 & 㽕 & 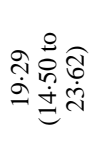 & 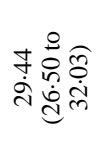 & 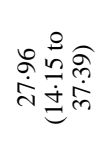 & 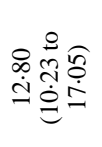 & 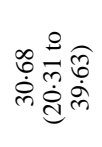 & 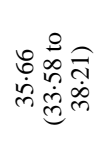 & 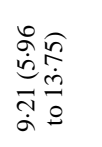 & 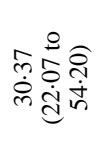 & 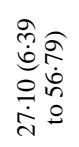 & $\begin{array}{l}\infty \\
\infty \\
\stackrel{\infty}{\infty} \\
0 \\
0 \\
\dot{\infty} \\
\dot{\infty} \\
\stackrel{\infty}{\infty}\end{array}$ & 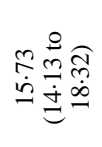 & 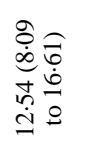 & 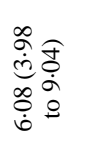 \\
\hline 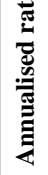 & 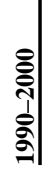 & 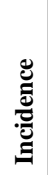 & 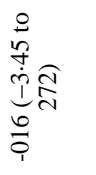 & 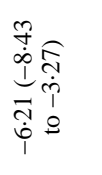 & 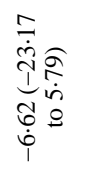 & 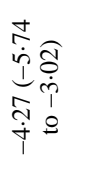 & 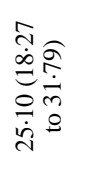 & 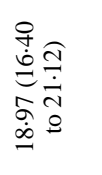 & 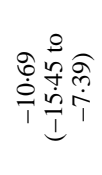 & 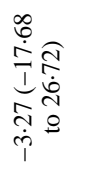 & 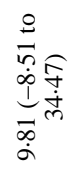 & 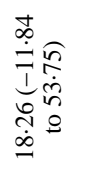 & 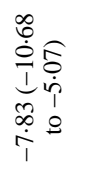 & 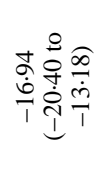 & 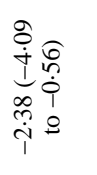 \\
\hline & & $\stackrel{\mathscr{a}}{\bar{E}}$ & 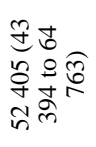 & 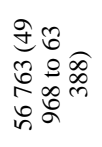 & 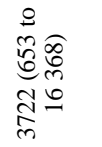 & 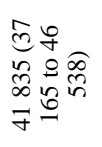 & 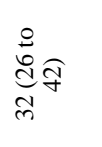 & 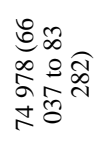 & 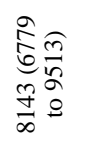 & $\begin{array}{l}\stackrel{f}{d} \\
\stackrel{9}{d} \\
\stackrel{m}{m}\end{array}$ & 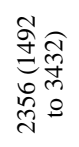 & 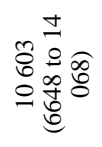 & 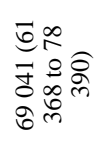 & 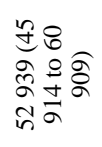 & 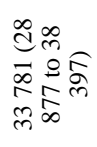 \\
\hline & है। & 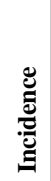 & 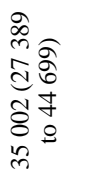 & 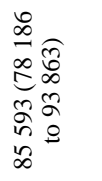 & 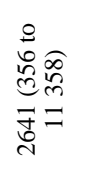 & 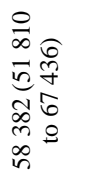 & 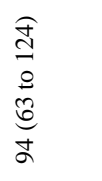 & 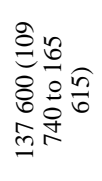 & 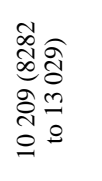 & $\begin{array}{l}0 \\
0 \\
\stackrel{2}{1} \\
= \\
n\end{array}$ & 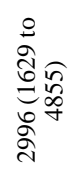 & 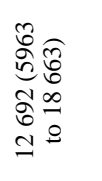 & 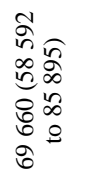 & 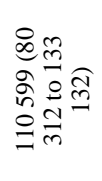 & 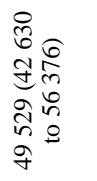 \\
\hline & & 苛 & 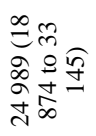 & 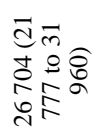 & 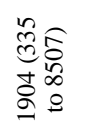 & 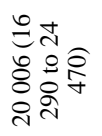 & $\begin{array}{l}0 \\
0 \\
0 \\
0 \\
n\end{array}$ & 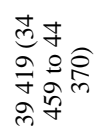 & 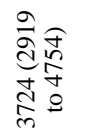 & 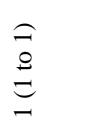 & 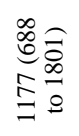 & 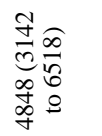 & 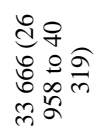 & 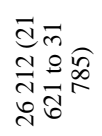 & 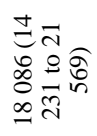 \\
\hline$\widehat{m}$ & 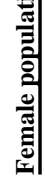 & 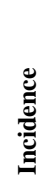 & 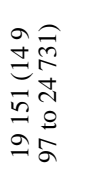 & 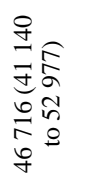 & $\begin{array}{l}\stackrel{0}{\infty} \\
\underset{\infty}{\infty} \widehat{\infty} \\
\stackrel{\infty}{\infty} \\
\infty \\
\infty\end{array}$ & 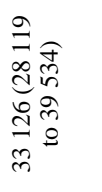 & $\begin{array}{l}0 \\
0 \\
0 \\
0 \\
\text { I } \\
0 \\
2\end{array}$ & 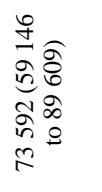 & 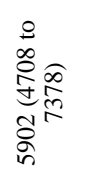 & $\begin{array}{l}6 \\
0 \\
0 \\
0 \\
i\end{array}$ & 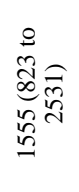 & 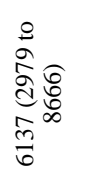 & 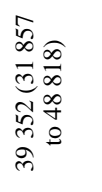 & 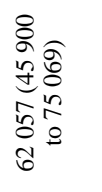 & 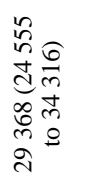 \\
\hline 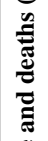 & & 营 & 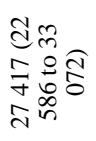 & 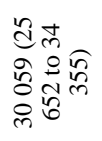 & 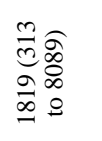 & 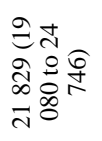 & 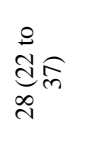 & 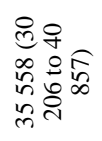 & 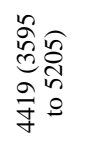 & 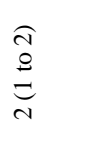 & 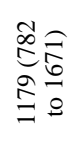 & 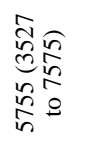 & 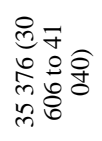 & 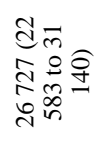 & 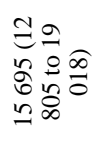 \\
\hline 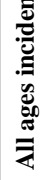 & 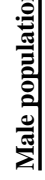 & 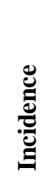 & 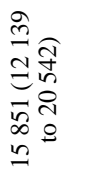 & 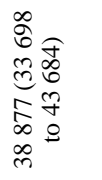 & 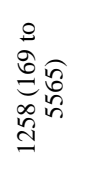 & 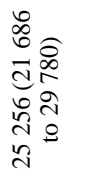 & 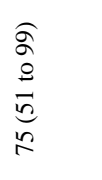 & 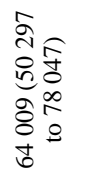 & 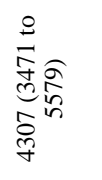 & $\begin{array}{l}\widehat{\widehat{A}} \\
\stackrel{0}{0} \\
e \\
e\end{array}$ & 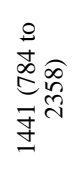 & 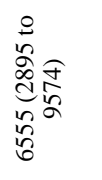 & 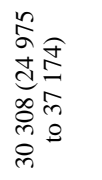 & 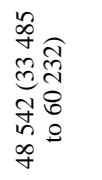 & 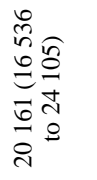 \\
\hline & & & 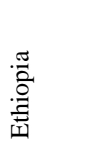 & 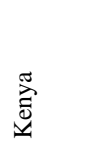 & 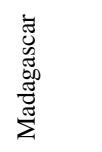 & $\frac{\sqrt[5]{5}}{\sum}$ & 莺 & 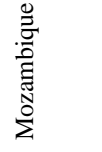 & 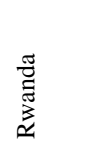 & $\begin{array}{l}\frac{0}{0} \\
\overline{0} \\
\frac{0}{0} \\
\frac{0}{0} \\
\tilde{n}\end{array}$ & 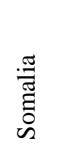 & 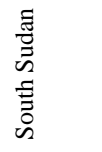 & 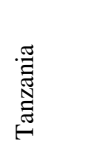 & 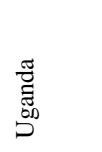 & 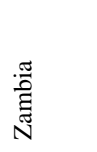 \\
\hline
\end{tabular}




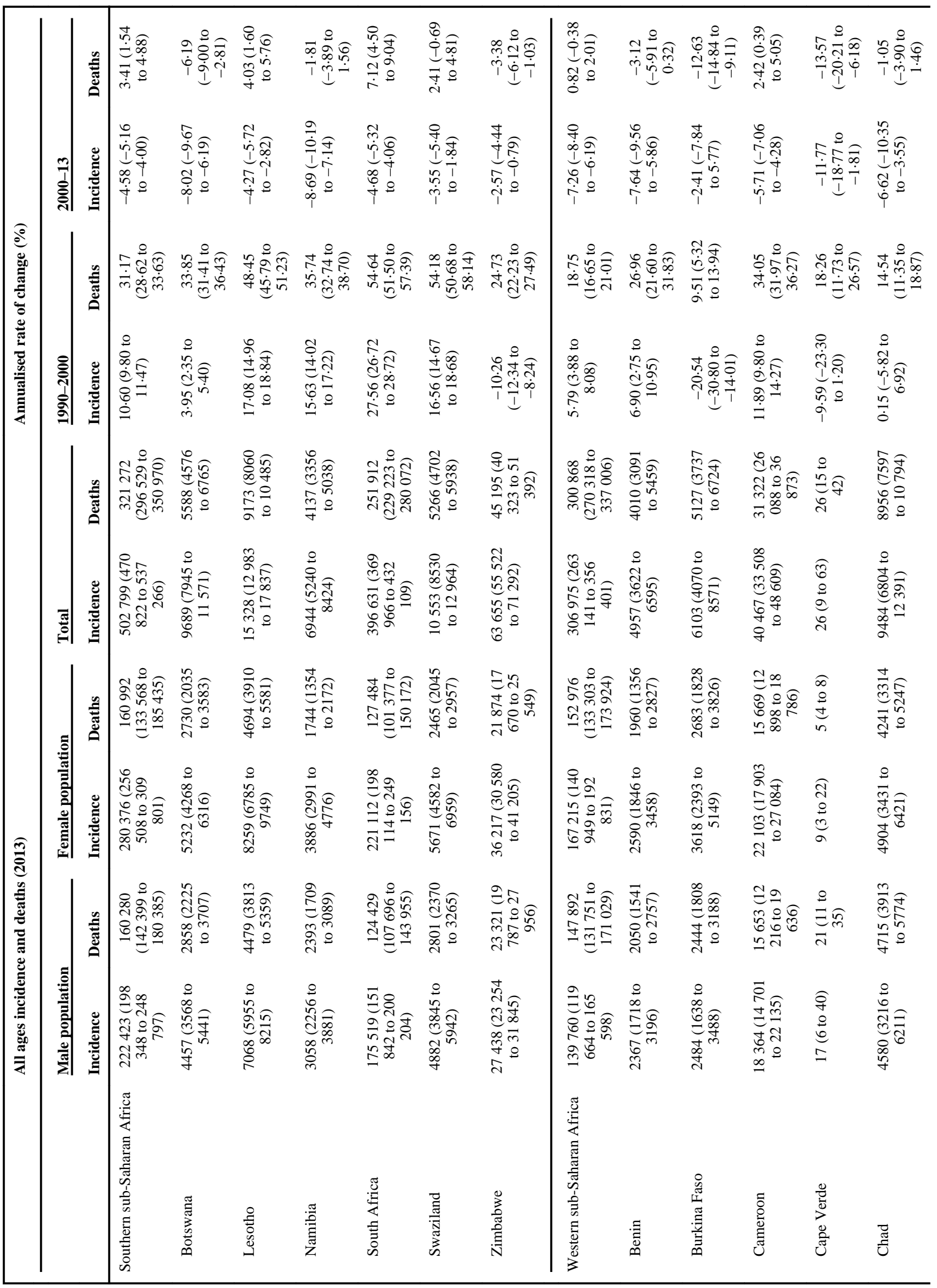




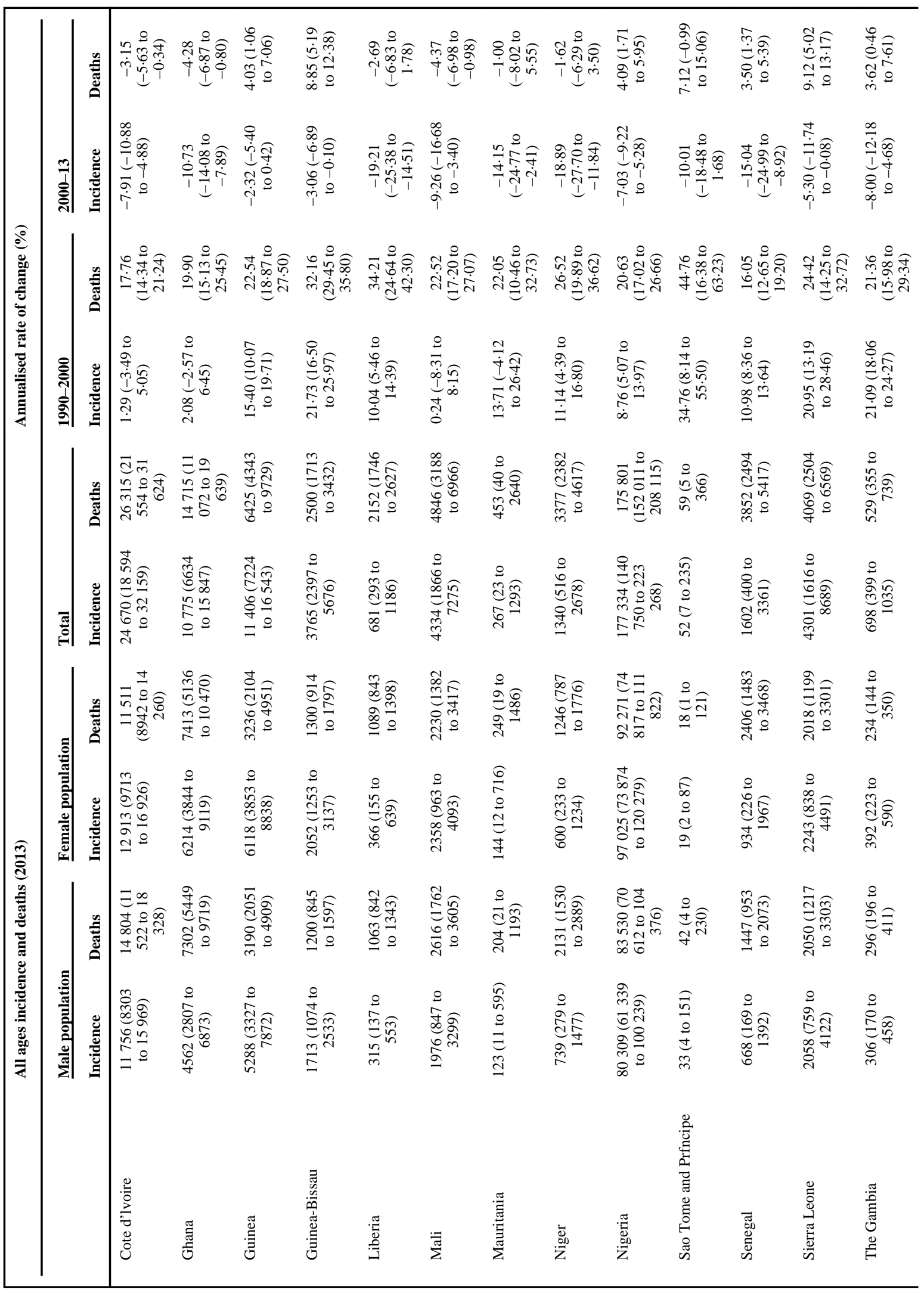




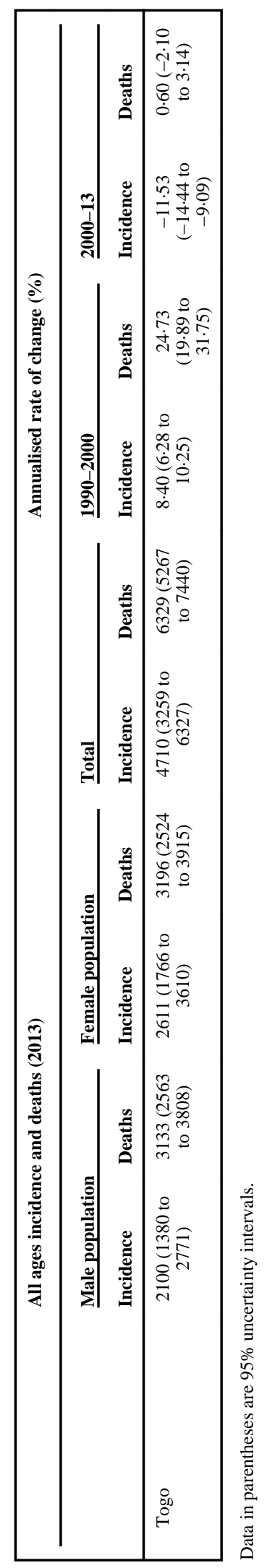


Table 4

Age-standardised tuberculosis without HIV incidence, prevalence, and mortality rates and annualised rates of change for both sexes for 21 Global Burden of Disease regions

\begin{tabular}{|c|c|c|c|c|c|c|c|c|c|}
\hline & \multirow{2}{*}{\multicolumn{3}{|c|}{$\begin{array}{l}\text { Age-standardisedrates in } 2013 \text { (per } \\
100000 \text { population) }\end{array}$}} & \multicolumn{6}{|c|}{ Annualised rate of change (\%) } \\
\hline & & & & \multicolumn{3}{|l|}{ 1990-2000 } & \multicolumn{3}{|l|}{ 2000-2013 } \\
\hline & Incidence & Prevalence & Mortality & Incidence & Prevalence & Mortality & Incidence & Prevalence & Mortality \\
\hline Worldwide & $\begin{array}{l}98 \cdot 65 \\
(96 \cdot 16 \text { to } \\
101 \cdot 43)\end{array}$ & $\begin{array}{l}159 \cdot 00 \\
(154 \cdot 14 \text { to } \\
164 \cdot 10)\end{array}$ & $\begin{array}{l}19 \cdot 24 \\
(17 \cdot 44 \text { to } \\
20 \cdot 98)\end{array}$ & $\begin{array}{l}0.03 \\
(-0 \cdot 17 \text { to } \\
0 \cdot 25)\end{array}$ & $\begin{array}{l}0 \cdot 41(0 \cdot 23 \\
\text { to } 0 \cdot 58)\end{array}$ & $-3 \cdot 29(-4 \cdot 07$ to $-2 \cdot 58)$ & $\begin{array}{l}-0.60 \\
(-0.73 \text { to } \\
-0.50)\end{array}$ & $\begin{array}{l}-1 \cdot 31 \\
(-1.41 \text { to } \\
-1 \cdot 20)\end{array}$ & $\begin{array}{l}-3 \cdot 72 \\
(-4.42 \text { to } \\
-2.99)\end{array}$ \\
\hline High-income Asia Pacific & $\begin{array}{l}34 \cdot 36 \\
(33 \cdot 42 \text { to } \\
35 \cdot 26)\end{array}$ & $\begin{array}{l}62 \cdot 80 \\
(59 \cdot 34 \text { to } \\
66 \cdot 56)\end{array}$ & $\begin{array}{l}2 \cdot 14(1 \cdot 91 \\
\text { to } 2 \cdot 59)\end{array}$ & $\begin{array}{l}-0 \cdot 04 \\
(-0 \cdot 27 \text { to } \\
0 \cdot 20)\end{array}$ & $\begin{array}{l}-1.64 \\
(-1.99 \text { to } \\
-1.30)\end{array}$ & $\begin{array}{l}-6 \cdot 22(-6 \cdot 85 \text { to } \\
-5 \cdot 42)\end{array}$ & $\begin{array}{l}0 \cdot 11 \\
(-0 \cdot 06 \text { to } \\
0 \cdot 27)\end{array}$ & $\begin{array}{l}0 \cdot 13(-0 \cdot 18 \\
\text { to } 0 \cdot 40)\end{array}$ & $\begin{array}{l}-5 \cdot 03 \\
(-5 \cdot 85 \text { to } \\
-3 \cdot 99)\end{array}$ \\
\hline Central Asia & $\begin{array}{l}122 \cdot 11 \\
(118 \cdot 41 \text { to } \\
125 \cdot 73)\end{array}$ & $\begin{array}{l}179.30 \\
(172 \cdot 60 \text { to } \\
186 \cdot 59)\end{array}$ & $\begin{array}{l}11.08 \\
(7.89 \text { to } \\
12.68)\end{array}$ & $\begin{array}{l}1 \cdot 03(0 \cdot 77 \\
\text { to } 1 \cdot 32)\end{array}$ & $\begin{array}{l}1 \cdot 03(0 \cdot 82 \\
\text { to } 1 \cdot 26)\end{array}$ & $5 \cdot 50(0.65$ to $6 \cdot 52)$ & $\begin{array}{l}-0.76 \\
(-0.92 \text { to } \\
-0.58)\end{array}$ & $\begin{array}{l}-0.68 \\
(-0.85 \text { to } \\
-0.50)\end{array}$ & $\begin{array}{l}-4.97 \\
(-5.83 \text { to } \\
-4.07)\end{array}$ \\
\hline East Asia & $\begin{array}{l}74 \cdot 16 \\
(71.90 \text { to } \\
76 \cdot 54)\end{array}$ & $\begin{array}{l}130 \cdot 70 \\
(125 \cdot 21 \text { to } \\
136 \cdot 66)\end{array}$ & $\begin{array}{l}3.44(3.00 \\
\text { to } 3.99)\end{array}$ & $\begin{array}{l}0 \cdot 23 \\
(-0 \cdot 09 \text { to } \\
0 \cdot 60)\end{array}$ & $\begin{array}{l}0 \cdot 68(0 \cdot 34 \\
\text { to } 1 \cdot 00)\end{array}$ & $\begin{array}{l}-6 \cdot 70(-7 \cdot 93 \text { to } \\
-5 \cdot 73)\end{array}$ & $\begin{array}{l}-2 \cdot 08 \\
(-2 \cdot 36 \text { to } \\
-1 \cdot 85)\end{array}$ & $\begin{array}{l}-3 \cdot 16 \\
(-3 \cdot 44 \text { to } \\
-2 \cdot 86)\end{array}$ & $\begin{array}{l}-7.54 \\
(-8.53 \text { to } \\
-6.63)\end{array}$ \\
\hline South Asia & $\begin{array}{l}166 \cdot 45 \\
(160 \cdot 83 \text { to } \\
172 \cdot 18)\end{array}$ & $\begin{array}{l}265 \cdot 95 \\
(256 \cdot 77 \text { to } \\
275 \cdot 37)\end{array}$ & $\begin{array}{l}51.54 \\
(43.79 \text { to } \\
59.81)\end{array}$ & $\begin{array}{l}-0.77 \\
(-1.10 \text { to } \\
-0.41)\end{array}$ & $\begin{array}{l}0 \cdot 02(-0 \cdot 27 \\
\text { to } 0 \cdot 31)\end{array}$ & $\begin{array}{l}-4 \cdot 96(-6 \cdot 26 \text { to } \\
-3 \cdot 69)\end{array}$ & $\begin{array}{l}-1.06 \\
(-1.30 \text { to } \\
-0.80)\end{array}$ & $\begin{array}{l}-2 \cdot 43 \\
(-2 \cdot 65 \text { to } \\
-2 \cdot 20)\end{array}$ & $\begin{array}{l}-4 \cdot 22 \\
(-5 \cdot 64 \text { to } \\
-2 \cdot 89)\end{array}$ \\
\hline Southeast Asia & $\begin{array}{l}145 \cdot 16 \\
(140 \cdot 54 \text { to } \\
148 \cdot 79)\end{array}$ & $\begin{array}{l}300 \cdot 95 \\
(289 \cdot 61 \text { to } \\
311 \cdot 88)\end{array}$ & $\begin{array}{l}41 \cdot 83 \\
(34 \cdot 22 \text { to } \\
47 \cdot 27)\end{array}$ & $\begin{array}{l}1 \cdot 59(1 \cdot 34 \\
\text { to } 1.83)\end{array}$ & $\begin{array}{l}1.42(1 \cdot 17 \\
\text { to } 1 \cdot 63)\end{array}$ & $\begin{array}{l}-4 \cdot 10(-4 \cdot 95 \text { to } \\
-3 \cdot 23)\end{array}$ & $\begin{array}{l}-0.54 \\
(-0.68 \text { to } \\
-0.42)\end{array}$ & $\begin{array}{l}0 \cdot 12(-0 \cdot 03 \\
\text { to } 0 \cdot 28)\end{array}$ & $\begin{array}{l}-3 \cdot 61 \\
(-4.42 \text { to } \\
-2 \cdot 82)\end{array}$ \\
\hline Australasia & $\begin{array}{l}6 \cdot 38(6 \cdot 17 \\
\text { to } 6 \cdot 58)\end{array}$ & $\begin{array}{l}11 \cdot 11 \\
(10.48 \text { to } \\
11 \cdot 79)\end{array}$ & $\begin{array}{l}0 \cdot 24(0 \cdot 20 \\
\text { to } 0 \cdot 27)\end{array}$ & $\begin{array}{l}-0 \cdot 23 \\
(-0 \cdot 61 \text { to } \\
0 \cdot 16)\end{array}$ & $\begin{array}{l}-1 \cdot 00 \\
(-1.37 \text { to } \\
-0.60)\end{array}$ & $\begin{array}{l}-4 \cdot 13(-5 \cdot 09 \text { to } \\
-3 \cdot 21)\end{array}$ & $\begin{array}{l}-0.22 \\
(-0.41 \text { to } \\
-0.03)\end{array}$ & $\begin{array}{l}-0.36 \\
(-0.66 \text { to } \\
-0.08)\end{array}$ & $\begin{array}{l}-3.63 \\
(-4.62 \text { to } \\
-2.62)\end{array}$ \\
\hline Caribbean & $\begin{array}{l}55 \cdot 36 \\
(53 \cdot 72 \text { to } \\
57 \cdot 02)\end{array}$ & $\begin{array}{l}70 \cdot 84 \\
(68 \cdot 52 \text { to } \\
73 \cdot 41)\end{array}$ & $\begin{array}{l}7 \cdot 19(6 \cdot 17 \\
\text { to } 9 \cdot 38)\end{array}$ & $\begin{array}{l}1.89(1.56 \\
\text { to } 2 \cdot 19)\end{array}$ & $\begin{array}{l}0 \cdot 87(0 \cdot 62 \\
\text { to } 1 \cdot 08)\end{array}$ & $\begin{array}{l}-6 \cdot 30(-7 \cdot 35 \text { to } \\
-4 \cdot 92)\end{array}$ & $\begin{array}{l}-0 \cdot 20 \\
(-0 \cdot 35 \text { to } \\
-0 \cdot 05)\end{array}$ & $\begin{array}{l}-1 \cdot 15 \\
(-1 \cdot 32 \text { to } \\
-0 \cdot 98)\end{array}$ & $\begin{array}{l}-3 \cdot 93 \\
(-4 \cdot 93 \text { to } \\
-2 \cdot 50)\end{array}$ \\
\hline Central Europe & $\begin{array}{l}26 \cdot 72 \\
(25 \cdot 97 \text { to } \\
27 \cdot 46)\end{array}$ & $\begin{array}{l}41 \cdot 97 \\
(40 \cdot 52 \text { to } \\
43 \cdot 45)\end{array}$ & $\begin{array}{l}1.69(1.57 \\
\text { to } 1.97)\end{array}$ & $\begin{array}{l}1.58(1.40 \\
\text { to } 1.75)\end{array}$ & $\begin{array}{l}0 \cdot 40(0 \cdot 26 \\
\text { to } 0 \cdot 56)\end{array}$ & $\begin{array}{l}-2.77(-3.18 \text { to } \\
-1.98)\end{array}$ & $\begin{array}{l}-1 \cdot 61 \\
(-1.74 \text { to } \\
-1 \cdot 50)\end{array}$ & $\begin{array}{l}-0 \cdot 29 \\
(-0.42 \text { to } \\
-0 \cdot 17)\end{array}$ & $\begin{array}{l}-5 \cdot 95 \\
(-6 \cdot 43 \text { to } \\
-5 \cdot 27)\end{array}$ \\
\hline Eastern Europe & $\begin{array}{l}79 \cdot 27 \\
(76 \cdot 34 \text { to } \\
82 \cdot 09)\end{array}$ & $\begin{array}{l}118 \cdot 55 \\
(113 \cdot 61 \text { to } \\
123 \cdot 59)\end{array}$ & $\begin{array}{l}7 \cdot 89(5 \cdot 14 \\
\text { to } 8 \cdot 64)\end{array}$ & $\begin{array}{l}1.33(0.99 \\
\text { to } 1.69)\end{array}$ & $\begin{array}{l}1.73(1.40 \\
\text { to } 2.03)\end{array}$ & $8.28(5.46$ to 9.09$)$ & $\begin{array}{l}-0 \cdot 58 \\
(-0 \cdot 80 \text { to } \\
-0 \cdot 39)\end{array}$ & $\begin{array}{l}-0.75 \\
(-0.97 \text { to } \\
-0.53)\end{array}$ & $\begin{array}{l}-4 \cdot 80 \\
(-7 \cdot 62 \text { to } \\
-3 \cdot 91)\end{array}$ \\
\hline Western Europe & $\begin{array}{l}10 \cdot 84 \\
(10 \cdot 52 \text { to } \\
11 \cdot 15)\end{array}$ & $\begin{array}{l}17 \cdot 25 \\
(16 \cdot 56 \text { to } \\
17 \cdot 93)\end{array}$ & $\begin{array}{l}0.54(0.50 \\
\text { to } 0 \cdot 64)\end{array}$ & $\begin{array}{l}-0 \cdot 23 \\
(-0 \cdot 39 \text { to } \\
-0 \cdot 03)\end{array}$ & $\begin{array}{l}-0.72 \\
(-0.88 \text { to } \\
-0.56)\end{array}$ & $\begin{array}{l}-4 \cdot 22(-4 \cdot 67 \text { to } \\
-3 \cdot 81)\end{array}$ & $\begin{array}{l}-1 \cdot 18 \\
(-1 \cdot 28 \text { to } \\
-1 \cdot 08)\end{array}$ & $\begin{array}{l}-0.64 \\
(-0.78 \text { to } \\
-0.52)\end{array}$ & $\begin{array}{l}-4 \cdot 88 \\
(-5 \cdot 53 \text { to } \\
-3 \cdot 78)\end{array}$ \\
\hline Andean Latin America & $\begin{array}{l}117 \cdot 89 \\
(113 \cdot 83 \text { to } \\
122 \cdot 14)\end{array}$ & $\begin{array}{l}161 \cdot 10 \\
(153 \cdot 28 \text { to } \\
168 \cdot 91)\end{array}$ & $\begin{array}{l}9 \cdot 37(8.16 \\
\text { to } 11.90)\end{array}$ & $\begin{array}{l}0.00 \\
(-0 \cdot 30 \text { to } \\
0 \cdot 33)\end{array}$ & $\begin{array}{l}-0 \cdot 89 \\
(-1 \cdot 18 \text { to } \\
-0 \cdot 60)\end{array}$ & $\begin{array}{l}-8 \cdot 25(-9 \cdot 07 \text { to } \\
-5 \cdot 88)\end{array}$ & $\begin{array}{l}-0 \cdot 81 \\
(-1.04 \text { to } \\
-0 \cdot 54)\end{array}$ & $\begin{array}{l}-0.78 \\
(-1.06 \text { to } \\
-0.52)\end{array}$ & $\begin{array}{l}-4 \cdot 86 \\
(-5 \cdot 88 \text { to } \\
-3 \cdot 78)\end{array}$ \\
\hline Central Latin America & $\begin{array}{l}32 \cdot 53 \\
(31 \cdot 73 \text { to } \\
33 \cdot 35)\end{array}$ & $\begin{array}{l}49 \cdot 40 \\
(48.00 \text { to } \\
50 \cdot 99)\end{array}$ & $\begin{array}{l}3 \cdot 38(3 \cdot 12 \\
\text { to } 4 \cdot 19)\end{array}$ & $\begin{array}{l}0.75(0.52 \\
\text { to } 0.93)\end{array}$ & $\begin{array}{l}-0 \cdot 39 \\
(-0 \cdot 61 \text { to } \\
-0 \cdot 19)\end{array}$ & $\begin{array}{l}-7 \cdot 53(-7 \cdot 85 \text { to } \\
-6 \cdot 72)\end{array}$ & $\begin{array}{l}-1.69 \\
(-1.83 \text { to } \\
-1.55)\end{array}$ & $\begin{array}{l}-1 \cdot 51 \\
(-1.67 \text { to } \\
-1 \cdot 35)\end{array}$ & $\begin{array}{l}-4 \cdot 21 \\
(-4 \cdot 74 \text { to } \\
-3 \cdot 03)\end{array}$ \\
\hline
\end{tabular}




\begin{tabular}{|c|c|c|c|c|c|c|c|c|c|}
\hline & \multirow{2}{*}{\multicolumn{3}{|c|}{$\begin{array}{l}\text { Age-standardisedrates in } 2013 \text { (per } \\
100000 \text { population) }\end{array}$}} & \multicolumn{6}{|c|}{ Annualised rate of change (\%) } \\
\hline & & & & \multicolumn{3}{|l|}{ 1990-2000 } & \multicolumn{3}{|l|}{ 2000-2013 } \\
\hline Southern Latin America & $\begin{array}{l}26 \cdot 56 \\
(25 \cdot 80 \text { to } \\
27 \cdot 32)\end{array}$ & $\begin{array}{l}38 \cdot 76 \\
(37 \cdot 10 \text { to } \\
40 \cdot 48)\end{array}$ & $\begin{array}{l}2 \cdot 05(1 \cdot 85 \\
\text { to } 2 \cdot 30)\end{array}$ & $\begin{array}{l}1.43(1.06 \\
\text { to } 1.78)\end{array}$ & $\begin{array}{l}-0 \cdot 06 \\
(-0.35 \text { to } \\
0.23)\end{array}$ & $\begin{array}{l}-4 \cdot 77(-5 \cdot 78 \text { to } \\
-4 \cdot 23)\end{array}$ & $\begin{array}{l}-2.56 \\
(-2.75 \text { to } \\
-2.39)\end{array}$ & $\begin{array}{l}-1.56 \\
(-1.78 \text { to } \\
-1.32)\end{array}$ & $\begin{array}{l}-3 \cdot 35 \\
(-4.03 \text { to } \\
-2 \cdot 70)\end{array}$ \\
\hline $\begin{array}{l}\text { North Africa and Middle } \\
\text { East }\end{array}$ & $\begin{array}{l}34.45 \\
(33.65 \text { to } \\
35 \cdot 29)\end{array}$ & $\begin{array}{l}48 \cdot 00 \\
(46 \cdot 50 \text { to } \\
49 \cdot 55)\end{array}$ & $\begin{array}{l}4 \cdot 56(4 \cdot 10 \\
\text { to } 5 \cdot 37)\end{array}$ & $\begin{array}{l}1 \cdot 07(0 \cdot 85 \\
\text { to } 1 \cdot 30)\end{array}$ & $\begin{array}{l}-0.32 \\
(-0.50 \text { to } \\
-0.12)\end{array}$ & $\begin{array}{l}-3 \cdot 72(-4 \cdot 35 \text { to } \\
-3 \cdot 07)\end{array}$ & $\begin{array}{l}-1 \cdot 10 \\
(-1 \cdot 18 \text { to } \\
-1 \cdot 03)\end{array}$ & $\begin{array}{l}-1 \cdot 26 \\
(-1.35 \text { to } \\
-1 \cdot 16)\end{array}$ & $\begin{array}{l}-4 \cdot 58 \\
(-5 \cdot 34 \text { to } \\
-3 \cdot 89)\end{array}$ \\
\hline Oceania & $\begin{array}{l}109 \cdot 58 \\
(106 \cdot 38 \text { to } \\
112 \cdot 83)\end{array}$ & $\begin{array}{l}174.93 \\
(167.73 \text { to } \\
182.77)\end{array}$ & $\begin{array}{l}20 \cdot 21 \\
(12 \cdot 67 \text { to } \\
40 \cdot 27)\end{array}$ & $\begin{array}{l}-0 \cdot 73 \\
(-1 \cdot 12 \text { to } \\
-0 \cdot 36)\end{array}$ & $\begin{array}{l}-0.85 \\
(-1.20 \text { to } \\
-0.52)\end{array}$ & $\begin{array}{l}-6 \cdot 29(-8 \cdot 55 \text { to } \\
-2 \cdot 59)\end{array}$ & $\begin{array}{l}0.77(0 \cdot 60 \\
\text { to } 0.98)\end{array}$ & $\begin{array}{l}0 \cdot 14(-0 \cdot 09 \\
\text { to } 0 \cdot 37)\end{array}$ & $\begin{array}{l}-2 \cdot 96 \\
(-4.58 \text { to } \\
-0.91)\end{array}$ \\
\hline Central sub-Saharan Africa & $\begin{array}{l}285.76 \\
(271.43 \text { to } \\
299.93)\end{array}$ & $\begin{array}{l}485 \cdot 43 \\
(453 \cdot 81 \text { to } \\
520 \cdot 51)\end{array}$ & $\begin{array}{l}100 \cdot 60 \\
(79.63 \text { to } \\
118 \cdot 71)\end{array}$ & $\begin{array}{l}0.63(0 \cdot 14 \\
\text { to } 1 \cdot 09)\end{array}$ & $\begin{array}{l}0 \cdot 31(-0 \cdot 12 \\
\text { to } 0 \cdot 74)\end{array}$ & $-0 \cdot 12(-1 \cdot 35$ to $1 \cdot 27)$ & $\begin{array}{l}0 \cdot 07 \\
(-0 \cdot 12 \text { to } \\
0 \cdot 28)\end{array}$ & $\begin{array}{l}-0 \cdot 17 \\
(-0 \cdot 41 \text { to } \\
0 \cdot 06)\end{array}$ & $\begin{array}{l}-3.41 \\
(-4.67 \text { to } \\
-1.99)\end{array}$ \\
\hline Eastern sub-Saharan Africa & $\begin{array}{l}203.95 \\
(191.93 \text { to } \\
217.39)\end{array}$ & $\begin{array}{l}311.05 \\
(290.44 \text { to } \\
333.89)\end{array}$ & $\begin{array}{l}96 \cdot 61 \\
(82 \cdot 38 \text { to } \\
104 \cdot 22)\end{array}$ & $\begin{array}{l}-0.99 \\
(-1.42 \text { to } \\
-0.54)\end{array}$ & $\begin{array}{l}-1.00 \\
(-1.43 \text { to } \\
-0.54)\end{array}$ & $-0.67(-1.82$ to 0.09$)$ & $\begin{array}{l}-0.34 \\
(-0.52 \text { to } \\
-0 \cdot 16)\end{array}$ & $\begin{array}{l}-0.38 \\
(-0.57 \text { to } \\
-0.20)\end{array}$ & $\begin{array}{l}-3 \cdot 08 \\
(-4 \cdot 12 \text { to } \\
-2 \cdot 40)\end{array}$ \\
\hline Southern sub-Saharan Africa & $\begin{array}{l}719 \cdot 33 \\
(642 \cdot 13 \text { to } \\
823 \cdot 58)\end{array}$ & $\begin{array}{l}942 \cdot 17 \\
(834 \cdot 80 \text { to } \\
1,079 \cdot 88)\end{array}$ & $\begin{array}{l}62 \cdot 50 \\
(53 \cdot 16 \text { to } \\
71 \cdot 44)\end{array}$ & $\begin{array}{l}-4 \cdot 74 \\
(-5 \cdot 78 \text { to } \\
-3 \cdot 50)\end{array}$ & $\begin{array}{l}-3 \cdot 70 \\
(-4 \cdot 66 \text { to } \\
-2 \cdot 54)\end{array}$ & $1 \cdot 34(-0 \cdot 22$ to $2 \cdot 77)$ & $\begin{array}{l}0 \cdot 14 \\
(-0 \cdot 26 \text { to } \\
0 \cdot 54)\end{array}$ & $\begin{array}{l}-0 \cdot 09 \\
(-0.41 \text { to } \\
0 \cdot 28)\end{array}$ & $\begin{array}{l}-4 \cdot 12 \\
(-5.91 \text { to } \\
-2.91)\end{array}$ \\
\hline
\end{tabular}

Data in parentheses are $95 \%$ uncertainty intervals. 


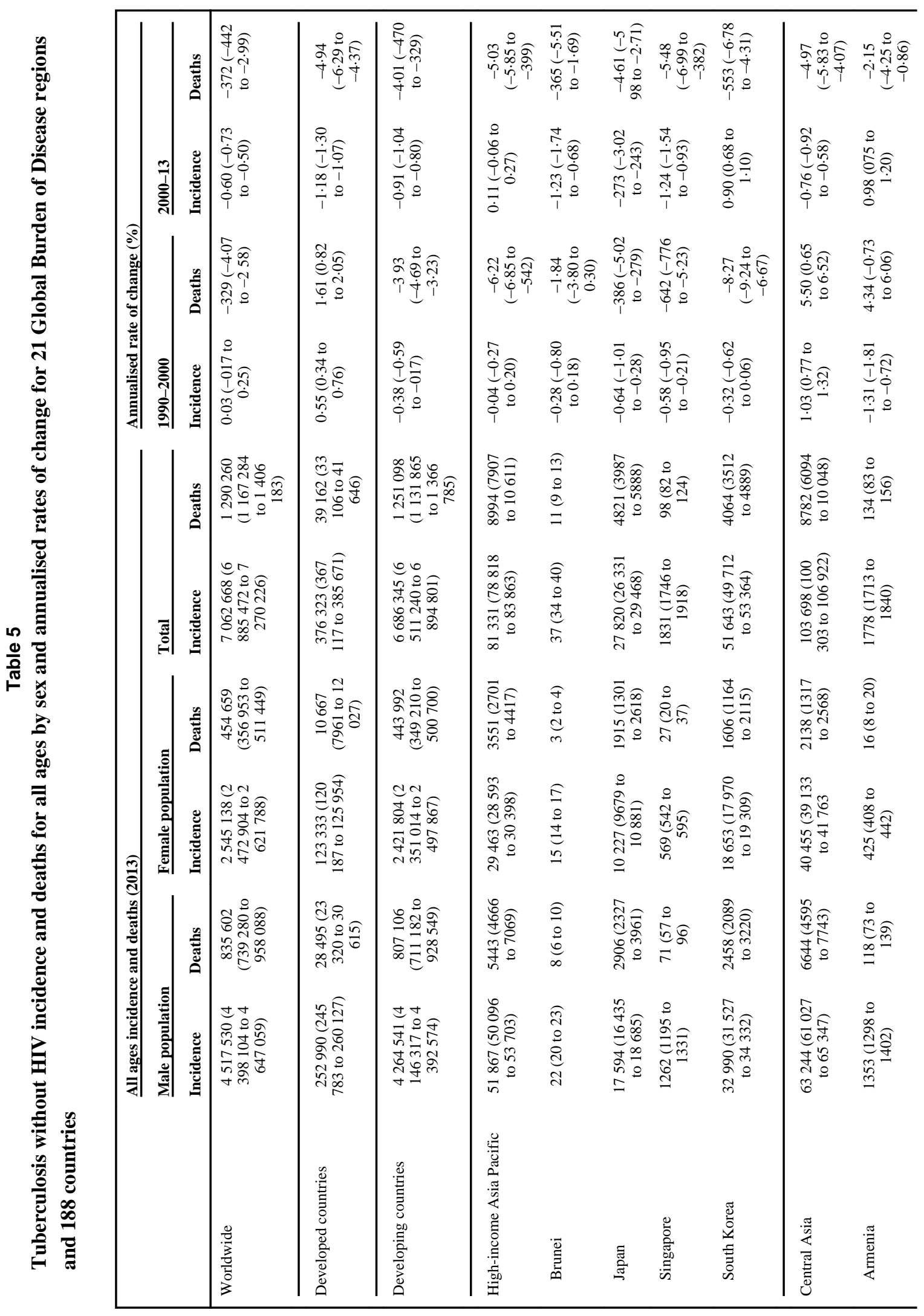




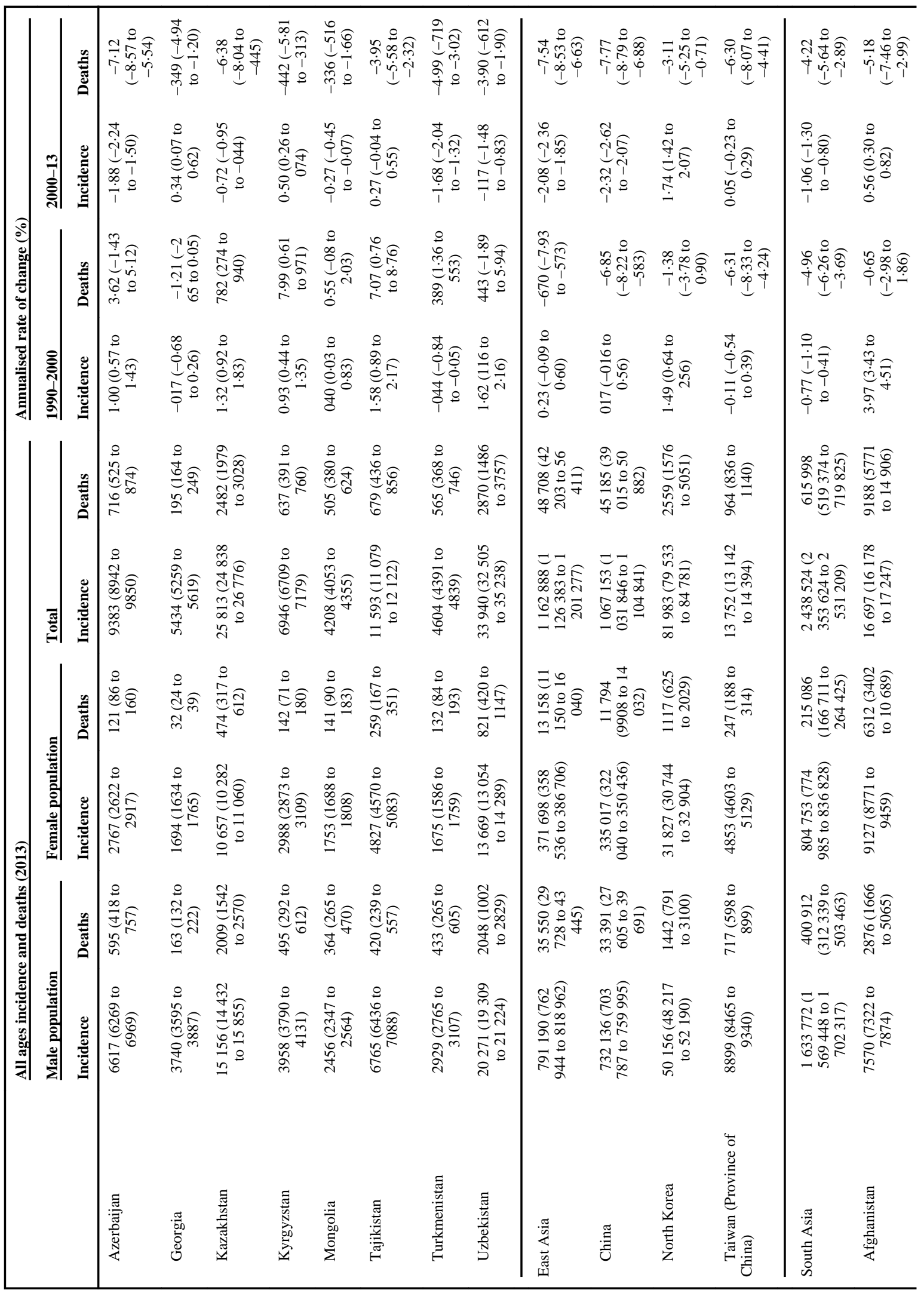




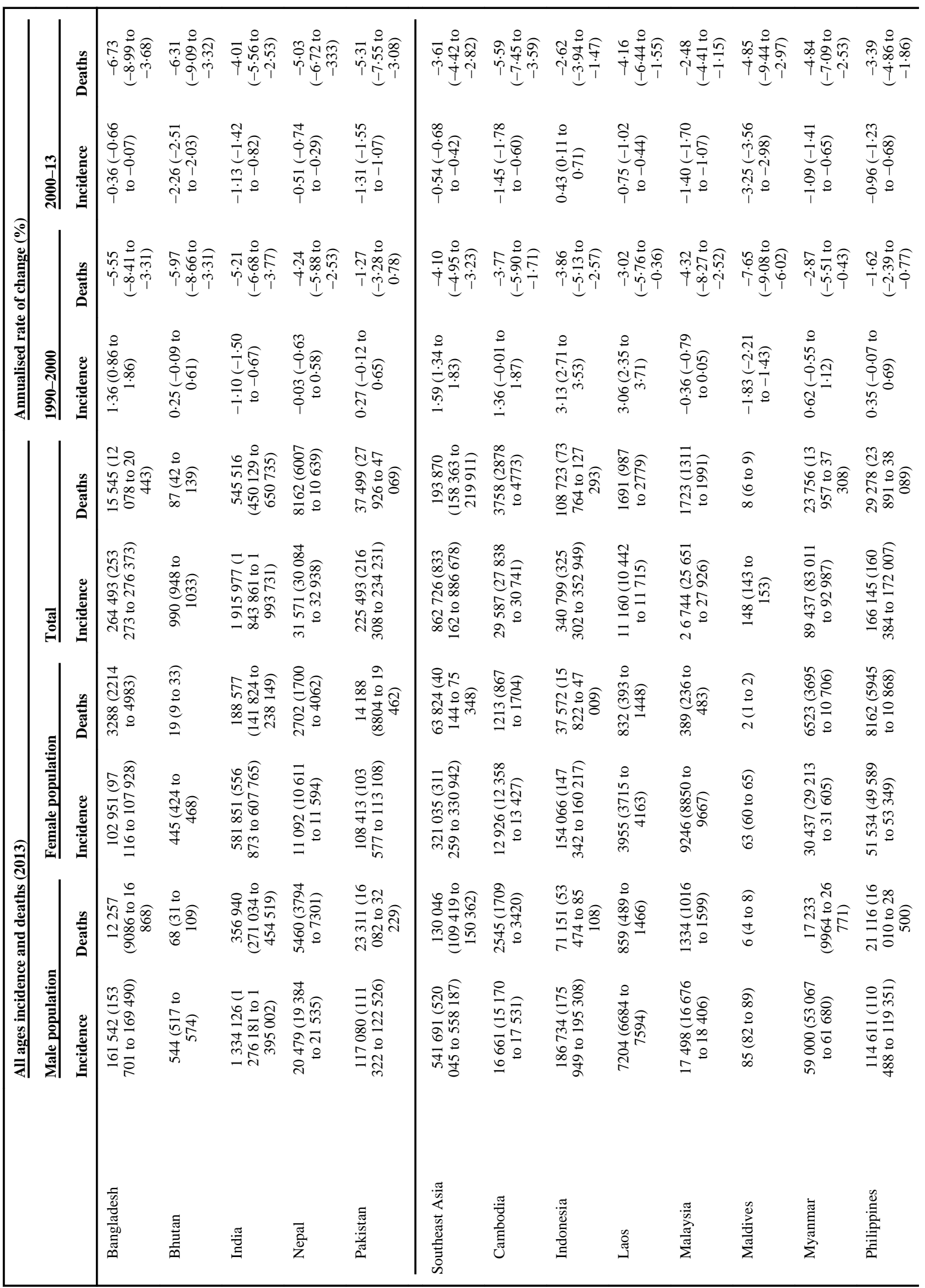




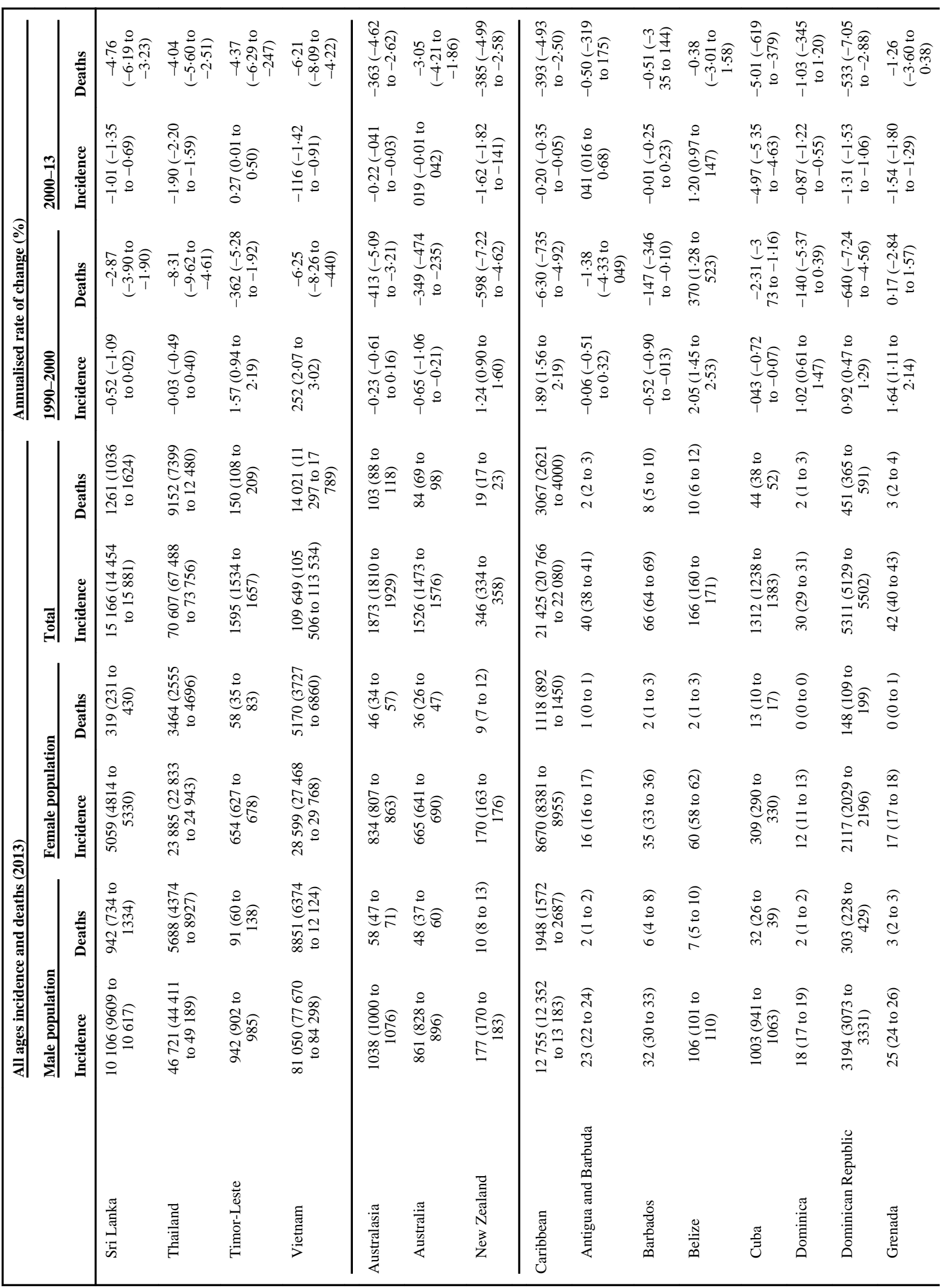




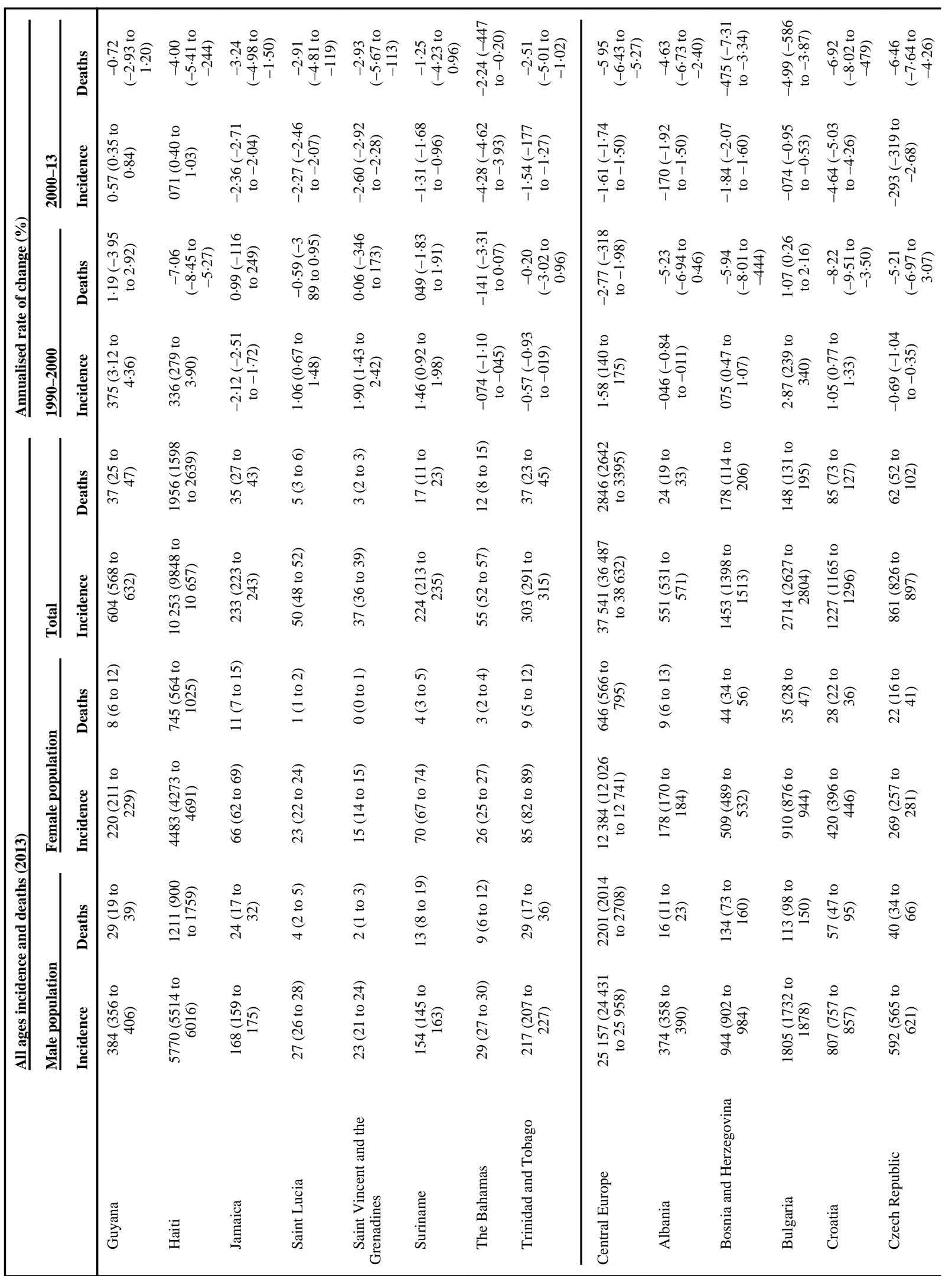




\begin{tabular}{|c|c|c|c|c|c|c|c|c|c|c|c|c|c|c|c|}
\hline \multirow{2}{*}{\multicolumn{2}{|c|}{$\mid$}} & 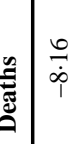 & के & 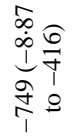 & 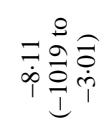 & 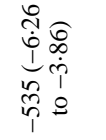 & 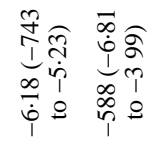 & 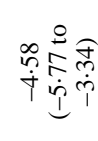 & 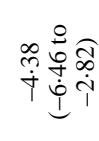 & 웜 & 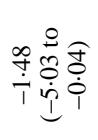 & 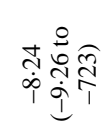 & 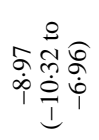 & 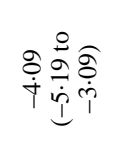 & 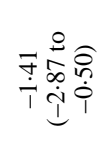 \\
\hline & & 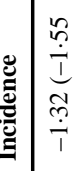 & : & 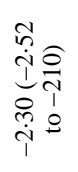 & 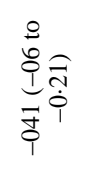 & 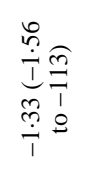 & 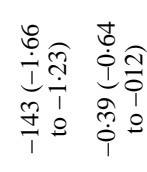 & 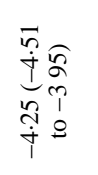 & 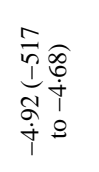 & 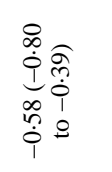 & 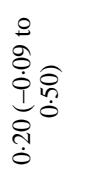 & $\underset{\substack{0 \\
\stackrel{0}{0}}}{\substack{0 \\
0}}$ & 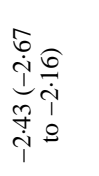 & 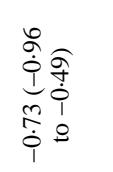 & 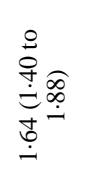 \\
\hline ब & 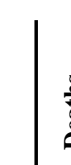 & & $\begin{array}{l}\dot{0} \\
\dot{\partial} \\
\dot{i} \\
\dot{i}\end{array}$ & & 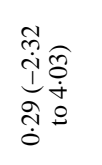 & 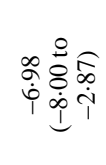 & 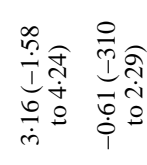 & ํํำ & 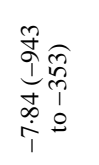 & 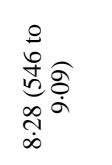 & 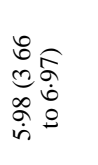 & 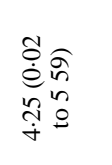 & 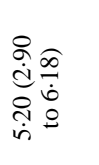 & 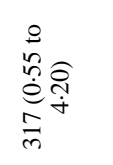 & 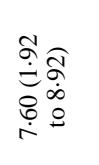 \\
\hline |⿹丁口⿹ & 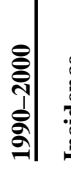 & 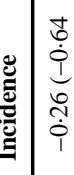 & $\begin{array}{l}0 \\
0 \\
0 \\
0\end{array}$ & 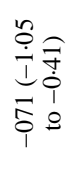 & 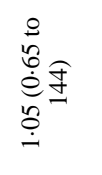 & 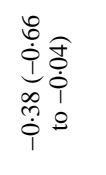 & 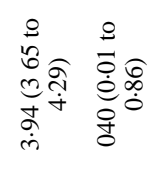 & 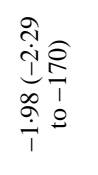 & 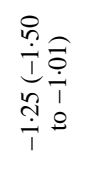 & 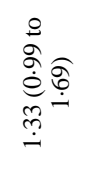 & 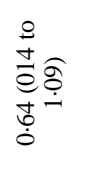 & 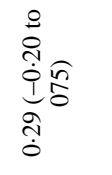 & 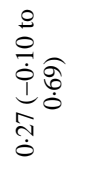 & 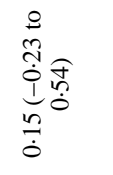 & 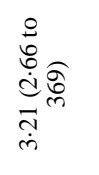 \\
\hline & 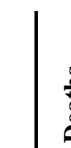 & 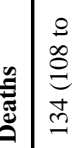 & : & 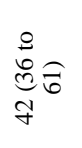 & $\begin{array}{l}\hat{f} \\
\stackrel{d}{d} \\
\stackrel{d}{d}\end{array}$ & 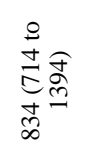 & 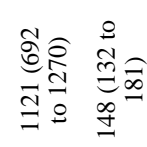 & 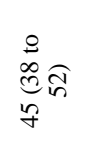 & 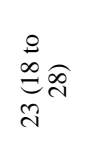 & 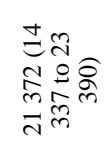 & 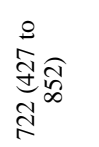 & 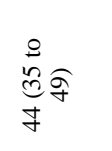 & 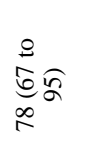 & 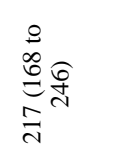 & 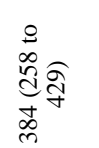 \\
\hline & : & 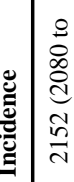 & तิ & 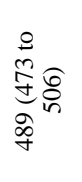 & 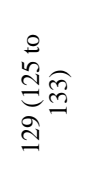 & 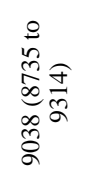 & 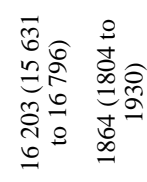 & 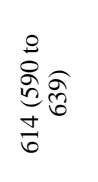 & 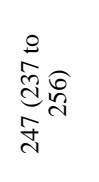 & 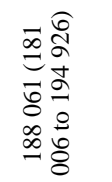 & 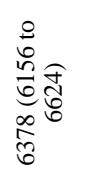 & 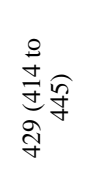 & 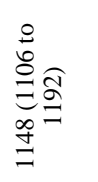 & 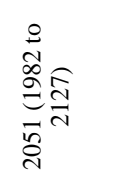 & 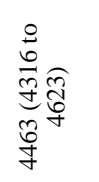 \\
\hline & & 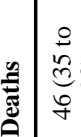 & 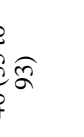 & $\begin{array}{l}\stackrel{6}{6} \\
\stackrel{0}{\infty} \\
\stackrel{\infty}{=}\end{array}$ & 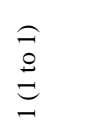 & 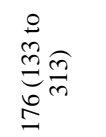 & 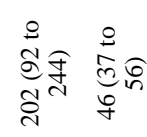 & $\stackrel{8}{\stackrel{0}{\partial} \sigma}$ & $\begin{array}{l}0 \\
0 \\
0 \\
\infty \\
=\end{array}$ & 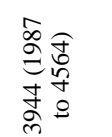 & 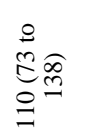 & $\begin{array}{l}\hat{\sigma} \\
\frac{0}{0} \\
0 \\
\infty \\
\infty\end{array}$ & 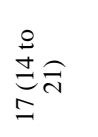 & 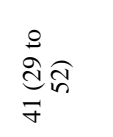 & 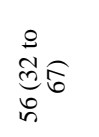 \\
\hline$\hat{3}$ & 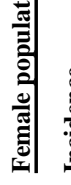 & 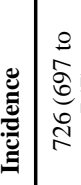 & an & $\underset{\stackrel{8}{\Xi}}{\stackrel{8}{\Xi}}$ & 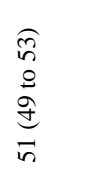 & 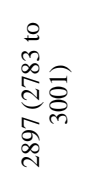 & 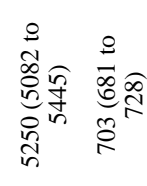 & 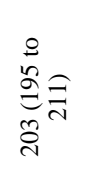 & 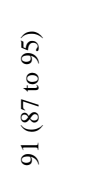 & 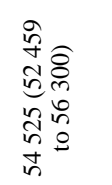 & 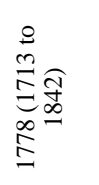 & 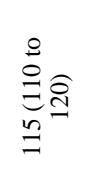 & 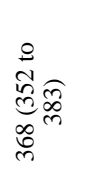 & 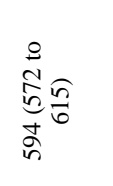 & 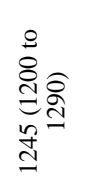 \\
\hline 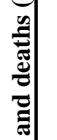 & & 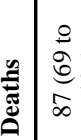 & 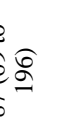 & $\underset{\substack{d \\
d}}{\stackrel{8}{y}}$ & 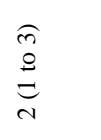 & 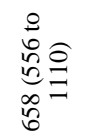 & 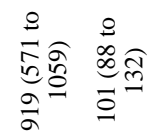 & $\underset{m}{\stackrel{0}{a}}$ & 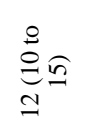 & 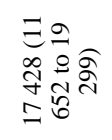 & 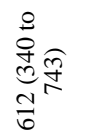 & 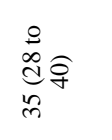 & $\underset{0}{\stackrel{0}{0}}$ & 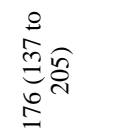 & 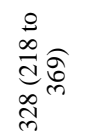 \\
\hline 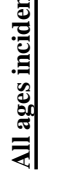 & : & 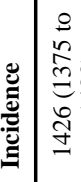 & 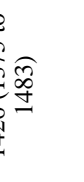 & $\begin{array}{l}0 \\
\text { वे̃ } \\
\text { तै } \\
\text { ल }\end{array}$ & 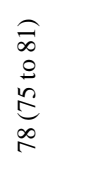 & 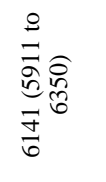 & 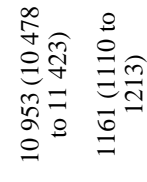 & 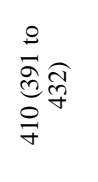 & 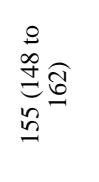 & 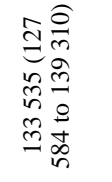 & 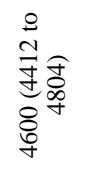 & 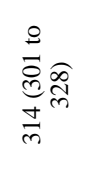 & 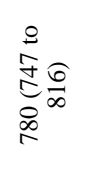 & 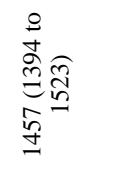 & 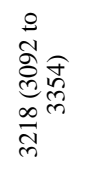 \\
\hline & & 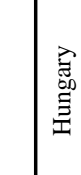 & 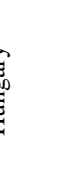 & 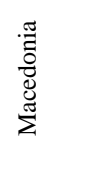 & 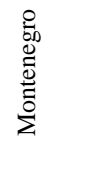 & $\begin{array}{l}\vec{z} \\
\frac{\vec{J}}{0} \\
2\end{array}$ & 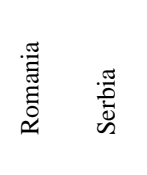 & 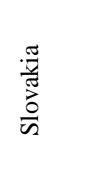 & 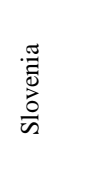 & 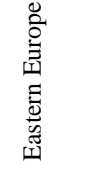 & 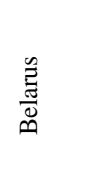 & $\begin{array}{l}\text { 㺃 } \\
\text { 总 }\end{array}$ & : & 茎 & $\frac{\tilde{a}}{\frac{0}{0}}$ \\
\hline
\end{tabular}




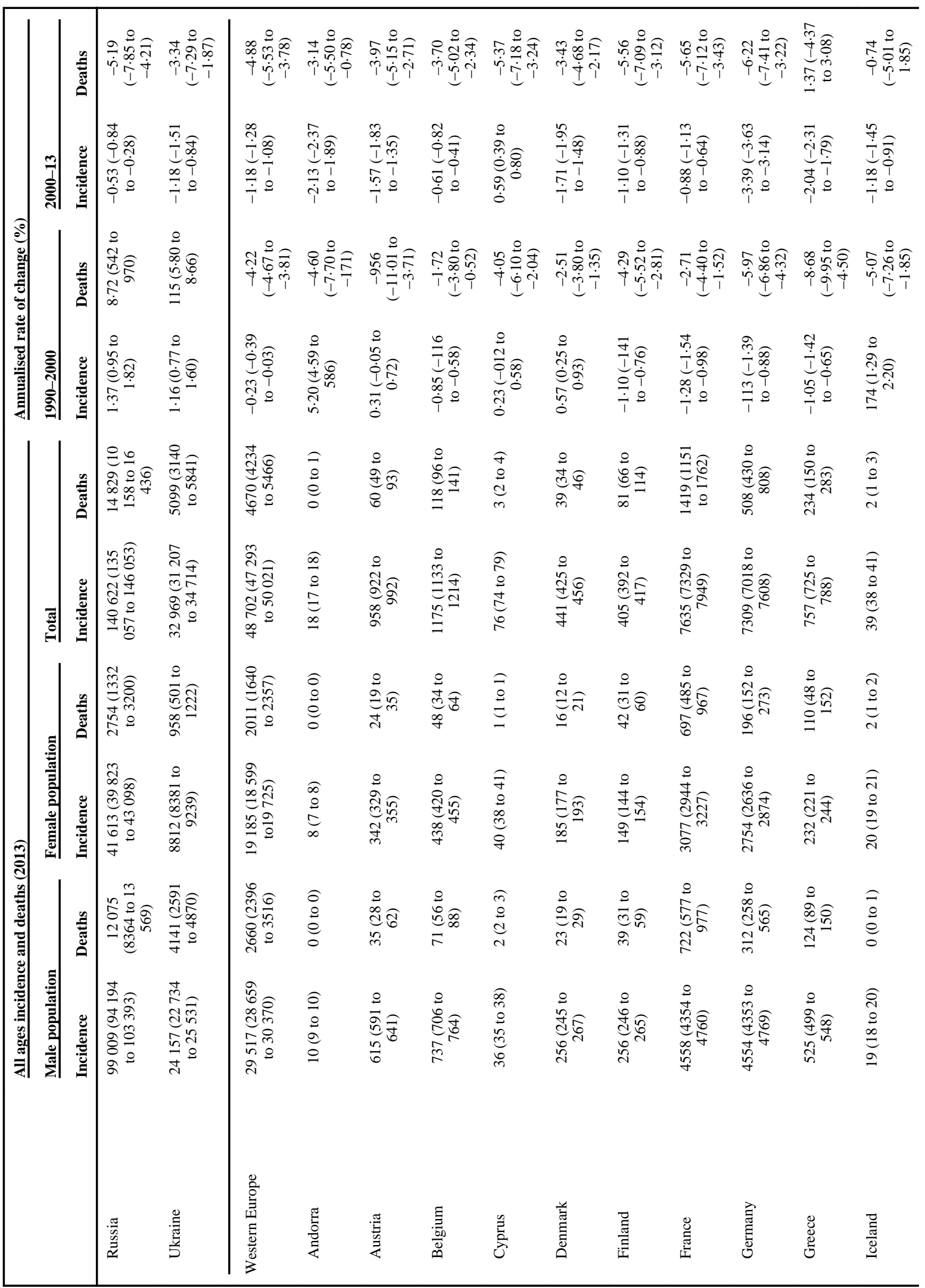




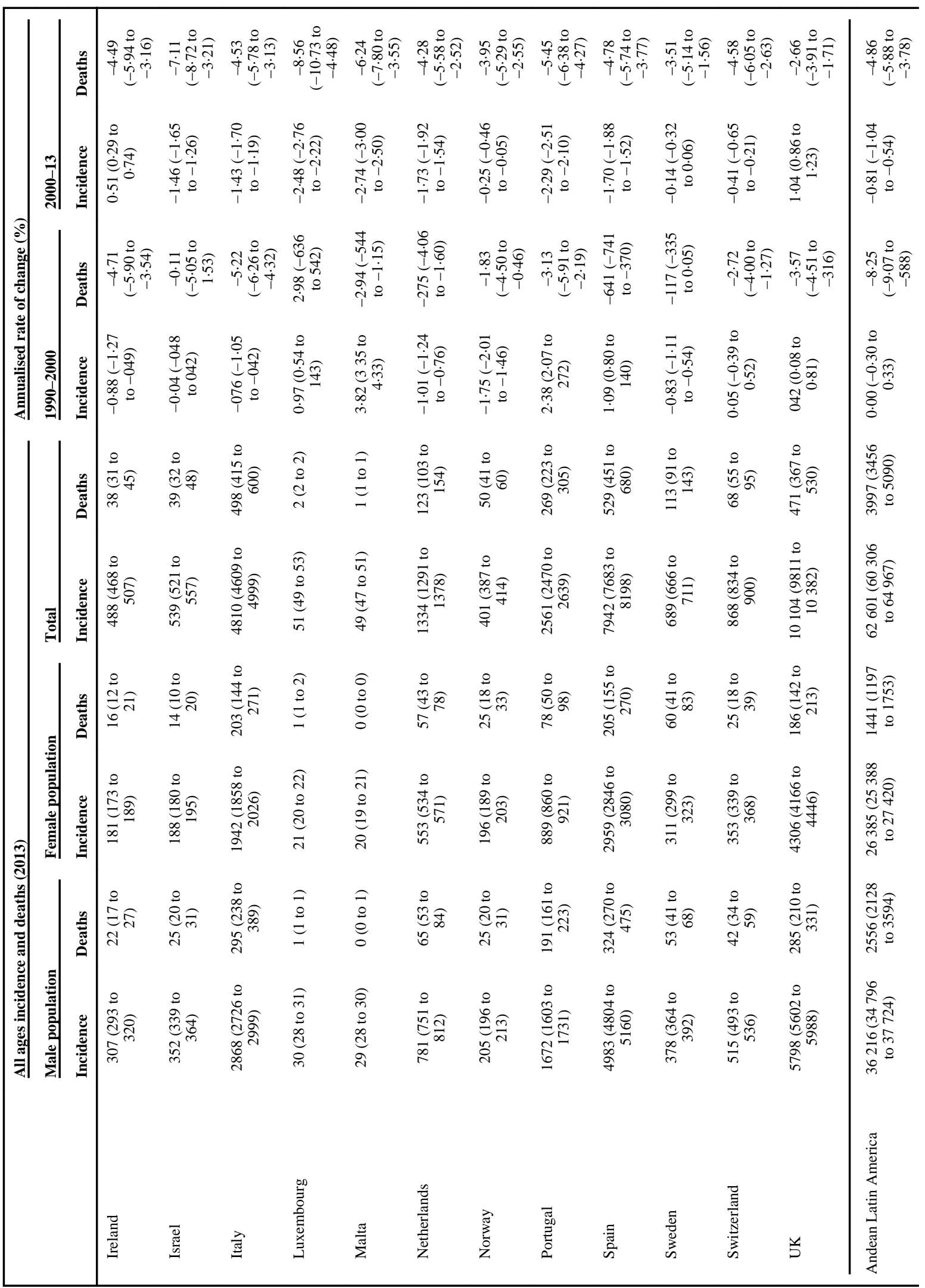




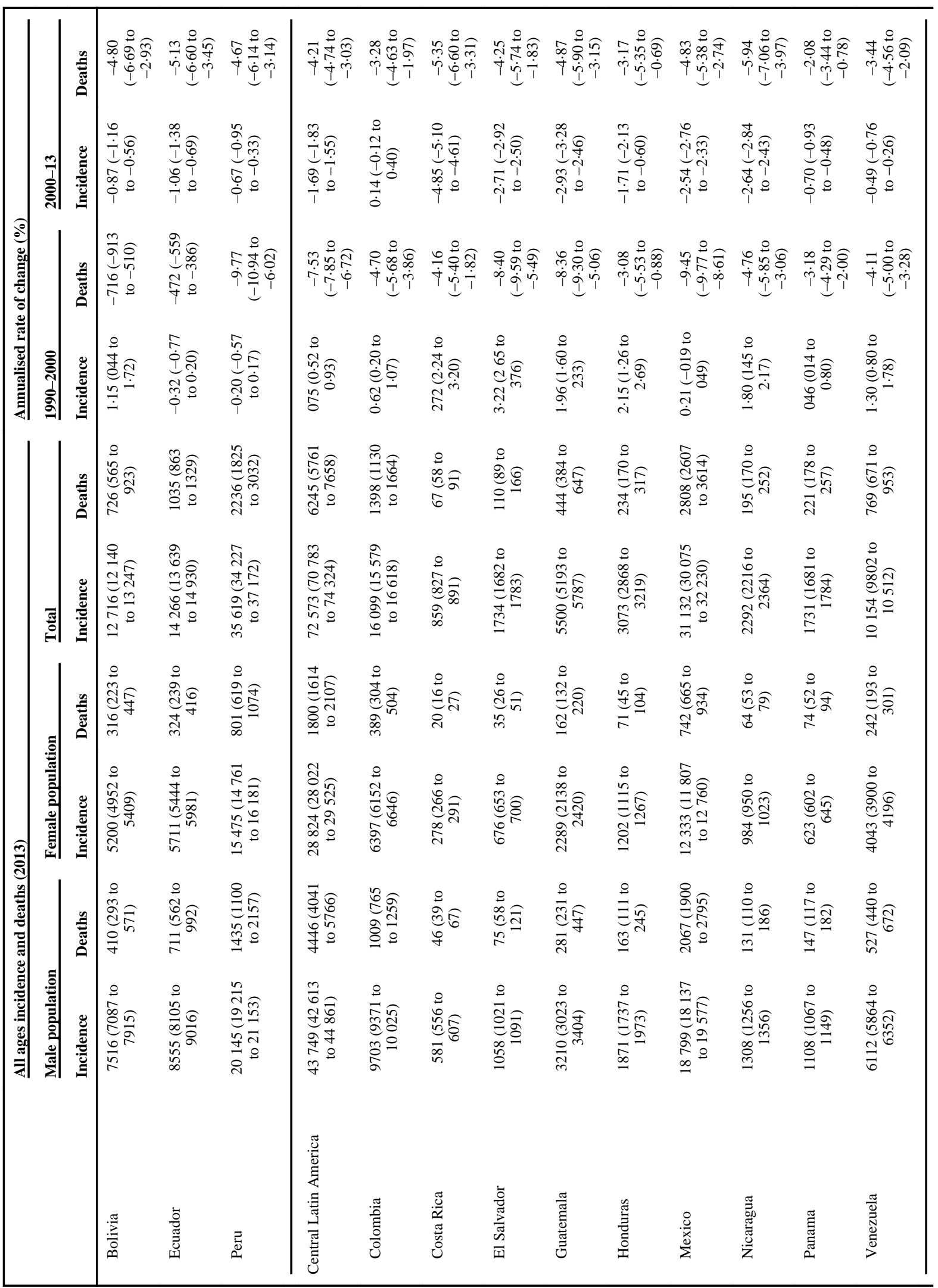




\begin{tabular}{|c|c|c|c|c|c|c|c|c|c|c|c|c|c|c|c|}
\hline & & 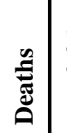 & 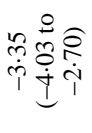 & 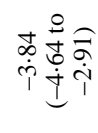 & 突突字 & 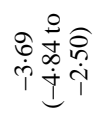 & 勇总奇 & 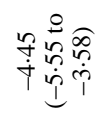 & 辛栾 & 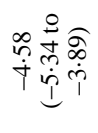 & 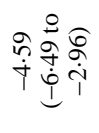 & 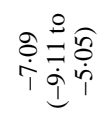 & 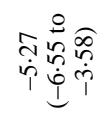 & 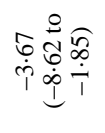 & 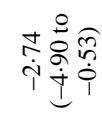 \\
\hline & 訔 & 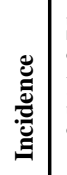 & 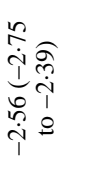 & 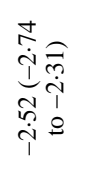 & 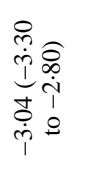 & 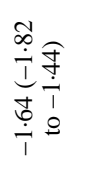 & 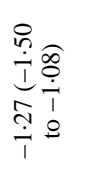 & 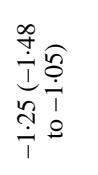 & 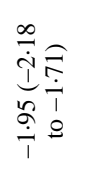 & 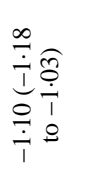 & 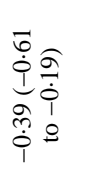 & 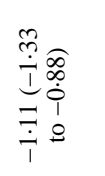 & 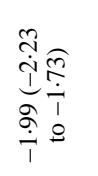 & 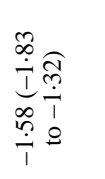 & $\begin{array}{l}\stackrel{8}{ \pm} \\
\dot{\theta} \\
\dot{e} \\
\text { to } \\
0\end{array}$ \\
\hline : & . & 号 & 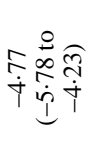 & 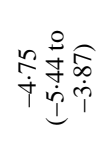 & 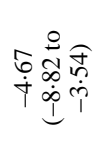 & 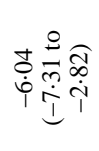 & 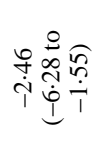 & 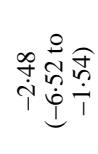 & 胥 & 总总 & 突 & 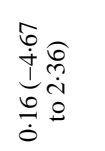 & 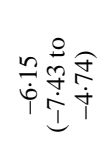 & 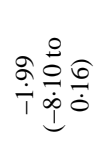 & 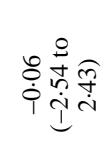 \\
\hline 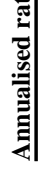 & \begin{tabular}{l} 
: \\
\multirow{1}{0}{} \\
$\vdots$ \\
$\vdots$
\end{tabular} & 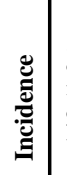 & 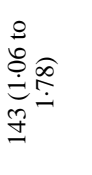 & 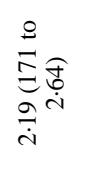 & 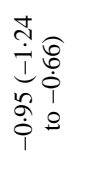 & 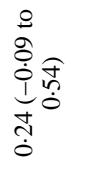 & 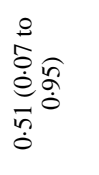 & 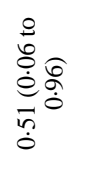 & 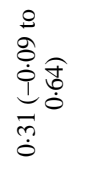 & 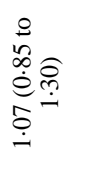 & 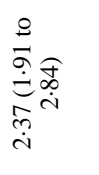 & 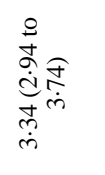 & 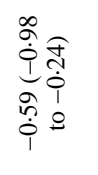 & 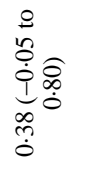 & 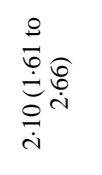 \\
\hline & & 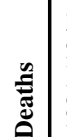 & 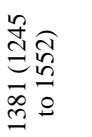 & 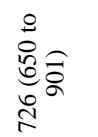 & 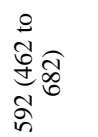 & 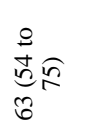 & 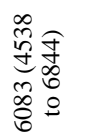 & 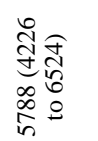 & 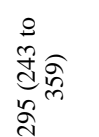 & 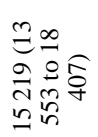 & 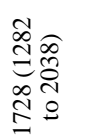 & $\begin{array}{l}a \\
0 \\
0 \\
0 \\
a\end{array}$ & 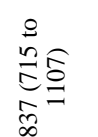 & 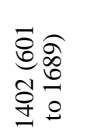 & 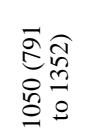 \\
\hline & 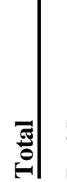 & 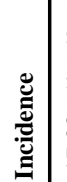 & 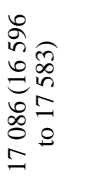 & 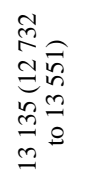 & 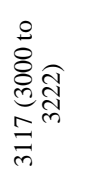 & 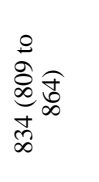 & 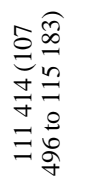 & 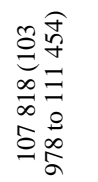 & 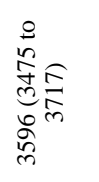 & 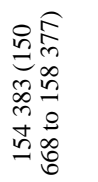 & 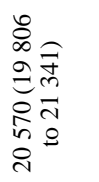 & 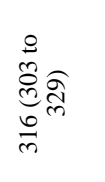 & 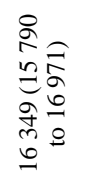 & 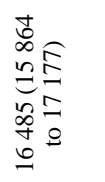 & 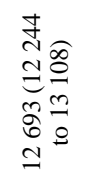 \\
\hline & & : & 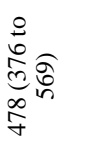 & 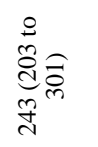 & 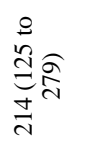 & 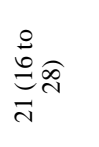 & 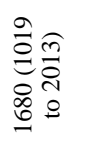 & 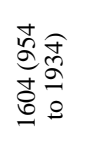 & 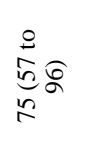 & 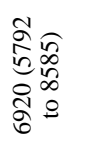 & 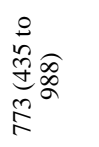 & $\begin{array}{l}\hat{\sigma} \\
\stackrel{2}{d} \\
\stackrel{d}{m}\end{array}$ & 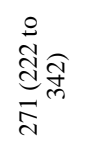 & 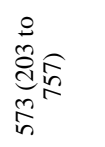 & 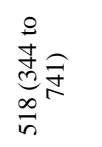 \\
\hline 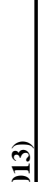 & 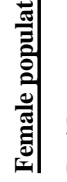 & 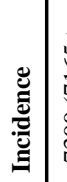 & 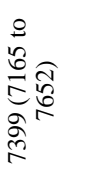 & 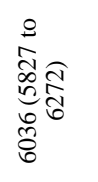 & 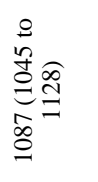 & 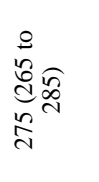 & 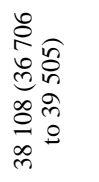 & 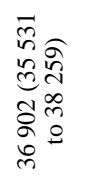 & 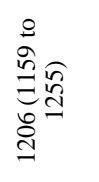 & 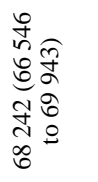 & 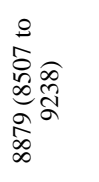 & $\begin{array}{l}\hat{\sigma} \\
\frac{0}{0} \\
o \\
o \\
o \\
o \\
0\end{array}$ & 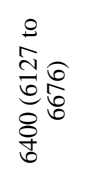 & 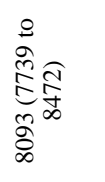 & 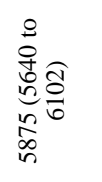 \\
\hline 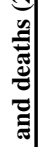 & 1 & 象 & 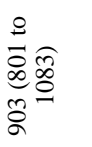 & 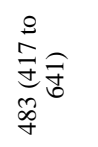 & 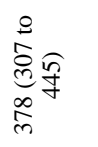 & 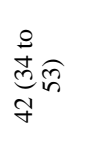 & 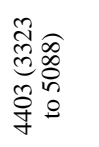 & 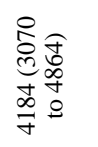 & 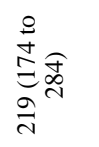 & 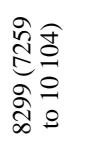 & 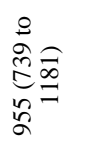 & $\begin{array}{l}\hat{0} \\
0 \\
0 \\
0 \\
0\end{array}$ & 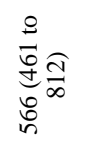 & 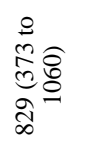 & 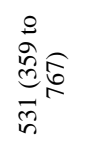 \\
\hline 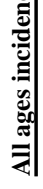 & 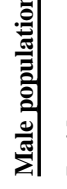 & $\begin{array}{l} \\
\stackrel{\Xi}{\Xi} \\
\stackrel{\Xi}{\Xi} \\
\stackrel{\Xi}{\Xi} \\
\end{array}$ & 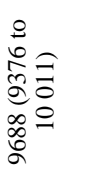 & 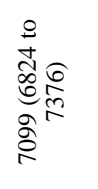 & 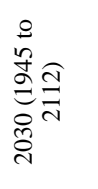 & 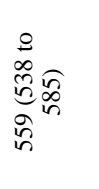 & 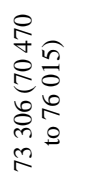 & 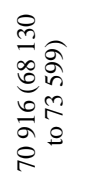 & 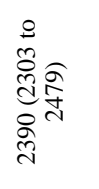 & 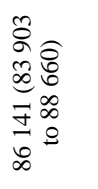 & 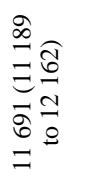 & 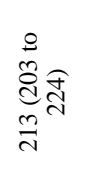 & 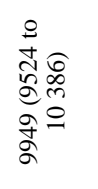 & 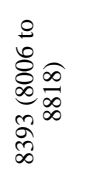 & 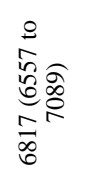 \\
\hline & & & 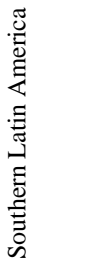 & 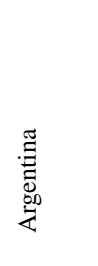 & 号 & 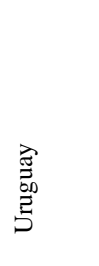 & 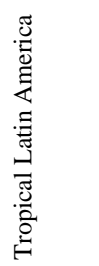 & 胥 & 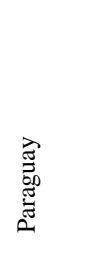 & 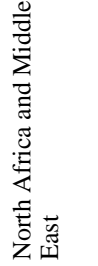 & $\begin{array}{l}\frac{\pi}{\vec{g}_{0}} \\
\frac{\mathrm{g}}{\mathbb{Z}}\end{array}$ & 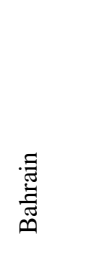 & 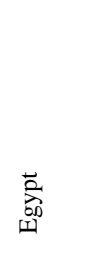 & 駦 & $\mathscr{\mathscr { F }}$ \\
\hline
\end{tabular}




\begin{tabular}{|c|c|c|c|c|c|c|c|c|c|c|c|c|c|c|c|}
\hline & & 号 & 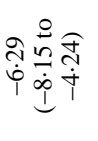 & 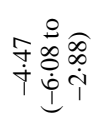 & 总蛋 & 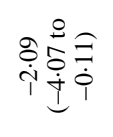 & 象孛旁 & 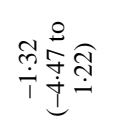 & 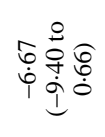 & 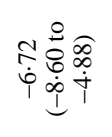 & 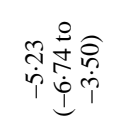 & 总总 & 导总命 & 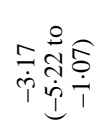 & 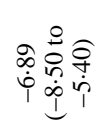 \\
\hline & : & 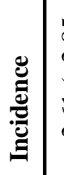 & 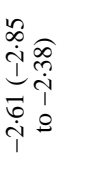 & 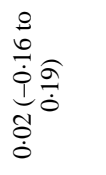 & 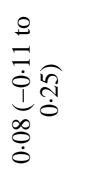 & 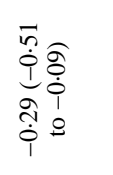 & 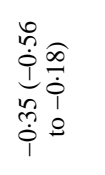 & $\begin{array}{l}\stackrel{m}{\dot{T}} \widehat{i} \\
\dot{i} \dot{i} \\
\stackrel{i}{\dot{i}}=\end{array}$ & 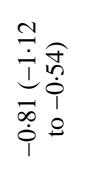 & 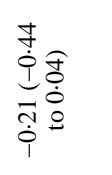 & 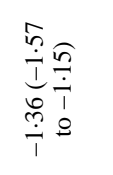 & 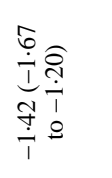 & 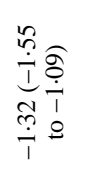 & 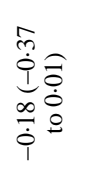 & $\begin{array}{l}\infty \\
\infty \\
0 \\
1 \\
1 \\
0 \\
0 \\
0 \\
0 \\
0 \\
0\end{array}$ \\
\hline $\begin{array}{l}0 \\
0 \\
0 \\
0 \\
0\end{array}$ & 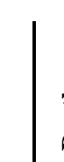 & | & 1 & 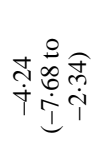 & 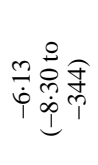 & 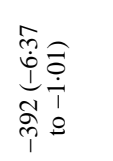 & 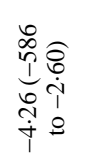 & 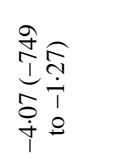 & 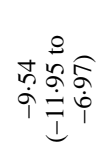 & 충 & 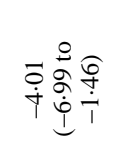 & مُ & 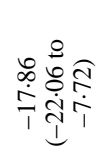 & 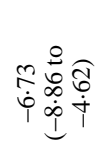 & 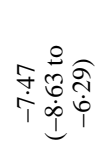 \\
\hline 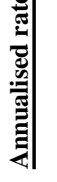 & : & 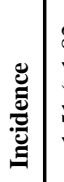 & 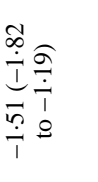 & 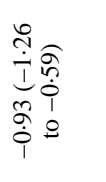 & 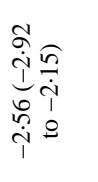 & 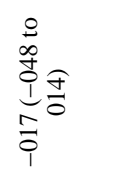 & 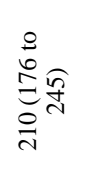 & 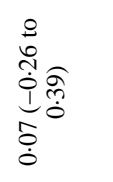 & 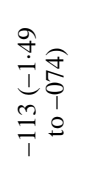 & 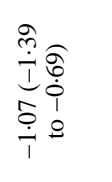 & 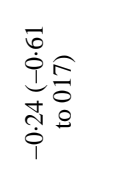 & 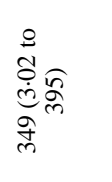 & 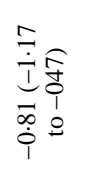 & 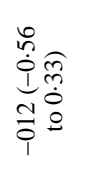 & 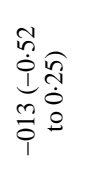 \\
\hline 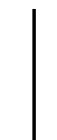 & & 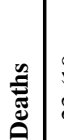 & 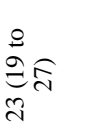 & 离 & 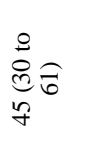 & 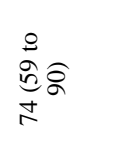 & 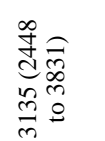 & 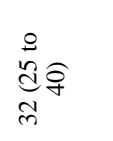 & \begin{tabular}{l}
$\infty$ \\
0 \\
0 \\
0 \\
\multirow{\sigma}{\sigma}{}
\end{tabular} & $\begin{array}{l}6 \\
0 \\
0 \\
\text { d } \\
\text { n. }\end{array}$ & $\begin{array}{l}0 \\
\text { : } \\
\text { co: } \\
\text { to } \\
\infty\end{array}$ & 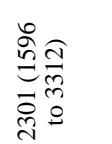 & 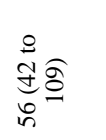 & 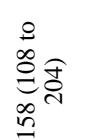 & 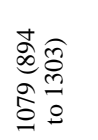 \\
\hline & 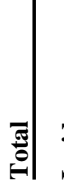 & 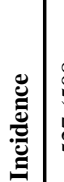 & 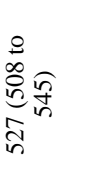 & 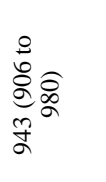 & 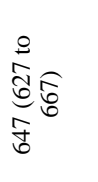 & 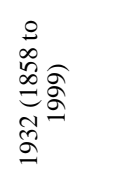 & 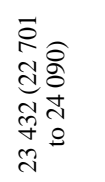 & 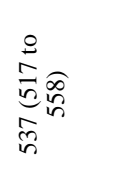 & 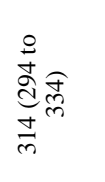 & 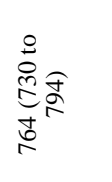 & 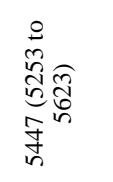 & 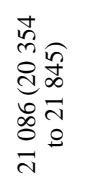 & 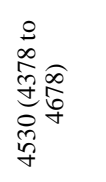 & 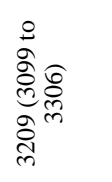 & 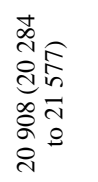 \\
\hline & & |ص & 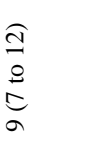 & $\begin{array}{l}\frac{6}{2} \\
\infty \\
\infty \\
\simeq\end{array}$ & $\stackrel{\stackrel{0}{C}}{\stackrel{\infty}{\infty}}$ & 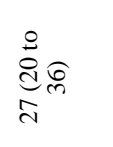 & 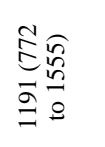 & $\begin{array}{l}\sqrt{n} \\
0 \\
\infty \\
= \\
=\end{array}$ & 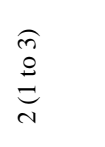 & $\stackrel{\overparen{g}}{\stackrel{\theta}{\Xi}}$ & 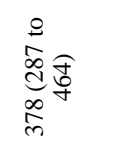 & 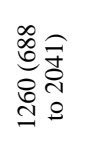 & 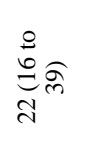 & 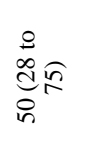 & 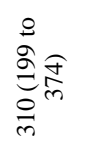 \\
\hline 3 & $\begin{array}{l}\text { : } \\
\text { : }\end{array}$ & 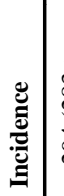 & 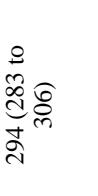 & 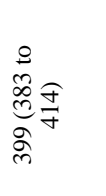 & 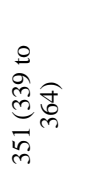 & 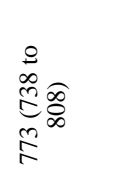 & 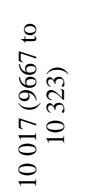 & 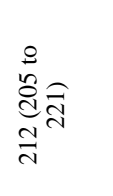 & $\begin{array}{l}\widehat{a} \\
\stackrel{0}{0} \\
\tilde{\sigma} \\
\stackrel{0}{0}\end{array}$ & 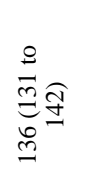 & 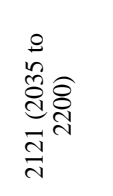 & 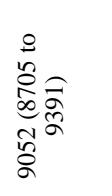 & 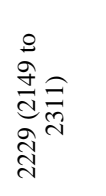 & 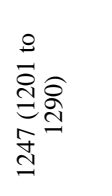 & 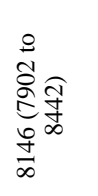 \\
\hline 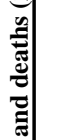 & . & 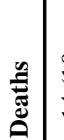 & 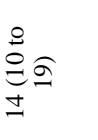 & 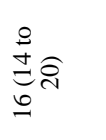 & 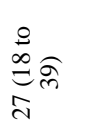 & 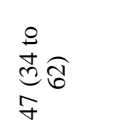 & 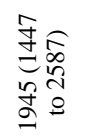 & 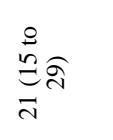 & $\begin{array}{l}0 \\
0 \\
0 \\
0 \\
0 \\
m\end{array}$ & $\begin{array}{l}n \\
0 \\
0 \\
0 \\
y\end{array}$ & 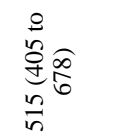 & 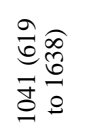 & 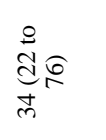 & 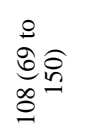 & 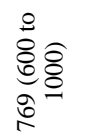 \\
\hline 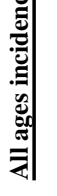 & $\begin{array}{l}\text { : } \\
\text { : }\end{array}$ & 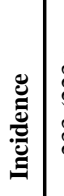 & 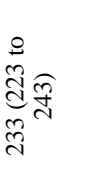 & 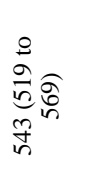 & 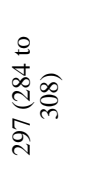 & 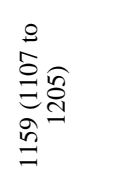 & 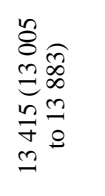 & 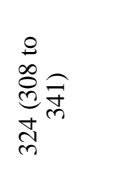 & 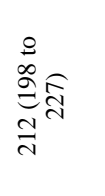 & 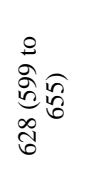 & 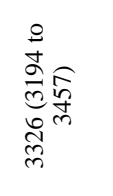 & 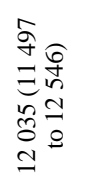 & 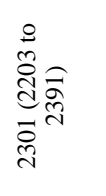 & 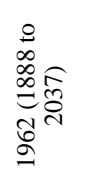 & 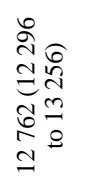 \\
\hline & & & 㞼 & 䓂 & 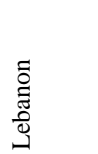 & 茹 & 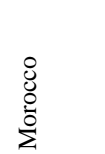 & 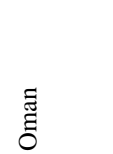 & 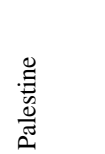 & 志 & 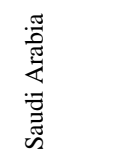 & 胥 & 苟 & 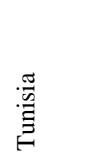 & 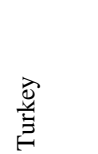 \\
\hline
\end{tabular}




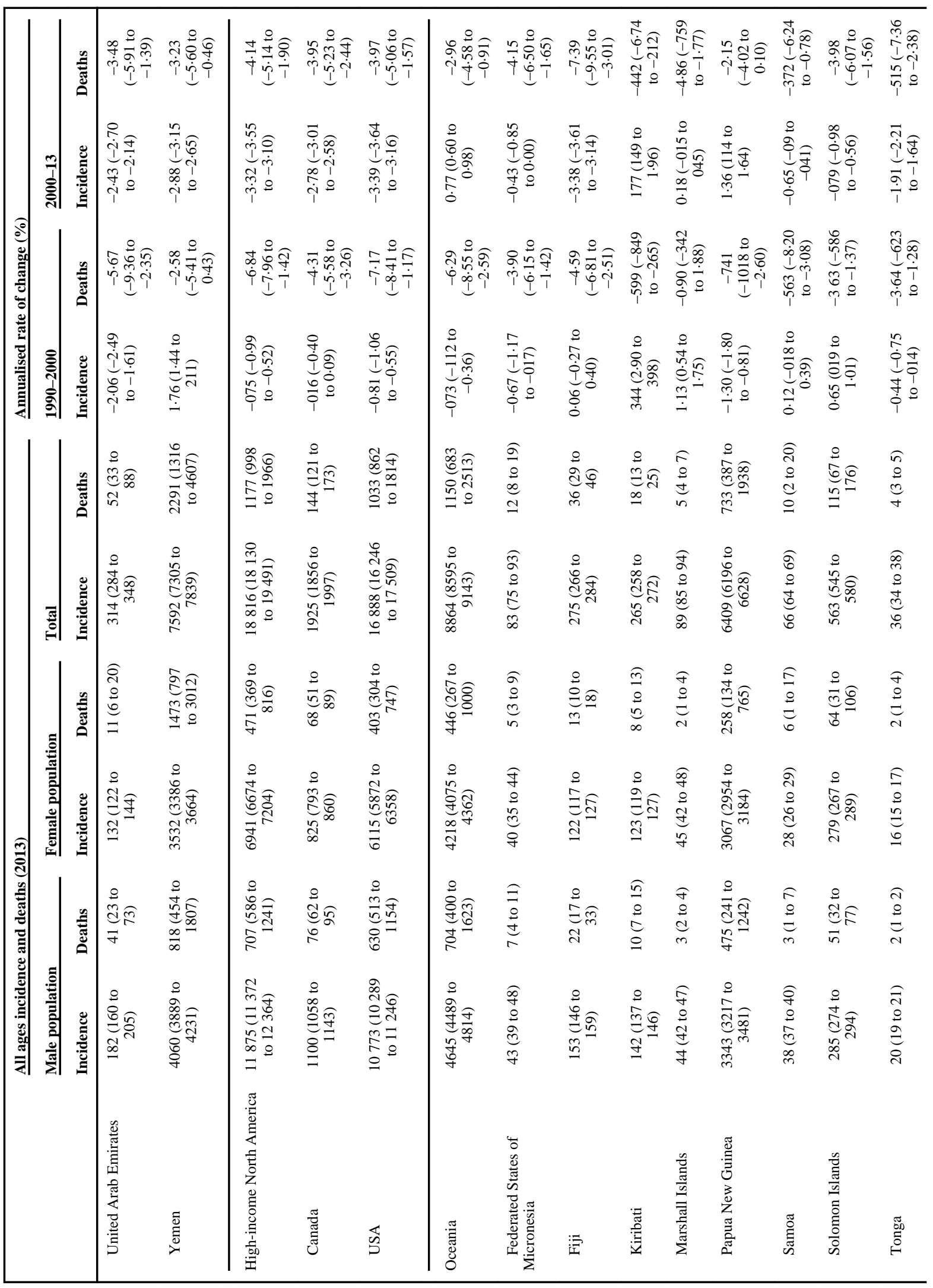




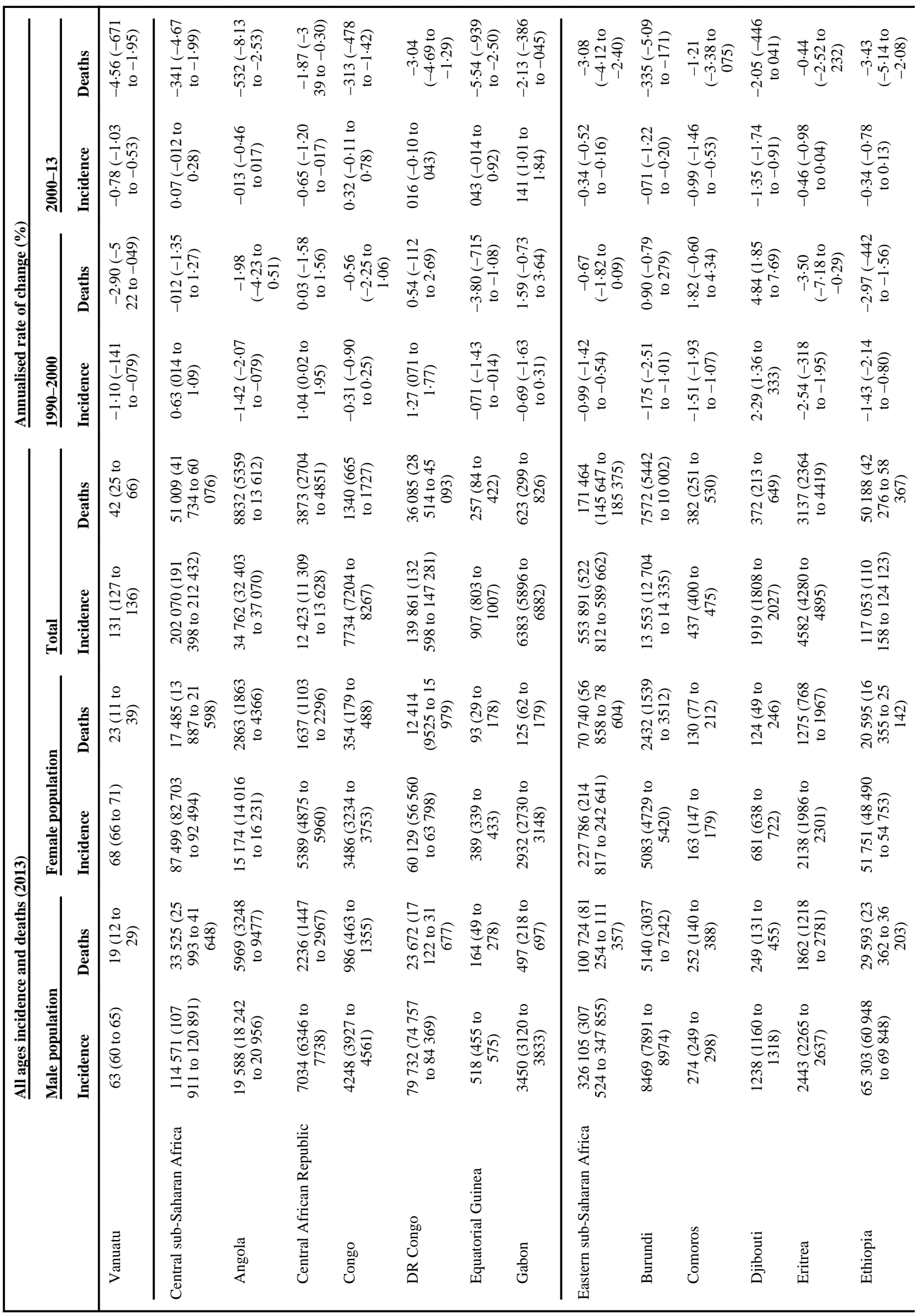




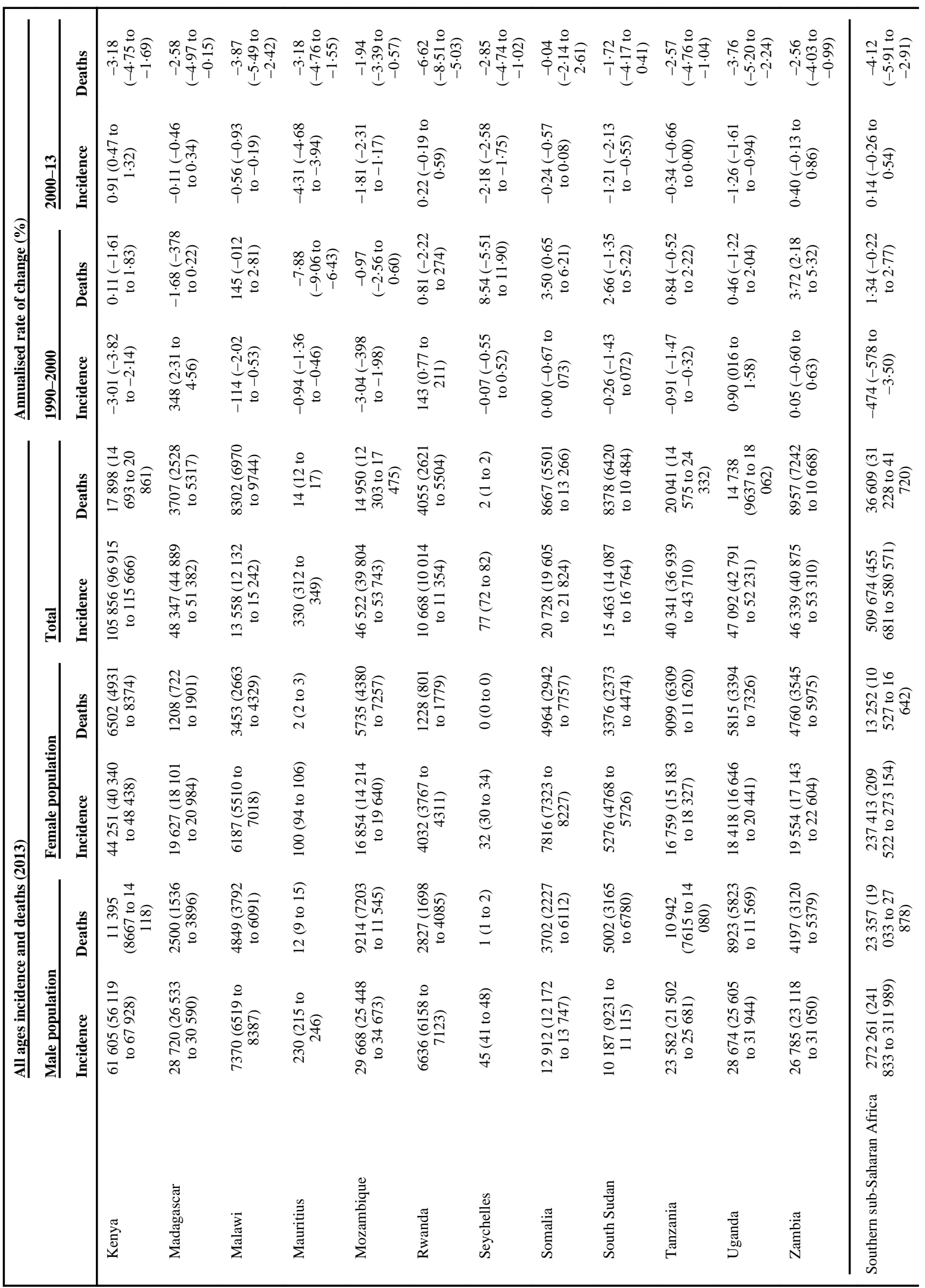




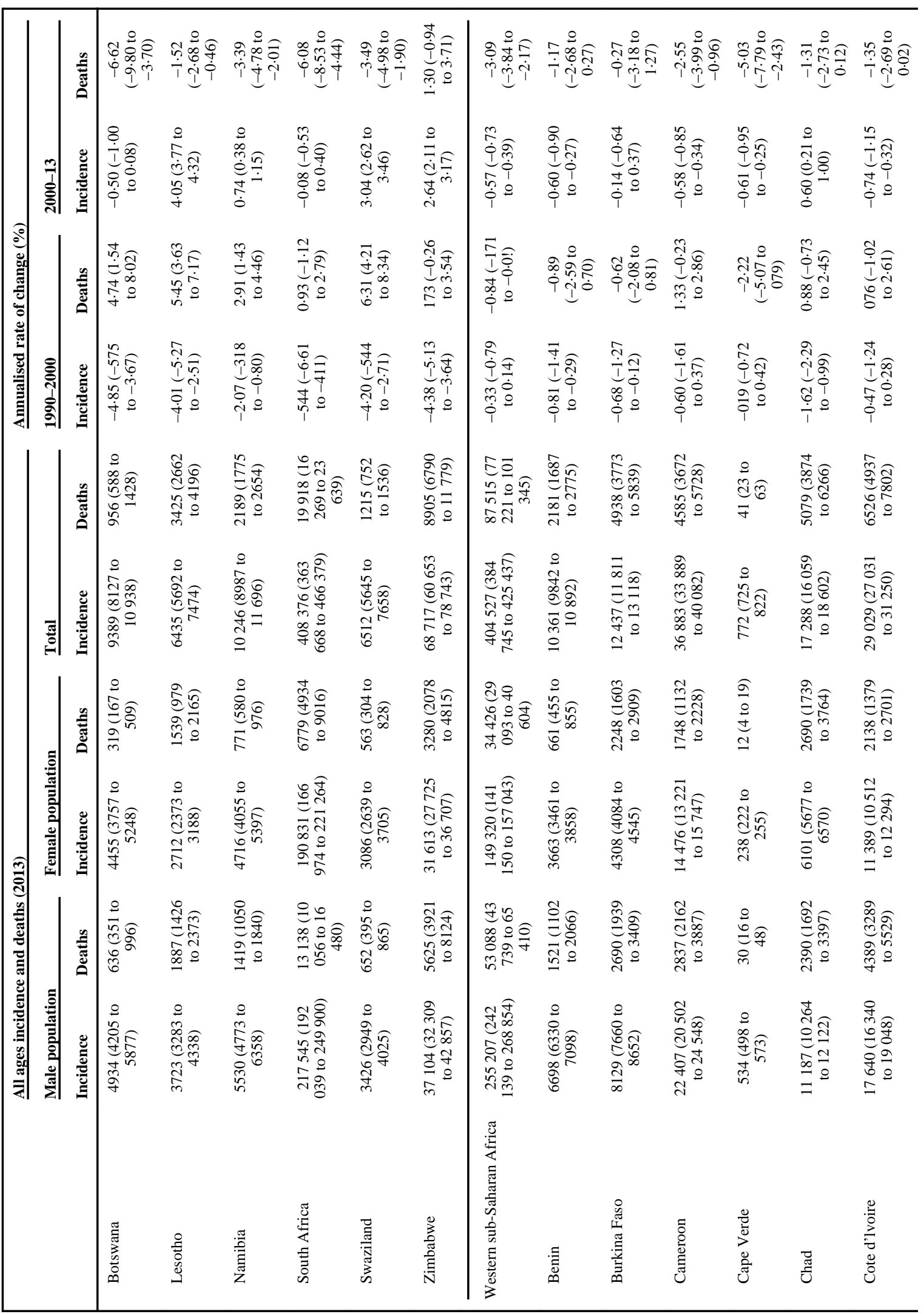




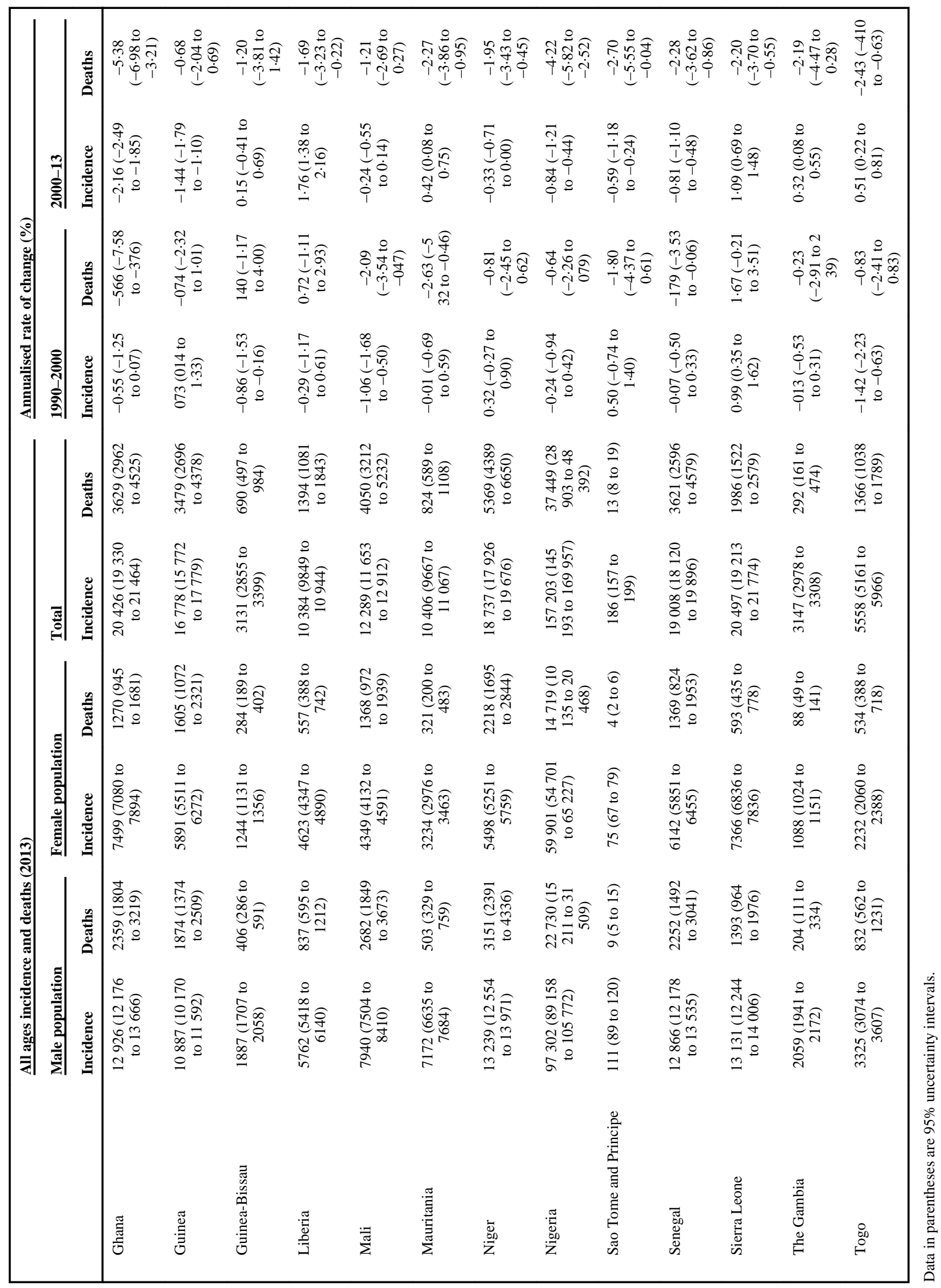


Table 6

Age-standardised malaria incidence and mortality rates and annualised rates of change for both sexes for 16 Global Burden of Disease regions

\begin{tabular}{|c|c|c|c|c|c|c|}
\hline & \multicolumn{2}{|c|}{$\begin{array}{l}\text { Age-standardisedrates in } 2003 \\
\text { (per } 100000 \text { population) }\end{array}$} & \multicolumn{4}{|c|}{ Annualised rate of change (\%) } \\
\hline & \multirow[t]{2}{*}{ Incidence } & \multirow[t]{2}{*}{ Mortality } & \multicolumn{2}{|l|}{ 1990-2000 } & \multicolumn{2}{|l|}{$\underline{2000-13}$} \\
\hline & & & Incidence & Mortality & Incidence & Mortality \\
\hline Worldwide & $\begin{array}{l}2360 \cdot 42 \\
(1373 \cdot 81 \text { to } \\
4051 \cdot 98)\end{array}$ & $\begin{array}{l}11.78(9.69 \text { to } \\
14.21)\end{array}$ & $\begin{array}{l}0 \cdot 26(-1 \cdot 02 \text { to } \\
1 \cdot 12)\end{array}$ & $\begin{array}{l}1.96(0.84 \text { to } \\
3.06)\end{array}$ & $\begin{array}{l}-3 \cdot 27(-4 \cdot 99 \text { to } \\
-1 \cdot 18)\end{array}$ & $\begin{array}{l}-3 \cdot 11(-4 \cdot 72 \text { to } \\
-1 \cdot 60)\end{array}$ \\
\hline High-income Asia Pacific & $\begin{array}{l}0.40(0.34 \text { to } \\
0 \cdot 47)\end{array}$ & $\begin{array}{l}0.00(0.00 \text { to } \\
0 \cdot 00)\end{array}$ & $\begin{array}{l}-8.48(-9.93 \text { to } \\
-7.09)\end{array}$ & $\begin{array}{l}-18 \cdot 55(-19 \cdot 35 \\
\text { to }-17 \cdot 73)\end{array}$ & $\begin{array}{l}-1 \cdot 67(-2 \cdot 28 \text { to } \\
-1 \cdot 18)\end{array}$ & $\begin{array}{l}-16 \cdot 79(-17 \cdot 75 \\
\text { to }-15 \cdot 86)\end{array}$ \\
\hline Central Asia & $\begin{array}{l}0 \cdot 19(0 \cdot 16 \text { to } \\
0 \cdot 21)\end{array}$ & $\begin{array}{l}0.02(0.01 \text { to } \\
0.03)\end{array}$ & $\begin{array}{l}16 \cdot 77(15 \cdot 48 \text { to } \\
18 \cdot 21)\end{array}$ & $\begin{array}{l}-4 \cdot 84(-9 \cdot 03 \text { to } \\
-0 \cdot 29)\end{array}$ & $\begin{array}{l}-38 \cdot 41(-39.92 \\
\text { to }-37 \cdot 09)\end{array}$ & $\begin{array}{l}-7 \cdot 32(-10 \cdot 76 \\
\text { to }-3 \cdot 15)\end{array}$ \\
\hline East Asia & $\begin{array}{l}0 \cdot 23(0 \cdot 20 \text { to } \\
0 \cdot 25)\end{array}$ & $\begin{array}{l}0.01(0.01 \text { to } \\
0 \cdot 01)\end{array}$ & $\begin{array}{l}-0 \cdot 60(-1.44 \text { to } \\
0 \cdot 36)\end{array}$ & $\begin{array}{l}-7 \cdot 67(-9 \cdot 34 \text { to } \\
-6 \cdot 02)\end{array}$ & $\begin{array}{l}-19 \cdot 91(-20 \cdot 96 \\
\text { to }-18 \cdot 98)\end{array}$ & $\begin{array}{l}-10 \cdot 95(-13 \cdot 09 \\
\text { to }-8 \cdot 36)\end{array}$ \\
\hline South Asia & $\begin{array}{l}4428 \cdot 64 \\
(1639 \cdot 86 \text { to } 10 \\
388 \cdot 82)\end{array}$ & $\begin{array}{l}9.08(7.00 \text { to } \\
11.73)\end{array}$ & $\begin{array}{l}-2 \cdot 00(-3.78 \text { to } \\
-0 \cdot 81)\end{array}$ & $\begin{array}{l}-2 \cdot 65(-4 \cdot 27 \text { to } \\
-0 \cdot 91)\end{array}$ & $\begin{array}{l}-3 \cdot 33(-4 \cdot 67 \text { to } \\
-1 \cdot 81)\end{array}$ & $\begin{array}{l}-3 \cdot 39(-5 \cdot 45 \text { to } \\
-1 \cdot 31)\end{array}$ \\
\hline Southeast Asia & $\begin{array}{l}1231 \cdot 49 \\
(556 \cdot 12 \text { to } \\
2635 \cdot 04)\end{array}$ & $\begin{array}{l}2 \cdot 28(1.68 \text { to } \\
3 \cdot 15)\end{array}$ & $\begin{array}{l}-3 \cdot 89(-5 \cdot 56 \text { to } \\
-2 \cdot 16)\end{array}$ & $\begin{array}{l}-4 \cdot 54(-6 \cdot 53 \text { to } \\
-2 \cdot 65)\end{array}$ & $\begin{array}{l}-5 \cdot 88(-8 \cdot 59 \text { to } \\
-3 \cdot 01)\end{array}$ & $\begin{array}{l}-6 \cdot 89(-9 \cdot 01 \text { to } \\
-4 \cdot 25)\end{array}$ \\
\hline Caribbean & $\begin{array}{l}245 \cdot 54(227 \cdot 17 \\
\text { to } 263 \cdot 02)\end{array}$ & $\begin{array}{l}0.91(0.51 \text { to } \\
1.47)\end{array}$ & $\begin{array}{l}-3.35(-3.63 \text { to } \\
-3.05)\end{array}$ & $\begin{array}{l}-4 \cdot 52(-6.90 \text { to } \\
-1.78)\end{array}$ & $\begin{array}{l}-4 \cdot 08(-4.64 \text { to } \\
-3.53)\end{array}$ & $\begin{array}{l}-7 \cdot 48(-11.51 \\
\text { to }-3 \cdot 38)\end{array}$ \\
\hline Andean Latin America & $\begin{array}{l}49 \cdot 36(42 \cdot 60 \text { to } \\
55 \cdot 80)\end{array}$ & $\begin{array}{l}0.04(0.03 \text { to } \\
0.05)\end{array}$ & $\begin{array}{l}-2.53(-3.04 \text { to } \\
-2 \cdot 04)\end{array}$ & $\begin{array}{l}-5 \cdot 70(-7 \cdot 28 \text { to } \\
-4 \cdot 09)\end{array}$ & $\begin{array}{l}-2.79(-3.64 \text { to } \\
-2.05)\end{array}$ & $\begin{array}{l}-13 \cdot 80(-15 \cdot 92 \\
\text { to }-11 \cdot 45)\end{array}$ \\
\hline Central Latin America & $\begin{array}{l}46 \cdot 12(40 \cdot 32 \text { to } \\
51 \cdot 63)\end{array}$ & $\begin{array}{l}0.05(0.04 \text { to } \\
0.07)\end{array}$ & $\begin{array}{l}-2 \cdot 29(-2.75 \text { to } \\
-1 \cdot 85)\end{array}$ & $\begin{array}{l}-5 \cdot 58(-7 \cdot 13 \text { to } \\
-4 \cdot 18)\end{array}$ & $\begin{array}{l}-2 \cdot 10(-2 \cdot 76 \text { to } \\
-1 \cdot 51)\end{array}$ & $\begin{array}{l}-10 \cdot 06(-12 \cdot 13 \\
\text { to }-7 \cdot 34)\end{array}$ \\
\hline Southern Latin America & $\begin{array}{l}0.95(0.81 \text { to } \\
1.08)\end{array}$ & $\begin{array}{l}0.00(0.00 \text { to } \\
0.00)\end{array}$ & $\begin{array}{l}-9.52(-10 \cdot 71 \\
\text { to }-8 \cdot 32)\end{array}$ & $\begin{array}{l}-16 \cdot 26(-17 \cdot 13 \\
\text { to }-15 \cdot 40)\end{array}$ & $\begin{array}{l}-3 \cdot 21(-4.06 \text { to } \\
-2 \cdot 48)\end{array}$ & $\begin{array}{l}-16 \cdot 29(-17 \cdot 17 \\
\text { to }-15 \cdot 43)\end{array}$ \\
\hline Tropical Latin America & $\begin{array}{l}62 \cdot 80(53 \cdot 39 \text { to } \\
71 \cdot 76)\end{array}$ & $\begin{array}{l}0.03(0.02 \text { to } \\
0.05)\end{array}$ & $\begin{array}{l}-6 \cdot 49(-7 \cdot 87 \text { to } \\
-5 \cdot 20)\end{array}$ & $\begin{array}{l}-17 \cdot 58(-19 \cdot 80 \\
\text { to }-15 \cdot 41)\end{array}$ & $\begin{array}{l}-0 \cdot 11(-0 \cdot 50 \text { to } \\
0 \cdot 21)\end{array}$ & $\begin{array}{l}-9 \cdot 20(-12 \cdot 22 \\
\text { to }-5 \cdot 86)\end{array}$ \\
\hline North Africa and Middle East & $\begin{array}{l}396 \cdot 71(122 \cdot 46 \\
\text { to } 1028 \cdot 80)\end{array}$ & $\begin{array}{l}1.97(1.07 \text { to } \\
3.48)\end{array}$ & $\begin{array}{l}2 \cdot 14(1 \cdot 31 \text { to } \\
3 \cdot 18)\end{array}$ & $\begin{array}{l}3.88(0.73 \text { to } \\
7.01)\end{array}$ & $\begin{array}{l}-5 \cdot 74(-9 \cdot 08 \text { to } \\
-2 \cdot 40)\end{array}$ & $\begin{array}{l}-7 \cdot 07(-10 \cdot 62 \\
\text { to }-3 \cdot 04)\end{array}$ \\
\hline Oceania & $\begin{array}{l}10452 \cdot 65 \\
(3908 \cdot 47 \text { to } 25 \\
253 \cdot 80)\end{array}$ & $\begin{array}{l}20 \cdot 54(11.95 \text { to } \\
33 \cdot 16)\end{array}$ & $\begin{array}{l}-0 \cdot 02(-0.25 \text { to } \\
0 \cdot 19)\end{array}$ & $\begin{array}{l}-0 \cdot 30(-3 \cdot 70 \text { to } \\
2 \cdot 75)\end{array}$ & $\begin{array}{l}-2 \cdot 14(-3 \cdot 14 \text { to } \\
-1 \cdot 05)\end{array}$ & $\begin{array}{l}-2.61(-5.49 \text { to } \\
0.65)\end{array}$ \\
\hline Central sub-Saharan Africa & $\begin{array}{l}6628 \cdot 47 \\
(3171 \cdot 03 \text { to } 13 \\
240 \cdot 91)\end{array}$ & $\begin{array}{l}43 \cdot 15(28 \cdot 36 \text { to } \\
63 \cdot 55)\end{array}$ & $\begin{array}{l}-2.74(-4.45 \text { to } \\
-1.39)\end{array}$ & $\begin{array}{l}-1.59(-4.08 \text { to } \\
1.08)\end{array}$ & $\begin{array}{l}-5 \cdot 13(-7 \cdot 81 \text { to } \\
-2 \cdot 43)\end{array}$ & $\begin{array}{l}-5 \cdot 73(-9.03 \text { to } \\
-2 \cdot 28)\end{array}$ \\
\hline Eastern sub-Saharan Africa & $\begin{array}{l}6411 \cdot 66 \\
(3214 \cdot 47 \text { to } 12 \\
802 \cdot 02)\end{array}$ & $\begin{array}{l}39 \cdot 58(33 \cdot 03 \text { to } \\
49 \cdot 36)\end{array}$ & $\begin{array}{l}0 \cdot 41(-0 \cdot 29 \text { to } \\
1 \cdot 52)\end{array}$ & $\begin{array}{l}0.77(-0 \cdot 82 \text { to } \\
2 \cdot 33)\end{array}$ & $\begin{array}{l}-6 \cdot 68(-8.94 \text { to } \\
-3 \cdot 58)\end{array}$ & $\begin{array}{l}-6 \cdot 76(-8 \cdot 47 \text { to } \\
-4 \cdot 40)\end{array}$ \\
\hline
\end{tabular}




\begin{tabular}{|c|c|c|c|c|c|c|}
\hline & \multicolumn{2}{|c|}{$\begin{array}{l}\text { Age-standardisedrates in } 2003 \\
\text { (per } 100000 \text { population) }\end{array}$} & \multirow{2}{*}{\multicolumn{2}{|c|}{$\begin{array}{l}\text { Annualised rate of change (\%) } \\
\text { 1990-2000 }\end{array}$}} & \multirow{2}{*}{\multicolumn{2}{|c|}{$\underline{2000-13}$}} \\
\hline & \multirow[t]{2}{*}{ Incidence } & \multirow[t]{2}{*}{ Mortality } & & & & \\
\hline & & & Incidence & Mortality & Incidence & Mortality \\
\hline Southern sub-Saharan Africa & $\begin{array}{l}766 \cdot 01(315 \cdot 25 \\
\text { to } 1807 \cdot 70)\end{array}$ & $\begin{array}{l}4.21(3 \cdot 22 \text { to } \\
5 \cdot 76)\end{array}$ & $\begin{array}{l}2 \cdot 84(1 \cdot 28 \text { to } \\
4 \cdot 19)\end{array}$ & $\begin{array}{l}2.85(-0.40 \text { to } \\
5.45)\end{array}$ & $\begin{array}{l}-5 \cdot 63(-8.37 \text { to } \\
-2 \cdot 64)\end{array}$ & $\begin{array}{l}-7 \cdot 30(-9 \cdot 74 \text { to } \\
-4 \cdot 10)\end{array}$ \\
\hline Western sub-Saharan Africa & $\begin{array}{l}11874 \cdot 88 \\
(6907 \cdot 10 \text { to } 20 \\
684 \cdot 35)\end{array}$ & $\begin{array}{l}85.89(68.14 \text { to } \\
105.95)\end{array}$ & $\begin{array}{l}0.90(0.26 \text { to } \\
1.72)\end{array}$ & $\begin{array}{l}1.45(-0 \cdot 24 \text { to } \\
3 \cdot 18)\end{array}$ & $\begin{array}{l}-3.79(-5.33 \text { to } \\
-2 \cdot 00)\end{array}$ & $\begin{array}{l}-3 \cdot 40(-5 \cdot 15 \text { to } \\
-1 \cdot 56)\end{array}$ \\
\hline
\end{tabular}

Data in parentheses are 95\% uncertainty intervals. 


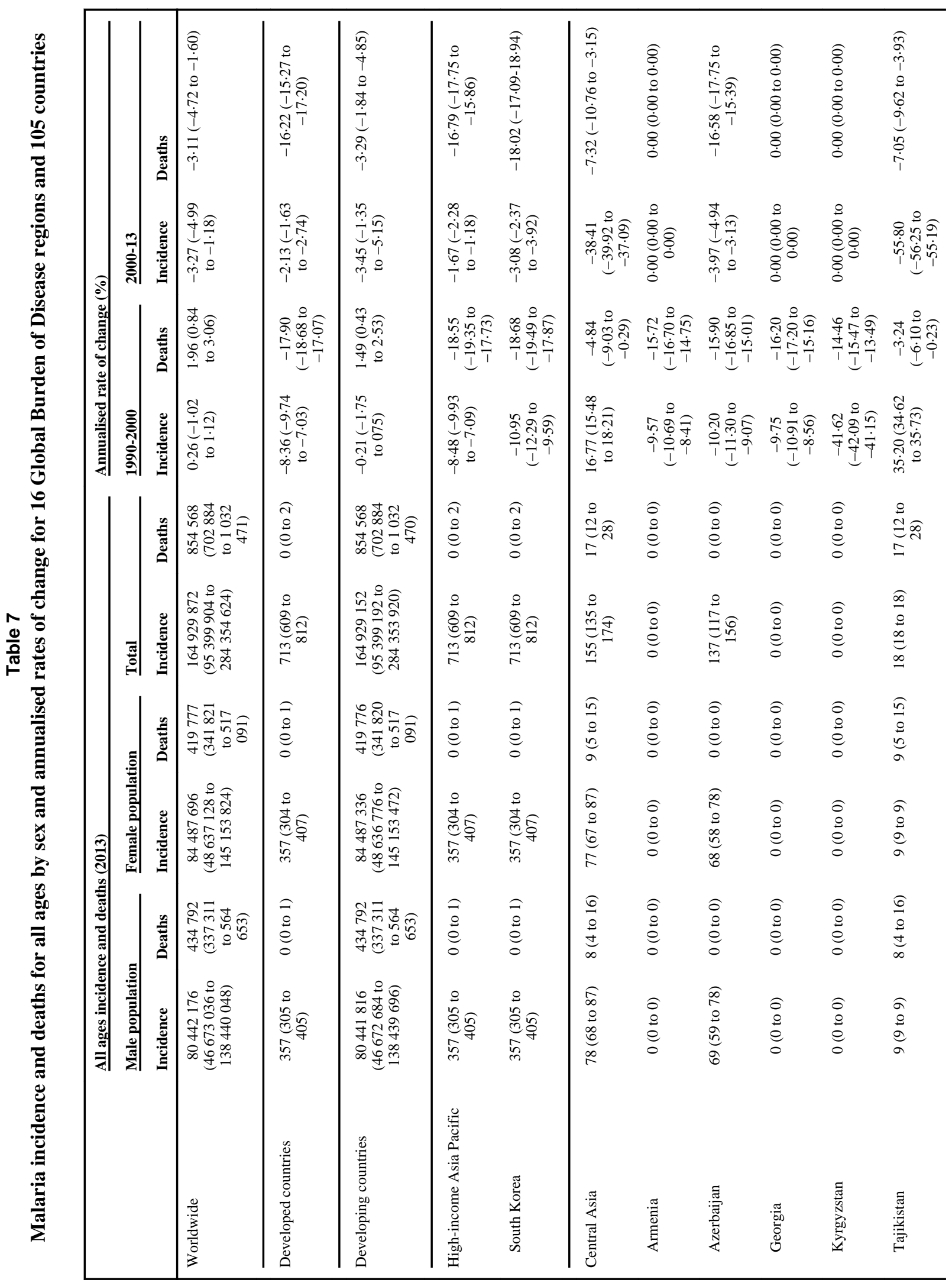




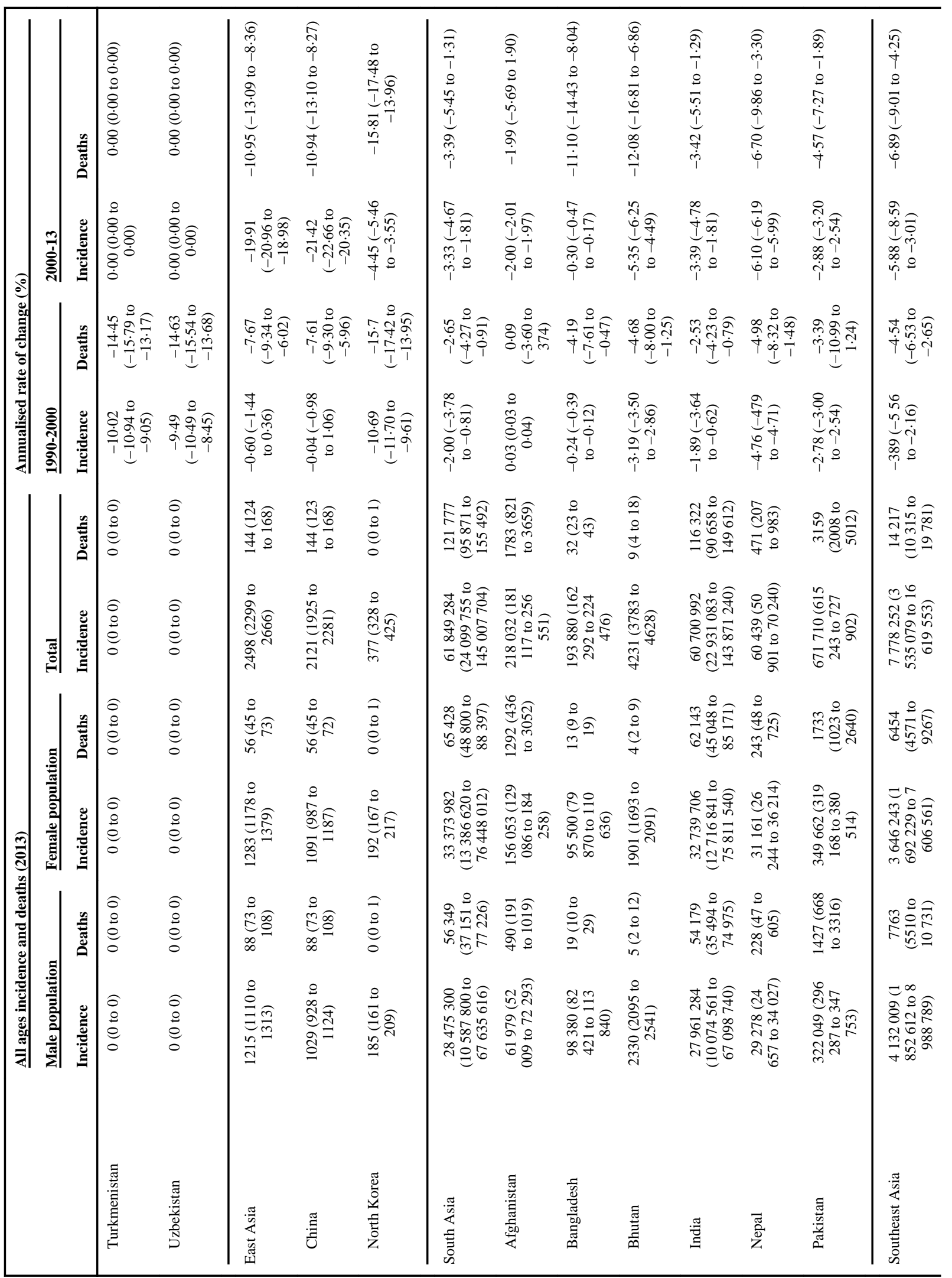




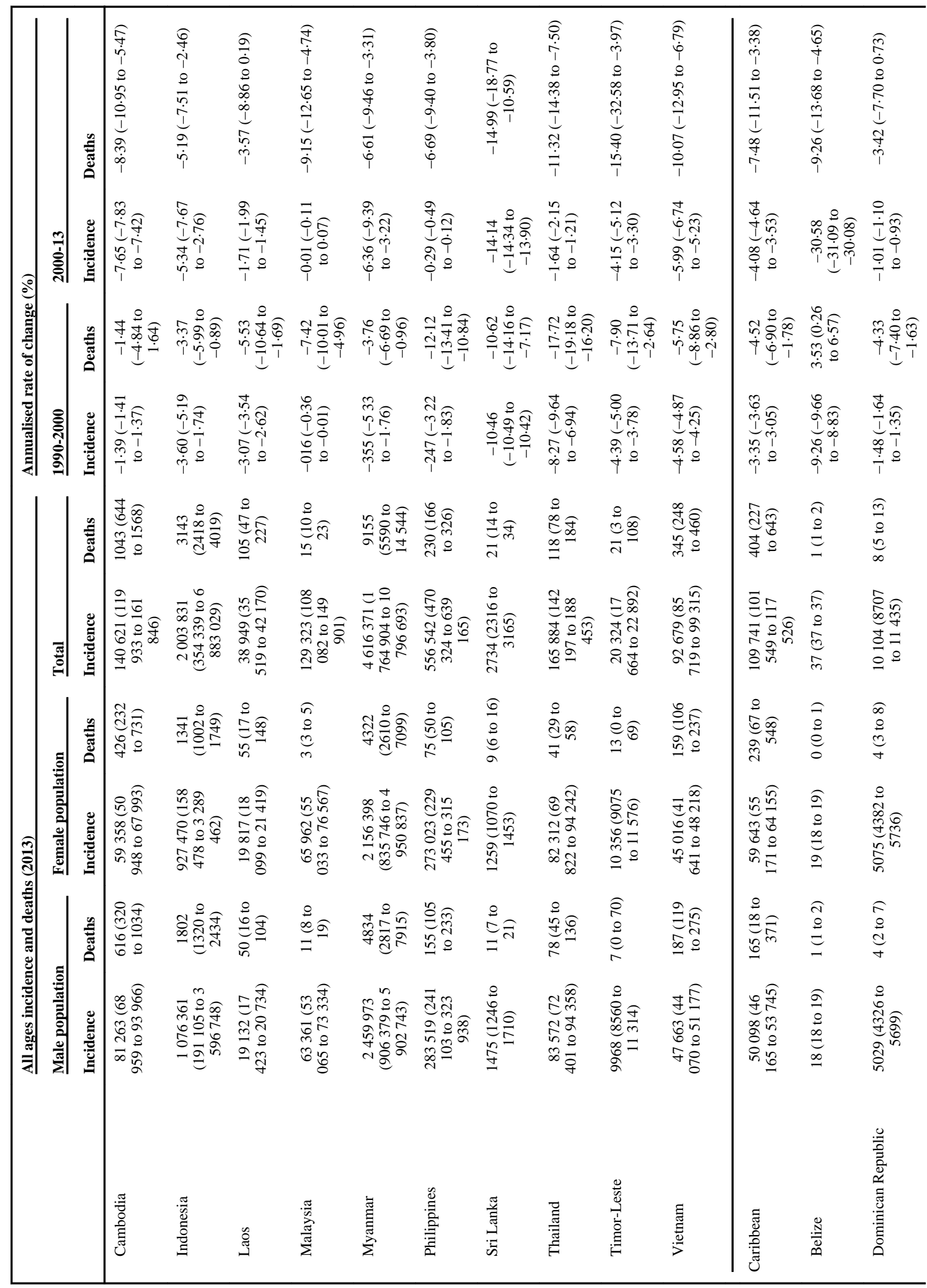




\begin{tabular}{|c|c|c|c|c|c|c|c|c|c|c|c|c|c|c|c|}
\hline & & 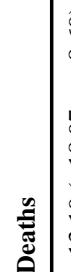 & 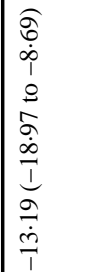 & 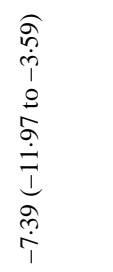 & 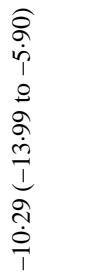 & $\begin{array}{l}\widehat{o} \\
\dot{0} \\
0 \\
0 \\
0 \\
\dot{0} \\
0 \\
0 \\
0\end{array}$ & $\begin{array}{l}\widehat{o} \\
\dot{0} \\
0 \\
0 \\
0 \\
\dot{0} \\
8 \\
0 \\
0\end{array}$ & 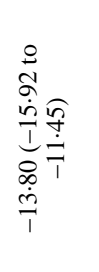 & 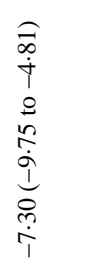 & 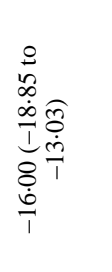 & 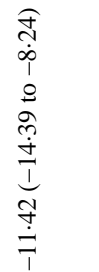 & 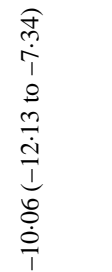 & $\begin{array}{l}\text { ले } \\
0 \\
1 \\
0 \\
0 \\
0 \\
\dot{1} \\
\dot{I} \\
\underline{0} \\
0 \\
0 \\
0 \\
1\end{array}$ & 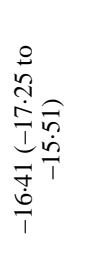 & 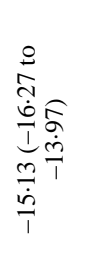 \\
\hline a & 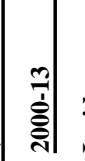 & 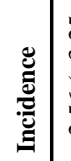 & 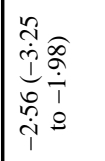 & 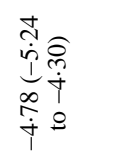 & 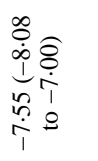 & 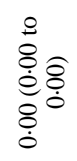 & $\begin{array}{l}\stackrel{8}{8} \\
8 \\
\dot{8} \\
\dot{8} \\
8 \\
0 \\
0\end{array}$ & 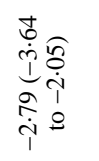 & 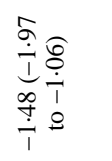 & 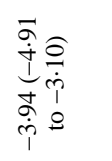 & 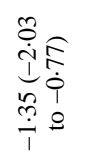 & 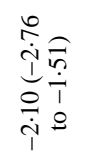 & 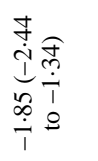 & 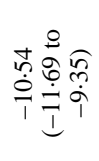 & 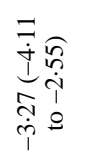 \\
\hline 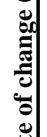 & 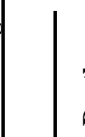 & 鸪 & 它 & ํㅜㄹ & 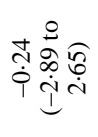 & 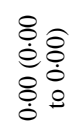 & $\begin{array}{l}8 \\
\dot{0} \\
\dot{0} \\
8 \\
0 \\
0 \\
0\end{array}$ & 突离 & 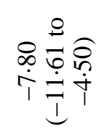 & 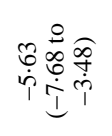 & 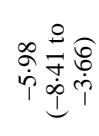 & î & 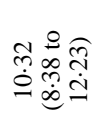 & 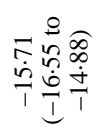 & 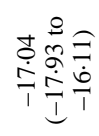 \\
\hline 矛 & : & 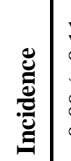 & 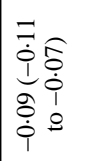 & 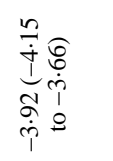 & 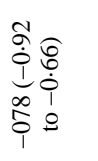 & $\begin{array}{l}0 \\
\stackrel{8}{0} \\
\dot{0} \\
\stackrel{0}{0} \\
\dot{0} \\
\dot{0}\end{array}$ & 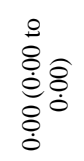 & 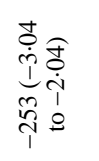 & 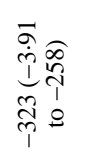 & 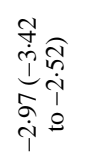 & 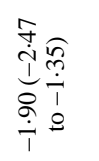 & 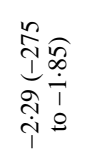 & 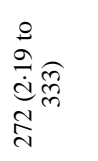 & 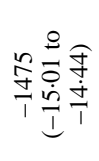 & 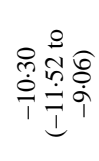 \\
\hline & & . & 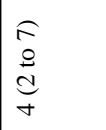 & 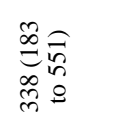 & 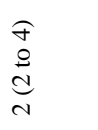 & $\begin{array}{l}0 \\
0 \\
0 \\
0 \\
0\end{array}$ & $\begin{array}{l}0 \\
0 \\
0 \\
0 \\
0\end{array}$ & $\underbrace{\infty}_{\substack{\infty \\
\infty}} \widehat{\hat{\lambda}}$ & $\begin{array}{l}\hat{\sigma} \\
\stackrel{2}{d} \\
\stackrel{d}{N}\end{array}$ & 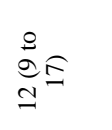 & $\begin{array}{l}\widehat{a} \\
0 \\
0 \\
0 \\
\infty\end{array}$ & 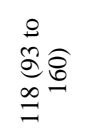 & 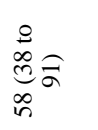 & $\begin{array}{l}0 \\
0 \\
0 \\
0 \\
0\end{array}$ & $\begin{array}{l}0 \\
0 \\
0 \\
0 \\
0\end{array}$ \\
\hline & ? & 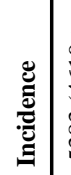 & 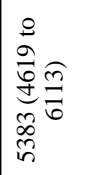 & 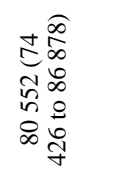 & 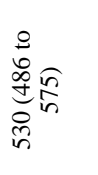 & $\begin{array}{l}\hat{2} \\
0 \\
0 \\
0 \\
m\end{array}$ & $\begin{array}{l}\hat{m} \\
0 \\
0 \\
m \\
m\end{array}$ & 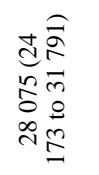 & 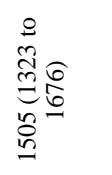 & 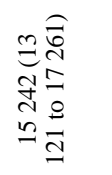 & 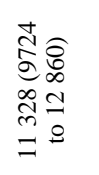 & 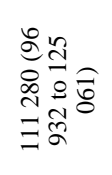 & 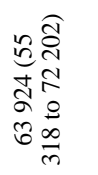 & $\begin{array}{l}\hat{a} \\
\stackrel{0}{\infty} \\
\alpha \\
\alpha\end{array}$ & $\begin{array}{l}\sigma \\
\delta \\
0 \\
o \\
\delta \\
\sigma\end{array}$ \\
\hline & & : & $\begin{array}{l}\widehat{O} \\
\stackrel{\Xi}{\Xi} \\
=\end{array}$ & 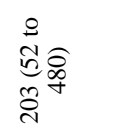 & $\stackrel{0}{\stackrel{0}{0}}$ & $\begin{array}{l}0 \\
\vdots \\
0 \\
0 \\
0\end{array}$ & $\begin{array}{l}0 \\
0 \\
0 \\
0 \\
0\end{array}$ & $\stackrel{\stackrel{\infty}{\infty}}{\varrho} \Im$ & $\begin{array}{l}\hat{\imath} \\
\stackrel{\Xi}{\Xi}\end{array}$ & 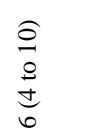 & 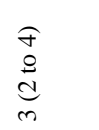 & 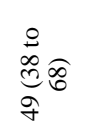 & $\underset{\substack{i \\
d}}{\stackrel{j}{m}}$ & $\begin{array}{l}0 \\
0 \\
0 \\
0 \\
0\end{array}$ & $\begin{array}{l}0 \\
0 \\
o \\
0 \\
0\end{array}$ \\
\hline 웜 & 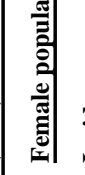 & 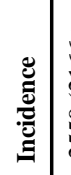 & 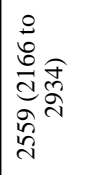 & 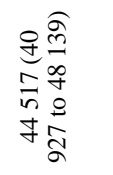 & 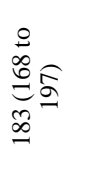 & $\begin{array}{l}0 \\
0 \\
0 \\
0 \\
0\end{array}$ & $\begin{array}{l}0 \\
0 \\
o \\
0 \\
0\end{array}$ & 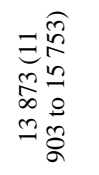 & 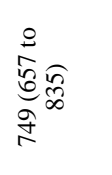 & 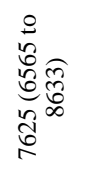 & 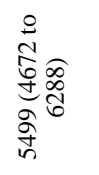 & 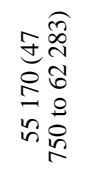 & 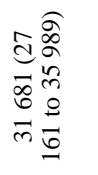 & 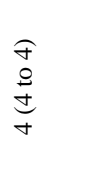 & 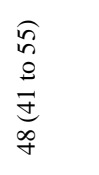 \\
\hline 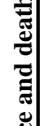 & & 号 & $\begin{array}{l}n \\
\vdots \\
\stackrel{5}{=} \\
m\end{array}$ & 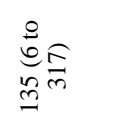 & 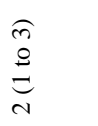 & $\begin{array}{l}0 \\
0 \\
0 \\
0 \\
0\end{array}$ & $\begin{array}{l}0 \\
0 \\
o \\
0 \\
0\end{array}$ & $\underbrace{\stackrel{g}{g}}_{g}$ & $\begin{array}{l}\widehat{\hat{\jmath}} \\
\stackrel{\Xi}{\Xi} \\
\Xi\end{array}$ & 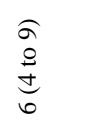 & \begin{tabular}{l}
0 \\
0 \\
0 \\
\multirow{0}{*}{} \\
$n$
\end{tabular} & 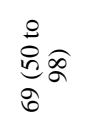 & 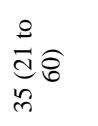 & $\begin{array}{l}0 \\
0 \\
0 \\
0 \\
0\end{array}$ & 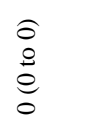 \\
\hline 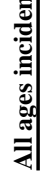 & 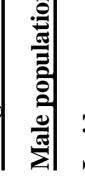 & : & 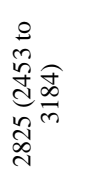 & 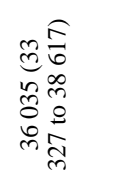 & 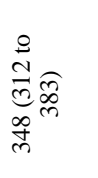 & $\begin{array}{l}0 \\
0 \\
0 \\
0 \\
0\end{array}$ & $\begin{array}{l}0 \\
0 \\
0 \\
0 \\
0 \\
0\end{array}$ & 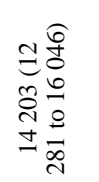 & 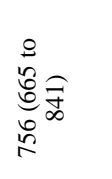 & 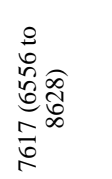 & 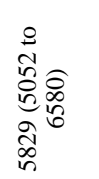 & 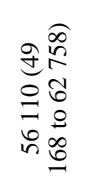 & 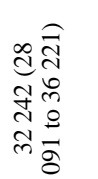 & $\begin{array}{l}n \\
0 \\
0 \\
\vdots \\
n \\
n\end{array}$ & 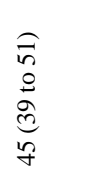 \\
\hline & & & 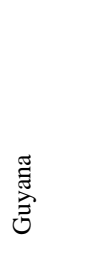 & 潯 & 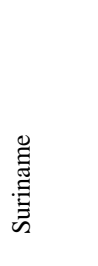 & 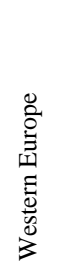 & 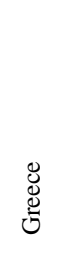 & 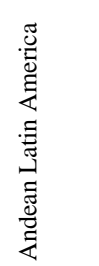 & 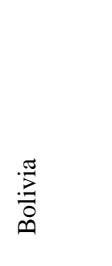 & 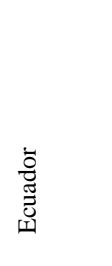 & 离 & 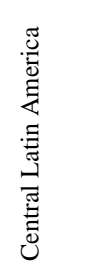 & $\begin{array}{l}\frac{\pi}{0} \\
\frac{0}{0} \\
\frac{0}{0}\end{array}$ & 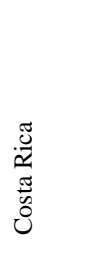 & 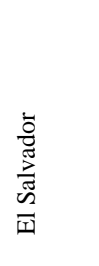 \\
\hline
\end{tabular}




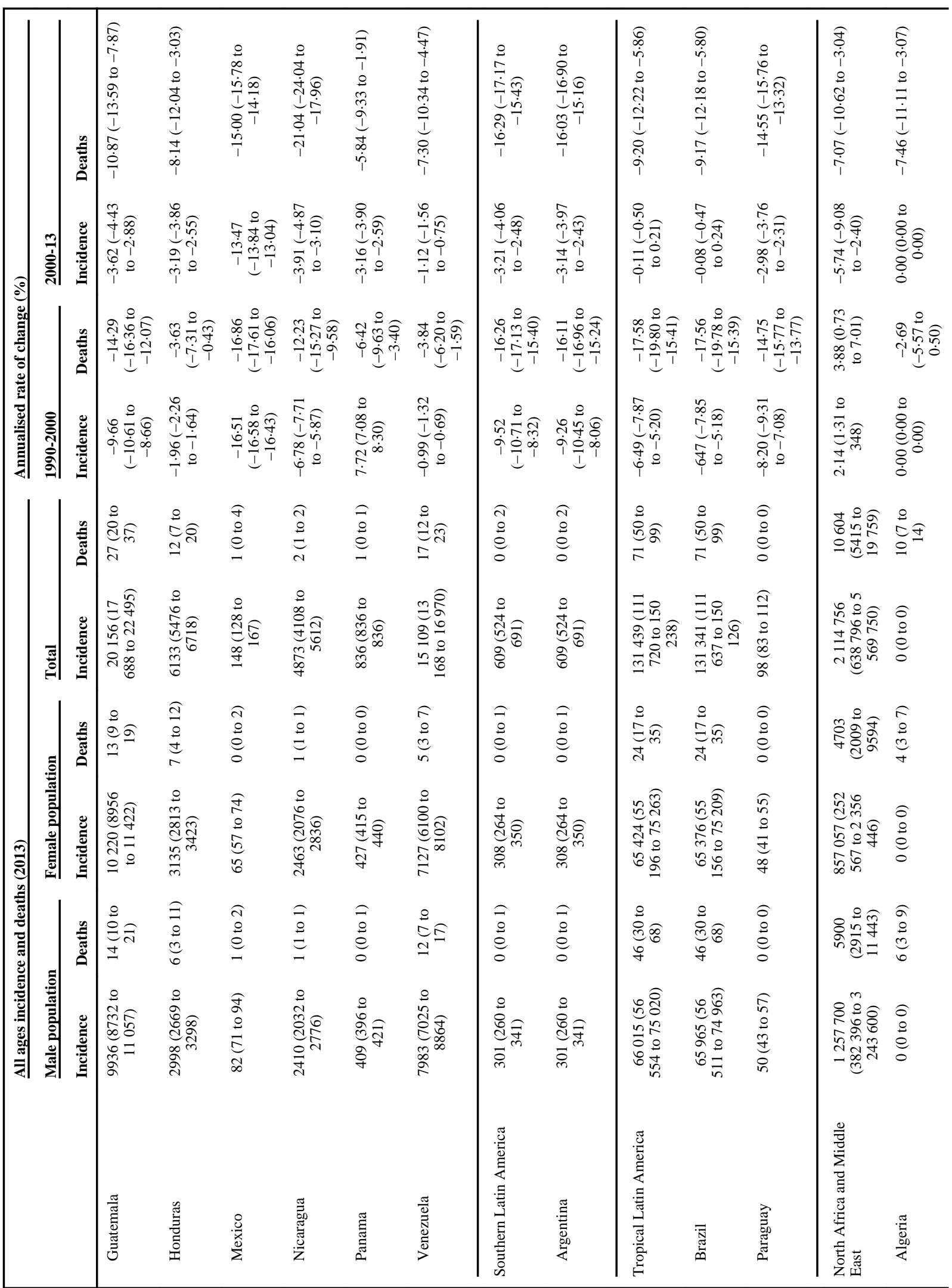




\begin{tabular}{|c|c|c|c|c|c|c|c|c|c|c|c|c|c|c|c|}
\hline & & $\mid$ & 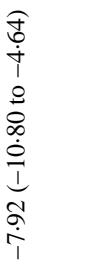 & $\begin{array}{l}\widehat{o} \\
\dot{0} \\
0 \\
0 \\
0 \\
\dot{0} \\
0 \\
0 \\
0\end{array}$ & $\begin{array}{l}\widehat{8} \\
\dot{0} \\
0 \\
8 \\
8 \\
\dot{0} \\
8 \\
0 \\
0\end{array}$ & $\begin{array}{l}\widehat{o} \\
\dot{0} \\
0 \\
0 \\
0 \\
0 \\
\dot{0} \\
8 \\
0 \\
0\end{array}$ & 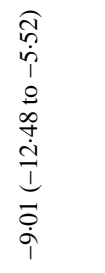 & 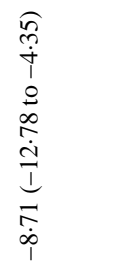 & $\begin{array}{l}\widehat{o} \\
\dot{0} \\
0 \\
0 \\
0 \\
\dot{0} \\
8 \\
0 \\
0\end{array}$ & 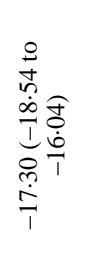 & 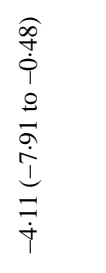 & $\begin{array}{l}\sqrt{0} \\
\dot{0} \\
0 \\
0 \\
\sigma \\
\dot{f} \\
i \\
i \\
\dot{d} \\
i \\
i\end{array}$ & 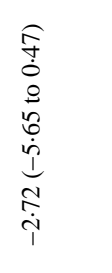 & 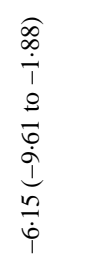 & 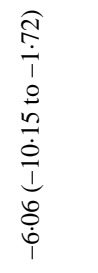 \\
\hline$\therefore$ & 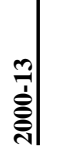 & 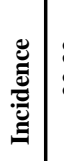 & 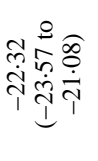 & 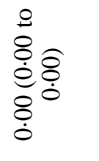 & 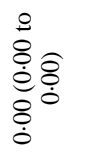 & $\begin{array}{l}8 \\
8 \\
0 \\
0 \\
0 \\
8 \\
0 \\
0\end{array}$ & 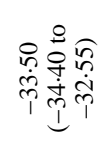 & 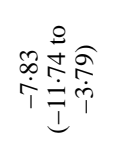 & $\begin{array}{l}8 \\
8 \\
8 \\
\dot{0} \\
8_{0}^{\circ} \\
\dot{0}\end{array}$ & 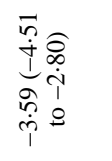 & 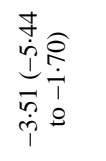 & 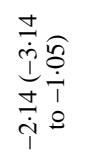 & 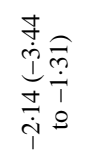 & 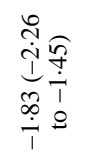 & 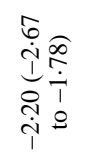 \\
\hline 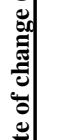 & & 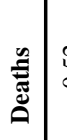 & 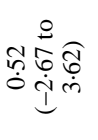 & 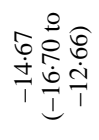 & 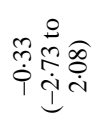 & ஸे & 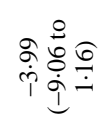 & 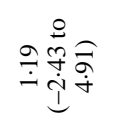 & 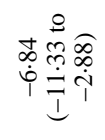 & 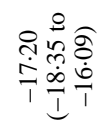 & 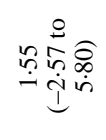 & 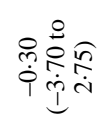 & 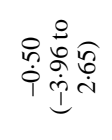 & 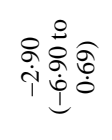 & 突窟完 \\
\hline 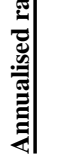 & : & 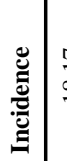 & 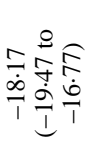 & 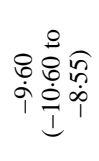 & 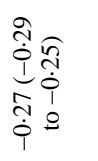 & 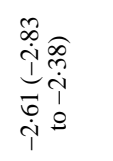 & 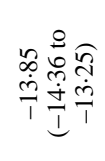 & 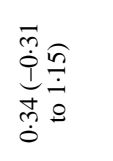 & 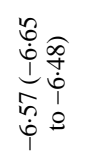 & 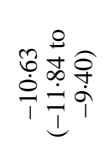 & 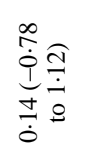 & 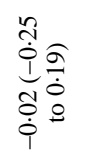 & 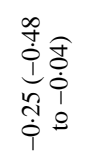 & 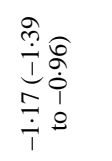 & 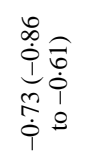 \\
\hline & & $\stackrel{\underline{g}}{\vec{z}}$ & $\stackrel{8}{\infty} \underset{\varrho}{\infty}$ & $\begin{array}{l}0 \\
0 \\
0 \\
0 \\
0 \\
0\end{array}$ & $\begin{array}{l}0 \\
0 \\
0 \\
0 \\
0 \\
0\end{array}$ & $\begin{array}{l}0 \\
0 \\
0 \\
0 \\
0 \\
0\end{array}$ & $\stackrel{\circ}{a} \infty$ & 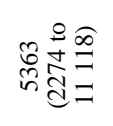 & $\begin{array}{l}0 \\
0 \\
0 \\
0 \\
0\end{array}$ & $\begin{array}{l}\hat{\imath} \\
\stackrel{0}{o} \\
\stackrel{e}{-}\end{array}$ & 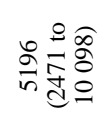 & 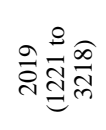 & 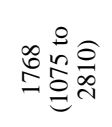 & 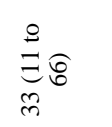 & 总 \\
\hline & . & 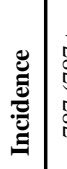 & 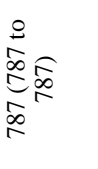 & $\begin{array}{l}\hat{\sigma} \\
0 \\
\stackrel{0}{0} \\
0\end{array}$ & $\begin{array}{l}0 \\
0 \\
0 \\
0 \\
0\end{array}$ & $\begin{array}{l}0 \\
0 \\
0 \\
0 \\
0 \\
0\end{array}$ & 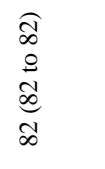 & 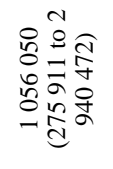 & $\begin{array}{l}0 \\
0 \\
0 \\
0 \\
0\end{array}$ & 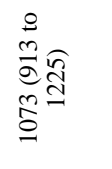 & 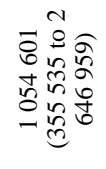 & 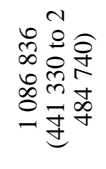 & 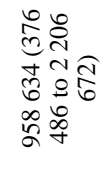 & 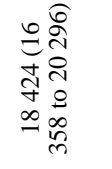 & 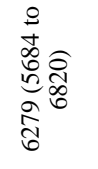 \\
\hline & & 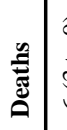 & $\begin{array}{l}\hat{2} \\
\stackrel{0}{0} \\
0 \\
0\end{array}$ & $\begin{array}{l}0 \\
0 \\
\vdots \\
0 \\
0\end{array}$ & $\begin{array}{l}0 \\
0 \\
0 \\
0 \\
0 \\
0\end{array}$ & $\begin{array}{l}0 \\
0 \\
0 \\
0 \\
0 \\
0\end{array}$ & $\begin{array}{l}\hat{n} \\
\stackrel{\leftrightarrow}{\Xi} \\
\stackrel{N}{1}\end{array}$ & 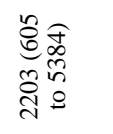 & $\begin{array}{l}0 \\
0 \\
0 \\
0 \\
0\end{array}$ & $\begin{array}{l}0 \\
\stackrel{0}{0} \\
0 \\
0 \\
0\end{array}$ & 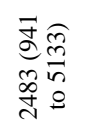 & 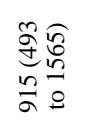 & 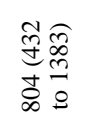 & 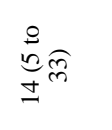 & 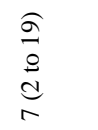 \\
\hline 司 & 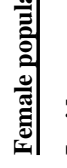 & 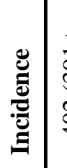 & 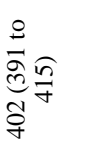 & $\begin{array}{l}0 \\
0 \\
0 \\
0 \\
0\end{array}$ & $\begin{array}{l}0 \\
0 \\
0 \\
0 \\
0 \\
0\end{array}$ & $\begin{array}{l}0 \\
0 \\
0 \\
0 \\
0\end{array}$ & 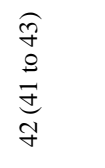 & 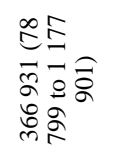 & $\begin{array}{l}0 \\
0 \\
\stackrel{0}{0} \\
0 \\
0\end{array}$ & 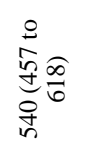 & 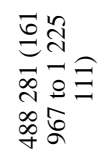 & 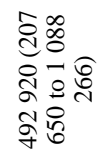 & 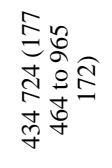 & 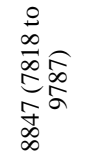 & 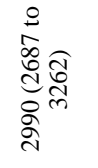 \\
\hline 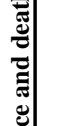 & & 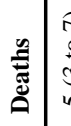 & $\begin{array}{l}\text { E } \\
0 \\
0 \\
\text { n. } \\
\text { n. }\end{array}$ & $\begin{array}{l}0 \\
\stackrel{0}{0} \\
\stackrel{0}{0} \\
0\end{array}$ & $\begin{array}{l}0 \\
0 \\
0 \\
0 \\
0 \\
0\end{array}$ & $\begin{array}{l}0 \\
0 \\
0 \\
0 \\
0\end{array}$ & $\underset{0}{\stackrel{0}{5}=}$ & 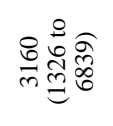 & $\begin{array}{l}0 \\
0 \\
0 \\
0 \\
0\end{array}$ & $\begin{array}{l}0 \\
0 \\
0 \\
0 \\
0\end{array}$ & 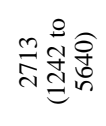 & 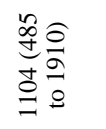 & 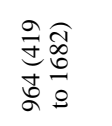 & $\stackrel{8}{\stackrel{8}{g}}$ & $\begin{array}{l}\widehat{\widehat{N}} \\
\stackrel{0}{d} \\
d \\
a\end{array}$ \\
\hline 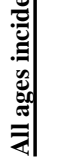 & 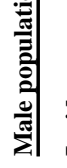 & 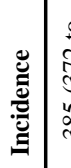 & 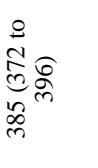 & $\begin{array}{l}0 \\
0 \\
0 \\
0 \\
0\end{array}$ & $\begin{array}{l}0 \\
0 \\
0 \\
0 \\
0 \\
0\end{array}$ & $\begin{array}{l}0 \\
\stackrel{0}{o} \\
\stackrel{0}{0} \\
0\end{array}$ & 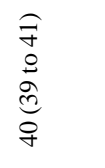 & 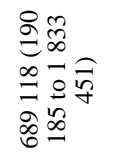 & $\begin{array}{l}0 \\
0 \\
0 \\
0 \\
0\end{array}$ & 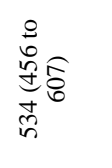 & 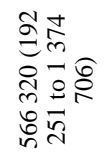 & 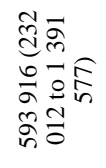 & 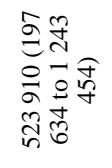 & 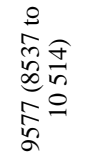 & 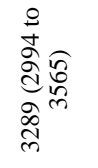 \\
\hline & & & ฐ్ & 氶 & $\begin{array}{l}\stackrel{8}{0} \\
\underbrace{\circ}_{0} \\
\sum^{\circ}\end{array}$ & 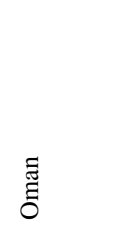 & 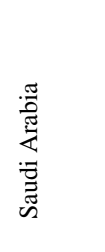 & 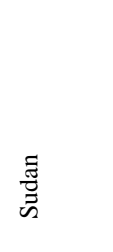 & 䍃 & 萻 & $\begin{array}{l}\overline{\bar{\Xi}} \\
\stackrel{\bar{E}}{\bar{E}}\end{array}$ & 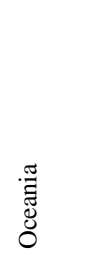 & 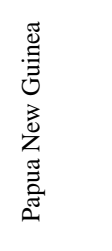 & 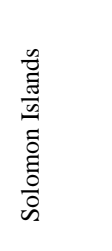 & 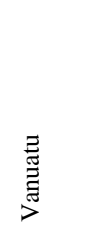 \\
\hline
\end{tabular}




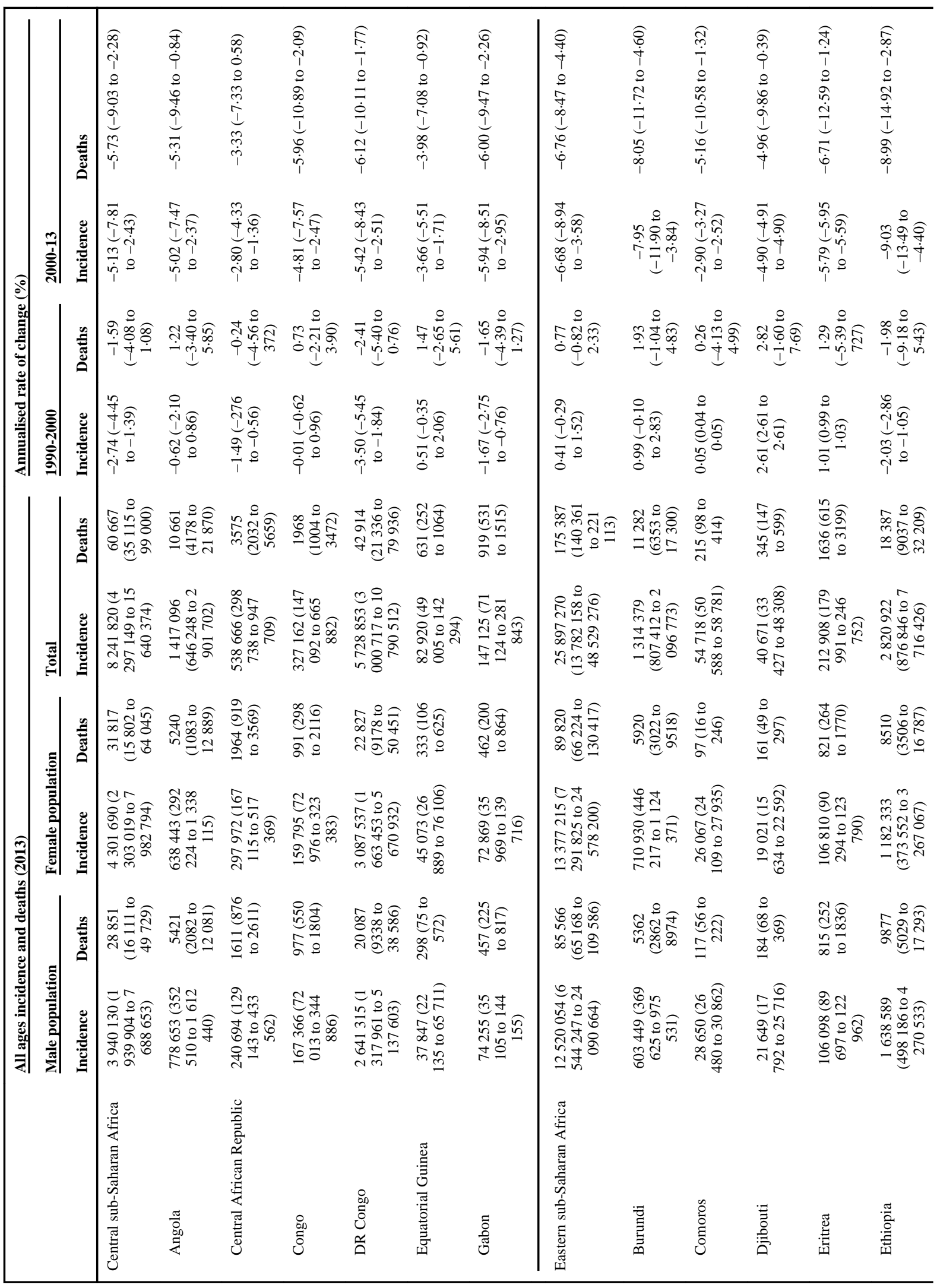




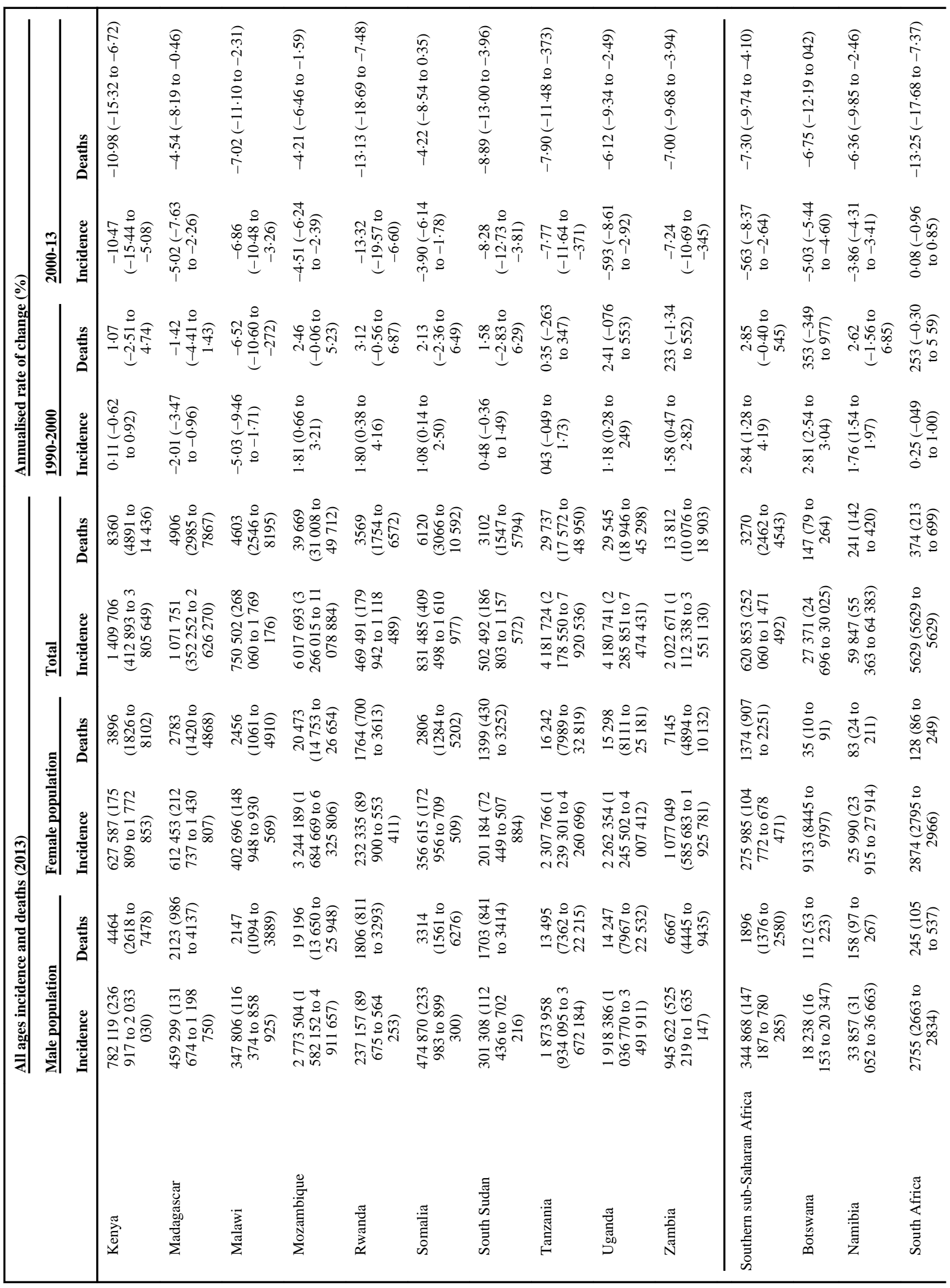




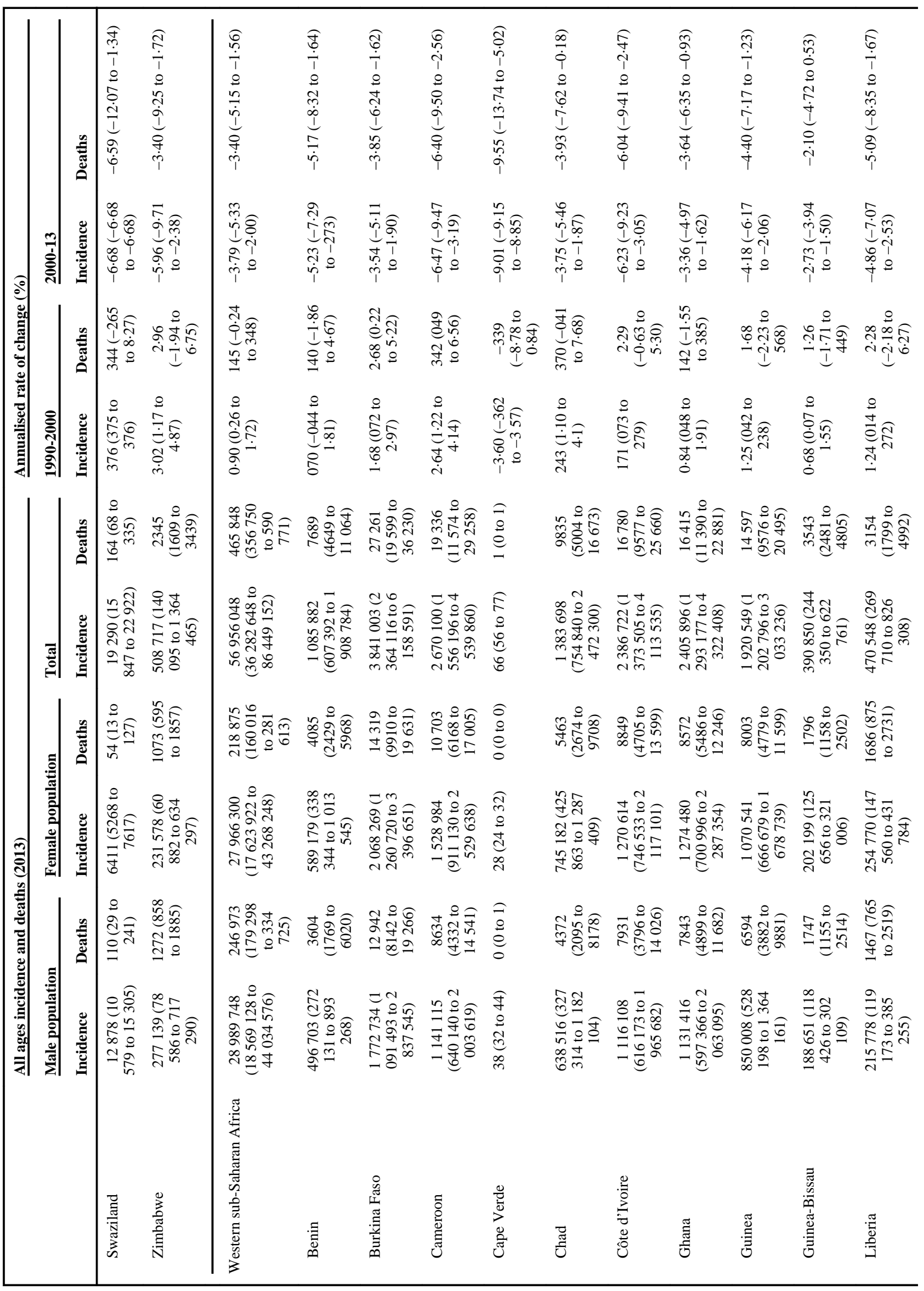




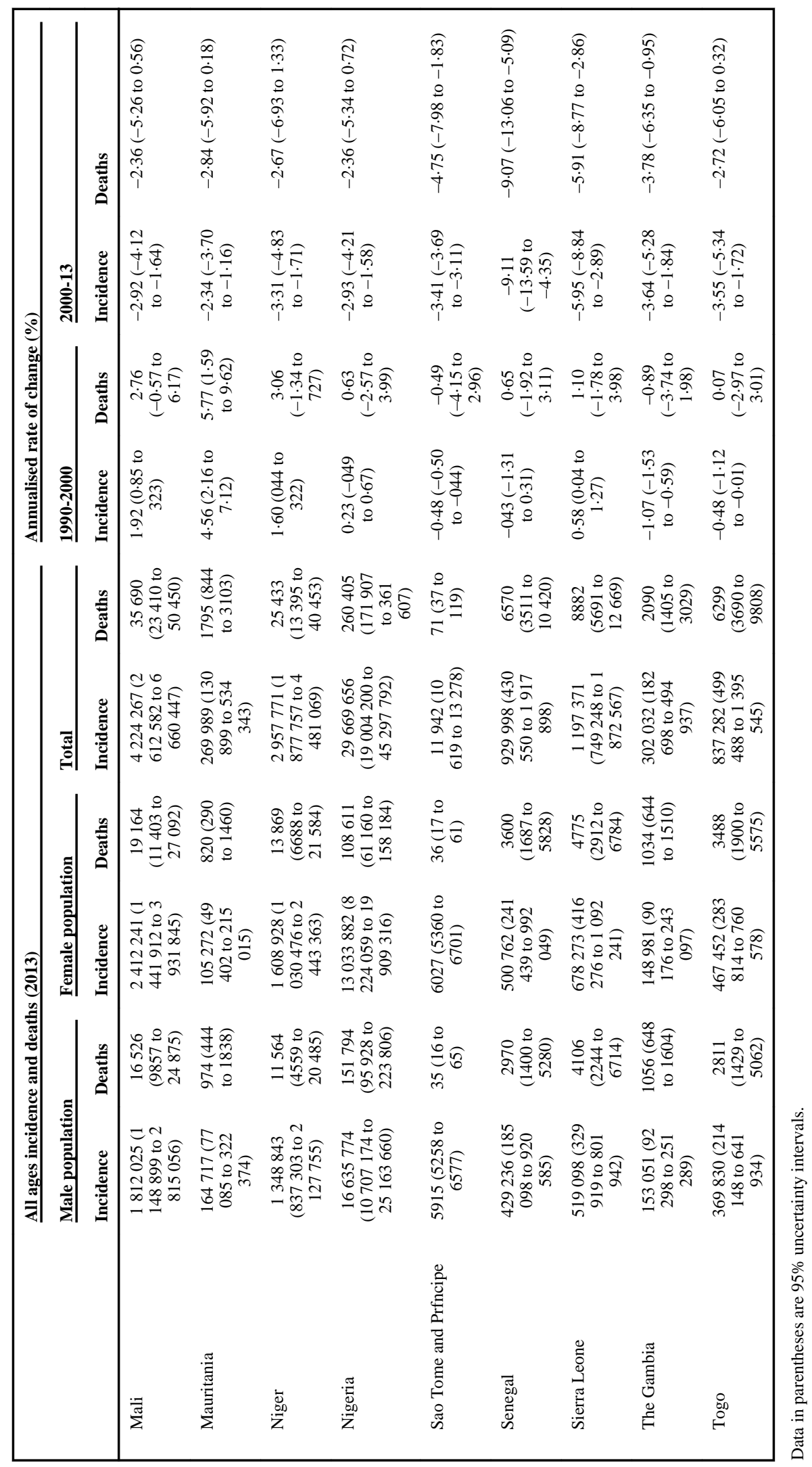


Table 8

Comparison between Global Burden of Disease 2013 verses UNAIDS 2013 HIV estimates

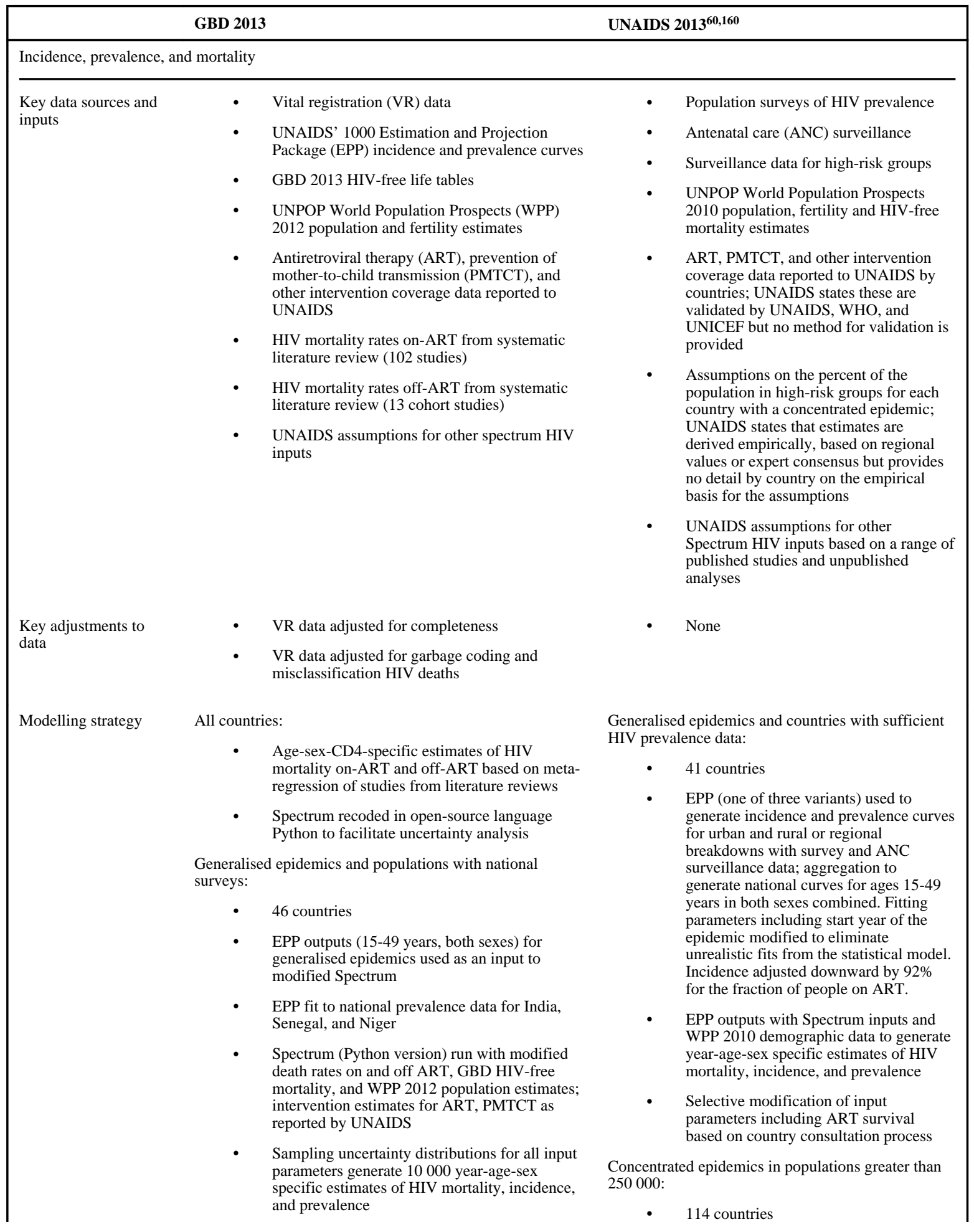


GBD 2013

- Selection of the 1000 epidemic curves that minimize the gap between GBD 2013 all-cause mortality estimates and Spectrum mortality outputs

Concentrated epidemics with VR:

- 125 countries

- $\quad$ Space-time Gaussian Process Regression (STGPR) on adjusted VR data to produce complete time series of age-sex-specific mortality

- $\quad$ EPP outputs (15-49 years, both sexes) with Spectrum inputs, GBD 2013 demographic data, and updated on-ART and off-ART mortality analysis to run Spectrum and generate 1000 year-age-sex specific estimates of HIV mortality, incidence, and prevalence

- $\quad$ Adjusted incidence from Spectrum using the ratio of ST-GPR modelled mortality to

Spectrum modelled mortality with six different assumptions of the lag from year of infection to year of death (10-15 years). This produced 6000 time series of incidence (15-49 years, both sexes)

- $\quad$ Adjusted incidence with GBD HIV-free life tables, WPP 2012 demographic data, and updated on-ART and off-ART mortality analysis to run Spectrum and generate 6000 year-age-sex specific estimates of HIV mortality, incidence, and prevalence

- $\quad$ Select 1000 with the smallest root mean squared error between model predictions of mortality and the vital registration data

Concentrated without VR:

- 17 countries

- $\quad$ Extrapolation of incidence and prevalence for countries where UNAIDS does not generate estimates by randomly selecting draws from countries in the region with estimates

- $\quad$ Regional average of all other Spectrum inputs for countries where UNAIDS does not generate estimates

- $\quad$ EPP outputs (15-49 years, both sexes) with Spectrum inputs, GBD 2013 demographic data, and updated on-ART and off-ART mortality analysis to run Spectrum and generate 1000 age-sex specific estimates of HIV mortality, incidence, and prevalence

- $\quad$ Random selection of 1000 ratios used in the incidence adjustment process from countries with relatively high prevalence

- Use selected ratios to adjust incidence in the absence of ST-GPR results, producing 1000 adjusted incidence curves (15-49 years, both sexes).

- $\quad$ Adjusted incidence with Spectrum inputs, GBD 2013 demographic data, and updated on-ART and off-ART mortality analysis to run Spectrum and generate 1000 year-age-sex specific estimates of HIV mortality, incidence, and prevalence
UNAIDS 201360,160

- $\quad$ EPP used to generate incidence and prevalence curves for high-risk groups using surveillance data for these populations

- $\quad$ Aggregation to generate national curves (15-49 years, both sexes) based on assumptions about the fraction of the population in each high-risk group

- $\quad$ EPP outputs with Spectrum progression parameters, reported ART and PMTCT coverage, and WPP 2010 demographic data to generate year-age-sex specific estimates of HIV mortality, incidence, and prevalence

- For some countries with insufficient data on prevalence in high-risk groups, reported HIV diagnoses over time and assumptions about the fraction diagnosed used

- $\quad$ Selective modification of assumptions on the percentage of the population in each high-risk group and other Spectrum input assumptions through country consultation process

Countries with populations less than 250000 :

- No estimates constructed 
GBD 2013

Uncertainty

GBD 2013 differences
- Use the 1000 EPP incidence curves consistent with the available prevalence data

- $\quad$ Generated 1000 sets of CD4 progression and CD4 specific mortality on and off ART sampled from the meta-regression of published studies

- Sample a uniform distribution of $-10 \%$ to $+10 \%$ of the mean value for all other Spectrum parameters including numbers on ART and PMTCT

- The sex ratio of incidence was sampled from a uniform distribution of $-20 \%$ to $+20 \%$ of mean value

- Major limitation is that uncertainty intervals for many parameters are sampled from an arbitrary uncertainty interval
UNAIDS $2013^{60,160}$

- $\quad$ EPP likelihood estimation of incidence reflects uncertainty in prevalence data

- Uncertainty in the percent of the population in high-risk groups or urban and rural breakdown not incorporated

- $\quad$ All uncertainty adjustments to non-EPP inputs are arbitrary and small compared with GBD

- Uncertainty propagated after point estimates generated-coefficients of variation for parameters arbitrarily selected and only for selected variables (eg, for adults ratio of fertility of HIVpositive to HIV-negative women, ratio of male to female incidence, average number of years in each CD4 category, HIV mortality without ART, HIV mortality with ART)

- $\quad$ No uncertainty incorporated for CD4 progression overtime or distribution of CD4 counts at seroconversion
- $\quad$ Recoded Spectrum in the Python programing language to enable the model to run more efficiently and allow for full uncertainty analysis

- Expanded uncertainty in Spectrum estimates of mortality, incidence, and prevalence by sampling distributions around most Spectrum inputs

- Empirically estimated uncertainty for HIV mortality on-ART and off-ART

- Used VR data when available to inform estimates of mortality for concentrated epidemics

- Identify epidemic curves and all-cause mortality estimates in countries with large epidemics that are most consistent with each other

- Sum of cause-specific mortality estimates for a country-year-age-sex group must equal allcause mortality estimate at the draw level (CoDCorrect algorithm)

N/A 
Table 9

Comparison between Global Burden of Disease 2013 verses WHO 2013 tuberculosis estimates

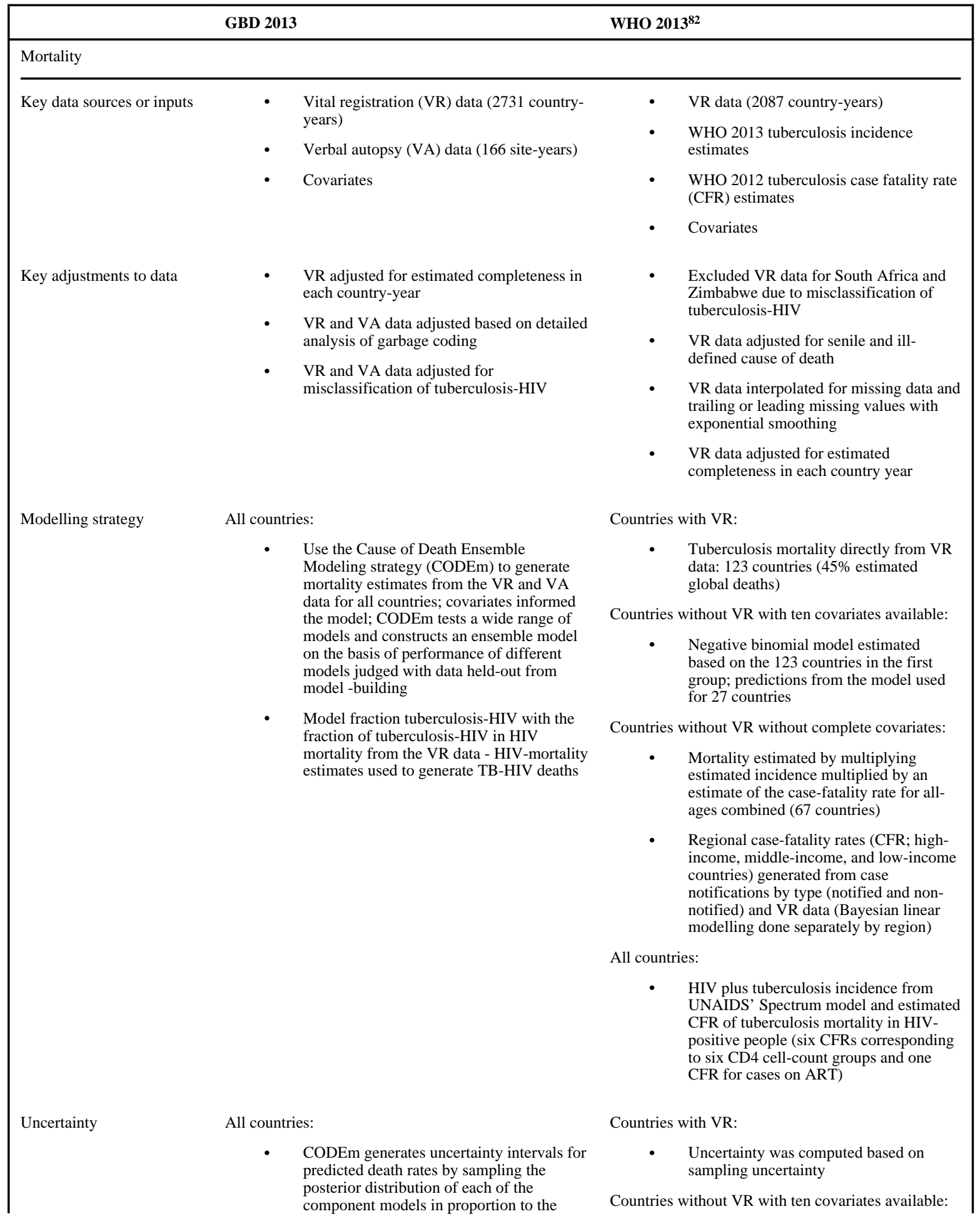




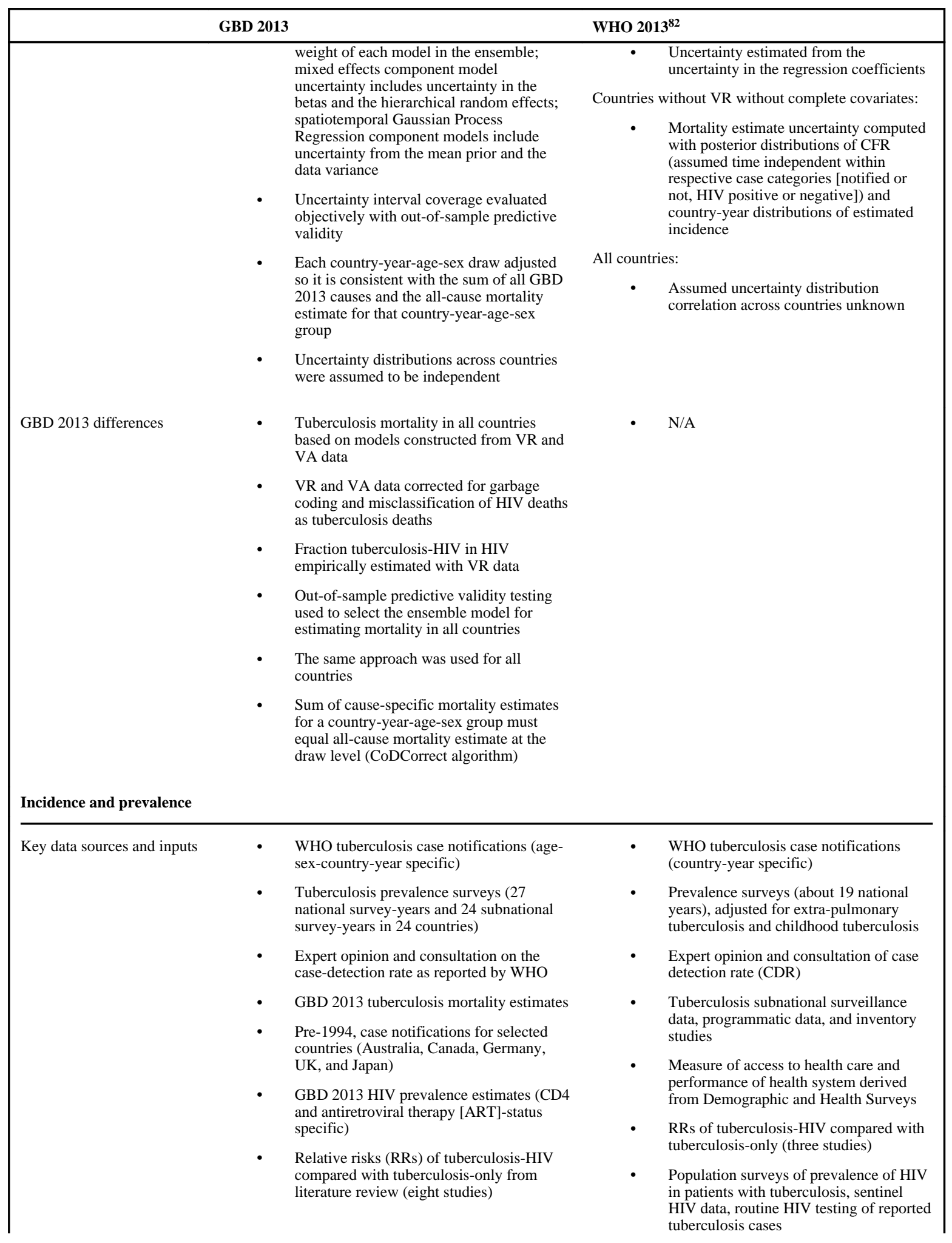




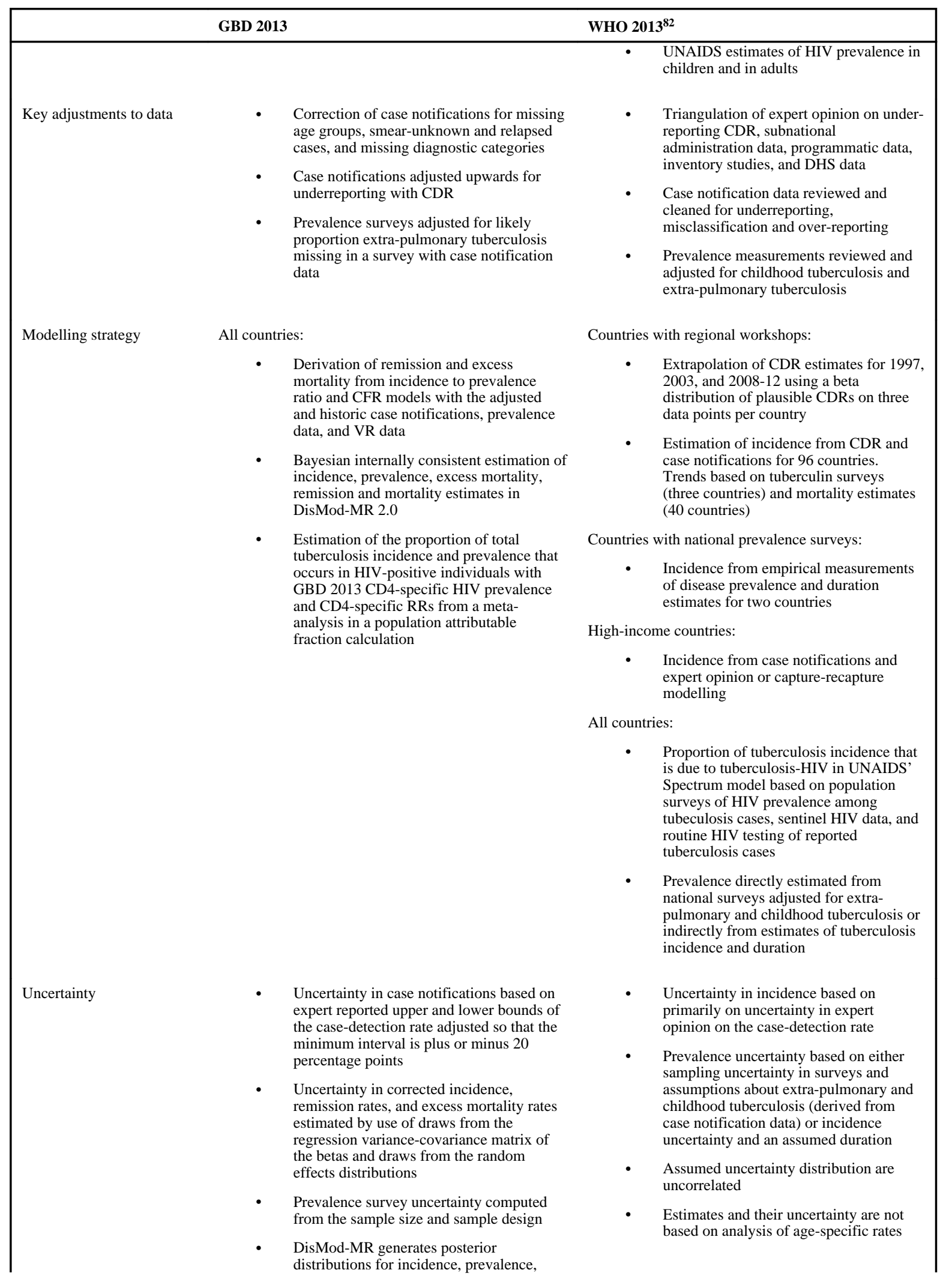




\begin{tabular}{|c|c|c|c|}
\hline & GBD 2013 & & WHO $2013^{82}$ \\
\hline GBD 2013 differences & $\begin{array}{l}. \\
. \\
. \\
. \\
\cdot\end{array}$ & $\begin{array}{l}\text { remission, and excess mortality that is a } \\
\text { function of data variance and model } \\
\text { parameter uncertainty } \\
\text { Uncertainty distributions across countries } \\
\text { assumed to be uncorrelated } \\
\text { DisMod-MR } 2.0 \text { simultaneously } \\
\text { synthesizes all available data for incidence, } \\
\text { remission, excess mortality and prevalence } \\
\text { ensuring internal consistency } \\
\text { Estimation of incidence, prevalence, } \\
\text { remission, and excess mortality is age-sex } \\
\text { specific } \\
\text { All countries modelled with the same } \\
\text { approach }\end{array}$ & - $\quad$ N/A \\
\hline
\end{tabular}


Table 10

\section{Comparison between Global Burden of Disease 2013 verses WHO 2013 malaria estimates}

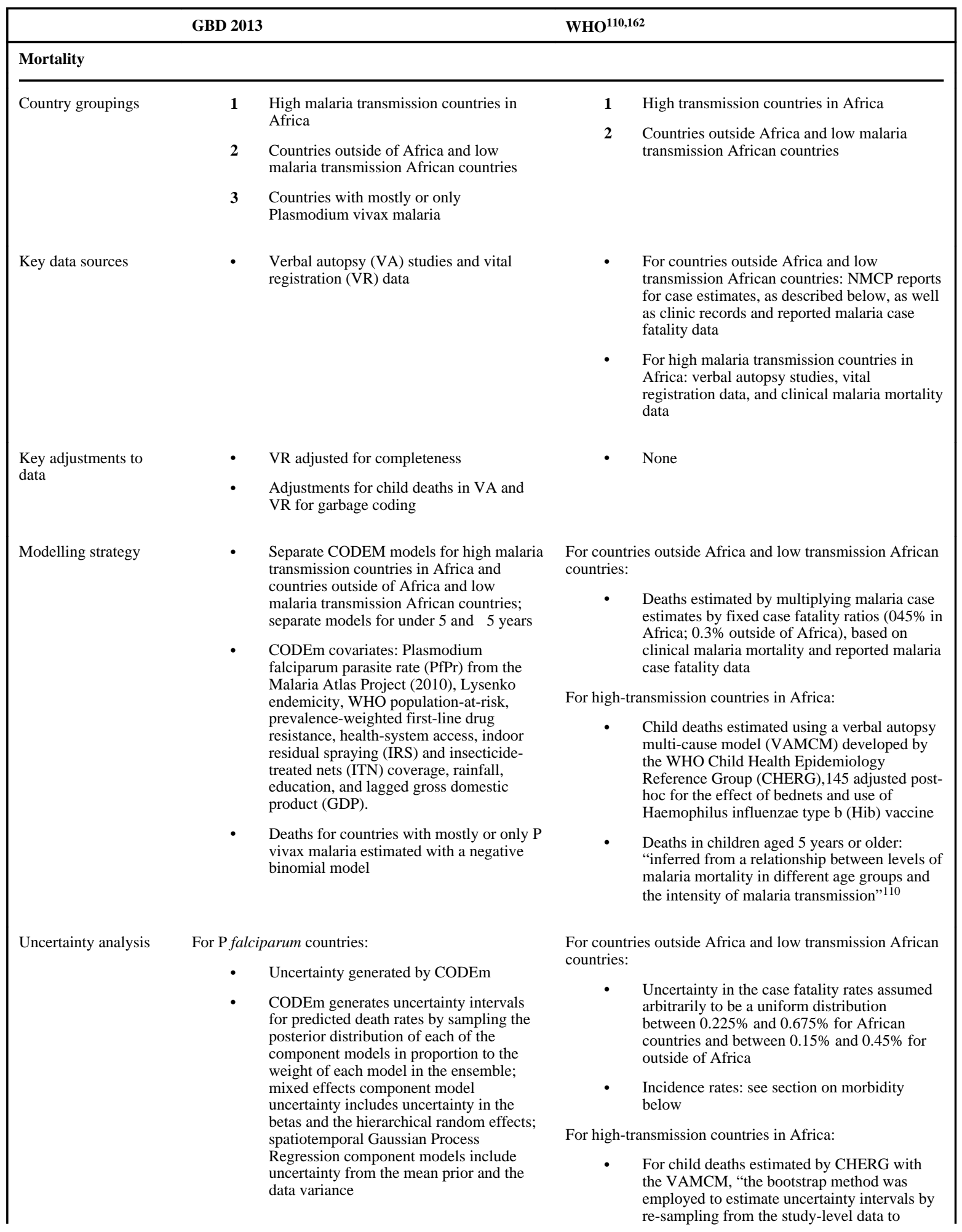




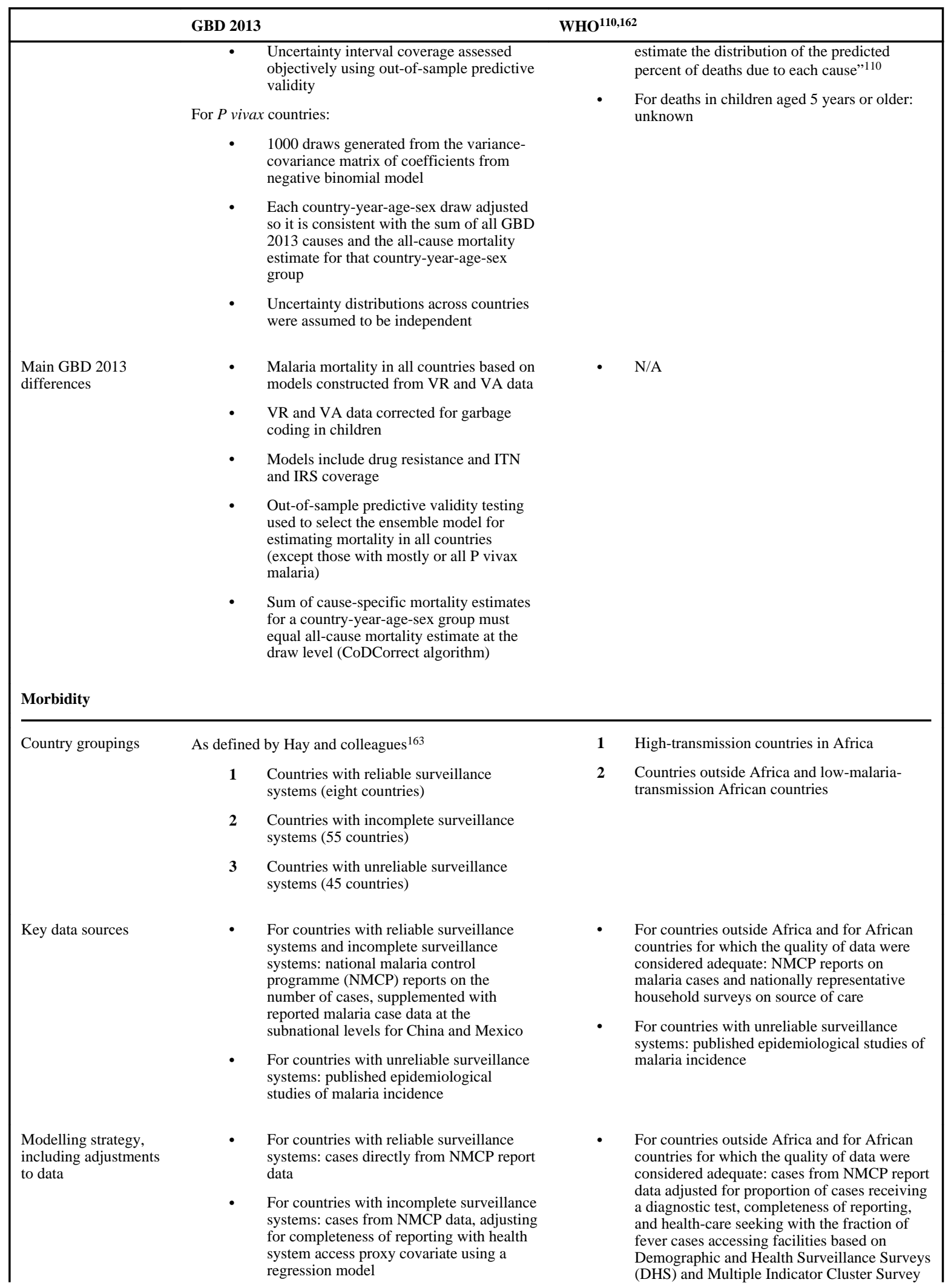




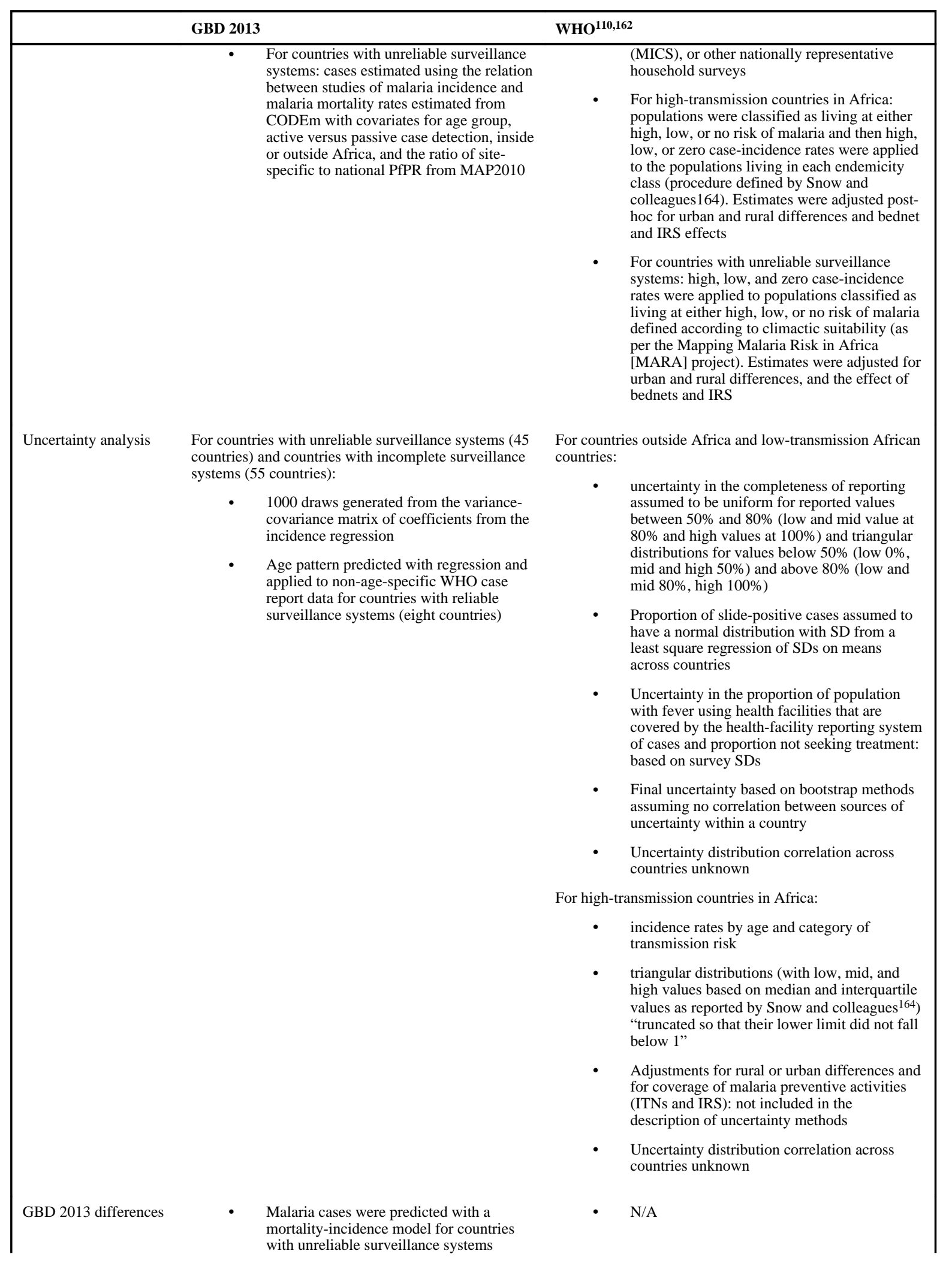




\begin{tabular}{|c|c|}
\hline GBD 2013 & WHO'10,162 $^{10}$ \\
\hline & $\begin{array}{l}\text { Predictions are adjusted for detection } \\
\text { methods (active vs passive case detection) }\end{array}$ \\
\hline
\end{tabular}

The description of WHO estimation methods was based on the World Malaria Report 2008 and World Malaria Report 2011. 